\title{
4 HANDLUNGSOPTIONEN FÜR DAS KONFLIKTMANAGEMENT IM BIOSPHÄRENPARK WIENERWALD
}

\subsection{Hinweise zum Aufbau und Gebrauch des Kapitels}

Das gegenständliche Kapitel 4 enthält Empfehlungen, Entscheidungshilfen und Anwendungshinweise für die Steuerung der in Kapitel 3 erfassten und analysierten Konfliktpotenziale zwischen den Indikator-Tierarten und Indikator-Freizeitaktivitäten auf der Ebene des Biosphärenparks. Das Kapitel enthält einem umfassenden Katalog von Maßnahmenoptionen für das Konfliktmanagement, GIS-basierte Kartendarstellungen der räumlichen Ausprägung von Konfliktpotenzialen sowie ein konkret ausgearbeitetes Konzept zur Priorisierung, Auswahl und Zuordnung von Managementalternativen zu Konfliktpotenzialsituationen und diesbezüglichen Teilräumen im Biosphärenpark. Das gesamte Kapitel ist als Maßnahmenbaukasten zu verstehen.

Die übergeordneten Ziele für das Konfliktmanagement bestehen in der vorausschauenden Vermeidung, Verringerung oder Abschwächung von Konfliktpotenzialen. Im Sinne der Konfliktprävention sollen latente Konfliktpotenziale möglichst frühzeitig erkannt und die Entstehung bzw. Eskalation von manifesten Konflikten weitest möglich vermieden werden. Wenn die Beseitigung oder Entschärfung von Konfliktpotenzialen nicht möglich ist, sollen Konfliktregelungsansätze gefunden werden, die negative Konfliktfolgen für die Erhaltung von Wildtieren und deren Lebensräumen, legitime Landnutzungsansprüche, eine nachhaltige Entwicklung in der Biosphärenparkregion sowie anderweitig bestehende öffentliche Interessen möglichst verhindern oder abschwächen.

In Kapitel 4.2 werden grundsätzlich geeignete und sinnvolle Handlungsstrategien und Maßnahmenoptionen für das Konfliktmanagement identifiziert, systematisiert und näher beschrieben. Dieses Maßnahmenportfolio ist in drei große Maßnahmengruppen gegliedert: Maßnahmen des Besuchermanagements (Freizeit- und Erholungsnutzungen); biosphärenparkweite Maßnahmen für andere Landnutzergruppen; und Maßnahmen für Monitoring und adaptives Management.

Es handelt sich um Handlungsempfehlungen aus Expertensicht, die im Rahmen des Stakeholderdialogs eingebrachte Präferenzen und Meinungen der am Projekt beteiligten Akteure weitest möglich zu berücksichtigen suchen. Das Kapitel 4.2 ist als umfassender Maßnahmenkatalog zu verstehen, aus dem - u. a. mit Hilfe der in Kapitel 4.3 vorgestellten Entscheidungshilfen - eine zielführende Auswahl getroffen werden soll. Welche Maßnahmen in welcher Kombination wann und wo zur Anwendung gelangen, hängt $u$. a. von der jeweiligen örtlichen Ausprägung des Konfliktpotenzials, der Konfliktstruktur, den Managementzielen sowie den Präferenzen der zuständigen Akteure im Biosphärenpark ab. In jedem Fall ist eine differenzierte Beurteilung der örtlichen Gegebenheiten und Rahmenbedingungen notwendig. $\mathrm{Zu}$ beachten ist weiters in jedem Fall die biosphärenparkweite und sektorübergreifende $\mathrm{Ab}$ stimmung von einzelnen präferierten Maßnahmenpaketen, denn diese können stets nur als kohärentes Gesamtpaket die gewünschte Wirksamkeit entfalten.

Kapitel 4.3 gibt Entscheidungshilfen für die Auswahl und Kombination von Maßnahmenalternativen in Abhängigkeit von Ausprägungen des Konfliktpotenzials. Ausgehend von den biosphärenparkweit vorkommenden Indikator-Tierarten wird eine Verknüpfung der Managementoptionen aus Kapitel 4.2 mit unterschiedlichen Konfliktpotenzialen vorgenommen. Hierzu werden in Kapitel 4.3 generelle Ziele des Konfliktmanagements im Biosphärenpark in Bezug auf die einzelnen Indikatorarten formuliert, spezifische Zielsetzungen für unterschiedliche Konfliktpotenzialsituationen und -räume abgeleitet sowie Managementoptionen (aus 
dem umfassenden Maßnahmenkatalog in Kapitel 4.2), die für bestimmte Konfliktsituationen prioritär geeignet erscheinen, in Übersichtsmatritzen den jeweiligen Konfliktpotenzialausprägungen zugeordnet. Weiters werden für jede Indikatorart spezifische sektorale Maßnahmen für die Landnutzergruppen Land- und Forstwirtschaft, Jagd, Naturschutz und Raumordnung formuliert und wiederum in Übersichtsmatritzen deren vorrangige Eignung für unterschiedliche Konfliktpotenzialsituationen eingeschätzt. GIS-basierte Kartendarstellungen sollen für jede der Indikatorarten die räumliche Zuordnung von prioritären Maßnahmen zu Teilräumen im Biosphärenpark erleichtern.

In Kapitel 4.4 wird am Beispiel der Indikatorart Ziesel und des Intensivuntersuchungsgebietes Perchtoldsdorfer Heide ein „good practice“-Beispiel für Konfliktaustragung und Konfliktbewältigung präsentiert. 


\subsection{Maßnahmenoptionen für das Konfliktmanagement}

Die nachstehenden Empfehlungen für das Konfliktmanagement umfassen Handlungsstrategien und Maßnahmenoptionen, die für das Management der auf biosphärenparkweiter Maßstabsebene ermittelten Konfliktpotenziale (siehe Kap. 3.4) grundsätzlich geeignet und sinnvoll sind.

Der Maßnahmenkatalog ist in Maßnahmengruppen gegliedert, stellt ein umfassendes Portfolio von Managementoptionen dar und ist als "Maßnahmenbaukasten“ zu verstehen. Dies bedeutet, dass die Anwendung von Maßnahmenoptionen in unterschiedlichen Kombinationen möglich und zielführend ist. Viele der Maßnahmenoptionen stehen untereinander in Zusammenhang; auf wechselseitige Abhängigkeiten zwischen Maßnahmen wird in der Beschreibung teils hingewiesen. Manche Maßnahmen sollten grundsätzlich nur in Verbindung miteinander angewendet werden; so sollte beispielsweise die Vereinbarung von räumlichzeitlichen Nutzungsrestriktionen für Erholungssuchende stets im Rahmen kooperativer Konfliktlösungsstrategien erfolgen. Die in Kap. 4.2.1.2 empfohlenen querschnittsorientierten Maßnahmen sind grundsätzlich von biosphärenparkweiter Relevanz, d.h. sie haben nicht a priori einen konkreten räumlichen Bezug.

Wo angebracht und notwendig, wird bei einzelnen Maßnahmengruppen auf Voraussetzungen, Begleitmaßnahmen, mögliche Risiken und Umsetzungshindernisse gesondert hingewiesen.

Weitere Maßnahmenvorschläge, die an sektorale Landnutzergruppen (Land- und Forstwirtschaft, Jagdwirtschaft, Naturschutz, Raumordnung) adressiert sind, sind in Kapitel 4.3 für jede der Indikator-Tierarten zusammengestellt.

Ein methodischer Ansatz zur Auswahl und Priorisierung von Maßnahmenoptionen aus dem Gesamt-Maßnahmenportfolio ist in Kapitel 4.3 ausgearbeitet. Ausgehend von den einzelnen Indikator-Tierarten erfolgt dort über Konfliktpotenzialkarten und Verknüpfungsmatritzen die Zuordnung von einzelnen Maßnahmenoptionen zu unterschiedlichen Konfliktpotenzialausprägungen und diesbezüglichen Konflikträumen im Biosphärenpark.

Nachstehende Tabelle 38 gibt einen Überblick über die Struktur der Maßnahmenoptionen. Die Kennziffern der Maßnahmenpakete und -optionen (z. B. A1) sollen das Auffinden von Maßnahmen in den Übersichtstabellen in Kap. 4.3 erleichtern. 
Tabelle 38: Überblick über die Struktur der Maßnahmenempfehlungen

\begin{tabular}{|c|c|c|c|c|}
\hline Kategorie & Maßnahmengruppe & \multicolumn{2}{|c|}{ Maßnahmenpaket } & \multirow{2}{*}{\begin{tabular}{|l}
$\begin{array}{l}\text { Maßnahmen- } \\
\text { optionen }\end{array}$ \\
A1-A5
\end{tabular}} \\
\hline \multirow{10}{*}{$\begin{array}{l}\text { 4.2.1 Besucher- } \\
\text { management - } \\
\text { Freizeit- und Erho- } \\
\text { lungsnutzungen }\end{array}$} & \multirow{5}{*}{$\begin{array}{l}\text { 4.2.1.1 Kleinräumige } \\
\text { Maßnahmenoptionen }\end{array}$} & A) & $\begin{array}{l}\text { Räumlich-zeitliche Nutzungsrestriktionen in sensiblen Gebieten mit } \\
\text { erhöhtem Konfliktpotenzial }\end{array}$ & \\
\hline & & B) & Besucherlenkung durch De-Attraktivierung von sensiblen Gebieten & $\mathrm{B} 1-\mathrm{B} 3$ \\
\hline & & C) & $\begin{array}{l}\text { Schaffung von kleinräumigen Kompensations- bzw. Alternativange- } \\
\text { boten zur Besucherlenkung }\end{array}$ & $\mathrm{C} 1-\mathrm{C} 8$ \\
\hline & & D) & $\begin{array}{l}\text { Verstärkte Information, Kontrolle und Vollzug in Schwerpunktberei- } \\
\text { chen }\end{array}$ & $\mathrm{D} 1-\mathrm{D} 3$ \\
\hline & & E) & $\begin{array}{l}\text { Teilraumbezogene Beurteilung des Managementbedarfs (bei unkla- } \\
\text { rem Konfliktpotenzial) }\end{array}$ & E1 \\
\hline & \multirow{5}{*}{$\begin{array}{l}\text { 4.2.1.2 Querschnitts- } \\
\text { orientierte Handlungs- } \\
\text { optionen und Begleit- } \\
\text { maßnahmen }\end{array}$} & F) & Information, Kommunikation, Öffentlichkeitsarbeit & F1-F12 \\
\hline & & G) & Kooperative Konfliktlösungsstrategien & $\mathrm{G} 1-\mathrm{G} 4$ \\
\hline & & $\mathrm{H})$ & Kontrolle und Vollzug von Verhaltensregeln & $\mathrm{H} 1-\mathrm{H} 2$ \\
\hline & & I) & Biosphärenparkweite Verhaltensregeln & 11 \\
\hline & & J) & $\begin{array}{l}\text { Auswahl und Ausgestaltung von Querschnittsmaßnahmen in Abhän- } \\
\text { gigkeit von Einzelfallprüfungen des Managementbedarfs (bei unkla- } \\
\text { rem Konfliktpotenzial) }\end{array}$ & $\mathrm{J} 1$ \\
\hline \multirow{2}{*}{\multicolumn{2}{|c|}{$\begin{array}{l}\text { 4.2.2 Generelle Maßnahmenoptionen für andere } \\
\text { Landnutzergruppen }\end{array}$}} & K) & Raumplanung, Infrastrukturplanung & $\mathrm{K} 1-\mathrm{K} 6$ \\
\hline & & L) & Sektorübergreifende Kooperation aller Landnutzungsbereiche & $\mathrm{L} 1-\mathrm{L} 3$ \\
\hline \multirow{3}{*}{\multicolumn{2}{|c|}{ 4.2.3 Monitoring und adaptives Management }} & M) & Besuchermonitoring & $\mathrm{M} 1-\mathrm{M} 3$ \\
\hline & & N) & Erfolgskontrolle zu vereinbarten Nutzungsregeln & N1 \\
\hline & & O) & Monitoring von Wildtierpopulationen und -lebensräumen & $01-08$ \\
\hline \multirow{4}{*}{$\begin{array}{l}\text { 4.3 Spezifische Maß- } \\
\text { nahmenoptionen für } \\
\text { weitere Landnutzer- } \\
\text { gruppen in Bezug auf } \\
\text { die Indikatorarten }\end{array}$} & 4.3.2.1.6 Schwarzstorch & \multicolumn{3}{|c|}{ Naturschutz, Forstwirtschaft, Landwirtschaft, Jagdwirtschaft } \\
\hline & 4.3.2.2.6 Rothirsch & \multicolumn{3}{|c|}{ Forstwirtschaft, Landwirtschaft, Jagdwirtschaft, Raumordnung } \\
\hline & \begin{tabular}{|l|} 
4.3.2.3.6 Wildschwein \\
\end{tabular} & \multicolumn{3}{|c|}{ Forstwirtschaft, Landwirtschaft, Jagdwirtschaft, } \\
\hline & 4.3.2.4.6 Auerhuhn & \multicolumn{3}{|c|}{ Naturschutz, Forstwirtschaft, Jagdwirtschaft, Raumordnung } \\
\hline
\end{tabular}




\subsubsection{Besuchermanagement - Freizeit- und Erholungsnutzungen}

\subsubsection{Kleinräumige Maßnahmenoptionen}

\section{A) Räumlich-zeitliche Nutzungsrestriktionen in sensiblen Gebieten mit erhöhtem Konfliktpotenzial}

Ziele:

- Sicherung und Erhaltung derzeit störungsarmer, ökologisch sensibler Gebiete durch Geringhalten der Nachfrage nach Erholungsaktivitäten

- Verringerung des Konfliktpotenzials durch gezielte Nutzungsberuhigung in ökologisch sensiblen Gebieten mit derzeit erhöhter Nutzungsintensität

\section{Handlungsstrategie:}

- Rechtliche Nutzungsverbote und -gebote zur Einschränkung der räumlichen Nutzung bzw. der zeitlichen Nutzungsstruktur

- Freiwillige Vereinbarungen zur Einschränkung der räumlichen Nutzung bzw. der zeitlichen Nutzungsstruktur

\section{Maßnahmenoptionen:}

A1) Vereinheitlichung der rechtlich verankerten Nutzungsverbote und -gebote: Die Kernzonen wurden im niederösterreichischen Teil des Biosphärenparks als Naturschutzgebiete verordnet, im Wiener Teil hingegen als Landschaftsschutzgebiete. Hieraus ergeben sich für Kernzonen in Wien und in Niederösterreich unterschiedliche rechtliche Bestimmungen betreffend Nutzungsverbote und -gebote. Das niederösterreichische Naturschutzgesetz sieht innerhalb von Naturschutzgebieten ein Betretungsverbot außerhalb von in der Verordnung bezeichneten Wegen und Bereichen vor (NÖ NSG 2000 idgF. §11), während in Landschaftsschutzgebieten nach dem Wiener Naturschutzgesetz kein Wegegebot für Freizeitnutzer besteht (Wiener Naturschutzgesetz idgF., §22). Hiermit fehlt im Wiener Teil des Biosphärenparks eine wesentliche rechtliche Handhabe, um wirksame Maßnahmen zur Besucherlenkung in Kernzonen verbindlich umsetzen zu können. Darüber hinaus sind unterschiedlich strenge Bestimmungen für die Nutzung in Kernzonen in den beiden Teilen des Biosphärenparks gegenüber den Freizeitnutzern schwer kommunizierbar und in der Praxis wenig umsetzungsfähig. Die rechtliche Unterschutzstellung der Kernzonen sowie die damit verbundenen Nutzungsgebote und verbote sollten daher in beiden Bundesländern möglichst angeglichen werden, wobei ein generelles Wegegebot nach dem niederösterreichischen Muster anzustreben wäre. Neben einer gleichwertigen naturschutzrechtlichen Ausweisung könnte dieses Ziel auch durch die Angleichung der Biosphärenparkgesetze in beiden Bundesländern erreicht werden: Nach dem niederösterreichischen Biosphärenparkgesetz kann der Schutz der Kernzonen insbesondere durch die Erklärung zum Naturschutzgebiet „oder durch vertragliche Maßnahmen, die einen gleichwertigen Schutz gewährleisten, erfolgen." Eine ähnliche Bestimmung im Wiener Biosphärenparkgesetz würde es grundsätzlich erlauben, die Bestimmungen für alle Kernzonen des Biosphärenparks länderunabhängig und somit einheitlich zu gestalten. 
A2) Verzicht auf Ausbau der Wegeinfrastruktur in Kernzonen und anderen ökologisch sensiblen Räumen: In den Kernzonen sowie in Räumen mit hoher ökologischer Sensibilität (z. B. Lebensraum des Schwarzstorchs, Rotwild-Kerngebiete, potenzielle Auerhuhn-Habitate, etc.) bzw. hohem Konfliktpotenzial zwischen Lebensraumfunktionen und Freizeitaktivitäten sollte generell der Imperativ des Verzichts auf die Planung und Errichtung neuer Wegeinfrastruktur zur Anwendung kommen. Dies gilt insbesondere auch für Wege, die zu forst- oder jagdwirtschaftlichen Zwecken errichtet werden, da diese im Regelfall eine erhöhte Intensität von Freizeitfolgenutzungen induzieren.

A3) Kernzonengerechtes Wegenetz (Verlegung bzw. Auflassung von Wegen): Das Wegenetz stellt grundsätzlich eine effektive Möglichkeit zur räumlichen Lenkung von Besucherströmen dar. Insbesondere zur Erreichung von Zielen des Prozessnaturschutzes in den Kernzonen sollte neben der Durchsetzung des Wegegebots die Freizeit- und Erholungsnutzung vermehrt über die gezielte Gestaltung des Wegeangebots gesteuert werden. Zu diesem Zweck sollten Instrumente wie die Umlegung von bestehenden Wegen in Bereiche außerhalb von Kernzonen bzw. die Auflassung von Wegen verstärkt geprüft und angewendet werden, wenn es zur Konfliktminimierung und für die Erfüllung einer Funktion von Kernzonen als Ruheund Rückzugszone für Wildtierarten erforderlich ist. Derartige Lösungsansätze bedürfen in jedem Fall der vorangehenden partizipativen und konsensorientierten Aushandlung mit den Vertretern der betroffenen Nutzergruppen (wie Alpenverein, Tourismusklub, etc.). Hierbei sind Wegetraditionen besonders zu berücksichtigen, d.h. bei Änderungen von seit langem bestehenden und von z. B. Alpinvereinen erhaltenen Wegen ist besondere Sensibilität erforderlich. Die kernzonengerechte Gestaltung des Wegenetzes ist in den Managementplänen von Kernzonen zu verankern.

A4) Freiwillige Vereinbarungen zu örtlich begrenzten, räumlichen und/oder zeitlichen Nutzungseinschränkungen: Räumlich und/oder zeitlich begrenzte Wegegebote, Betretungsverbote oder anderweitige Nutzungseinschränkungen, die über bestehende, z. B. forstrechtlich verankerte Bestimmungen hinausgehen, können grundsätzlich auch außerhalb von Kernzonen in anderweitig ökologisch sensiblen oder konfliktintensiven Räumen angebracht und zielführend sein. Im Regelfall werden hierfür jedoch freiwillige Vereinbarungen zwischen Grundeigentümern und relevanten Nutzergruppen im Rahmen partizipativer Aushandlungsprozesse erforderlich sein (vgl. Maßnahmengruppe G1 - G4). Eine spezifische Anwendungsmöglichkeit können beispielsweise Vereinbarungen zu definierten störungsfreien Jagdzeiten (z. B. Montag morgens) bzw. Jagdtagen zum Zwecke der effizienten Abschusserfüllung bilden (v.a. zur Regulation des Schwarzwilds). Zu berücksichtigen ist hierbei, dass örtlich oder zeitlich begrenzte Sonderregelungen besondere Herausforderungen hinsichtlich Kommunizierbarkeit, Akzeptanz, Befolgung und Kontrolle verursachen.

A5) Jagdrechtlich begründete Nutzungsrestriktionen (Wildschutzgebiete, befristete jagdliche Sperrgebiete): Das Niederösterreichische Jagdgesetz (NÖ Jagdgesetz 1974 idgF.) sieht die Möglichkeit der befristeten oder unbefristeten Einrichtung von Wildschutzgebieten vor (§ 94a). Als Wildschutzgebiete bewilligt werden dürfen Flächen, die als besonders bevorzugte Einstandsgebiete Ruhezonen für das Wild sind oder solche, die zum Brüten oder Setzen bevorzugt angenommen werden; Wildschutzgebiete dienen der Verhinderung einer Beunruhigung des Wildes und daraus entstehender Wildschäden. Die Erklärung eines Gebietes zum Wildschutzgebiet hat zur Wirkung, dass jagdfremde Personen das betreffende 
Gebiet abseits von öffentlichen Wegen und Straßen nicht betreten dürfen. Dieses jagdrechtliche Instrument bietet somit eine grundsätzliche Handlungsoption, um in wildbiologisch begründeten Situationen eine Nutzungsberuhigung von Ruhe- und Rückzugsbereichen für dem Jagdrecht unterliegende Wildtiere (z.B. Rotwildkerngebiete, Auerhuhn-Quellgebiete im Falle einer gewünschten Wiederansiedlung) durchzusetzen. Eine analoge Wirkung des Betretungsverbotes bzw. Wegegebotes für jagdfremde Personen geht vom Instrument der Sperre von Jagdgebieten aus (§ 94); dieses ist im niederösterreichischen Jagdgesetz insbesondere zum Zwecke der Durchführung von Treibjagden vorgesehen. Insbesondere in Situationen, in denen die effektive Durchführung des Regulationsabschusses bzw. die Schwerpunktbejagung in Wildschadensgebieten von Rotwild und Schwarzwild im öffentlichen Interesse ansonsten durch intensive Erholungsnutzungen nicht gewährleistet werden kann, bietet dieses Instrument eine Ansatzmöglichkeit zur räumlich begrenzten und zeitlich befristeten Vermeidung von Konflikten zwischen Jagdbetrieb und Freizeitnutzungen sowie daraus resultierenden Sicherheitsrisiken für Besucher. Darüber hinaus gilt der Bereich im Umkreis von 200 Metern um Wildfütterungen während der Fütterungsperiode ex lege als für jagdfremde Personen gesperrt. Sowohl für Wildschutzgebiete als auch für jagdliche Sperrgebiete ist eine Kundmachung durch geeignete Hinweise (Beschilderung) vorgeschrieben. Grundsätzlich sollten jagdrechtlich verankerte Instrumente mit der Wirkung von Nutzungsrestriktionen für Erholungssuchende nur zurückhaltend und in begründeten Ausnahmesituationen zur Nutzungsberuhigung eingesetzt werden; als genereller Ansatz zum Besuchermanagement sind sie nicht geeignet. Es wird empfohlen, die Anwendung beider Instrumente vorher mit den von den Restriktionen betroffenen Nutzergruppen abzustimmen. Es ist unbedingt darauf zu achten, dass Beschilderungen entsprechend der zeitlichen Befristung von Sperr- und Wildschutzgebieten auch fristgerecht wieder entfernt werden.

\section{B) Besucherlenkung durch De-Attraktivierung von sensiblen Gebieten}

Ziel:

- Nutzungsberuhigung von ökologisch sensiblen bzw. konfliktträchtigen Gebieten

Handlungsstrategie:

- Verringerung der Attraktivität und Zugänglichkeit von sensiblen Gebieten für Freizeitund Erholungsnutzer durch Lenkungsmaßnahmen

\section{Maßnahmenoptionen:}

B1) Landschaftsgestalterische Maßnahmen: Vergleichsweise „sanfte“ Lenkungsmaßnahmen zur „De-Attraktivierung“ von ökologisch sensiblen Räumen mit erhöhtem Konfliktpotenzial können landschaftsgestalterische bzw. vegetationstechnische Maßnahmen umfassen, die zu einer Verringerung der Zugänglichkeit für Erholungssuchende führen. So können beispielsweise unerlaubte bzw. unerwünschte Trampelpfade und informelle Wege durch Bepflanzung mit Dornen- oder Stachel bewehrten Sträuchern oder durch gezielte Verlegung mit liegenden Baumstämmen unattraktiv gemacht werden. Derartige, gering aufwandsintensive Maßnahmen können auch eine Wegesperrung unterstützen. Ein weiteres Beispiel bildet die gezielte Erhaltung bzw. Pflanzung von gewässerbegleitenden Ufergehöl- 
zen, die gegenüber Nahrungshabitaten des Schwarzstorchs Sicht- und Lärmschutz bieten und die Zugänglichkeit für Besucher verringern.

B2) Verzicht auf Schneeräumung: Durch den Verzicht auf Schneeräumung von Wegen und von Zufahrten bzw. Zustiegen zu z. B. Wildfütterungen kann die Zugänglichkeit zu wildökologisch sensiblen Bereichen im Winter gezielt reduziert und hierdurch eine Störung von Wildtieren und Jagdbetrieb vermindert werden. Im unmittelbaren Wohnumfeld und bei intensiverer Naherholungstätigkeit ist die Wirksamkeit dieser Maßnahme allerdings eingeschränkt; in diesem Fall sollte die Jagd durch geeignetere Standortwahl von jagdbetrieblichen Einrichtungen die bestehende Erholungsnachfrage berücksichtigen.

B3) Management von Infrastruktur mit Schleusenfunktion: Infrastruktureinrichtungen, die als Ausgangspunkte und Einstiegspforten für Freizeitaktivitäten in ökologisch sensiblen Bereichen fungieren, wie z. B. Parkplätze, können in begründeten Fällen aufgelassen, in ihrer Kapazität reduziert (z. B. Zahl von Parkplätzen) oder durch Einhebung von Gebühren (z. B. Parkgebühr) de-attraktiviert werden. In vielen Fällen ist eine zeitliche Begrenzung derartiger Maßnahmen sinnvoll, z. B. auf den Winter in Bezug auf Winterlebensräume des Rotwilds bzw. auf den Sommer in Bezug auf Bruthabitate des Schwarzstorchs.

\section{C) Schaffung von kleinräumigen Kompensations- bzw. Alternativangeboten zur Be- sucherlenkung}

Ziele:

- Sicherung und Erhaltung derzeit störungsarmer, ökologisch sensibler Gebiete durch Umlenkung von Besucherströmen

- Verringerung der Störungsintensität durch Freizeit- und Erholungsaktivitäten in derzeit intensiv genutzten ökologisch sensiblen Gebieten mit hohem Konfliktpotenzial durch kleinräumige Attraktivierung in weniger sensiblen Gebieten

- Verbesserung der Kommunizierbarkeit und Akzeptanz von Nutzungsrestriktionen in der Fläche bzw. in Gebieten mit hohem Konfliktpotenzial durch Schaffung „legalisierter" Kompensationsangebote

Handlungsstrategie:

- Kleinräumige Attraktivierung von ökologisch wenig sensiblen Gebieten mit entsprechender Standorteignung

- Umlenkung von Besuchern in ökologisch wenig sensible Gebiete und Funktionstrennung durch Neuschaffung von Kompensations- bzw. Alternativangeboten für Freizeitnutzer

- Kanalisierung von nicht erlaubten Verhaltensweisen durch punktuelle Schaffung legaler Freizeitangebote

Maßnahmenoptionen:

C1) Hundeauslaufbereiche: Anlage von attraktiv gestalteten, eingezäunten und wildfreien Hundefreilaufzonen („Hundewälder“, „Gassistrecken“) in Siedlungsnähe. Diese Hundeauslaufbereiche sollen sowohl tagsüber als auch nachts benutzbar sein und sollen insbesondere Erholungsbedürfnisse der täglichen 
Wohnumfeldnutzung befriedigen. Derartige Modelle haben sich beispielsweise in Deutschland und Dänemark zur Verringerung des Konfliktpotenzials durch Erholungsaktivitäten mit Hunden bewährt.

C2) Night Trails für Jogger: Freigabe von einzelnen, weggebundenen Laufstrecken auch zur Nachtzeit (nach Einbruch der Abenddämmerung und vor Sonnenaufgang) im Tageserholungsbereich in ökologisch wenig sensiblen Gebieten. Wesentlich hierbei sind die vorherige Prüfung des örtlichen Bedarfs und der Streckeneignung (ökologische, jagdliche und raumstrukturelle Verträglichkeit) sowie die Abstimmung unter allen betroffenen Stakeholdern und Nutzergruppen (Laufvereine, Gemeinden, Grundeigentümer, Jagd, etc.).

C3) Night Trails und Winter Trails für Mountainbiker: Ausweitung der zulässigen Benützungszeiten von einzelnen bestehenden, ausgewiesenen MountainbikeStrecken auch in den späten Abend- und frühen Morgenstunden (nach Abenddämmerung und vor Sonnenaufgang) im Tageserholungsbereich der Wohnumfeldnutzung und in ökologisch wenig sensiblen Gebieten. Für möglichst dieselben Strecken sollte auch eine Lockerung bzw. Aufhebung des Winterfahrverbotes für Mountainbiker (November bis Februar) einschließlich der Schneeräumung im Winter geprüft werden. Die selektive Freigabe der Benützung zur Nachtzeit sowie im Winter ist auf Bestandsstrecken zu beschränken, d.h. es soll zu keiner Errichtung neuer Wegeinfrastruktur kommen. Wesentlich hierbei sind die bedarfsorientierte Streckenauswahl, die sorgfältige vorangehende Prüfung der Streckeneignung nach Kriterien der ökologischen, jagdlichen und raumstrukturellen (z. B. Erreichbarkeit ohne privates Kfz) Verträglichkeit sowie die Abstimmung unter allen betroffenen Stakeholdern und Nutzergruppen (Grundeigentümer, Gemeinden, Jagd, Mountainbike-Vertreter, etc.). Idealerweise sollte die Planung von für den Nacht- und Winterbetrieb freizugebenden Strecken in ein biosphärenparkweit abgestimmtes Mountainbike-Routenkonzept eingebettet sein.

C4) Mountainbike Technik Trails: Gezielte Anlage von einzelnen, attraktiv gestalteten Technik-Parcours für Mountainbiker (mit Sprungschanzen etc.). Zur Vermeidung von zusätzlicher Flächeninanspruchnahme im Naturraum durch die erforderlichen baulichen Maßnahmen, um keinen zusätzlichen motorisierten Individualverkehr zu erzeugen, und um die grundsätzliche Biosphärenparkverträglichkeit zu gewährleisten, sollten derartige Anlagen vorzugsweise im unmittelbaren Siedlungsnahbereich, innerhalb des bestehenden Siedlungsgebietes bzw. außerhalb der Grenzen des Biosphärenparks errichtet werden. Auch hier wäre die Einbettung in ein biosphärenparkweites Mountainbike-Routenkonzept anzustreben.

C5) Mountainbike Downhill-Strecken: Durch die selektive Ausgestaltung einzelner geeigneter Streckenteile des bestehenden ausgewiesenen MountainbikeStreckennetzes als Downhill-Strecken bzw. durch die Neuanlage geeigneter Strecken könnten bestehende illegale Mountainbike-Downhill-Aktivitäten in der Fläche (einschließlich illegaler Baumaßnahmen) legal und kleinräumig begrenzt kanalisiert werden. Auch hier ist eine sorgfältige Prüfung der Streckenauswahl und der Streckeneignung nach ökologischen, raumstrukturellen (Verkehrsanbindung, Erreichbarkeit mit dem Rad, etc.), jagdwirtschaftlichen und bedarfsorientierten Kriterien unerlässlich. Die Einbettung in ein biosphärenparkweites MountainbikeRoutenkonzept ist anzustreben.

c6) Betreute Picknickplätze: Die bedarfsorientierte Ausweisung und Anlage geeigneter, betreuter und entsprechend ausgestatteter Picknick- und Lagerplätze im un- 
mittelbaren Siedlungsnahbereich kann zur Kanalisierung diesbezüglicher Aktivitäten beitragen. Flächige Störungen von Äsungsflächen, von zur jagdlichen Regulation von Rot- und Schwarzwild benötigten Schussfeldern sowie u. U. von Nahrungshabitaten des Schwarzstorchs können durch diese Lenkungsmaßnahme vermindert werden. Neben ökologischen bzw. naturräumlichen Eignungskriterien sind bei der Standortwahl insbesondere mögliche negative Auswirkungen betreffend Lärmerzeugung, Verkehrsinduktion sowie die soziale Verträglichkeit gegenüber Anrainern zu berücksichtigen. Es wird empfohlen, insbesondere vorliegende Erfahrungen der Stadt Wien mit Erfolgsfaktoren und Hindernissen bei Planung, Anlage und Betrieb von Grill- und Picknickplätzen zu beachten.

C7) Wildlife Viewing-Einrichtungen (Schwarzstorch-Beobachtungswarten): Durch die Einrichtung einzelner, kontrollierter und betreuter Beobachtungseinrichtungen zur Beobachtung des Schwarzstorchs könnten mögliche Nutzungsrestriktionen für Erholungssuchende in Brut- und Nahrungshabitaten des Schwarzstorchs während des Sommers kompensiert werden. Über punktuelle kontrollierte Beobachtungsmöglichkeiten können einerseits Aufklärungs- und Informationsangebote im Sinne des Bildungsauftrags des Biosphärenparks vermittelt und andererseits Nutzungsrestriktionen in der Fläche leichter sowie auf positive Weise kommuniziert werden. In diesem Zusammenhang sollte auch geprüft werden, inwieweit SchwarzstorchHabitate durch Beobachtungseinrichtungen mittels Kameras und Monitoren (gegebenenfalls internetbasiert) zusätzlich geschützt und störungsfrei gehalten werden können.

C8) Verstärkte Instandhaltung und Pflege der bestehenden Wegeinfrastruktur: Untersuchungen haben bestätigt, dass die Bereitschaft zum Verlassen von Wegen im Wienerwald (neben der Wegemarkierung) stark vom Wegezustand und der Attraktivität der Wegeführung abhängt. Je schlechter der Erhaltungszustand von Wegen und je unattraktiver die Wegeführung eingeschätzt werden, desto häufiger geben Erholungssuchende an, die ausgewiesenen Wege zu verlassen (Reimoser et al., 2008). Hieraus folgt, dass Bereiche, in die Erholungsnutzer hin gelenkt werden sollen, durch Investitionen in Wegeerhaltung und ansprechende Wegeführung und -gestaltung gezielt attraktiviert werden können.

\section{Voraussetzungen und Begleitmaßnahmen:}

- Alle vorgeschlagenen kleinräumigen Maßnahmenoptionen zur Besucherlenkung und Funktionstrennung erfordern eine sorgfältige Prüfung der jeweiligen örtlichen Voraussetzungen und Rahmenbedingungen. Dies betrifft insbesondere die vorangehende Bedarfsprüfung sowie die Abschätzung möglicher Risiken und unerwünschter Ausund Folgewirkungen auf Bewohner, Natur, Umwelt und Raum. Wesentlich bei der Planung aller zusätzlichen bzw. alternativen kleinräumigen Freizeitangebote ist ein raumplanerischer Zugang, der auf die Anbindung an vorhandene, nach Möglichkeit öffentliche Verkehrsträger, die Erreichbarkeit von Quellgebieten der Erholungsnutzung (Siedlungsschwerpunkten) aus zu Fuß oder mit dem Fahrrad und die Vermeidung von zusätzlicher Verkehrsinduktion abzielt. Zudem sind bestehende Vorbelastungen (Lärm, Verkehr, etc.) und Belastungsgrenzen für die Anrainer zu berücksichtigen (vgl. auch Maßnahmen K6 und L3).

- Zur Erzielung der erwünschten Lenkungswirkung für Freizeit- und Erholungsnutzungen, d.h. eines Entlastungs- und Beruhigungseffekts in ökologisch sensiblen Gebieten, ist in jedem Fall die Berücksichtigung überörtlicher Zusammenhänge, d. h. des räumlich-funktionalen Zusammenhangs zwischen Attraktivierungsbereichen und DeAttraktivierungsräumen, notwendig. 
- Die selektive Lockerung von zeitlichen Nutzungsrestriktionen für Jogger und Mountainbiker sollte ohne zusätzliche Wegeinfrastruktur, d.h. innerhalb des bestehenden Wegenetzes stattfinden.

- Insbesondere bei neuen Freizeitangeboten, die intensivere Eingriffe in den Naturraum und bauliche Maßnahmen erfordern, und die daher in einem Spannungsverhältnis zu Zielsetzungen einer nachhaltigen Entwicklung des Biosphärenparks stehen, sollten geeignete Standorte außerhalb des Biosphärenparks in die Standortsuche mit einbezogen werden.

- Bei einem Teil der neuen kleinräumigen Freizeitangebote sowie der Liberalisierung von generell im Biosphärenpark bestehenden zeitlichen Nutzungsrestriktionen ist vor der Umsetzung die Klärung haftungsrechtlicher Fragen erforderlich.

- Die Standortwahl sollte bei allen vorgeschlagenen kleinräumigen Maßnahmenoptionen unter besonderer Berücksichtigung der Leitfunktion Erholung gemäß Waldentwicklungsplan erfolgen.

\section{Risiken und Hindernisse:}

- Grundsätzlich ist bei allen Kompensations- bzw. Alternativangeboten, welche die kleinräumig begrenzte Ausübung bestimmter Freizeitaktivitäten auch außerhalb der ansonsten im Biosphärenpark geltenden räumlichen und zeitlichen Nutzungsregeln ermöglichen würden, das Risiko einer weiteren Bedarfsweckung nach Ausübung nicht regelkonformer Aktivitäten (Nachtaktivitäten, Mountainbiking im Winter und abseits von ausgewiesenen Wegen, etc.) bzw. der Verstärkung bestehender diesbezüglicher Nachfragesituationen in der Fläche nicht auszuschließen. Diesem Risiko kann durch differenzierte Kommunikationsstrategien entgegen gewirkt werden. Aus demselben Grund wird von den am Projekt teilnehmenden Stakeholdern der Verzicht auf eine breite Bewerbung zusätzlicher Freizeitangebote befürwortet.

- Ein Hindernis für die Anlage von eigenen Mountainbike-Downhill-Strecken und Technik Trails könnte die hierfür unter Umständen notwendige forstrechtliche Rodungsbewilligung sein. Grundsätzlich sollten derartige Angebote jedoch ohne intensivere Eingriffe in den Waldbestand umgesetzt werden.

\section{D) Verstärkte Information, Kontrolle und Vollzug in Schwerpunktbereichen}

Ziele:

- Förderung von regelkonformem Nutzerverhalten und Verhinderung von Verstößen gegen Verhaltensregeln und Nutzungsrestriktionen in Bereichen mit erhöhtem Management- und Kontrollbedarf

- Verbesserung der Wirksamkeit von Managementmaßnahmen und von Vereinbarungen zu Nutzungsregeln

Handlungsstrategie:

- Effizienter Einsatz von Aufsichts- und Kontrollpersonal durch räumliche Schwerpunktsetzungen 
Maßnahmenoptionen:

D1) Konsequente Durchsetzung des Wegegebotes in Kernzonen: Das derzeit nach dem niederösterreichischen Naturschutzgesetz geltende Wegegebot in den Kernzonen sollte insbesondere bei erhöhter ökologischer Sensibilität sowie bei erhöhtem Konfliktpotenzial durch verstärkte Kontrolltätigkeit von Aufsichts- und Wachorganen konsequenter durchgesetzt werden. Dies gilt in besonderem Maße für Kernzonenbereiche, die von der Indikatorart Schwarzstorch (bzw. anderen naturschutzrechtlich geschützten Tierarten) als Brut- oder Nahrungshabitat genutzt werden, aber auch für diejenigen Kernzonen, die in Jagdkonzepten als Wildruhezonen z. B. für Rotwild vorgesehen sind. Darüber hinaus ist zu empfehlen, die Nutzungsregeln im Wiener Teil des Biosphärenparks an die im niederösterreichischen Teil geltenden Bestimmungen anzugleichen (vgl. Empfehlung A1).

D2) Verstärkte Information, Kontrolle und Sanktionierung in sensiblen Gebieten: Die aktive Informationstätigkeit über bestehende generelle Verhaltensregeln und allfällige weitere kleinräumige Nutzungsrestriktionen (A1 - A5) sowie die Kontrolle von deren Einhaltung sollte insbesondere in den ökologisch hoch sensiblen Gebieten sowie in Räumen mit hohem Konfliktpotenzial schwerpunktmäßig forciert werden. Im Sinne der Empfehlung D1) gilt dies generell auch für ausgewiesene Kernzonen des Biosphärenparks. Die in diesem Bericht enthaltenen GIS-analytischen Kartendarstellungen geben Hinweise auf solche Schwerpunktbereiche. Dies erfordert vor allem eine verstärkte Präsenz von Grundeigentümern und befugten Kontrollorganen vor Ort. Information und Aufklärung sollten dabei Priorität vor Sanktionierungen haben. Bei eklatanten oder hartnäckigen Regelverstößen darf allerdings auch die exemplarische Durchsetzung von Strafen im Rahmen geeigneter Sanktionsmechanismen kein Tabu sein. Dosiert eingesetzte Sanktionen können zur Bewusstseinsbildung betreffend die Existenz von Regeln gegenüber Freizeitnutzern sowie zur Herausbildung sozialer Kontrollmechanismen innerhalb von Nutzergruppen beitragen.

D3) Verstärkte Information, Kontrolle und Sanktionierung im Bereich von Kompensations- bzw. Alternativangeboten: Ein weiterer Schwerpunktbereich von Information und Kontrollen sollte das Umfeld von kleinräumigen Kompensations- und Alternativangeboten für bestimmte Freizeitnutzergruppen bilden (vgl. Empfehlungen C1 - C6). Das Ziel der Informations- und Aufklärungsarbeit in diesen Bereichen besteht vor allem darin, auf den Ausnahmecharakter von punktuell erlaubten, ansonsten jedoch nicht zulässigen Aktivitäten aufmerksam zu machen. Zusatzangebote sind besonders geeignet, um generelle Ver- und Gebote auf positive Weise zu transportieren. Durch eine Konzentration von Maßnahmen der Öffentlichkeitsarbeit in den genannten Bereichen kann insbesondere dem Risiko, dass hierdurch allgemeine Bedürfnisse nach „illegalen“ Aktivitäten in der Fläche stimuliert werden, entgegen gewirkt werden.

\section{E) Teilraumbezogene Beurteilung des Managementbedarfs:}

Ziele:

- Klärung der Zielpriorität und des Managementbedarfs bei unklarer bzw. „intermediärer" Konfliktpotenzialsituation 
- Ermittlung der Maßnahmenausrichtung und -kombination mit der günstigsten Aufwand-Nutzen-Relation

Handlungsstrategie:

- Einzelfallprüfung

Maßnahmenoptionen:

E1) Prüfung des spezifischen Managementbedarfs und situationsabhängige Priorisierung von Handlungsstrategien und Maßnahmen: Insbesondere in Teilräumen mit unklarer Konfliktpotenzialsituation, wo sich weder aus der Sensibilität von Indikatorarten (Vorkommenswahrscheinlichkeit) noch aus der Erholungsnachfrage (Nutzungswahrscheinlichkeit) klare Managementziele und Handlungsorientierungen ableiten lassen, sollte eine spezifische Prüfung der Situation und des Managementbedarfs vorgenommen und allfällige Managementmaßnahmen einer Kosten-Nutzen-Bewertung unterzogen werden. Eine spezifische Prüfung von Konfliktsituation und Managementbedarf ist in Bezug auf jagdbare (indikator)Wildtierarten auch dort angezeigt, wo eine kleinräumige, flexible Maßnahmenabstimmung zwischen Besuchermanagement und Bejagungsmanagement besonders zur Konfliktminimierung beizutragen vermag. Je nach Ergebnis dieser Einzelfallprüfung können unterschiedliche Ziele und Maßnahmen priorisiert oder auch Managementeingriffe unterlassen werden. Hiervon unbenommen bleibt, dass grundsätzlich alle kleinräumigen Maßnahmenoptionen, die auf eine Schaffung zusätzlicher bzw. alternativer Freizeitangebote abzielen (vgl. Empfehlungen C1 C7), einer sorgfältigen Überprüfung der örtlichen Gegebenheiten und Rahmenbedingungen bedürfen. 


\subsubsection{Querschnittsorientierte Handlungsoptionen und Begleitmaßnahmen}

\section{F) Information, Kommunikation, Öffentlichkeitsarbeit}

Ziele:

- Erhöhung der Wirksamkeit von naturraumbezogenen Informationen für Freizeitnutzer

- Besucherlenkung durch zielgruppenspezifische Informationsangebote

Handlungsstrategie:

- Zielgruppenspezifische Informationsangebote und Kommunikationsarbeit

Maßnahmenoptionen:

F1) Zielgruppenspezifische Kommunikation und Öffentlichkeitsarbeit: Wirksame und erfolgreiche Kommunikation von naturraumbezogenen Inhalten und Verhaltensregeln sollte sich an zielgruppenspezifischen Kriterien orientieren. Ein erhöhter Bedarf nach differenzierter Zielgruppenorientierung von Kommunikationsprozessen ergibt sich nicht zuletzt aus der Vielfalt und Heterogenität der unterschiedlichen Freizeitnutzergruppen, wie sie durch die im Projekt verwendeten IndikatorFreizeitaktivitäten repräsentiert werden. Jede dieser Nutzergruppen ist durch spezifische und unterschiedliche demographische und soziale Milieumerkmale, Vorwissen, Interessen und Werte, kommunikative Präferenzen und Gewohnheiten, sprachliche Codes etc. charakterisiert. Diese Unterschiede haben Auswirkungen auf die Auswahl geeigneter und Erfolg versprechender Kommunikationspfade, Vermittlungsorte und Darstellungsformate von Inhalten. Die zu transportierenden Inhalte sind nicht unabhängig von der Wahl der Kommunikationspfade, und beide stehen in enger Wechselwirkung mit den Adressaten und zielgruppenspezifischen Bedürfnissen. In ähnlicher Weise gilt auch für die Personen, die als Vermittler (Sender) von Botschaften agieren, dass sie bestimmte Qualitäten, wie z.B. Glaubwürdigkeit, für die Zielgruppe erfüllen sollten.

Kommunikationsprozesse lassen sich nach Lasswell (in: Schuster et al., 2005) durch folgende Grundfrage beschreiben: Wer sagt was zu wem über welche Pfade und mit welcher angestrebten Wirkung? Bei der Beantwortung jeder einzelnen Frage dieses Schemas sollte die jeweilige Zielgruppe gesondert berücksichtigt werden. Weitere Hilfestellungen bietet die nachstehende Tabelle 39. 
Tabelle 39: Leitfragen zur Planung von zielgruppenspezifischen Kommunikationsprozessen (Quelle: BFN, 2011).

\begin{tabular}{|l|ll|}
\hline Wer ? & - & Wer ist der Sender der Botschaft? \\
& - & Ist der Sender für die Zielgruppe glaubwürdig? \\
& - & Hat der Sender die notwendigen Senderqualitäten für die Zielgruppe? \\
& - & Gibt es geeignete Kooperationspartner, z.B. Vorbilder aus der „Szene \\
& welche die Glaubwürdigkeit der Information erhöhen?
\end{tabular}

Für die zielgruppenspezifische Kommunikation von naturraumbezogenen Inhalten bieten sich insbesondere folgende Kommunikationspfade an (BFN, 2011). Eine Übersicht über die Kommunikationspfade findet sich in Abbildung 115.

F1.1) Interne Kommunikation: Um die Glaubwürdigkeit der transportierten Information zu unterstützen und die jeweilige Zielgruppe effizient zu erreichen, sollte die Kommunikation über die internen Kommunikationskanäle (Homepages, Internetforen, Verbands- oder Vereinsorgane, Mitgliederzeitschriften, Mitgliederveranstaltungen, Sitzungen und Versammlungen, etc.) von Nutzergruppen und deren Vertretungsorganisationen (Verbände, Vereine, etc.) forciert werden. Die Einschleusung der Informationen erfordert die vorangehende enge Kooperation mit legitimierten Vertretern der jeweiligen Nutzergruppen.

F1.2) Aus- und Weiterbildung: Informationen über die Auswirkungen von Freizeitaktivitäten auf Naturraum und Tierarten sowie über diesbezügliche Verhaltensregeln können über bestehende Aus- und Weiterbildungsangebote sowie über Zulassungsbedingungen (Prüfungen) bzw. Kurse für bestimmte Nutzungsformen vermittelt werden. Ein Vorteil hierbei ist, dass die didaktische Qualifikation der vermittelnden Personen ausreichend gewährleistet sein sollte. Wesentliche Voraussetzungen für die Integration der zu vermittelnden Inhalte in bestehende Aus- und Weiterbildungsprogramme (von Schulen, Verbänden, Vereinen, Kursanbietern, etc.) sind dabei die erfolgreiche Überzeugungsarbeit gegenüber den Ausbildungsverantwortlichen sowie die entsprechende inhaltliche Qualifizierung der Vermittlungspersonen. 
F1.3) Kommerzielle Angebote: Hierunter ist die Aufnahme von entsprechenden Inhalten in Angebote, Informationsmaterialien, Events etc. von OutdoorReiseveranstaltern, Sportkursanbietern, Verbänden und Vereinen zu verstehen.

F1.4) Kommunikationsschleusen: Informationen zum richtigen Verhalten im Naturraum können grundsätzlich auf allen Stationen angeboten bzw. mitgegeben werden, durch die alle Naturnutzer „durchgeschleust“ werden, die eine Sport- oder Freizeitaktivität im Naturraum ausüben wollen. Solche Kommunikationsschleusen können die folgenden Stationen umfassen: Vorbereitung (alle digitalen und analogen Infomedien, einschließlich Website, Internetforen, Karten, Routenführer, Fachzeitschriften, Massenmedien, etc.); Ausrüstung (Kauf, Miete, Werbung, Firmen); Anreise und Ankunft (öffentliche Verkehrsmittel, ÖV-Haltestellen, Parkplätze, Gaststätten, etc.); Aufenthalt im Biosphärenpark (Hütten, Besucherzentrum, Info-Tafeln, Karten, GPS, Handy-Apps; Aufsichtspersonen und Kontrollorgane; etc.).

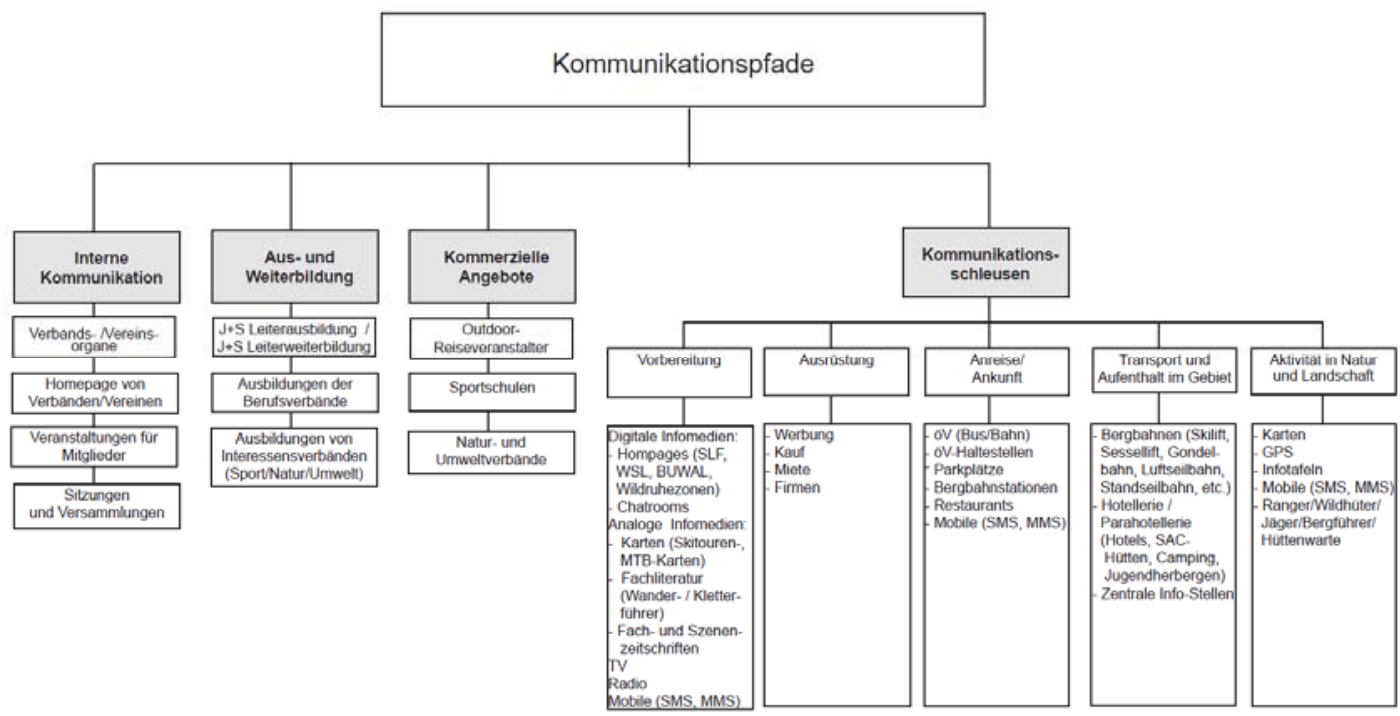

Abbildung 115: Übersicht über wesentliche Kommunikationspfade (Quelle: BFN, 2011)

F2) Erstellung von biosphärenparkweiten und zielgruppenspezifischen Konzepten für Kommunikation und Öffentlichkeitsarbeit: Eine wirkungsvolle Kommunikation und Öffentlichkeitsarbeit sollte in eine biosphärenparkweit einheitliche, langfristige und systematische Kommunikationsstrategie eingebettet sein, die sich gegenüber unterschiedlichen Zielgruppen allerdings spezifischer Kommunikationspfade und -mittel bedienen sollte. Ein diesbezügliches Kommunikations- und Öffentlichkeitsarbeitskonzept sollte von allen relevanten Akteuren im Biosphärenpark gemeinsam erarbeitet und mitgetragen werden.

F3) Schaffung von Kommunikationsstrukturen mit institutionalisierten Vertretern: Von zentraler Bedeutung sowohl für die partnerschaftliche Erarbeitung von Verhaltensregeln und Maßnahmen für das Konfliktmanagement als auch für deren Kommunikation an alle Nutzergruppen ist die Organisation von effizienten, institutionalisierten und verbindlichen Kommunikationsstrukturen innerhalb des Biosphärenparks. Wesentlich hierbei ist, dass alle relevanten Nutzer- und Interessengrup- 
pen im Naturraum (alle Freizeitnutzergruppen, Grundeigentümer und Bewirtschafter, Jägerschaft, Behördenvertreter, Biosphärenparkmanagement, Kommunalpolitik, etc.) einbezogen und vertreten sind. Eine entscheidende Voraussetzung ist neben dem Vorhandensein funktionierender Kommunikationswege innerhalb der einzelnen Nutzergruppen insbesondere die Existenz legitimierter und anerkannter Vertreter aller Gruppen. Als Barriere ist im Biosphärenpark Wienerwald der stark unterschiedliche Organisationsgrad der einzelnen Freizeitnutzergruppen zu werten. Hieraus resultiert, dass in einzelnen, gegenwärtig nicht oder gering organisierten Gruppen legitimierte Vertreter und Ansprechpersonen fehlen. Dies betrifft im Biosphärenpark insbesondere die Gruppen der Mountainbiker, Jogger und Hundehalter. Für diese Gruppen von Freizeitnutzern erscheint die Schaffung von institutionalisierten Vertretern auf Biosphärenparkebene, einschließlich deren Vernetzung mit den zuständigen politischen und verwaltungstechnischen Entscheidungsträgern, vordringlich. Eine Handlungsoption könnte es sein, auf kommunaler Ebene Referenten zu bestimmen, welche die Anliegen und Interessen von Freizeitnutzern und Sportausübenden vertreten.

F4) Zentrale Internet-Marketingplattform für naturbasierte Freizeitaktivitäten: Um das Besucherlenkungspotenzial von digitalen Informationsangeboten für Erholungssuchende besser ausschöpfen zu können, bedarf es einer zentral koordinierten Informationsplattform für den Biosphärenpark im Internet. Aus Gründen der Praktikabilität wird empfohlen, hierzu das bestehende Freizeit- und Sportportal auf der Website des Landes Niederösterreich (http://www.niederoesterreich.at/portal/default.asp?tt=NOE09 R8) zur zentralen Marketingplattform für naturraumbasierte Erholungstätigkeiten im Biosphärenpark auszubauen. Die zentrale Koordination sollte dementsprechend über das Amt der Niederösterreichischen Landesregierung erfolgen. Dies würde auch dem individuellen Freizeitplanungsverhalten besser entgegen kommen als ein auf den Biosphärenpark begrenztes Informationsangebot, weil die Freizeitnutzer ihre Aktivitäten überwiegend nach anderen Kriterien als der Lage innerhalb oder außerhalb der Biosphärenparkgrenzen planen. Wesentlich sind hierbei die zentrale Abstimmung aller ausgewiesenen Freizeitangebote (Mountainbike-Strecken, Geocaching, Laufstrecken, etc.) und die Einbettung in ein stimmiges Marketingkonzept für den gesamten Biosphärenpark. Es sollte angestrebt werden, Sport- und Freizeitinformationen auf der Website des Biosphärenparks, der Stadt Wien sowie auf vorhandenen zivilgesellschaftlichen Internetangeboten einzelner Nutzergruppen so weit wie möglich mit der zentralen Plattform zu verlinken. Bestehendes Optimierungspotenzial in der Darstellung, Vermarktung und Benutzerfreundlichkeit sollte genutzt werden. Die Möglichkeit zur Vermarktung des eigenen Angebotes von Grundeigentümern soll in Abstimmung mit der oben genannten Koordinierungsstelle erhalten bleiben.

F5) Naturraumverträgliches Geocaching-Konzept: Entwicklung eines biosphärenparkweiten Geocaching-Konzepts, das in das bestehende interaktive Web-MapService im Rahmen des offiziellen Sport- und Freizeitportals des Landes Niederösterreich eingebettet werden sollte (siehe oben). Dieses sollte zur zentralen Geocaching-Plattform ausgebaut werden. Eine entscheidende Voraussetzung für die Akzeptanz dieses Informationsangebotes durch die Geocaching-Community ist die Kooperation mit bestehenden zivilgesellschaftlichen Geocaching-Plattformen im Internet. Um Geocaching-Aktivitäten in ökologisch sensiblen, von Freizeitnutzungen möglichst freizuhaltenden Naturräumen im Biosphärenpark hintanzuhalten, sollten diese Gebiete in geeigneter Weise im Geocaching-Konzept ausgeschieden und berücksichtigt werden (vgl. auch Reimoser et al., 2008). 
F6) Verbesserung der Beschilderung: Mangelhafte und nicht eindeutige Beschilderung wird von Freizeitnutzern häufig als einer der Gründe für die Nichteinhaltung von Wegegeboten und Nutzungszeiten angegeben (Reimoser et al., 2008). Die Beschilderungen von räumlichen und zeitlichen Nutzungsge- und verboten (Mountainbike-Strecken, Fairplay-Regeln, etc.) sollten daher verbessert werden, einschließlich der didaktisch zweckmäßigen Gestaltung, Kontrolle und Instandhaltung der Beschilderung. Ausreichende und transparente Beschilderung wird auch eine Voraussetzung für die Durchsetzung des Wegegebots in den (niederösterreichischen) Kernzonen sein.

F7) Digitale Geoinformationen für Wegeverwaltung und Routenplanung: Um sensible Naturräume und damit zusammenhängende Konfliktpotenziale bereits frühzeitig in der Wegeplanung und -erhaltung berücksichtigen zu können, können diesbezügliche Geo-Informationen den Alpin-, Wander- und Sportvereinen zur Verfügung gestellt werden. Durch GIS-basierte Visualisierung von ökologisch sensiblen Gebieten (Kernhabitate, Ruhe- und Rückzugsgebiete, Wintereinstände, Migrationsrouten etc.) bzw. von Teilräumen mit hohem Konfliktpotenzial können die wegeverantwortlichen Akteure für diese Belange sensibilisiert und in die Lage versetzt werden, die besucherlenkende Wirkung von Wegeinfrastruktur zu erhöhen. Ein Beispiel für eine erfolgreiche Umsetzung bietet das Projekt „Digitale Wanderwegeverwaltung" des Deutschen Wanderverbandes (DWV, 2011). Unter besonderer Berücksichtigung des sich ändernden Verhaltens von Freizeitnutzern im Zuge technischer Neuerungen im Bereich von Kartenvisualisierungen und von Routenplanung mit Hilfe von GPS könnten darüber hinaus Kooperationen mit Kartenerstellern von GPS-Geräten eingegangen sowie Handy-Apps zur Routenplanung bereitgestellt werden, in denen primär naturverträgliche Routen empfohlen bzw. naturräumlich sensible Bereiche visuell eingepflegt und als "no go"-Gebiete dargestellt werden. Ein nachahmenswertes Modell ist das Kooperationsprojekt „Naturverträgliches Geocaching“ des DWV mit Herstellern von GPS-Outdoorgeräten (DWV, 2011).

F8) Präsenz der Grundeigentümer: Um zur Sensibilisierung und Bewusstseinsbildung von Freizeitnutzern beizutragen, sollten Grundeigentümer ihre aktive Präsenz gegenüber Freizeitakteuren verstärken und auf diese bewusst mit aufklärenden Informationen (über erlaubtes und unerlaubtes Verhalten, über die sachlichen Gründe für Verhaltensregeln, etc.) zugehen, z. B. in Internetforen und „social media“, auf Sportveranstaltungen, auf Messen von Tourismus- und Freizeitwirtschaft, etc.).

F9) Schulung von Aufsichts- und Kontrollorganen: Kommunikations- und Konfliktmanagementfähigkeiten sollten verpflichtend in Ausbildungs-, Prüf- und Weiterbildungsprogramme aller behördlichen und betrieblichen Aufsichts- und Kontrollorgane aufgenommen werden. Dies gilt insbesondere für alle Wacheorgane und Forstpersonal, z. B. Jagdaufsichtsorgane, Forstschutzorgane, Feldschutzorgane, Bergwacht, Revierförster, Bezirksförster etc.

F10) Fortbildungsangebote: Inhalte zu naturverträglichem Verhalten sollten in Ausund Weiterbildungsprogramme von Schulen, Verbänden, Vereinen, kommerziellen Kursanbietern, etc. für einzelne Sportarten und Freizeitnutzergruppen integriert werden. Von zentraler Bedeutung hierbei ist insbesondere die naturschutzfachliche und ökologische Qualifizierung der jeweiligen Vermittler (Kursleiter) (vgl. auch F1). 
F11) Positive Kommunikation von Restriktionen über (Kompensations-)Angebote: Nutzungseinschränkungen sollten möglichst auf positive Weise, insbesondere über den gleichzeitigen Verweis auf attraktive, alternative (Kompensations-) Angebote, kommuniziert werden.

F12) Verzicht auf Maßnahmen zur Bewerbung und zum Freizeitmarketing: In Gebieten mit hoher Sensibilität (Vorkommenswahrscheinlichkeit von Indikatorarten) und hohem diesbezüglichem Konfliktpotenzial sollte kein gezieltes Marketing von naturraumbasierten Freizeitangeboten stattfinden. Um zu einer nachhaltigen und naturraumverträglichen Ausübung von Freizeitaktivitäten im Biosphärenpark insgesamt beizutragen, gilt dies unabhängig vom Grad der Indikatorarten-abhängigen Sensibilität insbesondere auch für Teilräume mit derzeit noch geringer Erholungsnachfrage. In diesem Zusammenhang wird darauf hingewiesen, dass diese Empfehlung in einem potenziellen Spannungsverhältnis zur Ausweisung ökologisch sensibler Bereiche in zielgruppenspezifischen Besucherlenkungsinstrumenten steht (Risiko kontraproduktiver Wirkungen im Zusammenhang mit Empfehlungen F4 - F7).

\section{G) Kooperative Konfliktlösungsstrategien}

Ziele:

- Lösung von Konflikten infolge von intensiven oder nicht regelkonformen Freizeitaktivitäten in ökologisch sensiblen Räumen

- Vorausschauende Vermeidung der Entstehung von manifesten Konflikten aus latenten Konfliktpotenzialen

- Gewährleistung der Akzeptanz und Einhaltung von gemeinsam erarbeiteten Maßnahmenkonzepten (Wegekonzepte, Nutzungsvereinbarungen, Alternativangebote etc.) durch Beteiligung und Interessenausgleich

Handlungsstrategie:

- Kooperative Aushandlung und Umsetzung von Maßnahmen zur Konfliktlösung und vermeidung (insbesondere betreffend Maßnahmenoptionen A - C, F) unter Einbeziehung aller Akteure

- Gemeinsame, Nutzergruppen übergreifende Erarbeitung von Wegekonzepten, Nutzungsregeln, Alternativangeboten etc.

\section{Erfolgsfaktoren:}

G1) Schlüsselpersonen in Konfliktlösungsprozessen einsetzen: Unter Schlüsselpersonen sind Personen zu verstehen, die maßgeblich zum Zustandekommen von Konfliktlösungen beitragen und eine Rolle als Prozesseigner einnehmen können. Schlüsselpersonen sollten in der Lage sein, einige zentrale Anforderungen zu erfüllen: Neben fachlichen Qualifikationen, wie z. B. Kenntnissen über verwaltungstechnische Abläufe, rechtliche Grundlagen und Wildtierökologie, sollten sie über hohe soziale Kompetenz verfügen; zu letzterem zählen insbesondere Eigenschaften wie Offenheit, Glaubwürdigkeit, Kooperationsbereitschaft und Lernfähigkeit. Im Idealfall handelt es sich um in ortsansässige, allgemein respektierte und in der Region gut vernetzte Personen, die über Glaubwürdigkeit bei betroffenen Nutzergruppen verfügen und auf die institutionellen Strukturen in der Region zurückgrei- 
fen können. Wesentliche Voraussetzungen sind weiters hohes persönliches Engagement und die Bereitschaft zur Übernahme von Verantwortung.

G2) Beachtung von Qualitätsstandards in Beteiligungsprozessen: Partizipative Konfliktlösungsprozesse sollten sich an anerkannten Qualitätskriterien von Beteiligungsprozessen orientieren und geeignete Beteiligungsformen und -methoden anwenden. Hilfestellungen beim Design und bei der Durchführung von Prozessen bieten z. B. die österreichischen Standards der Öffentlichkeitsbeteiligung (BKA \& BMLFUW, 2008), der diesbezügliche Praxisleitfaden (BKA \& BMLFUW, 2011) sowie weitere praxisorientierte Literatur (z. B. Pfefferkorn et al., 2006). Von grundlegender Bedeutung ist insbesondere die frühzeitige Einbeziehung aller relevanten Akteure, die am Konflikt beteiligt, für die Konfliktlösung erforderlich oder anderweitig von Konfliktlösungsmaßnahmen betroffen sind. Der Einbezug von neutralen externen Moderatoren hat sich insbesondere bei langwierigen und komplexen Konflikten mit verhärteten Fronten bewährt (BFN, 2011).

G3) Konsensfähige, nachvollziehbar begründete, und flexible Maßnahmen: In Konfliktlösungsprozessen getroffene Vereinbarungen und Regelungen sind dann konsensfähig, wenn sie einer Befürwortung durch alle relevanten Akteure förderlich sind und eine hohe Akzeptanz zu erzeugen vermögen. Dies ist wiederum die Voraussetzung für die Erzeugung regelkonformen Verhaltens der betroffenen Nutzergruppen. Von großer Bedeutung für Maßnahmen und deren Kommunikation an die betroffenen Zielgruppen sind eine klare und verständliche Formulierung sowie plausible und nachvollziehbare Begründungen. So genügt es beispielsweise nicht, nur die Inhalte von Nutzungsgeboten und -verboten zu kommunizieren, sondern diese sollten auch ökologisch begründet werden, um das notwendige Verständnis zu schaffen. Flexibel und robust ausgestaltete Maßnahmen bieten den Vorteil, dass sie rasch und ohne großen Aufwand an sich ändernde Rahmenbedingungen angepasst werden können (BFN, 2011). Beispielsweise wären allfällige, räumlich begrenzte Nutzungsrestriktionen zum Schutz des Schwarzstorchs im Wienerwald nur während der Anwesenheitszeit dieser Art im Sommer erforderlich.

G4) Gemeinsame und rasche Umsetzung von Maßnahmen: Die Erfolgschancen für die Umsetzung von vereinbarten Konfliktlösungsmaßnahmen steigen, wenn die am Konflikt beteiligten unterschiedlichen Interessen- und Nutzergruppen gemeinsam die Verantwortung für die Umsetzung übernehmen. Dies setzt natürlich die klare Definition von Rollen und Zuständigkeiten im Konfliktlösungsprozess voraus. Eine rasche Umsetzung von Maßnahmen und Vereinbarungen wirkt motivierend, erhöht das Vertrauen der Beteiligten, und ermöglicht, dass dieselben Personen, die sich an der Konfliktlösung beteiligt haben, auch in die Umsetzung eingebunden werden können (BFN, 2011).

\section{H) Kontrolle und Vollzug von Verhaltensregeln}

Ziele:

- Durchsetzung von Verhaltensregeln, Nutzungsrestriktionen und Vereinbarungen

- Verminderung von Konfliktpotenzialen infolge von Verstößen Einzelner gegen Verhaltensregeln

Handlungsstrategie: 
- Kontrolle und Sanktionen, als komplementäre Strategie zu zielgerichteter Information und Kommunikation

Maßnahmenoptionen:

H1) Schaffung von Kontrollmöglichkeiten: Um eine verbesserte Handhabe zur Kontrolle und gegebenenfalls Sanktionierung von jenen Nutzern zu haben, die gegen Wegegebote und andere verbindliche Regeln verstoßen, sollte die vermehrte Kennzeichnung von Geräten wie Mountainbikes in Erwägung gezogen werden. In Analogie zu den im Wienerwald etablierten Pferdemarken wäre ein mögliches System die Fahrradcodierung mittels auch auf Distanz gerätelesbaren Mikrochips, wie es bei Hunden und zur Identifizierung gestohlener Fahrräder in vergleichbarer Weise bereits angewendet wird. Ein bereits bestehendes System, das jedoch noch nicht für solche Zwecke erprobt ist, ist etwa das System „bikefinder®“. Hierbei wird teilweise schon ab Werk der Mikrochip, der eine fälschungssichere ID gespeichert hat, in den Rahmen eingesetzt. Durch die Registrierung nach dem Kauf kann dieser Chip in der Onlinedatenbank mit der Angabe etwa von Rahmennummer, Hersteller, Farbe des Rades registriert werden, damit nachvollziehbar ist, wem das Rad gehört (http://www.bikefinder.de). Das bestehende System müsste jedoch durch die verpflichtende Angabe der Personalien des Radbesitzers ergänzt werden, um so Nutzer, die gegen Verhaltensregeln verstoßen, identifizieren zu können. Um sowohl die Besitzer von unerlaubt freilaufenden Hunden, als auch Mountainbiker auf illegalen Routen mithilfe der Mikrochips identifizieren zu können, ist die Verbesserung der Lesegeräte hinsichtlich deren Reichweite notwendig, woran aber von den Herstellern dieser Lesegeräte bereits gearbeitet wird. Neben dem direkten Erfassen von Nutzern, die gegen Richtlinien verstoßen, durch Kontrollorgane des Biosphärenparks, wäre in sehr sensiblen Bereichen grundsätzlich auch eine Überwachung durch fix montierte Lesegeräte möglich, da diese eine große Anzahl an eingelesenen Codes speichern können; auf diesbezügliche datenschutzrechtliche Probleme, die vorab sorgfältig zu prüfen wären, wird hier ausdrücklich hingewiesen. Ebenfalls aufgrund datenschutzrechtlicher Gründe dürfte das Auslesen dieser Daten und die Ahndung der Verstöße allerdings wie bei Radarboxen im Straßenverkehr nur von Polizeibediensteten erfolgen.

H2) Ausstattung von Kontrollpersonen mit Kompetenzen: Die Effektivität von Kontrollen erhöht sich, wenn die mit Kontrollen Beauftragten bei der Nichteinhaltung von Vereinbarungen oder Regelungen mit Kompetenzen ausgestattet sind, welche die Durchsetzung von Regelungen ermöglichen können. Es wird hier davon ausgegangen, dass das Vorhandensein der Möglichkeit zur Sanktionierung bereits eine starke verhaltensbeeinflussende Wirkung entfaltet; der Einsatz von Bestrafungsmaßnahmen sollte überlegt, unter Einhaltung der „Eskalationsstufenleiter" und primär im Wiederholungsfall erfolgen. Aufklärung und Information sind grundsätzlich Sanktionen vorzuziehen. Der Rahmen für Sanktionen wird grundsätzlich durch die gesetzlich bzw. behördlich vorgesehenen Befugnisse von befugten Kontrollorganen vorgegeben. Darüber hinaus ist die Festlegung weiterer allfälliger Maßnahmen im Rahmen freiwilliger Vereinbarungen, z. B. zwischen Grundeigentümern und Nutzungsbefugten, möglich.

\section{I) Biosphärenparkweite Verhaltensregeln}

Ziele:

- Wirksamere Kommunikation von Verhaltensregeln 
- Förderung regelkonformen Verhaltens von Freizeitnutzern

Handlungsstrategie:

- Verbesserung von Verständlichkeit, Übersichtlichkeit, Akzeptanz und Einhaltung von Verhaltensregeln durch deren biosphärenparkweite Vereinheitlichung

Maßnahmenoptionen:

I1) Einheitliche Rahmen-Nutzungsregeln im Biosphärenpark: Gegenwärtig bestehen für unterschiedliche Gruppen von Erholungssuchenden unterschiedliche informelle Regelwerke („Fairplay Regeln“) für das Nutzerverhalten, insbesondere hinsichtlich der Nutzungszeiten. Eine weitestmöglich einheitliche Gestaltung der Verhaltensregeln für möglichst alle Nutzergruppen würde deren Kommunikation, die Einhaltung und auch deren Übersichtlichkeit sowohl für die Besucher als auch für die zuständigen Kontrollorgane besser gewährleisten. Die Regeln des Biosphärenparkmanagements für das richtige Verhalten in Kernzonen (http://bpww.at/natur/schutzkategorien-schutzgebiete/die-kernzonen/richtigesverhalten-in-den-kernzonen/) sind ein richtiger Schritt in diese Richtung. Wesentlich hierbei ist, dass die Betroffenen in den Entwicklungs- und Formulierungsprozess von einheitlicheren Verhaltensregeln von Beginn an eingebunden werden, um Misstrauen und Vorurteile von NutzerInnen abzubauen und die Akzeptanz bestmöglich zu gewährleisten (vgl. Maßnahmenoptionen G1 - G4, L1 - L3). Dies würde auch die gemeinsame Identität des Biosphärenparks und dessen Erscheinungsbild nach außen verbessern und stärken.

\section{J) Auswahl und Ausgestaltung von Querschnittsmaßnahmen in Abhängigkeit von Einzelfallprüfungen des spezifischen Managementbedarfs}

Ziele:

- Flexibler und situationsabhängiger Einsatz von Maßnahmen der Information, Kommunikation und Öffentlichkeitsarbeit, um die Zielerreichung bei unklaren bzw. „intermediären" Konfliktpotenzialsituationen, die eine Einzelfallprüfung erfordern, zu unterstützen

Handlungsstrategie:

- Abstimmung von Kommunikationsmaßnahmen auf spezifischen Managementbedarf gemäß Ergebnis der Einzelfallprüfung

Maßnahmenoptionen:

J1) Flexible und angepasste Ausrichtung und Anwendung von querschnittsorientierten Kommunikationsmaßnahmen in Sondersituationen: In Bezug auf Teilräume mit unklarer Konfliktpotenzialsituation, in denen eine Einzelfallprüfung des Managementbedarfs (gemäß Empfehlung E1) durchgeführt wird, sollten Maßnahmen dieses Kapitels zur Information, Kommunikation und Öffentlichkeitsarbeit in geeigneter Weise auf das Prüfergebnis und die anderweitige Auswahl spezifischer Managementoptionen abgestimmt werden. 


\subsubsection{Generelle Handlungsstrategien und Maßnahmenoptionen für andere Landnut- zergruppen}

\section{K) Raumplanung, Infrastrukturplanung:}

Ziele:

- Erhaltung von hochwertigen ökologischen Frei- und Grünraumfunktionen durch raumplanerische Flächenvorsorge

- Sicherung und Erhaltung möglichst störungsarmer und großräumig unzerschnittener Lebens-, Rückzugs- und Ruheräume für Wildtierarten

- Erhaltung und Wiederherstellung der ökologischen Landschaftskonnektivität

Handlungsstrategie:

- Konsequente Funktionstrennung zwischen Naturraum und Siedlungsraum

- Verstärkte Berücksichtigung von ökologischen Funktionen und freizeitbedingten Konfliktpotenzialen in den Instrumenten der Raumplanung

\section{Maßnahmenoptionen:}

K1) Forcierte Umsetzung von Zielen einer nachhaltigen Siedlungsentwicklung: Die zu erwartende weitere Bevölkerungszunahme wird im peri-urbanen Bereich des Großraums Wien zu weiterer Zunahme der Siedlungstätigkeit und fortschreitender Suburbanisierung führen (vgl. Kap. 3.1). Da Siedlungsgebiete als Quellgebiet und wesentlicher push-Faktor für Freizeitnutzungen fungieren, ist daher auch von einer Zunahme des diesbezüglichen Nutzungsdrucks im Biosphärenpark Wienerwald auszugehen. Um eine Verschärfung bestehender Konfliktpotenziale, insbesondere in wildtierökologisch sensiblen Räumen, zu vermeiden, kommt bestehenden Zielen einer nachhaltigen Siedlungsentwicklung gerade im Biosphärenpark eine verstärkte Bedeutung zu. Dies betrifft raumordnungsrechtlich verankerte Zielsetzungen wie die die Schaffung klar abgegrenzter, kompakter Siedlungskörper, das Vermeiden von Zersiedelung und die flächensparende Siedlungsentwicklung mit verdichteten Bauformen. Um eine weitere diffuse Suburbanisierung auch in bisher weniger entwicklungsdynamischen Gemeinden des Wienerwalds zu vermeiden, wird zukünftig gerade in der Biosphärenparkregion eine konsequentere und wirkungsvollere Anwendung der Raumplanungsinstrumente gefordert sein. Ansatzpunkte hierfür bieten $u$. a. folgende Handlungsstrategien:

- Forcierter Einsatz des im Niederösterreichischen Raumordnungsgesetz verankerten Instruments der Siedlungsgrenzen im Regionalen Raumordnungsprogramm.

- Konzentration der weiteren Siedlungsentwicklung auf bestehende, gut erschlossene Standorte mit leistungsfähiger öffentlicher Verkehrsanbindung auf der Ebene der überörtlichen Raumplanung.

- Konsequente Umsetzung überörtlicher Planungsvorgaben auf der örtlichen Planungsebene (Flächenwidmungsplanung).

- Rückführung von überhöhten Baulandreserven in den Wienerwaldgemeinden im Zuge von Überarbeitungen der Flächenwidmungspläne. 
K2) Verstärkte Sicherung von störungsarmen, ökologisch bedeutsamen Freiund Grünräumen sowie von ökologischen Korridoren und Migrationsachsen: Um die weitere Verschärfung von Konfliktpotenzialen und Konflikten zwischen Siedlungsentwicklung und hierdurch induzierten Freizeitaktivitäten einerseits und ökologischen Lebensraumfunktionen andererseits hintanzuhalten, sollten die Lebensraumansprüche von Zielarten (z. B. anhand der Indikatorarten dieses Projekts) sowie bestehende Konfliktpotenziale (z. B. anhand der Konfliktpotenzialkarten dieses Berichts) verstärkt in der Raumplanung berücksichtigt werden. Folgende Handlungsstrategien bieten Ansatzpunkte in diesem Sinne:

- Forcierter Einsatz des im niederösterreichischen Raumordnungsgesetz verankerten Instruments der multifunktionalen Grünzonen, die u.a. auch der Absicherung von ökologischen Vernetzungsfunktionen dienen.

- Prüfung der Möglichkeit, darüber hinausgehende, hochwertige ökologische Freiraumfunktionen (insbesondere die Lebensraumfunktion) als eigenständige Nutzungskategorie in der überörtlichen Raumordnung zu verankern, z. B. in Form der Festlegung von Vorrang- und Vorbehaltsflächen für ökologisch bedeutsame Freiräume.

- Festlegung geeigneter und restriktiver Widmungsverbote und -gebote für die örtliche Planungsebene der Gemeinden und konsequenter Vollzug in der Flächenwidmung.

- Bereitstellung von ökologischen Lebensrauminformationen und von Grundlageninformationen zu bestehenden Konfliktpotenzialen als Planungsgrundlage für die örtliche Raumplanung; verpflichtende Berücksichtigung in den örtlichen Landschafts- und Grünraumkonzepten sowie deren Aufwertung im Rahmen der Örtlichen Raumordnungsprogramme.

- Verstärkte Integration von naturschutzrechtlichen Instrumenten (z. B. Kernzonen des Biosphärenparks, Natura 2000-Gebiete, etc.) auf allen Planungsebenen der Raumplanung.

- Erstellung eines ökologischen Freiraumverbundsystems unter besonderer Berücksichtigung von regionalen und überregionalen ökologischen Korridoren sowie die diesbezügliche Flächensicherung über geeignete Planungskategorien in den Raumplanungsinstrumenten.

K3) Errichtung und raumplanerische Absicherung von ökologischen Querungshilfen für Wildtiere: Um die großräumige Lebensraumvernetzung, u.a. für Rotwild, wiederherzustellen, ist insbesondere entlang der Autobahn A21 und im Abschnitt zwischen Hinterbrühl und Gaaden die Errichtung einer oder mehrerer Querungshilfen für Wildtiere (Wildtierpassage, Wildbrücke, Grünbrücke) erforderlich. Bei der Detailplanung sind hinsichtlich der Dimensionierung und baulichen sowie vegetationstechnischen Ausgestaltung die Standards gemäß der 2007 für verbindlich erklärten RVS 04.03.12 Wildschutz (BMVIT \& ÖFGS, 2007) bzw. diesbezügliche Fachgrundlagen in Völk et al. (2001) anzuwenden. Hinsichtlich der Standortwahl sollten vorliegende Kartierungen zu wildökologischen Korridoren herangezogen bzw. vorab Detailkartierungen erstellt werden. Eine wesentliche raumplanerische Begleitmaßnahme zur Funktionssicherung ist die Absicherung von Wildtierpassagen und Korridoren, d. h. das langfristige Freihalten von Bautätigkeiten und intensiven Nutzungen durch geeignete Widmungsbeschränkungen. 
K4) Verstärkte Prüfung von Entwicklungsvorhaben im Rahmen der Anwendung von Prüfinstrumenten: Um weitere Lebensraumverluste und -beeinträchtigungen zu vermeiden und ökologisch bedeutsame, noch weitgehend unzerschnittene Lebensräume möglichst störungsfrei zu erhalten, sollte gerade im Biosphärenpark eine besonders rigorose Prüfung von Projektvorhaben im Rahmen der UVP und der SUP erfolgen. Unabhängig vom jeweiligen Konfliktpotenzial sollte dies genereell für die Erhaltungszone des Biosphärenparks gelten. In den Kernzonen sollten Vorhaben a priori ausgeschlossen sein. Die Durchführung einer Naturverträglichkeitsprüfung (NVP) nach dem Naturschutzrecht in denjenigen Teilen des Wienerwalds, die Natura 2000-Schutzstatus nach FFH- bzw. Vogelschutz-Richtlinie haben, ist als obligatorisch vorauszusetzen. Bundesländergrenzen überschreitende Auswirkungen von geplanten Vorhaben sind bei deren Prüfung gesondert zu berücksichtigen.

K5) Schaffung eines Flächenpools für Ausgleichsmaßnahmen: Insbesondere in den Entwicklungszonen des Biosphärenparks kann und soll auch wirtschaftliche Entwicklung in nachhaltiger Weise stattfinden. Nicht minderbare negative Auswirkungen diesbezüglicher Vorhaben auf die Lebensraumqualität von Wildtierarten bzw. auf damit zusammenhängende Konfliktpotenziale sollten jedoch durch geeignete Ausgleichsmaßnahmen kompensiert werden. Hierfür wird die Schaffung eines Bundesländergrenzen überschreitenden Ausgleichsflächenpools empfohlen. Für den Fall, dass sich die Einrichtung derartiger Flächenpools für Ausgleichsmaßnahmen innerhalb der Bundesländer bzw. bundesweit weiterhin verzögert, sollte die Schaffung eines Ausgleichsflächenpools auf der Ebene des Biosphärenparks, d.h. in jedem Fall unter Kooperation der beiden Bundesländer Niederösterreich und Wien, angestrebt werden.

K6) Aktive und koordinierende Rolle der Raumordnung bei der Standortplanung und Raumverträglichkeitsprüfung von neuen bzw. alternativen Freizeitangeboten: Entsprechend den bei der Maßnahmengruppe $C$ angeführten Voraussetzungen und Begleitmaßnahmen bei zusätzlichen bzw. alternativen Angebotsschaffungen (siehe Empfehlungen $\mathrm{C} 1-\mathrm{C} 7$ ) sollten die raumstrukturelle Verträglichkeit und mögliche negative Auswirkungen diesbezüglicher Standorte, insbesondere auf Verkehrsaufkommen, Lärmbelastungen für Anrainer etc., sorgfältig geprüft werden. Der Raumordnung kommt bei der Standortwahl und deren Beurteilung eine zentrale Rolle zu.

\section{L) Sektorübergreifende Kooperation aller Landnutzungsbereiche:}

Ziele:

- Intensivierung der Zusammenarbeit aller Akteure und Erarbeitung einer gemeinsamen Strategie bei naturraumbezogenen Belangen, wie etwa bei der Erhaltung und Vernetzung von Wildtierhabitaten oder bei der Sicherung und Erhaltung derzeit störungsarmer, ökologisch sensibler Gebiete (vgl. Empfehlungen der Maßnahmengruppen $F$ und $G$ )

- Verstärkte Berücksichtigung von ökologischen Funktionen und freizeitbedingten Konfliktpotenzialen in den Instrumenten aller relevanten Nutzergruppen

- Interessenausgleich bei der Standortfindung und Angebotsentwicklung von kleinräumigen Alternativ- bzw. Kompensationsangeboten zur Besucherlenkung (vgl. Empfehlungen der Maßnahmengruppe C) 
Handlungsstrategie:

- Kontinuierliche Zusammenarbeit und regelmäßiger Informationsaustausch mit allen beteiligten Akteuren zur Optimierung von Synergien zwischen partnerschaftlichen und hoheitlichen Instrumenten

- Aufbau von strategischen Partnerschaften mit betroffenen Nutzergruppen

- Aktive Rolle des Managements Biosphärenpark Wienerwald unter Einbeziehung aller betroffenen Nutzergruppen bei der Ausarbeitung des Managementplanes für den Biosphärenpark Wienerwald

- Partizipative und kooperative Aushandlungsprozesse bei der Entwicklung von Besucherlenkungsansätzen

Maßnahmenoptionen:

L1) Verbesserte und abgestimmte Öffentlichkeitsarbeit, Bewusstseinsbildung, Kommunikation und Information: Da effektive Öffentlichkeitsarbeit ein essentielles Instrument zur Vermittlung von Inhalt und Image von naturraumrelevanten Belangen darstellt, bedarf es einer Verbesserung der Kommunikation, Information und Bewusstseinsbildung nach „außen“ zur Erreichung naturraumbezogener Ziele. So sollte ein Bestreben darin bestehen, die Zusammenarbeit mit allen Betroffenen zu verbessern. Dies kann durch die Intensivierung persönlicher und institutioneller Kooperationen, wie etwa zwischen dem Lebensministerium, Umweltbundesamt, der Niederösterreichischen Naturschutzabteilung, der Wiener Umweltschutzabteilung MA 22, der örtlichen und überörtlichen Raumplanung, den Universitäten, der Österreichischen Bundesforste AG, den NGOs, Nutzergruppenvertretern und Stakeholdern, erfolgen. Zur Verbesserung dieser Zusammenarbeit, sowie zur Erhöhung der Transparenz der geplanten Aktionen und Maßnahmen für Nutzer bedarf es einer verstärkten Kommunikation über „neue“ Medien (Internet- Plattformen, Diskussionsforen). So könnten Seiten wie „www.naturschutz.at“ oder http://bpww.at/ ausgebaut werden, wobei verstärkt auf die Einrichtung von Diskussionsforen Wert gelegt werden sollte. Über diese Plattformen, aber auch mittels Informationstafeln vor Ort und durch Broschüren könnten weiters nutzergruppenbezogene Informationspakete zum Thema des Störungspotentials hinsichtlich Wildtieren an Nutzer herangetragen werden. Bei der Erarbeitung dieser Informationspakete sollten alle Betroffenen mitwirken, um die Akzeptanz von Nutzungsrestriktionen zu steigern. Eine weitere Maßnahme zur Förderung nachhaltiger Nutzung des Biosphärenparks Wienerwald könnte in der Auszeichnung von best- practiseBeispielen von Landwirtschaft, Forstwirtschaft, Jagd und von Privathaushalten bestehen (vgl. Empfehlungen F1 - F12, G1 - G4, I1).

L2) Verstärkte Mitarbeit aller Nutzergruppen bei der Ausarbeitung des Managementplans für den Biosphärenpark Wienerwald: Um im Zuge eines Managementplans eine nachhaltige Entwicklungsstrategie für den Biosphärenpark Wienerwald zu entwickeln, sollten die Nutzergruppen bzw. deren Vertreter in die Erarbeitung von biosphärenparkweiten Verhaltensregeln, Besucherleitsystemen und lenkungsmaßnahmen, miteinbezogen werden, damit die geplanten Maßnahmen auch auf Akzeptanz bei den Betroffenen stoßen und die Regeln befolgt werden. Des weiteren sollte bei der Erstellung des Managementplans verstärkt mit der örtlichen und überörtlichen Raumplanung kommuniziert werden und die Strategische Umweltprüfung für strategische Naturschutzbelange etabliert und genutzt werden. Im Managementplan sollten neben Verhaltensregeln auch sektorenbezogene Zielkataloge für Jagd, Fischerei, Forstwirtschaft, Landwirtschaft und Raumordnung 
entwickelt werden, sowie Pflege- und Schutzprogramme (Entwicklung von Vertragsnaturschutzmodellen, Naturschutzrahmenplänen). Hierzu wäre es notwendig, Instrumente einer naturschutzfachlichen Vorsorgeplanung auf regionaler und überregionaler Ebene zu erarbeiten.

L3) Aktive Beteiligung und Einbeziehung von Landnutzerinteressen an partizipativen Prozesse zur Entwicklung von kleinräumigen Angebotsschaffungen zur Besucherlenkung: Bei allen Vorhaben zur Schaffung von kleinräumigen Kompensations- bzw. Alternativangeboten für Freizeitnutzergruppen (vgl. Empfehlungen C1 - C6) sollten Vertreter aller Landnutzungsansprüche (Freizeitund Erholungsnutzungen, Forst-, Land und Jagdwirtschaft, Naturschutz) im Rahmen kooperativer Planungsprozesse (vgl. Empfehlungen G1 - G4) beteiligt sein und ihre jeweiligen legitimen Interessen und Standpunkte einbringen. Dies schließt mit ein, dass die Auswirkungen von neuen Angeboten auf Lebensräume und Populationen von Wildtierarten sowie auf die jeweiligen Landnutzungsansprüche umfassend geprüft werden. Land- und forstwirtschaftliche Grundeigentümer können zudem Grundeigentum bzw. diesbezügliche Nutzungsrechte in die Standortentwicklung einbringen. Sollten eingriffsintensivere Vorhaben verwirklicht werden, so kommt u. a. dem Naturschutz bei der Flächenauswahl und Gestaltung von ökologischen Ausgleichsmaßnahmen eine zentrale Rolle zu (vgl. auch Empfehlung K5 zur Raumplanung). 


\subsubsection{Monitoring und adaptives Management}

\section{M) Besuchermonitoring}

\section{Grundsätzliche Relevanz:}

In Gebieten, die vielfältigen Nutzungsansprüchen gerecht werden müssen, ist es wichtig, die einzelnen Nutzergruppen mit ihren jeweiligen Ansprüchen an das Gebiet zu kennen und zu verstehen, um eine nachhaltige Nutzung für alle Nutzergruppen zu gewährleisten.

So müssen sowohl die Besucherströme, die zu dem jeweiligen Gebiet führen, als auch die, die innerhalb des Gebiets stattfinden, bekannt sein. Dies inkludiert die Identifizierung, Erfassung und Evaluierung von Quell- und Zielgebieten der Freizeit- und Erholungssuchenden des Gebiets, sowie natürlich auch eine Identifizierung der Freizeit- und Erholungssuchenden selbst. Vielfältige Informationen werden benötigt; diese reichen von sozio-demographischen Beschreibungen bis hin zur Erfassung von Einstellungen und Verhaltensweisen. Letztendlich ist auch die Anzahl der jeweiligen Personen bzw. Personengruppen von hoher Bedeutung.

Die Wirksamkeit von Besucherlenkungsmaßnahmen im Hinblick auf ein nachhaltiges Wildtiermanagement kann nur dann beurteilt werden, wenn gesicherte Informationen zur Einhaltung und Akzeptanz der Besucherlenkungsmaßnahmen vorliegen. Zur objektiven Kontrolle und Überprüfung der Einhaltung und Akzeptanz eines bestehenden Lenkungssystems ist ein objektives, regelmäßiges und systematisches Besuchermonitoring in Form von Besucherbeobachtungen, -befragungen und -zählungen notwendig, das dem aktuellen Stand der Technik entspricht. Daneben können und sollten auch Beobachtungen und Wahrnehmungen anderer Landnutzer zur Evaluierung miteinbezogen werden, die z. B. mittels Experteninterviews erfasst werden können. Die aus dem Monitoring des Besucherverhaltens gewonnenen Erkenntnisse müssen in Folge in die Adaptierung und Verbesserung von Lenkungsmaßnahmen einfließen (Brandenburg, 2001; Reimoser et al., 2008).

Ziele:

- Gewinnung von Informationen über Nutzungsintensitäten und Nutzerverhalten in räumlich-zeitlichen Dimensionen als Grundlage für die Feststellung des Managementbedarfs und für die Entwicklung von Konfliktmanagementstrategien

- Erfolgskontrolle von Maßnahmen zum Konfliktmanagement als Grundlage für die Nachjustierung von Maßnahmen (adaptives Management)

\section{Handlungsstrategie:}

- Entwicklung neuer, kostengünstiger und durchführbarer Methoden zur Erfassung von quantitativen Besucherströmen und deren räumlich-zeitlicher Verteilung im Biosphärenpark

- Gewinnung empirischer Daten zu Besucherströmen und Nutzerverhalten in Schwerpunktgebieten

- Erfassung von wissens-, bewusstseins- und verhaltensbezogenen Informationen zu spezifischen Nutzergruppen 
Maßnahmenoptionen:

M1) Besuchermonitoring mit Hilfe anonymisierter Bewegungsdaten von HandynutzerInnen: In Österreich sind Versuche bzw. Pilotprojekte des Handynetzbetreibers A1-Telekom bekannt, bei denen anonymisierte Bewegungsdaten von HandynutzerInnen, die über die Sendeanlagen dieses Betreibers Daten senden und empfangen, analysiert werden. Diese Analysen beschränken sich bislang auf Siedlungsbereiche mit einer relativ hohen Dichte an Sendeanlagen. Ziel dieser ersten Analysen waren u.a. zeitliche und räumliche Bewegungsmuster bestimmter Bevölkerungsgruppen zu erfassen, z.B. von Kundlnnen eines Einkaufszentrums oder von PendlerInnen (PendlerInnenzustromanalysen). Diese in Österreich neuartige Form, zeitliche und räumliche Bewegungsmuster bestimmter Bevölkerungsgruppen zu erfassen, erscheint auch für den Biosphärenpark Wienerwald interessant. Die Erfassung des zeitlichen und räumlichen Verhaltens von Besucherlnnen im Wienerwald sowie dessen wechselseitige Beziehung mit den räumlichen und zeitlichen Verhaltensweisen von Wildtieren würde eine neue Datengrundlage bieten, um sowohl das Besucher- als auch das Wildtiermanagement zu optimieren. Die Abklärung der Machbarkeit erfordert jedoch die vorangehende Bearbeitung u. a. folgender Fragen:

- Wie können die Anforderungen an den Datenschutz erfüllt werden?

- Mit welcher räumlichen Genauigkeit (Auflösung) wäre ein Besuchermonitoring im Wienerwald möglich?

Im Biosphärenpark Wienerwald, gibt es im Vergleich zu dichter besiedelten Bereichen, etwa dem Stadtgebiet von Wien, weniger Handysendeanlagen, immerhin aber derzeit insgesamt 251 des Betreibers A1-Telekom, davon 123 GSMStandorte und 129 UMTS-Standorte. Dabei ist zu berücksichtigen, dass wahrscheinlich einige Bereiche im Wienerwald aufgrund von Geländemorphologie oder anderer Abschirmungen, etwa von Bäumen oder Gebäuden, ohne ausreichenden Netzempfang sind.

- Zur Validierung dieser in Österreich neuen Methode zur Erfassung von Bewegungsmuster bestimmter Bevölkerungsgruppen ist eine „Eichung“ mit herkömmlichen Methoden zur Besucherzählung und -befragung unerlässlich. Dazu gehören u. a. auch die Berücksichtigung von Kundenprofilen von Handynetzbetreibern sowie von Verhaltensprofilen der verschiedenen Besuchergruppen im Wienerwald.

Diese und andere Fragen sind jedenfalls vorab zu klären, um künftig ein handydatenbasiertes Besuchermonitoring im Wienerwald etablieren zu können, dass kostengünstig, effizient, regelmäßig und möglicherweise genauer ist (zeitlich, räumlich, quantitativ), als das bislang möglich war. Zur Klärung dieser Fragen wäre ein Forschungsprojekt zum Besuchermonitoring im Biosphärenpark Wienerwald anzustreben.

M2) Beobachtungen und Zählungen von Besucherströmen: Verlässliche empirische Monitoringdaten zu Besucherströmen in feinmaßstäblicher Auflösung werden auch zukünftig in erster Linie über „konventionelle“ Techniken des Besuchermonitorings, die auf Beobachtung und Zählung basieren, zu gewinnen sein. Wegen des damit verbundenen Aufwands müssen derartige Monitoringansätze jedoch auf kleinräumig begrenzte Untersuchungsräume und auf Stichproben beschränkt bleiben; die Aussagekraft so gewonnener Daten für den gesamten Biosphärenpark bleibt begrenzt. Es erscheint naheliegend, die Durch- 
führung von Zählungen auf Schwerpunkträume zu konzentrieren; dies können Bereiche mit besonders hohem Konfliktpotenzial, mit besonders hoher ökologischer Störungssensibilität oder auch solche Gebiete sein, für die spezifische Nutzungsregeln vereinbart wurden.

M3) Befragungen: Befragungen mit einem entsprechenden, wissenschaftlich abgesicherten Befragungsdesign sind grundsätzlich geeignet, repräsentative Daten zu Bewusstseinsgrad, Wissens- und Kenntnisstand, Einstellungen, Verhaltenspräferenzen etc. von Freizeitnutzern zu generieren. Solcherart gewonnene Informationen können eine wertvolle Basis für die Planung und Nachjustierung von Management- und Kommunikationsmaßnahmen darstellen. Insbesondere können es die Befragungsergebnisse ermöglichen, Informationsangebote und Kommunikationsarbeit zielgruppenspezifisch zu gestalten und zu transportieren.

\section{N) Erfolgskontrolle zu vereinbarten Nutzungsregeln}

Ziele:

- Kontrolle und Sicherstellung der Wirksamkeit von kleinräumig begrenzten Maßnahmen, die im Rahmen von Vereinbarungen festgelegt wurden

- Gewinnen einer objektiven Informationsgrundlage zur Anpassung der Maßnahmen

\section{Maßnahmenoptionen:}

N1) Monitoring der Wirksamkeit von getroffenen Nutzungsvereinbarungen: Um die Wirksamkeit von vereinbarten Maßnahmen, einschließlich möglicher Nutzungsrestriktionen (vgl. Empfehlungen A3 - A5), sicherstellen zu können, ist es wichtig, die Einhaltung der Regelungen zu kontrollieren. Die räumliche und zeitliche Maßstabsebene, der zu überprüfende Sachverhalt und die dazu jeweils am besten geeigneten Methoden hängen stark vom jeweiligen Anlassfall ab. Im Regelfall wird sich das Monitoring auf die Einhaltung der Nutzungsregelungen durch die Freizeitnutzer sowie auf die angestrebten Auswirkungen auf Wildtierverhalten und verteilung beziehen. Generelle Erfolgsfaktoren umfassen die Festlegung eindeutiger Zuständigkeiten für die Durchführung der Erfolgskontrolle (im Idealfall die an der Ausarbeitung der Maßnahmen beteiligten Akteure selbst), die Ausstattung von Kontrollpersonen mit Kompetenzen sowie den regelmäßigen Erfahrungsaustausch zwischen allen beteiligten Akteuren nach Abschluss einer Monitoringperiode. Dies inkludiert insbesondere die gemeinsame Diskussion und Bewertung der Monitoringergebnisse und gegebenfalls die Anpassung der betreffenden Nutzungsvereinbarungen.

\section{O) Monitoring von Wildtierpopulationen und -lebensräumen:}

Ziele:

- Gewinnung von zuverlässigen und aktuellen Informationen über Zustand und Entwicklungsdynamik von Wildtierpopulationen, Lebensräumen und über wechselseitige Einflüsse zwischen Wildtieren und Landnutzungsansprüchen

- Schaffung von Daten- und Informationsgrundlagen zur Feststellung des Managementbedarfs, zur Entwicklung von Managementstrategien und -maßnahmen sowie zu deren laufender Überprüfung und Nachjustierung (adaptives Management)

\section{Handlungsstrategie:}


- Einrichtung von Monitoringsystemen zur Beobachtung von Wildtierpopulationen, Lebensräumen und Mensch-Wildtier-Interaktionen

\section{Maßnahmenoptionen:}

01) Einrichtung eines regelmäßigen Schwarzstorch-Monitorings: Basierend auf der 2001 erstmals durchgeführten Bestandserfassung der SchwarzstorchBrutpopulation im Wienerwald (Frank \& Berg, 2001) sollte ein regelmäßiges Monitoring von Habitaten, des Brutbestandes und des Bruterfolges eingerichtet werden. Auf Basis hierdurch gewonnener Daten können indirekt auch die Auswirkungen von Störungen durch Freizeitaktivitäten auf die Bestandsentwicklung abgeschätzt werden. Ein systematisches Monitoring des Schwarzstorchs ist insbesondere erforderlich, um zukünftig die Wirksamkeit eines anzustrebenden Managementplanes und Horstschutzprogrammes für den Schwarzstorch einschätzen zu können.

O2) Fortführung des Ziesel-Monitorings: Die regelmäßige Bestandserfassung der Zieselpopulation auf der Perchtoldsdorfer Heide hat sich als Grundlage zur Vereinbarung von Regelungen zum Konfliktmanagement bewährt und ist unbedingt fortzuführen.

03) Monitoring von Schalenwildeinfluss auf die Waldvegetation: Unter den Indikator-Arten dieses Projekts betrifft diese Empfehlung unmittelbar vor allem das Rotwild. Es gibt bereits Monitoringverfahren, die Jagd-Wald-Wild-Interaktionen dokumentieren. Diese wurden vor allem zur Erkennung und Bewertung von Wildschäden an der Waldvegetation (Verbiss, Schälung, Fegen) und für die Abschussplanung beim Schalenwild verwendet. Der Einsatz und die Weiterentwicklung solcher Verfahren werden im Jagdlichen Leitbild des Biosphärenparks gefordert. Nachdem es zwischen den Nutzergruppen der betroffenen Güter im Schadensfall zu Konflikten kommen kann, wird die Koordination der Verfahren durch den Biosphärenpark empfohlen.

Derzeit nähert man sich dem Wald-Wild-Monitoring auf unterschiedlichen Ebenen. In den Kernzonen wird bereits seit 2007 ein Basis-Monitoringprogramm umgesetzt. Das Konzept für eine Ersterhebung wurde vom Biosphärenpark Wienerwald Management in Zusammenarbeit mit der ÖBf AG erstellt; verschiedene Forschungseinrichtungen wurden beratend beigezogen. Das Basis-Monitoringprogramm erlaubt die unmittelbare Einschätzung des Wildeinflusses derzeit jedoch nur durch die Erhebung des Leittriebverbisses in der Verjüngung. Langfristig kann der Wildeinfluss eventuell auch über Veränderungen in der Baumartenzusammensetzung interpretiert werden. Erhebungen, die darüber hinausgehen sind jedoch derzeit nicht geplant. Wünschenswert wäre ein Erhebungsprogramm, das die umfassende Evaluierung von Abhängigkeiten und Einflussgrößen zwischen Vegetation und Wild bzw. die Quantifizierung von Wildeinflüssen auf die vegetativen Prozesse ermöglicht. Dies kann unter anderem dazu beitragen, zu klären, welche Konsequenzen vegetativer Prozessschutz in den Kernzonen hat, und inwieweit er durch Wildtiere beeinflusst wird.

Zur objektiven Erfassung von Wildeinflüssen auf die Vegetation wären insbesondere ausgewählte Kontrollzaunflächen geeignet, die stichprobenartig auf der ganzen Biosphärenparkfläche verteilt sein sollten. Diesbezügliche Planungen bestehen derzeit seitens des Biosphärenpark Wienerwald Managements nicht. Hingegen besteht in Niederösterreich ein österreichweit abgestimmtes WildeinflussMonitoring-System, das alle drei Jahre Ergebnisse auf Bezirksebene erbringt. 
Schließlich wird der Status quo spezifischer Wildeinflüsse durch ein Jungwuchsund Schälmonitoring bei den ÖBf und in anderen größeren Forstbetrieben mit betriebsspezifisch unterschiedlichen Methoden erhoben. Längerfristige Änderungen der Wildeinflüsse werden alle zehn Jahre bei der Forsteinrichtung größerer Betriebe (z. B. ÖBf 2008/2009) analysiert. In einem sehr weitmaschigen Stichprobennetz wird der Zustand des Waldes (und damit auch die Wildeinflüsse) auch durch die Waldinventur des Bundesforschungs- und Ausbildungszentrums für Wald, Naturgefahren und Landschaft aufgenommen. Die Waldinventur bietet auch überregionale Vergleiche an; lange Zeitserien ermöglichen dazu die Abbildung von Entwicklungstrends.

04) Monitoring von Wildtierbeständen, Wildabschüssen und Fallwild: Eine konsequente Weiterführung der revierbezogenen jagdlichen Abschussstatistik (jährliche Dokumentation anhand standardisierter Abschusslisten) eignet sich, bei Berücksichtigung bekannter Fehlerquellen, gut für die Ableitung langfristiger Bestandsentwicklungstrends bejagter Wildarten. Dies betrifft hinsichtlich der im Projekt IESP ausgewählten Indikator-Arten insbesondere das Rotwild, das Schwarzwild sowie - im Falle einer erfolgreichen Wiederansiedlung - das Auerhuhn. Betriebsspezifische Erfassungs- und Dokumentationssysteme sollten aufeinander abgestimmt werden, um eine gute Vergleichbarkeit zu erreichen.

O5) Zusätzliches Monitoring spezifischer Wildtierindikatoren: Der Biosphärenpark beherbergt eine beachtliche Anzahl unterschiedlicher Wildarten. Das potenzielle Wildarteninventar ist mit Ausnahme weniger Arten (z. B. Fischotter) relativ gut bekannt. Aus Zustands- oder Bestandsänderungen der beobachteten Arten / Artengruppen können Rückschlüsse auf Qualität und Quantität gezielter Veränderungen anthropogenen Ursprungs (z. B. jagdliches Management) gezogen werden. Die Bewertung gemessener Veränderungen gegenüber dem Ausgangszustand erfolgt unter Nutzung indikativer Eigenschaften; die Arten fungieren als Indikatoren. Durch regelmäßige Erfassung spezieller Indikatorarten (Monitoring) kann auf unnatürliche, direkte und indirekte interspezifische Konkurrenzverhältnisse zwischen Wildarten hingewiesen werden. Analog zum Wildschadensmonitoring kann durch gezieltes Management unerwünschten Entwicklungen gegengesteuert werden. Je nach Erfassungsmethode (qualitative, semiquantitative oder quantitative Erhebungen) und Erfassungszeitraum bzw. Erfassungsintervallen werden Aussagen unterschiedlicher Präzision möglich. Beispiel für ein derartiges Monitoring könnte z. B. die Erfassung von Waldschnepfen als Indikator für die Schwarzwilddichte sein.

Das Ziel einer nachhaltigen Jagd sind artenreiche Reviere. In der Realität sind die Abundanzverhältnisse der Wildarten meist zugunsten der ökonomisch interessanten Taxa verschoben. Im Zuge der Entwicklung nachhaltiger Jagd sieht das Jagdliche Leitbild die Unterstützung von Artenschutzprojekten vor. Besondere Erfahrungswerte können Jäger im Speziellen bei Schutzmaßnahmen für im Jagdgesetz geregelte Wildarten einbringen (z. B. Waldschnepfe, Haselhuhn, etc.). Die Förderung sensibler Wildarten ist jedenfalls ein aktiver Beitrag zur Nachhaltigkeit der Jagd.

06) Integration eines Wildtiermonitorings in das allgemeine Biodiversitätsmonitoring: $\mathrm{Zu}$ einem allgemeinen Monitoring der Biodiversität in Österreich gibt es Vorarbeiten und Konzepte (Holzner et al., 2006), die Umsetzung befindet sich in Entwicklung. Ziel dabei ist die Beurteilung der Biodiversität der offenen Kulturlandschaft Österreichs auf allen Skalenebenen, die u. a. für die Überprüfung von 
Schutzzielen zur Biodiversität (2010-Ziel: „Stop of the loss of biodiversity“) dienen soll. Als zentrale Datenbasis ist dabei ein Netz von Stichprobenflächen als repräsentative Dauerbeobachtungsflächen vorgesehen (Österreichische Kulturlandschaftsinventur ÖKI). Dieses Netz und die dort geplanten Erhebungen sind zunächst in einer Dichte vorgesehen, die Aussagen für ganz Österreich erlauben soll. Um regionalspezifische Entwicklungen zu beobachten, wären dann Verdichtungen möglich, die in einem gemeinsamen System mit dem österreichweiten Netz erhoben werden und damit auch vergleichbar wären. Ein zukünftiges wildökologisches Monitoring (siehe oben) sollte deshalb auch koordiniert sowohl mit dem allgemeinen Biodiversitätsmonitoring als auch mit der Waldinventur entworfen werden.

07) Monitoringpflicht in Natura 2000-Gebieten: Die Europäische Kommission schreibt für das Natura 2000-Netzwerk ein kontinuierliches Monitoring für Anhang I-Arten der FFH- und der Vogelschutz-Richtlinie vor. In diesem Kontext sollten wildtierökologische Monitoringprogramme neben der Abstimmung mit dem Datenmanagement des Biosphärenparks unbedingt auf das Natura 2000-Monitoring Rücksicht nehmen.

08) Monitoring der Wiesen: Im Jahr 2007 hat das Biosphärenpark-Management gemeinsam mit den Österreichischen Bundesforsten und dem Naturschutzbund NÖ ein Monitoringprogramm für insgesamt 464 Wienerwald-Wiesen ins Leben gerufen. Ziel ist auf rund 740 ha eine flächenscharfe Kartierung des Pflanzeninventars. Das Monitoring, das auch durch die Länder Wien und Niederösterreich unterstützt wird, umfasst unter anderem das Ausmaß an Flurschäden durch Schwarzwild.

In einem Projekt des Lebensministeriums werden zurzeit die Möglichkeiten untersucht, über Berichte von Landwirten Daten für ein Biodiversitätsmonitoring im Grünland zu sammeln. Artenvorkommen zumindest von charismatischen, auffälligen Arten sollen dabei durch zufällige Beobachtungen, und nicht durch systematische Erhebungen, ermittelt und zentral dokumentiert werden. Dadurch soll eine zwar unsystematische, aber sehr viel breitere Datenbasis erreicht werden, als dies über gezielte Stichprobenerhebungen möglich ist. 


\subsection{Verknüpfung von Maßnahmenoptionen mit Konfliktpotenzialen}

\subsubsection{Erläuterungen zur GIS-Modellierung der Konfliktpotenzialstufen}

In den nachfolgenden Kartendarstellungen der Konfliktpotenziale zwischen jeder der Indikatorarten und der Summe aller Freizeitaktivitäten (siehe Kap. 4.3.2.1.1, Kap. 4.3.2.2.1, Kap. 4.3.2.3.1, Kap. 4.3.2.4.1) wurde die stufenlose Standardisierung der Konfliktpotenziale zwischen 0 und 1, wie sie in den Kartendarstellungen des Kapitels 3.4.3 vorgenommen wurde, auf diskrete Skalen mit - je nach Indikatorart - neun, sechs oder drei Skalenstufen des Konfliktpotenzials umgerechnet.

Die betreffenden Kartendarstellungen des Konfliktpotenzials im vorstehenden Kap. 4.3 (Abbildung 116, Abbildung 118, Abbildung 120, Abbildung 122) kombinieren die Nutzungswahrscheinlichkeit (Summe aller Freizeitaktivitäten) mit der Vorkommenswahrscheinlichkeit (Verteilung und Raumnutzung) der jeweiligen Tierart. Hierfür wurden die Nutzungswahrscheinlichkeiten aller Indikatoraktivitäten für jede Kartendarstellung jeweils linear in drei Werteabschnitte, d.h. in drei gleich große Skalenintervalle (hoch, mittel, gering), klassifiziert. Die Klassifizierung der Vorkommenswahrscheinlichkeiten der Tierarten richtet sich grundsätzlich nach den Abstufungen der Verbreitungskarten (siehe Kap. 3.4.2). Die Vorkommenswahrscheinlichkeiten der Indikatorarten Schwarzstorch und Rothirsch wurden in jeweils drei Stufen (hoch, mittel, gering) klassifiziert; hingegen wird bei der Indikatorart Wildschwein aufgrund der flächendeckend hohen Vorkommensdichte die Vorkommenswahrscheinlichkeit nur in zwei Klassen (Hauptvorkommen - hoch, Nebenvorkommen - mittel) angegeben. Beim Auerhuhn wurden die Kategorien historische Vorkommen, rezente Sichtungen und potenzielle Lebensraumeignung gleichermaßen als Indiz für vorhandenes Wiederansiedlungspotenzial gewertet und keine weitere Klassifikation der Skala vorgenommen, wodurch sich hier nur eine Klasse (Wiederansiedlungspotenzial vorhanden) ergibt.

Zur Ermittlung der Konfliktpotenziale in Bezug auf jede der Indikatorarten wurden die Vorkommenswahrscheinlichkeiten und die Nutzungswahrscheinlichkeiten derart miteinander kombiniert, dass jede Kombinationsmöglichkeit eine eigene Farbgebung erhält. Jede Farbgebung entspricht einer bestimmten Konfliktpotenzialstufe und ergibt sich durch die spezifische Kombination einer bestimmten Nutzungswahrscheinlichkeit mit einer bestimmten Vorkommenswahrscheinlichkeit. Hieraus ergeben sich beim Schwarzstorch (Kap. 4.3.2.1) und beim Rothirsch (Kap. 4.3.2.2) jeweils neun Konfliktpotenzialklassen, beim Wildschwein (Kap. 4.3.2.3) und beim Auerhuhn (Kap. 4.3.2.4) hingegen nur sechs bzw. drei Klassen des Konfliktpotenzials mit jeweils eigener Farbgebung (siehe Verknüpfungsmatritzen in Abbildung 117, Abbildung 119, Abbildung 121 und Abbildung 123).

Die bis zu neun entstehenden Wertekombinationen stellen somit unterschiedliche Konfliktpotenzialsituationen dar, die es ermöglichen, unterschiedliche Typen von Konfliktsituationen räumlich zu differenzieren. Weiters bieten sie die Möglichkeit, das Zustandekommen von Konfliktpotenzialsituationen analytisch näher zu betrachten, vorrangige Ziele für das Konfliktmanagement abzuleiten, sowie aus dem gesamten Maßnahmenportfolio (siehe Kap. 4.2) prioritäre Managementmaßnahmen für unterschiedliche Konfliktkonstellationen auszuwählen und diese über die Karten grob den entsprechenden Konflikträumen zuzuordnen (siehe Kap. 4.3.2). 


\subsubsection{Verknüpfung von Maßnahmenoptionen mit Konfliktpotenzialausprägungen und Konfliktpotenzialräumen in Bezug auf die Indikatorarten}

In diesem Kapitel 4.3.2 wird ein Ansatz vorgestellt und konkret ausgearbeitet, bei dem die im Maßnahmenkatalog des Kapitels 4.2 aufgelisteten und beschriebenen Maßnahmenoptionen unterschiedlichen Abstufungen der Konfliktpotenziale zugeordnet werden. Hierbei wird von den einzelnen Indikator-Tierarten - Schwarzstorch, Rothirsch, Wildschwein und Auerhuhn ausgegangen, weil sowohl die Konfliktpotenziale mit Freizeitaktivitäten als auch die Ziele und Maßnahmen des Konfliktmanagements jeweils stark von Eigenschaften der Indikatorarten abhängen.

Hierbei wird von folgenden Prämissen ausgegangen: Aus tierartenbezogener Sicht repräsentieren die Indikatorarten die Seite der Störungssensibilität, während die IndikatorFreizeitaktivitäten die Seite der Störungsintensität infolge Erholungsnachfrage repräsentieren. Jede Kombination einer bestimmten Störungssensibilität mit einer bestimmten Erholungsnachfrage in einem Teilraum des Biosphärenparks entspricht einer spezifischen Konfliktpotenzialausprägung, die wiederum spezifischen Managementbedarf nach sich zieht. Je nach Indikatorart und spezifischer Konfliktpotenzialsituation können daher unterschiedliche Maßnahmenoptionen aus dem gesamten Maßnahmenkatalog des Kapitels 4.2 besonders geeignet sein, zum Konfliktmanagment beizutragen. Die Zuordnung prioritärer Maßnahmenoptionen zu unterschiedlichen Konfliktpotenzialausprägungen soll in diesem Sinne die Auswahl und Kombination zielführender Managementmaßnahmen erleichtern. Die sorgfältige und detaillierte Prüfung jeder Einzelmaßnahme sowie die kleinräumige Detailplanung in Abhängigkeit von den jeweils besonderen Ausgangs- und Rahmenbedingungen sind in jedem Fall notwendig.

Die Handlungsoptionen für das Konfliktmanagement werden getrennt nach Indikatorarten behandelt. Die Gliederung für jede der Indikatorarten Schwarzstorch, Rothirsch, Wildschwein und Auerhuhn folgt jeweils dem gleichen Muster und besteht aus folgenden Unterkapiteln:

- Modellierung des Konfliktpotenzials im GIS

- Verknüpfungsmatrix (Vorkommenswahrscheinlichkeit mit Nutzungswahrscheinlichkeit)

- Generelle Ziele für das Konfliktmanagement in Bezug auf die Indikatorart

- Zuordnung von Leitfunktionen und Managementzielen zu Konfliktpotenzialstufen

- Übersicht über besonders geeignete Handlungsoptionen (Besuchermanagement, Querschnittsmaßnahmen) je Konfliktpotenzialstufe

- Spezifische Maßnahmenoptionen für weitere Landnutzergruppen in Bezug auf die Indikatorart

- Zuordnung der Maßnahmenoptionen für Landnutzergruppen zu den Konfliktpotenzialstufen. 


\subsubsection{Schwarzstorch}

Die Hinweise und Entscheidungshilfen dieses Kapitels 4.3.2.1 zu den Zielen und zur Maßnahmenauswahl für das Konfliktmanagement beziehen sich ausschließlich auf die Indikatorart Schwarzstorch. Es ist darauf hinzuweisen, dass jedes Konfliktmanagement in konkreten Teilräumen des Biosphärenparks stets auch Konfliktpotenziale und Managementbedarf in Bezug auf die anderen Indikatorarten berücksichtigen und auf deren Zusammenschau basieren sollte.

\subsection{Modellierung des Konfliktpotenzials im GIS}

Die nachfolgende Kartendarstellung (Abbildung 116) zeigt neun unterschiedliche Ausprägungsformen des Konfliktpotenzials zwischen der Indikatorart Schwarzstorch und der Summe aller Indikator-Freizeitaktivitäten im Biosphärenpark Wienerwald. Das Konfliktpotenzial ergibt sich aus der Kombination der summierten Nutzungswahrscheinlichkeit aller Freizeitaktivitäten mit der Vorkommenswahrscheinlichkeit des Schwarzstorchs.

Die Bezeichnung „-wahrscheinlichkeit“ im Zusammenhang mit Indikatorart und Indikatoraktivitäten wird verwendet, um auszudrücken, dass es sich um GIS-Modellierungen handelt, die neben empirischen Daten auch auf theoriegestützten Annahmen beruhen.

Für die Modellierung im GIS wurde die stufenlose Standardisierung der Konfliktpotenziale zwischen 0 und 1, wie sie in den Kartendarstellungen des Kapitels 3.4.3 vorgenommen wurde, auf eine diskrete Skala mit drei Werteklassen des Konfliktpotenzials umgerechnet. Hierfür wurde die Nutzungswahrscheinlichkeit der Indikatoraktivitäten linear in drei Werteabschnitte, d.h. in drei gleich große Skalenintervalle (hoch, mittel, gering), klassifiziert. Die Vorkommenswahrscheinlichkeit des Schwarzstorchs beruht auf der Verteilung und Dichte von Nachweisen (Beobachtungen); die Klassifikation in drei Werteklassen (hoch, mittel, gering) folgt grundsätzlich der Abstufung, wie sie bei der Erstellung der Verbreitungskarte in Kap. 3.4.2.4 (Abbildung 86) verwendet wurde.

Durch die Kombination von drei Klassen der Nutzungswahrscheinlichkeit mit drei Klassen der Vorkommenswahrscheinlichkeit des Schwarzstorchs ergeben sich insgesamt neun Kombinationsmöglichkeiten, die unterschiedlichen Ausprägungsformen des Konfliktpotenzials entsprechen. Jeder der neun Konfliktpotenzialausprägungen wird ein eigener Farbwert zugewiesen. In denjenigen Bereichen des Biosphärenparks, wo keine Sichtungen des Schwarzstorchs vorlagen, besteht hiernach auch kein Konfliktpotenzial mit Freizeitaktivitäten; diese Bereiche sind in der Karte weiß dargestellt. Die Kombination erfolgt über die Verknüpfungsmatrix in Abbildung 117 mittels der darin festgelegten Verknüpfungsregeln.

Die Karte in der nachstehenden Abbildung 116 zeigt die Lage und räumliche Verteilung der neun Konfliktpotenzialstufen im Biosphärenpark. In den nachfolgenden Kapiteln werden den unter-schiedlichen Konfliktpotenzialausprägungen differenzierte Managementziele sowie besonders geeignete Maßnahmenoptionen für das Besuchermanagement und für weitere spezifische Landnutzergruppen zugeordnet. Über die Karte in Abbildung 116 ist näherungsweise die räumliche Zuordnung von vorrangigen Zielen und Maßnahmenoptionen des Konfliktmanagements zu Konflikträumen unterschiedlicher Konfliktpotenzialstufe möglich. 


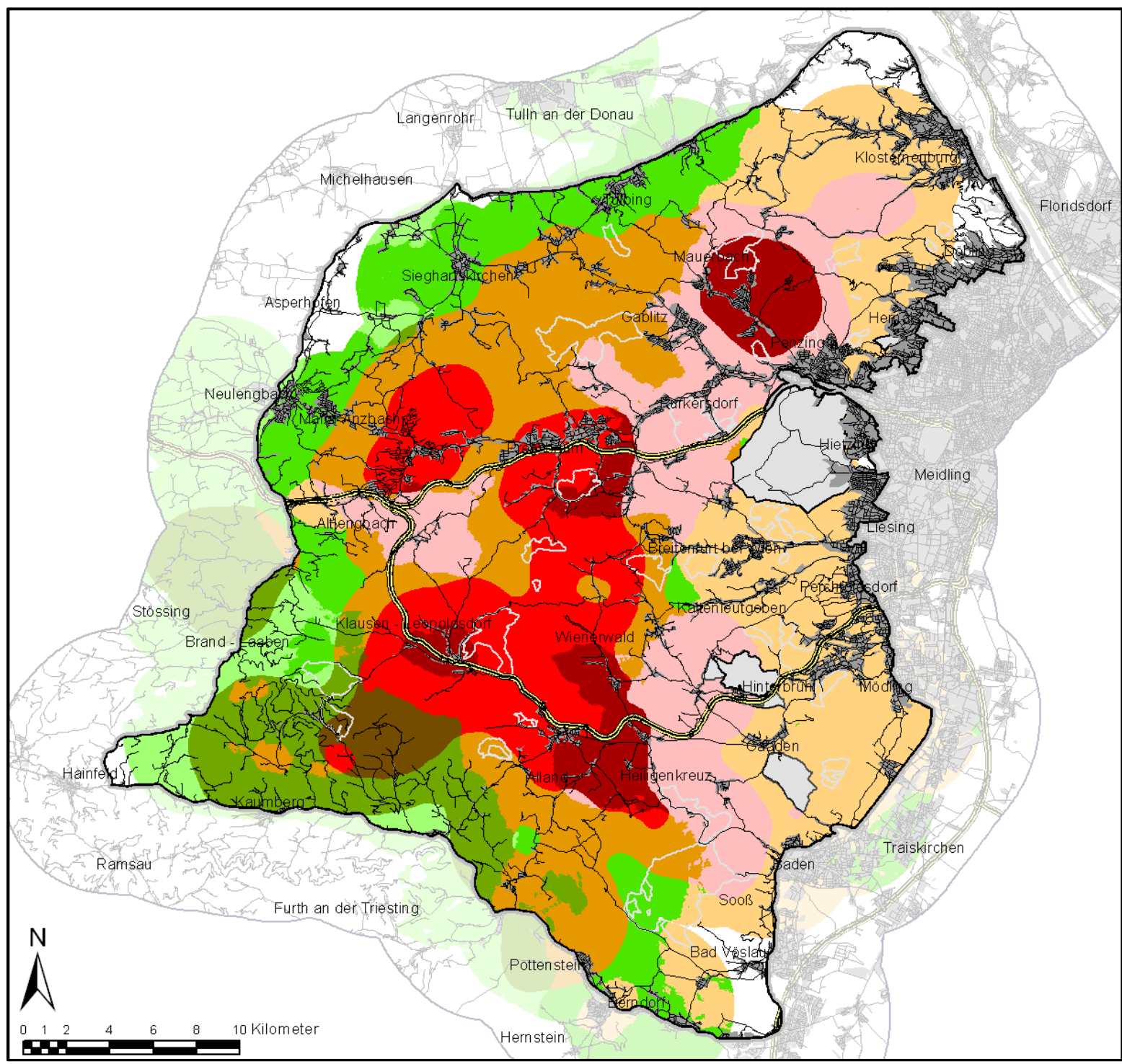

\section{Schwarzstorch:}

Konfliktpotenzial

Vorkommenswahrscheinlichkeit der Tierart mit der

Summe aller Nutzungswahrscheinlichkeiten

Schwarzstorch (SST)

$\square$ SST gering - Aktivitäten gering

SST gering - Aktivitäten mittel

SST mittel - Aktivitäten gering

SST gering - Aktivitäten hoch

$\square$ SST mittel - Aktivitäten mittel

SST hoch - Aktivitäten gering

$\square$ SST mittel - Aktivitäten hoch

SST hoch - Aktivitäten mittel

SST hoch - Aktivitäten hoch

$\square$ keine SST Nachweise

$\square$ Gatter

Kemzonengrenzen
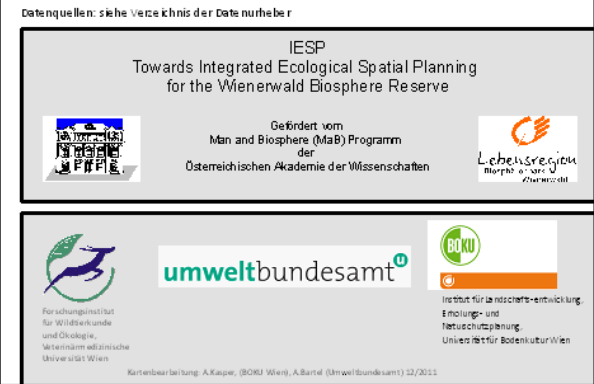

Abbildung 116: Räumliche Verteilung des Konfliktpotenzials zwischen dem Schwarzstorch und der Summe aller Freizeitaktivitäten in neun Werteklassen. 


\subsection{Verknüpfungsmatrix Schwarzstorch}

Mittels nachstehender Abbildung 117 wird die Vorkommenswahrscheinlichkeit des Schwarzstorchs mit der summierten Nutzungswahrscheinlichkeit aller Indikator-Freizeitaktivitäten verknüpft. Über die aus der Abbildung ersichtlichen Vernüpfungsregeln ergeben sich neun Kombinationsmöglichkeiten, die unterschiedlichen Ausprägungsformen bzw. Stufen des Konfliktpotenzials entsprechen. Die Verknüpfungsmatrix erleichtert die Zuordnung von Zielen für das Konfliktmanagement (siehe Kap. 4.3.2.1.4) sowie von besonders geeigneten Maßnahmenoptionen (siehe Kap. 4.3.2.1.5 und Kap. 4.3.2.1.7) zu unterschiedlichen Konflikt(potenzial)situationen. Über die zugewiesenen Farbwerte wird weiters die näherungsweise räumliche Zuordnung von prioritären Managementoptionen in der Karte in Abbildung 116 (Kap. 4.3.2.1.1) möglich.

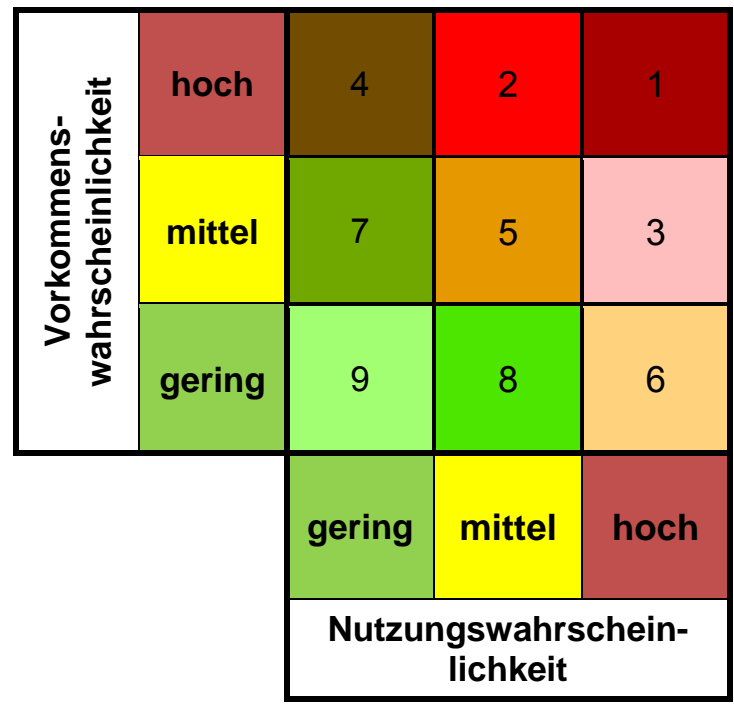

Ausprägung Konfliktpotenzial

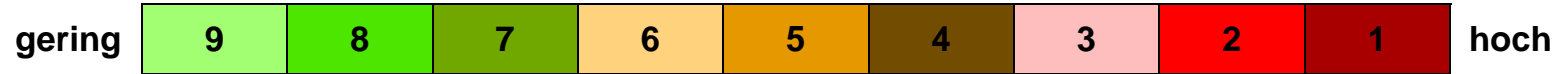

Abbildung 117: Verknüpfungsmatrix (oben) der Vorkommenswahrscheinlichkeit des Schwarzstorchs mit der Summe der Nutzungswahrscheinlichkeiten aller Freizeitaktivitäten sowie Farbskala (unten) der Ausprägung des Konfliktpotenzials.

\section{Erläuterungen:}

Vorkommenswahrscheinlichkeit: Bezeichnet die Wahrscheinlichkeit, dass auf einer Fläche während des Sommers ein relevantes Vorkommen des Schwarzstorchs auftritt. Die Modellierung der Vorkommenswahrscheinlichkeit basiert auf der flächenbezogenen Besiedlungsbzw. Nachweisdichte von brütenden bzw. Nahrung suchenden Schwarzstorchen. Die Vorkommenswahrscheinlichkeit wurde in drei Klassen (hoch, mittel, gering) klassifiziert und kann als Maß für die Sensibilität der betreffenden Fläche gegenüber Störungseinflüssen durch die Indikator-Erholungsaktivitäten interpretiert werden.

Nutzungswahrscheinlichkeit: Fungiert als Maß für die modellierte Nutzungsintensität aller Indikator-Erholungsaktivitäten. Es werden drei linear skalierte Werteklassen (hoch, mittel, ge- 
ring) differenziert.Die Nutzungswahrscheinlichkeit ist als potenzielle Erholungsnachfrage auf Basis theoretischer Annahmen und von GIS-basierten Modellierungen zu verstehen.

Konfliktpotenzial-Ausprägung bzw. Konfliktpotenzialstufe: Aus der Verknüpfung von drei Skalenstufen der Vorkommenswahrscheinlichkeit mit drei Skalenstufen der Nutzungswahrscheinlichkeit ergeben sich im Fall des Schwarzstorchs neun unterschiedliche Konfliktpotenzialausprägungen. Jede Kombination von Vorkommens- und Nutzungswahrscheinlichkeit repräsentiert eine spezifische Konflikt(potenzial)situation, die wiederum jeweils spezifische Managementziele und spezifischen Managementbedarf erfordern kann. Unter "Managementbedarf" ist zu verstehen, dass auf Flächen unterschiedlicher Ausprägung von Konfliktpotenzialen jeweils unterschiedliche Maßnahmenoptionen in unterschiedlicher Kombination als besonders geeignet erscheinen können, um die vorrangigen Managementziele zu erreichen. Die Verknüpfungsmatrix erleichtert somit einerseits die Zuordnung von prioritären Maßnahmenoptionen zu bestimmten Konfliktsituationen und andererseits über die Farbcodes der Karte in Abbildung 116 die näherungsweise räumliche Zuordnung zu entsprechenden Konflikträumen. 


\subsection{Generelle Ziele für das Konfliktmanagement in Bezug auf den Schwarzstorch}

Als eine prioritäre Zielart des Natur- und Artenschutzes (Anhang I der Vogelschutzrichtlinie) ergeben sich generelle Ziele in Bezug auf die Indikatorart Schwarzstorch im Biosphärenpark Wienerwald vorrangig aus naturschutzrechtlichen Schutzbestimmungen der Vogelschutzrichtlinie der EU sowie des Naturschutzrechtes beider betroffener Bundesländer. Aus der hohen Wertigkeit des Schwarzstorchs als Schutzgut des Naturschutzes lässt sich in weiterer Folge die Notwendigkeit ableiten, Nutzungsinteressen unterschiedlicher Landnutzergruppen den Artenschutzzielen unterzuordnen.

Im Hinblick auf die Steuerung von Konfliktpotenzialen zwischen Freizeit- und Erholungsnutzungen einerseits und den Zielen und Interessen betreffend den Schwarzstorch andererseits sind in der biosphärenparkweiten Betrachtung vor allem folgende übergeordnete Ziele maßgeblich:

- Schutz und Verbesserung des Erhaltungszustandes der Brutpopulation sowie des langfristigen Bruterfolges des Schwarzstorchs im Wienerwald

- Erhaltung und Verbesserung von Habitaten des Schwarzstorchs (Horststandorte, Bruthabitate, Nahrungsflächen) im Wienerwald

- Vermeidung und Minimierung von Störungen, die zu Verschlechterungen des Populationszustandes, des Bruterfolges und der Lebensräume des Schwarzstorchs im Wienerwald führen können 


\subsection{Schwarzstorch: Zuordnung von Leitfunktionen und Managementzielen zu Konfliktpotenzialstufen}

In der nachstehenden Tabelle 40 werden prioritäre Leitfunktionen und Zielausrichtungen für das Konfliktmanagement in Bezug auf die Indikatorart Schwarzstorch nach Ausprägungsformen des Konfliktpotenzials differenziert. Die Priorisierungen und Zuweisungen von Leitfunktionen und spezifischen Managementzielen spiegeln den unterschiedlichen Managementbedarf je nach Konfliktpotenzialstufe wider und sind als Orientierungshilfe für die nachfolgende Auswahl besonders geeigneter Managementoptionen zu verstehen.

Tabelle 40: Zuordnung von Leitfunktionen und Managementzielen zu Konfliktpotenzialausprägungen für den Schwarzstorch

\begin{tabular}{|c|c|c|c|c|}
\hline \multicolumn{5}{|c|}{$\begin{array}{l}\text { SCHWARZSTORCH: } \\
\text { Zuordnung von Leitfunktionen und Managementzielen zu Konfliktpotenzialausprägungen }\end{array}$} \\
\hline 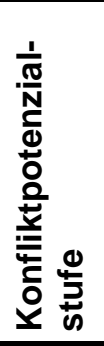 & 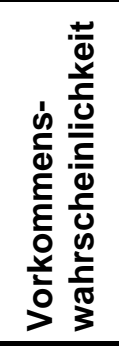 & 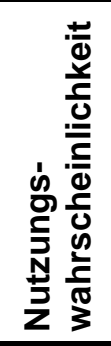 & Leitfunktion & Ziele \\
\hline 1 & hoch & hoch & $\begin{array}{l}\text { Habitatschutz } \\
\text { Schwarzstorch - Ex- } \\
\text { tensivierungsbereic } \\
\text { h (Störungsredukti- } \\
\text { on, Beruhigung) }\end{array}$ & $\begin{array}{l}\text { - Reduktion bzw. Minimierung von Störungen } \\
\text { (Horststandorte, Bruthabitate, Nahrungsflächen) infolge } \\
\text { von Freizeitbetrieb durch aktive Beruhigung von Brut- } \\
\text { und Nahrungshabitaten; Reduktion der Erholungsnach- } \\
\text { frage (März bis August) } \\
\text { - Reduktion bzW. Minimierung von Störungen durch be- } \\
\text { triebliche Aktivitäten von Jagd-, Forst- und Landwirt- } \\
\text { schaft } \\
\text { - Erhaltung und Verbesserung von Horststandorten, } \\
\text { Bruthabitaten und Nahrungshabitaten der Schwarz- } \\
\text { storchpopulation zur Sicherung des Bruterfolges }\end{array}$ \\
\hline 2 & hoch & mittel & $\begin{array}{l}\text { Habitatschutz } \\
\text { Schwarzstorch - Ex- } \\
\text { tensivierungsbereic } \\
\text { h (Störungsredukti- } \\
\text { on, Beruhigung) }\end{array}$ & $\begin{array}{l}\text { - Reduktion bzw. Minimierung von Störungen } \\
\text { (Horststandorte, Bruthabitate, Nahrungsflächen) infolge } \\
\text { von Freizeitbetrieb durch aktive Beruhigung von Brut- } \\
\text { und Nahrungshabitaten; Reduktion der Erholungsnach- } \\
\text { frage (März bis August) } \\
\text { - Reduktion bzw. Minimierung von Störungen durch be- } \\
\text { triebliche Aktivitäten von Jagd-, Forst- und Landwirt- } \\
\text { schaft } \\
\text { - Erhaltung und Verbesserung von Horststandorten, } \\
\text { Bruthabitaten und Nahrungshabitaten der Schwarz- } \\
\text { storchpopulation zur Sicherung des Bruterfolges }\end{array}$ \\
\hline 3 & mittel & hoch & $\begin{array}{l}\text { Habitatschutz } \\
\text { Schwarzstorch - Ex- } \\
\text { tensivierungsbereic } \\
\text { h (Störungsredukti- } \\
\text { on, Beruhigung) }\end{array}$ & $\begin{array}{l}\text { Reduktion von Störungen (Horststandorte, Bruthabitate, } \\
\text { Nahrungsflächen) infolge von Freizeitbetrieb, um das } \\
\text { vorhandene Lebensraumpotenzial für den Schwarz- } \\
\text { storch besser ausschöpfen zu können; aktive Beruhi- } \\
\text { gung von Horststandorten und Nahrungshabitaten } \\
\text { durch verbesserte und wirksamere Besucherlenkung } \\
\text { (März bis August) } \\
\text { - Reduktion von Störungen durch betriebliche Aktivitäten } \\
\text { von Jagd-, Forst- und Landwirtschaft }\end{array}$ \\
\hline
\end{tabular}




\begin{tabular}{|c|c|c|c|c|}
\hline 4 & hoch & gering & $\begin{array}{l}\text { Habitatschutz } \\
\text { Schwarzstorch - } \\
\text { Aufrecherhaltung } \\
\text { geringer Störungs- } \\
\text { intensität (Ruhig } \\
\text { halten) }\end{array}$ & $\begin{array}{l}\text { - Vermeiden zusätzlicher Störungen (Horststandorte, } \\
\text { Nahrungsflächen) infolge von Freizeitbetrieb; Ruhighal- } \\
\text { ten von Brut- und Nahrungshabitaten; Geringhalten der } \\
\text { Erholungsnachfrage (insb. März bis August) } \\
\text { - Ausweitung des Flächentyps durch gezielte Lenkungs- } \\
\text { maßnahmen } \\
\text { - Vermeiden bzw. Minimierung von Störungen durch be- } \\
\text { triebliche Aktivitäten von Jagd-, Forst- und Landwirt- } \\
\text { schaft } \\
\text { - Erhaltung und Verbesserung von Horststandorten, } \\
\text { Bruthabitaten und Nahrungshabitaten der Schwarz- } \\
\text { storchpopulation zur Sicherung des Bruterfolges } \\
\end{array}$ \\
\hline 5 & mittel & mittel & $\begin{array}{l}\text { Prüfbereich Mana- } \\
\text { gementbedarf - } \\
\text { Habitatverbesserun } \\
\text { g und/oder Stö- } \\
\text { rungsreduktion }\end{array}$ & $\begin{array}{l}\text { - Förderung des Schwarzstorchs } \\
\text { - Teilraumbezogene Beurteilung des Managementbe- } \\
\text { darfs und situationsabhängige Priorisierung von Hand- } \\
\text { lungsstrategien je nach der Aufwand-Nutzen-Relation: } \\
\text { - } \quad \text { Reduktion von Störungen durch Freizeitbetrieb (Ex- } \\
\text { tensivierung) bei hoher Habitatqualität und realisti- } \\
\text { schen Umsetzungs- und Erfolgschancen von Besu- } \\
\text { cherlenkungsmaßnahmen } \\
\text { - Gezielte Maßnahmen zur Habitatverbesserung bei } \\
\text { vorhandenem Lebensraumpotenzial und entspre- } \\
\text { chenden Umsetzungsmöglichkeiten } \\
\text { - Flexible Kombination von Elementen beider Hand- } \\
\text { lungsstrategien } \\
\text { - Keine Managementeingriffe (,business-as-usual“) } \\
\text { bzw. Beibehaltung des Status quo bei Nichtvorliegen } \\
\text { oben genannter Voraussetzungen }\end{array}$ \\
\hline 6 & gering & hoch & $\begin{array}{l}\text { Eignungsbereich für } \\
\text { kleinräumige Kom- } \\
\text { pensations- bzw. } \\
\text { Alternativangebote } \\
\text { zur Besucherlen- } \\
\text { kung }\end{array}$ & $\begin{array}{l}\text { - Kanalisierung bereits in hoher Dichte und flächig vor- } \\
\text { handener (sowie teils unerlaubter) Freizeitaktivitäten } \\
\text { durch Schaffung kleinräumiger bzw. punktueller alterna- } \\
\text { tiver Freizeitangebote } \\
\text { - Sicherung und Erhaltung von derzeit noch störungsar- } \\
\text { men, ökologisch sensiblen Bereichen in anderen Teilen } \\
\text { des Biosphärenparks durch kleinräumige Attraktivierung } \\
\text { in den betreffenden, gering sensiblen Teilräumen } \\
\text { - Verbesserung der Kommunizierbarkeit und Akzeptanz } \\
\text { von Nutzungsrestriktionen in anderen, sensiblen Teil- } \\
\text { räumen durch Bereitstellung legaler Kompensationsan- } \\
\text { gebote }\end{array}$ \\
\hline 7 & mittel & gering & $\begin{array}{l}\text { Erhaltung geringer } \\
\text { Störungsintensität } \\
\text { (Beruhight halten) - } \\
\text { Habitatverbesserun } \\
\text { g Schwarzstorch }\end{array}$ & $\begin{array}{l}\text { - Vermeiden der Zunahme von Störungen } \\
\text { (Horststandorte, Bruthabitate, Nahrungsflächen) infolge } \\
\text { von Freizeitbetrieb; Ruhig Halten von Brut- und Nah- } \\
\text { rungshabitaten; Gering Halten der Erholungsnachfrage } \\
\text { (insb. März bis August) } \\
\text { - Verbesserung des Lebensraumpotenzials für den } \\
\text { Schwarzstorch durch gezielte Maßnahmen des } \\
\text { Habitatmanagements } \\
\text { - Vermeiden bzw. Minimierung von Störungen durch be- } \\
\text { triebliche Aktivitäten von Jagd-, Forst- und Landwirt- } \\
\text { schaft während der Anwesenheitszeit des Schwarz- } \\
\text { storchs (März - August) }\end{array}$ \\
\hline
\end{tabular}




\begin{tabular}{|c|c|c|c|c|}
\hline 8 & gering & mittel & $\begin{array}{l}\text { Kein Schwarz- } \\
\text { storch-spezifischer } \\
\text { Managementbedarf } \\
\text { (,bussiness-as- } \\
\text { usual“-Bereich) - } \\
\text { Vorbehaltsbereich } \\
\text { für kleinräumige } \\
\text { Attraktivierungsang } \\
\text { ebote zur Besu- } \\
\text { cherlenkung }\end{array}$ & $\begin{array}{l}\text { - Beibehaltung Status quo (,business-as-usual“-Bereich), } \\
\text { weil derzeit keine besonderen Managementziele aus } \\
\text { der Indikatorart Schwarzstorch ableitbar sind } \\
\text { - Optionsbereich für kleinräumige } \\
\text { Attraktivierungsmaßnahmen } \\
\text { - Vorbehaltsbereich für relevante Ziele, die sich gegebe- } \\
\text { nenfalls aus Managementbedarf in Bezug auf andere } \\
\text { Indikatorarten ableiten lassen können }\end{array}$ \\
\hline 9 & gering & gering & $\begin{array}{l}\text { Erhaltung Status } \\
\text { quo - Kein } \\
\text { Schwarzstorch- } \\
\text { spezifischer Mana- } \\
\text { gementbedarf }\end{array}$ & $\begin{array}{l}\text { - Beibehaltung Status quo („,business-as-usual“-Bereich), } \\
\text { weil derzeit kein besonderer Managementbedarf aus } \\
\text { der Indikatorart Schwarzstorch ableitbar } \\
\text { - Vorrang für relevante Ziele, die sich gegebenenfalls aus } \\
\text { Managementbedarf in Bezug auf andere Indikatorarten } \\
\text { ableiten lassen können } \\
\text { - Beibehaltung derzeit extensiver Freizeitnutzungen im } \\
\text { Sinne genereller Zielsetzungen des Biosphärenparks zu } \\
\text { naturraumverträglichem Freizeitbetrieb (Ruhig Halten) }\end{array}$ \\
\hline
\end{tabular}




\subsection{Schwarzstorch: Übersicht über Handlungsoptionen des Besuchermanage- ments und querschnittsorientierte Maßnahmenoptionen nach Konfliktpoten- zialstufen}

In der nachstehenden Tabelle 41 werden die in Kap. 4.2 beschriebenen Maßnahmenoptionen aus den Bereichen Besuchermanagement und querschnittsorientierte Maßnahmen den Konfliktpotenzialstufen in Bezug auf den Schwarzstorch zugeordnet. Die Nummerierung der Maßnahmen entspricht der Gliederung in Kap. 4.2. Die Zordnung gibt Hinweise darauf, welche Maßnahmen grundsätzlich besonders geeignet erscheinen, um in unterschiedlichen Konfliktpotenzialsituationen zum Konfliktmanagement beizutragen. Die unterschiedlichen Konfliktpotenzialstufen entsprechen den farblich differenzierten Konfliktpotenzialräumen in Abbildung 116. Die Tabelle ist als Entscheidungshilfe für die Maßnahmenauswahl und priorisierung zu verstehen und sollte im Sinne eines Maßnahmenbaukastens benutzt werden. 
Tabelle 41: Besonders geeignete Maßnahmenoptionen (Besuchermanagement, Querschnittsmaßnahmen) je Konfliktpotenzialstufe für den Schwarzstorch

\section{SCHWARZSTORCH}

Zuordnung von Maßnahmenoptionen (Besuchermanagement, Querschnittsmaßnahmen) zu den Konfliktpotenzialausprägungen

\begin{tabular}{|c|c|c|c|c|c|c|c|c|c|c|}
\hline \multicolumn{2}{|c|}{ Konfliktpotenzialstufe } & 1 & 2 & 3 & 4 & 5 & 6 & 7 & 8 & 9 \\
\hline & Vorkommenswahrscheinlichkeit (Sensibilität) & hoch & hoch & mittel & hoch & mittel & gering & mittel & gering & gering \\
\hline & Nutzungswahrscheinlichkeit (Erholungsnachfrage) & hoch & mittel & hoch & gering & mittel & hoch & gering & mittel & gering \\
\hline \multicolumn{11}{|c|}{ Maßnahmenoptionen } \\
\hline Nr.* & Bezeichnung & & & & & & & & & \\
\hline \multicolumn{11}{|c|}{ Besuchermanagement - Freizeit- und Erholungsaktivitäten } \\
\hline \multicolumn{11}{|c|}{ Kleinräumige Maßnahmenoptionen } \\
\hline $\boldsymbol{A}$ & Räumlich-zeitliche Nutzungsrestriktionen & & & & & & & & & \\
\hline A1 & $\begin{array}{l}\text { Vereinheitlichung rechtlich verankerter Nutzungsverbote und -gebote (Kern- } \\
\text { zonen) }\end{array}$ & $\mathbf{X}$ & $\mathbf{x}$ & $\mathbf{x}$ & $\mathbf{X}$ & $\mathbf{X}$ & $\mathbf{X}$ & $\mathbf{x}$ & $\mathbf{x}$ & $\mathbf{x}$ \\
\hline $\mathrm{A} 2$ & Verzicht auf Ausbau der Wegeinfrastruktur & $\mathbf{X}$ & $\mathbf{x}$ & $\mathbf{X}$ & $\mathbf{x}$ & $\mathrm{X} ?$ & & $\mathbf{x}$ & $(\mathrm{X})$ & $\mathbf{x}$ \\
\hline A3 & Verlegung bzw. Auflassung von Wegen und kernzonengerechtes Wegenetz & $\mathbf{X}$ & $\mathrm{X}$ & $(\mathrm{X})$ & $\mathbf{x}$ & $\mathrm{X} ?$ & & (X) & & \\
\hline A4 & $\begin{array}{l}\text { Freiwillige Vereinbarungen zu örtlich begrenzten, räumlichen und/oder zeitli- } \\
\text { chen Nutzungseinschränkungen }\end{array}$ & $\mathbf{X}$ & $\mathbf{x}$ & $(\mathrm{X})$ & $\mathbf{X}$ & $X ?$ & & $(\mathrm{X})$ & & \\
\hline A5 & $\begin{array}{l}\text { Jagdrechtlich begründete Nutzungsrestriktionen (Wildschutzgebiete, befriste- } \\
\text { te jagdliche Sperrgebiete) }\end{array}$ & & & & & & & & & \\
\hline$B$ & Besucherlenkung durch De-Attraktivierung & & & & & & & & & \\
\hline B1 & Landschaftsgestalterische Maßnahmen zur Verringerung der Zugänglichkeit & $\mathbf{X}$ & $\mathbf{X}$ & $\mathbf{X}$ & $\mathbf{X}$ & $\mathbf{X} ?$ & & $\mathbf{X}$ & & \\
\hline B2 & Verzicht auf Schneeräumung & & & & & & & & & \\
\hline B3 & Management von Infrastruktur mit Schleusenfunktion & $\mathbf{x}$ & $\mathrm{X}$ & $\mathbf{x}$ & $\mathbf{x}$ & $\mathrm{X} ?$ & & $\mathbf{x}$ & & \\
\hline C & $\begin{array}{l}\text { Kleinräumige Kompensations- bzw. Alternativangebote zur Besucher- } \\
\text { lenkung }\end{array}$ & & & & & & & & & \\
\hline C1 & Hundeauslaufbereiche & & & & & & $\mathbf{x}$ & & $\mathrm{X} ?$ & \\
\hline $\mathrm{C} 2$ & Night Trails für Jogger & & & & & & $\mathbf{X}$ & & $X ?$ & \\
\hline $\mathrm{C} 3$ & Night Trails und Winter Trails für Mountainbiker & & & & & & $\mathbf{X}$ & & $\mathrm{X} ?$ & \\
\hline $\mathrm{C} 4$ & Mountainbike Technik Trails & & & & & & $\mathbf{X}$ & & $\mathrm{X} ?$ & \\
\hline $\mathrm{C5}$ & Mountainbike Downhill-Strecken & & & & & & $\mathbf{X}$ & & $\mathrm{X} ?$ & \\
\hline C6 & Betreute Picknickplätze & & & & & & $\mathbf{x}$ & & $\mathrm{X} ?$ & \\
\hline $\mathrm{C} 7$ & Wildlife Viewing-Einrichtungen (Schwarzstorch-Beobachungswarten) & & $\mathbf{x}$ & $(\mathrm{X})$ & & & & & & \\
\hline
\end{tabular}




\begin{tabular}{|c|c|}
\hline C & $\begin{array}{l}\text { RZSTORCH } \\
\text { ng von Maßnahmenoptionen (Besuchermanagement, } C\end{array}$ \\
\hline Konfl & ktpotenzialstufe \\
\hline & Vorkommenswahrscheinlichkeit (Sensibilität) \\
\hline & Nutzungswahrscheinlichkeit (Erholungsnachfrage) \\
\hline$\overline{\mathrm{Maßn}}$ & ahmenoptionen \\
\hline $\mathrm{Nr}^{*}$ & Bezeichnung \\
\hline $\mathrm{C} 8$ & Verstärkte Instandhaltung und Pflege der bestehenden Wegeinfrastruktur \\
\hline$D$ & Verstärkte Information, Kontrolle und Vollzug in Schwerpunktbereichen \\
\hline D1 & Konsequente Durchsetzung des Wegegebotes in Kernzonen \\
\hline D2 & Verstärkte Information, Kontrolle und Sanktionierung in sensiblen Gebieten \\
\hline D3 & $\begin{array}{l}\text { Verstärkte Information, Kontrolle und Sanktionierung im Bereich von Kom- } \\
\text { pensations- und Alternativangeboten (siehe C) }\end{array}$ \\
\hline$E$ & Teilraumbezogene Beurteilung von Zielpriorität und Managementbedarf \\
\hline E1 & $\begin{array}{l}\text { Prüfung des spezifischen Managementbedarfs und situationsabhängige } \\
\text { Priorisierung von Handlungsstrategien und Maßnahmenoptionen (A, B, D) in } \\
\text { Bezug auf den Schwarzstorch oder andere Indikatorarten }\end{array}$ \\
\hline Quers & hnittsorientierte Handlungsoptionen und Begleitmaßnahmen \\
\hline $\boldsymbol{F}$ & Information, Kommunikation, Öffentlichkeitsarbeit \\
\hline F1 & Zielgruppenspezifische Kommunikation und Öffentlichkeitsarbeit \\
\hline F1.1 & Interne Kommunikation \\
\hline F1.2 & Aus- und Weiterbildung \\
\hline F1.3 & Kommerzielle Angebote \\
\hline F1.4 & Kommunikationsschleusen \\
\hline & $\begin{array}{l}\text { - Nutzung von punktuellem Schwarzstorch-Beobachungsangebot (C7) als } \\
\text { Kommunikationsschleuse }\end{array}$ \\
\hline & $\begin{array}{l}\text { - Nutzung von kleinräumigen Attraktivierungsangeboten }(C 1-C 6) \text { als Kom- } \\
\text { munikationsschleusen }\end{array}$ \\
\hline $\mathrm{F} 2$ & $\begin{array}{l}\text { Biosphärenparkweite und zielgruppenspezifische Konzepte für Kommunikati- } \\
\text { on und Öffentlichkeitsarbeit }\end{array}$ \\
\hline F3 & רsstrukturen mit institutionalisierten \\
\hline
\end{tabular}

\begin{tabular}{l||c|c|c|c||c|c|c||c||c|}
\hline & $\mathbf{1}$ & $\mathbf{2}$ & $\mathbf{3}$ & $\mathbf{4}$ & $\mathbf{5}$ & $\mathbf{6}$ & $\mathbf{7}$ & $\mathbf{8}$ & $\mathbf{9}$ \\
\hline & hoch & hoch & mittel & hoch & mittel & gering & mittel & gering & gering \\
\hline & hoch & mittel & hoch & gering & mittel & hoch & gering & mittel & gering \\
\hline
\end{tabular}

\begin{tabular}{|c|c|c|c|c|c|c|c|c|}
\hline & & & & & $\bar{X}$ & (X) & $\bar{x}$ & \\
\hline & & & & & & & & \\
\hline$X$ & $x$ & $x$ & $x$ & $X ?$ & & $X$ & & \\
\hline \multirow[t]{4}{*}{$x$} & $\mathrm{X}$ & $\mathbf{X}$ & $x$ & $X ?$ & & $x$ & & \\
\hline & & & & & $\mathrm{X!}$ & & $X ?$ & \\
\hline & & & & $\mathrm{X!}$ & & & $\mathrm{X!}$ & \\
\hline & & & & $X !$ & & & $\mathrm{X!}$ & \\
\hline \multicolumn{9}{|c|}{$\begin{array}{c}\text { Geeignete und kohärente Integration von relevanten Inhalten, um die Erreichung der je-- } \\
\text { weiligen Managementziele je nach spezifischer Konfliktpotenzialausprägung und klein- } \\
\text { räumiger Maßnahmenauswahl zu unterstützen }\end{array}$} \\
\hline & $x$ & (X) & & & & & & \\
\hline & & & & & $x$ & & $X ?$ & \\
\hline \multicolumn{9}{|c|}{$\begin{array}{c}\text { Geeignete und kohärente Integration von relevanten Inhalten, um die Erreichung der je- } \\
\text { weiligen Managementziele je nach spezifischer Konfliktpotenzialausprägung und klein- } \\
\text { räumiger Maßnahmenauswahl zu unterstützen }\end{array}$} \\
\hline & & ärenpe & ite $A$ & dung & $\ln \mathrm{Je}$ & o Fall & hlen & \\
\hline
\end{tabular}




\begin{tabular}{|c|c|}
\hline & $\begin{array}{l}\text { RZSTORCH } \\
\text { ung von Maßnahmenoptionen (Besuchermanagement, Querschn }\end{array}$ \\
\hline Kon & ktpotenzialstufe \\
\hline & Vorkommenswahrscheinlichkeit (Sensibilität) \\
\hline & $\begin{array}{l}\text { Nutzungswahrscheinlichkeit (Erholungsnachfrage) } \\
\end{array}$ \\
\hline 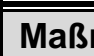 & ahmenoptionen \\
\hline $\mathrm{Nr}^{*}$ & Bezeichnung \\
\hline F4 & Zentrale Internet-Marketingplattform für naturbasierte Freizeitaktivitäten \\
\hline & - Kenntlichmachung von Habitatschutz- \& Ruhezonen mit "no go"-Charakter \\
\hline & $\begin{array}{l}\text { - Ausweisung von kleinräumigen Attraktivierungsangeboten für Freizeitnut- } \\
\text { zungen }(C 1-C 7)\end{array}$ \\
\hline F5 & Naturraumverträgliches Geocaching-Konzept \\
\hline & - Kenntlichmachung von Habitatschutz- \& Ruhezonen mit "no go"-Charakter \\
\hline F6 & Verbesserung der Beschilderung \\
\hline & $\begin{array}{l}\text { - wirksamere Information über räumlich-zeitliche Nutzungsrestriktionen (A1 - } \\
\text { A5) }\end{array}$ \\
\hline & - unter Verweis auf lokale Kompensationsangebote $(\mathrm{C} 1-\mathrm{C} 7)$ andernorts \\
\hline F7 & Digitale Geoinformationen für Wegeverwaltung und Routenplanung \\
\hline & - Kenntlichmachung von Habitatschutz- \& Ruhezonen mit "no go"-Charakter \\
\hline & $\begin{array}{l}\text { - Ausweisung von kleinräumigen Attraktivierungsangeboten für Freizeitnut- } \\
\text { zungen }(C 1-C 7)\end{array}$ \\
\hline F8 & Präsenz der Grundeigentümer \\
\hline F9 & Schulung von Aufsichts- und Kontrollorganen \\
\hline & - Kommunikations- und Konfliktmanagement \\
\hline & - Besondere Schulung betreffend Ökologie des Schwarzstorchs \\
\hline F10 & Fortbildungsangebot \\
\hline F11 & Positive Kommunikation von Restriktionen über (Kompensations-)Angebote \\
\hline F12 & Verzicht auf Maßnahmen zur Bewerbung und zum Freizeitmarketing \\
\hline
\end{tabular}

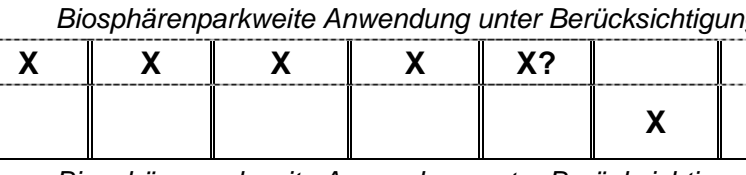

Xer Konfliktpotenziale

Biosphärenparkweite Anwendung unter Berücksichtigung der Konfliktpotenziale

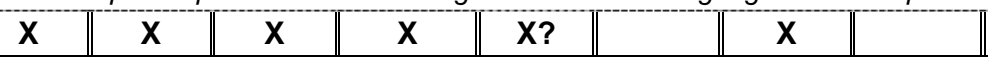
Biosphärenparkweite Anwendung unter Berücksichtigung der unterschiedlichen Konfliktpotenziale

\begin{tabular}{|c|c|c|c|c|c|c|c|}
\hline$x$ & $x$ & $x$ & $x$ & $X ?$ & & (X) & \\
\hline $\mathrm{X}$ & $X$ & $x$ & $\mathrm{X}$ & $X ?$ & $\mathrm{X}$ & (X) & $X ?$ \\
\hline
\end{tabular}

Biosphärenparkweite Anwendung unter Berücksichtigung der Konfliktpotenziale
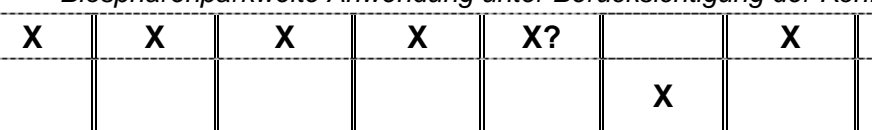

$X ?$

Biosphärenparkweite Anwendung unter Berücksichtigung der Konfliktpotenziale Biosphärenparkweite Anwendung unter Berücksichtigung der Konfliktpotenziale Biosphärenparkweite Anwendung unter Berücksichtigung der Konfliktpotenziale \begin{tabular}{c|c|c|c|c|}
$\mathbf{X}$ & $\mathbf{X}$ & $\mathbf{X}$ & $\mathbf{X}$ & $\mathbf{X} ?$ \\
Geeignete Integration von Schwarzstorch-relevanten Inhalten in Fortbildungs- und Kurs- \\
angebote für Freizeitnutzergruppen
\end{tabular}

Biosphärenparkweite Anwendung in allen relevanten Kommunikationsmaßnahmen

\begin{tabular}{l||l||l||l||l||l||c||c||}
$\mathbf{X}$ & $\mathbf{X}$ & $\mathbf{X}$ & $\mathbf{X}$ & $\mathbf{X}$ & $\mathbf{X}$ & & $\mathbf{X}$
\end{tabular}




\begin{tabular}{|c|c|c|c|c|c|c|c|c|c|c|}
\hline \multicolumn{11}{|c|}{$\begin{array}{l}\text { SCHWARZSTORCH } \\
\text { Zuordnung von Maßn }\end{array}$} \\
\hline \multicolumn{2}{|c|}{ Konfliktpotenzialstufe } & 1 & $\overline{2}$ & $\overline{3}$ & $\overline{4}$ & $\overline{5}$ & 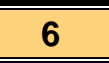 & $\overline{7}$ & $\overline{8}$ & $\overline{9}$ \\
\hline & Vorkommenswahrscheinlichkeit (Sensibilität) & hoch & hoch & mittel & hoch & mittel & gering & mittel & gering & gering \\
\hline & Nutzungswahrscheinlichkeit (Erholungsnachfrage) & hoch & mittel & hoch & gering & mittel & hoch & gering & mittel & gering \\
\hline \multicolumn{11}{|c|}{ Maßnahmenoptionen } \\
\hline Nr. ${ }^{*}$ & Bezeichnung & & & & & & & & & \\
\hline G & Kooperative Konfliktlösungsstrategien & & & & & & & & & \\
\hline \multicolumn{2}{|c|}{ G1-G4 (Details siehe Kap. 4.1.3.2.2) } & $\mathbf{x}$ & $\mathbf{x}$ & $\mathbf{x}$ & $\mathrm{x}$ & $\mathrm{X} ?$ & & $(\mathrm{X})$ & & \\
\hline & $\begin{array}{l}\text { - zur Aushandlung freiwilliger Vereinbarungen zu örtlich begrenzten, räumli- } \\
\text { chen und/oder zeitlichen Nutzungsrestriktionen für den Freizeitbetrieb (A1 - } \\
\text { A5) }\end{array}$ & $\mathrm{x}$ & $\mathbf{x}$ & $\mathrm{x}$ & $\mathrm{x}$ & $\mathrm{X} ?$ & & $(\mathrm{X})$ & & \\
\hline & $\begin{array}{l}\text { - zur Standortfindung und Entwicklung kleinräumiger Kompensations- bzw. } \\
\text { Alternativangebote für Freizeitaktivitäten (C1 - C7) }\end{array}$ & & & & & & $\mathrm{x}$ & & $\mathrm{X} ?$ & \\
\hline & $\begin{array}{l}\text { - zur Vereinbarung von Maßnahmen, die verstärkte sektorübergreifende Ko- } \\
\text { ordination zwischen Landnutzergruppen erfordern }\end{array}$ & $\mathrm{x}$ & $\mathrm{x}$ & $\mathrm{x}$ & $\mathrm{x}$ & $\mathrm{X} ?$ & $x$ & $(\mathrm{X})$ & $\mathrm{X} ?$ & \\
\hline $\mathrm{H}$ & Kontrolle und Vollzug von Verhaltensregeln & \multirow{2}{*}{\multicolumn{9}{|c|}{$\begin{array}{l}\text { Biosphärenparkweite Anwendung wird empfohlen (zu Schwerpunktbereichen für Kontrolle } \\
\text { und Vollzug siehe D) }\end{array}$}} \\
\hline \multicolumn{2}{|c|}{$\mathrm{H} 1-\mathrm{H} 2$ (Details siehe Kap. 4.1.3.2.2) } & & & & & & & & & \\
\hline \begin{tabular}{|l|l|l}
$I$ \\
11
\end{tabular} & Biosphärenparkweite Verhaltensregeln & \multirow{2}{*}{\multicolumn{9}{|c|}{$\begin{array}{c}\text { Biosphärenparkweite Anwendung wird unabhängig von der jeweiligen Konfliktpotenzial- } \\
\text { ausprägung empfohlen }\end{array}$}} \\
\hline 11 & Einheitliche Rahmen-Nutzungsregeln im Biosphärenpark & & & & & & & & & \\
\hline $\mathbf{J}$ & $\begin{array}{l}\text { Maßnahmenauswahl in Abhängigkeit vom Ergebnis der Prüfung des } \\
\text { spezifischen Managementbedarfs (vgl. E1) }\end{array}$ & & & & & $\mathrm{X} !$ & & & $\mathrm{X} !$ & \\
\hline $\mathrm{J} 1$ & $\begin{array}{l}\text { Flexible und geeignete Ausgestaltung von querschnittsorientierten Maßnah- } \\
\text { men in Abhängigkeit vom Prüfergebnis zum Managementbedarf und der hie- } \\
\text { raus abgeleiteten kleinräumigen Maßnahmenauswahl (vgl. E1) }\end{array}$ & & & & & $\mathrm{X} !$ & & & $\mathrm{X} !$ & \\
\hline \multicolumn{11}{|c|}{ Generelle Maßnahmen für weitere Landnutzergruppen } \\
\hline $\mathrm{K}$ & Raumplanung, Infrastrukturplanung & & & & & & & & & \\
\hline K1 & Forcierte Umsetzung von Zielen einer nachhaltigen Siedlungsentwicklung & & & & & & & & & \\
\hline & $\begin{array}{l}\text { - Verhinderung von Habitatverlusten und siedlungsinduzierten Störungen } \\
\text { insb. von Nahrungshabitaten durch Vermeidung von Baulandwidmungen } \\
\text { auf relevanten Grünlandflächen, konsequente Rückwidmungen von dies- } \\
\text { bezüglichen Baulandreserven sowie Einhaltung von Pufferzonen zu } \\
\text { Schwarzstorchhabitaten }\end{array}$ & $\mathbf{X !}$ & $\mathrm{X} !$ & $\mathbf{X !}$ & $\mathrm{X!}$ & $\mathrm{x}$ & & $\mathrm{x}$ & $\mathrm{x}$ & $\mathrm{x}$ \\
\hline
\end{tabular}




\begin{tabular}{|c|c|c|c|c|c|c|c|c|c|c|}
\hline \multirow{2}{*}{\multicolumn{2}{|c|}{$\begin{array}{l}\text { SCHWARZSTORCH } \\
\text { Zuordnung von Maßnahmenoptionen (Besuchermanagement, Querschnit } \\
\text { Konfliktpotenzialstufe }\end{array}$}} & & & & & & & & & \\
\hline & & $\overline{1 .}$ & $\overline{2}$ & $\overline{3}$ & $\overline{4}$ & $\overline{5}$ & 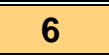 & $\overline{7}$ & $\overline{8}$ & $\overline{9}$ \\
\hline \multicolumn{2}{|r|}{$\begin{array}{l}\text { Vorkommenswahrscheinlichkeit (Sensibilität) } \\
\end{array}$} & hoch & hoch & mittel & hoch & mittel & gering & mittel & gering & gering \\
\hline \multicolumn{2}{|r|}{ Nutzungswahrscheinlichkeit (Erholungsnachfrage) } & hoch & mittel & hoch & gering & mittel & hoch & gering & mittel & gering \\
\hline \multicolumn{11}{|c|}{ Maßnahmenoptionen } \\
\hline \multicolumn{2}{|c|}{\begin{tabular}{l|l} 
Nr. $^{*}$ & Bezeichnung \\
\end{tabular}} & & & & & & & & & \\
\hline \multirow[t]{2}{*}{$\mathrm{K} 2$} & $\begin{array}{l}\text { Verstärkte Sicherung von störungsarmen, ökologisch bedeutsamen Frei- und } \\
\text { Grünräumen sowie von ökologischen Korridoren und Migrationsachsen }\end{array}$ & & & & & & & & & \\
\hline & $\begin{array}{l}\text { - Sicherung von Lebensräumen des Schwarzstorchs unter Berücksichtigung } \\
\text { des Lebensraumpotenzials und ausreichender Pufferzonen durch geeigne- } \\
\text { te überörtliche Festlegungen mit entsprechenden Widmungs- und Nut- } \\
\text { zungsverboten für die örtliche Raumplanung }\end{array}$ & $\mathrm{X} !$ & $\mathrm{X} !$ & $\mathrm{X} !$ & $\mathrm{X!}$ & $\mathrm{X}$ ? & & $\mathrm{x}$ & & \\
\hline K3 & $\begin{array}{l}\text { Errichtung und raumplanerische Absicherung von ökologischen } \\
\text { Querungshilfen für Wildtiere }\end{array}$ & & & & & & & & & \\
\hline K4 & $\begin{array}{l}\text { Verstärkte Prüfung von Entwicklungsvorhaben im Rahmen der Anwendung } \\
\text { von Prüfinstrumenten (UVP, SUP, NVP) }\end{array}$ & $\mathrm{x} !$ & $\mathrm{X} !$ & $\mathrm{X} !$ & $\mathrm{X} !$ & $x$ & & $\mathrm{x}$ & & $(\mathrm{X})$ \\
\hline K5 & $\begin{array}{l}\text { Schaffung eines Flächenpools für Ausgleichsmaßnahmen und Aktivierung } \\
\text { geeigneter Flächen zur Kompensation von Eingriffen in den Naturraum }\end{array}$ & & & & & & $\mathrm{X} !$ & & $\mathrm{x}$ & $(\mathrm{X})$ \\
\hline K6 & $\begin{array}{l}\text { Aktive und koordinierende Rolle der Raumordnung bei der Standortplanung } \\
\text { und Raumverträglichkeitsprüfung von neuen bzw. alternativen Angeboten für } \\
\text { Freizeitaktivitäten (C) }\end{array}$ & & & & & & $\mathrm{X!}$ & & $\mathrm{X}$ ? & \\
\hline \multirow{2}{*}{$\begin{array}{ll}L \\
L 1\end{array}$} & Sektorübergreifende Kooperation aller Landnutzungsbereiche & & & & & & & & & \\
\hline & $\begin{array}{l}\text { Verbesserte und abgestimmte Öffentlichkeitsarbeit, Bewusstseinsbildung, } \\
\text { Kommunikation und Information }\end{array}$ & \multicolumn{9}{|c|}{$\begin{array}{c}\begin{array}{c}\text { Biosphärenparkweite Anwendung unter Berücksichtigung der unterschiedlichen Konflikt- } \\
\text { potenzialausprägungen }\end{array} \\
\text {. }\end{array}$} \\
\hline L2 & $\begin{array}{l}\text { Verstärkte Mitarbeit aller Nutzergruppen bei der Ausarbeitung des Manage- } \\
\text { mentplans für den Biosphärenpark Wienerwald }\end{array}$ & \multicolumn{9}{|c|}{$\begin{array}{c}\begin{array}{c}\text { Biosphärenparkweite Anwendung unter Berücksichtigung der unterschiedlichen Konflikt- } \\
\text { potenzialausprägungen }\end{array}\end{array}$} \\
\hline L3 & $\begin{array}{l}\text { Aktive Beteiligung und Einbeziehung von Landnutzerinteressen in in } \\
\text { partizipative Prozesse zur Entwicklung von kleinräumigen Angebotsschaffun- } \\
\text { gen zur Besucherlenkung }\end{array}$ & & & & & & $\mathrm{x}$ & & $\mathbf{X}$ ? & \\
\hline \multicolumn{11}{|c|}{ Monitoring und adaptives Management } \\
\hline$M$ & Besuchermonitoring & & & & & & & & & \\
\hline M1 & $\begin{array}{l}\text { Besuchermonitoring mit Hilfe anonymisierter Bewegungsdaten von Handy- } \\
\text { nutzerlnnen }\end{array}$ & $x$ & $x$ & $x$ & $\mathrm{x}$ & $x$ & $(X)$ & $x$ & $(X)$ & $(X)$ \\
\hline
\end{tabular}




\begin{tabular}{|c|c|c|c|c|c|c|c|c|c|c|}
\hline \multirow{2}{*}{\multicolumn{2}{|c|}{$\begin{array}{l}\text { SCHWARZSTORCH } \\
\text { Zuordnung von Maßnahmenoptionen (Besuchermanagement, Querschni } \\
\text { Konfliktpotenzialstufe } \\
\end{array}$}} & & & & nfl & & 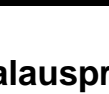 & & & \\
\hline & & 1 & 2 & 3 & 4 & 5 & 6 & 7 & 8 & 9 \\
\hline & Vorkommenswahrscheinlichkeit (Sensibilität) & hoch & hoch & mittel & hoch & mittel & gering & mittel & gering & gering \\
\hline & Nutzungswahrscheinlichkeit (Erholungsnachfrage) & hoch & mittel & hoch & gering & mittel & hoch & gering & mittel & gering \\
\hline \multicolumn{11}{|c|}{ Maßnahmenoptionen } \\
\hline $\mathrm{Nr}^{*}$ & Bezeichnung & & & & & & & & & \\
\hline M2 & Beobachtungen und Zählungen von Besucherströmen & $\mathbf{X}$ & $\bar{x}$ & $\bar{x}$ & $\bar{x}$ & $\bar{x}$ & $\bar{x}$ & $\bar{x}$ & $\bar{x}$ & $\bar{x}$ \\
\hline M3 & Befragungen & \multicolumn{9}{|c|}{$\begin{array}{l}\text { Durchführung wird biosphärenparkweit empfohlen; Befragungsdesign und Auswertung } \\
\text { sollten unterschiedliche Konfliktpotenziale und entsprechende Managementoptionen be- } \\
\text { rücksichtigen }\end{array}$} \\
\hline$N$ & Erfolgskontrolle zu vereinbarten Nutzungsregeln & & & & & & & & & \\
\hline N1 & Monitoring der Wirksamkeit von getroffenen Nutzungsvereinbarungen & $\mathbf{x}$ & $\mathbf{x}$ & $\mathbf{x}$ & $\mathbf{x}$ & $\mathrm{X} ?$ & $\mathbf{x}$ & & $\mathrm{X} ?$ & \\
\hline
\end{tabular}

Zeichenerklärung: $\quad \mathbf{X}$... prioritäre Managementoption; $\mathbf{X}$ ! ... stark prioritäre Managementoption; $(\mathbf{X})$... mit Vorbehalt geeignete bzw. weniger prioritäre Managementoption; $\mathbf{X}$ ? ... Eignung der Managementoption von spezifischer Prüfung des Maßnahmenbedarfs abhängig 


\subsection{Spezifische Maßnahmenoptionen für weitere Landnutzergruppen in Bezug auf den Schwarzstorch}

\section{Naturschutz}

Ziele:

- Erhaltung von Horststandorten, Brut- und Nahrungshabitaten durch Sicherung der Brutpopulation des Schwarzstorchs und des Bruterfolges

- Verbesserung von Habitaten des Schwarzstorchs und des Lebensraumpotenzials

\section{Handlungsstrategie:}

- Erstellung von Schutzkonzept und Managementplan zur Erhaltung und zur Verbesserung von Schwarzstorchhabitaten

- Prüfung und Entwicklung von Umsetzungsmechanismen

- Aktives Einbringen von Artenschutzinteressen in Vorhaben zur Besucherlenkung

\section{Maßnahmenoptionen:}

Erstellung eines Schutzkonzeptes und eines Managementplans zur Sicherung von aktuellen Horststandorten, Brut- und Nahrungshabitaten des Schwarzstorchs: Für aktuell vom Schwarzstorch besiedelte bzw. genutzte Lebensräume sollten unter Leitung und in der koordinativen Kompetenz des Naturschutzes ein naturschutzfachliches Schutzkonzept und ein Managementplan erstellt werden, die folgende Komponenten beinhalten sollten (vgl. Frank \& Berg, 2001):

o Einrichtung von Horstschutzzonen im Umkreis von bis zu 300 m von Horststandorten mit geeigneten Managementmaßnahmen (vgl. Frank \& Berg, 2001)

o Kartierung und Erhaltung von Bruthabitaten des Schwarzstorchs, einschließlich von potenziellen Horststandorten, mit geeigneten Managementmaßnahmen (vgl. Frank \& Berg, 2001)

o Kartierung, Erhaltung und Verbesserung von Nahrungshabitaten, insb. Fließgewässer mit ausreichendem Sichtschutz durch bachbegleitende Gehölzstreifen, Teiche und Tümpel, Feuchtwiesen und andere extensive Wiesenflächen sowie feuchte Waldstandorte

o Sektor- und Nutzergruppen übergreifende Koordination von Managementmaßnahmen durch Naturschutzorgane

Erstellung eines Managementplans zur Habitatverbesserung und zur Förderung des Lebensraumpotenzials für den Schwarzstorch: Die aktuelle Verbreitung des Schwarzstorchs zur Reproduktionszeit im Wienerwald ist bereits durch bestehende Habitatveränderungen sowie durch bestehende Störungen infolge von Freizeitbetrieb geprägt. Dies bedeutet, dass das Lebensraumpotenzial des Schwarzstorchs im Wienerwald größer ist als das aktuelle Vorkommensgebiet. Insbesondere in denjenigen Teilräumen, die derzeit als Randbereich des Verbreitungsgebietes erscheinen und mittlere Nachweisdichten aufweisen, ist davon auszugehen, dass der Lebensraum durch gezielte Habitatmanagement- und Besucherlenkungsmaßnahmen mit vergleichsweise geringem Aufwand effektiv ausgeweitet und so zur Bestands- und Arterhaltung beigetragen werden kann. Ein Managementplan zur Habitatverbesserung sollte insbesondere folgende Faktoren berücksichtigen: 
- Naturschutzfachliche Bewertung von limitierenden Lebensraumfaktoren und effektiven Verbesserungsmöglichkeiten

o Maßnahmen zur Förderung von Horststandorten

- Verbesserung von potenziellen Bruthabitaten des Schwarzstorchs, einschließlich von potenziellen Horststandorten, mit geeigneten Managementmaßnahmen (vgl. Frank \& Berg, 2001)

o Verbesserung von potenziellen Nahrungshabitaten, insb. Fließgewässer mit ausreichendem Sichtschutz durch bachbegleitende Gehölzstreifen, Teiche und Tümpel, Feuchtwiesen und andere extensive Wiesenflächen sowie feuchte Waldstandorte

o Sektor- und Nutzergruppen übergreifende Koordination von Habitatverbesserungsmaßnahmen durch Naturschutzorgane

Umsetzung von Managementmaßnahmen zum Habitatschutz und zur Habitatverbesserung im Rahmen der Kernzonen-Managementpläne, von Vertragsnaturschutzmodellen oder von freiwilligen Vereinbarungen mit Grundeigentümern: Die Umsetzung von Maßnahmen sowohl zur Erhaltung bestehender Habitate als auch zur Förderung des Lebensraumpotenzials außerhalb des bestehenden Kernverbreitungsgebietes sollte innerhalb von Kernzonen in der Verantwortung des Biosphärenparkmanagements durch Verankerung in den Kernzonen-Managementplänen erfolgen. Da die Kernzonen als Naturschutz- bzw. Landschaftsschutzgebiete ausgewiesen sind, ist dies als Aufgabenbereich des hoheitlichen Naturschutzes zu betrachten. Außerhalb von Kernzonen sollten geeignete Vertragsnaturschutzmodelle oder anderweitige Vereinbarungen mit Grundeigentümern geprüft, entwickelt und angewendet werden.

\section{Forstwirtschaft}

Ziele:

- Vermeiden von Störungen von Schwarzstorchhabitaten durch betriebliche Tätigkeiten

- Erhaltung von Horststandorten und Bruthabitaten

- Managementmaßnahmen zur Habitatverbesserung

Handlungsstrategie:

- Verzicht auf forstliche Eingriffe in Horstschutzbereichen

- Artenschutzdienliche Waldbewirtschaftung und gezieltes Waldmanagement zur Habitatverbesserung

- Aktives Einbringen von forstlichen Interessen in Vorhaben zur Besucherlenkung

Maßnahmenoptionen:

Vermeidung von Störungen durch forstbetriebliche Tätigkeiten: In Bruthabitaten sollten Störungen des Brutbetriebs durch forstliche Arbeiten während der Anwesenheitszeit des Schwarzstrochs (März - August) minimiert und weitestmöglich vermieden werden. Dringende forstliche Arbeiten (z. B. Forstschutzmaßnahmen) sollten möglichst außerhalb der Brutzeit durchgeführt werden.

Erhaltung von Horstbäumen und Verzicht auf forstliche Eingriffe im unmittelbaren Umfeld von Horststandorten: Soweit es sich nicht um Kernzonen des Biosphärenparks 
handelt, in denen ein generelles forstliches Bewirtschaftungsverbot besteht, sollte im Bereich von Horststandorten auf forstliche Nutzungseingriffe verzichtet werden.

Abstimmung der Waldbewirtschaftung in Bruthabitaten auf Schutzziele: Das Waldmanagement in Bruthabitaten sollte schutzzieldienlich erfolgen, d.h. ganzjährig auf die Erhaltung und Verbesserung günstiger Habitatbedingungen für den Schwarzstorch ausgerichtet sein. Waldbehandlungsmaßnahmen sollten mit dem Naturschutz abgestimmt sein, z. B. im Rahmen eines naturschutzfachlichen Managementplans für den Schwarzstorch.

Naturschutzfachlich abgestimmte Maßnahmen zur Habitatverbesserung und zur Förderung des Lebensraumpotenzials: Die Forstwirtschaft kann auch in Waldbeständen, die derzeit vom Schwarzstorch nur in geringer bis mittlerer Dichte besiedelt sind, durch vergleichsweise wenig aufwandsintensive Maßnahmen zu einer wirksamen Verbesserung des Lebensraumpotenzials beitragen. Potenziell geeignete Maßnahmen zur Habitatverbesserung in Brut- und Nahrungshabitaten umfassen z. B. (vgl. Frank \& Berg, 2001):

o Leichte Durchforstung in Horstnähe zur Verbesserung der Durchfliegbarkeit

- Erhöhung des Totholzanteils

o Erhaltung von horstgeeigneten Altbaumbeständen

o Verzicht auf forstliche Bewirtschaftung ufernaher Flächen und Förderung von naturnahen bachbegleitenden Gehölzen

o Erhaltung von feuchten Waldtypen und Buchenaltbeständen mit feuchtem Innenklima als Nahrungshabitat

o Verzicht auf Ausbau von Forstwegen

\section{Landwirtschaft}

Ziele:

- Vermeiden von Störungen von Nahrungshabitaten des Schwarzstorchs durch betriebliche Tätigkeiten

- Erhaltung von Nahrungshabitaten

- Managementmaßnahmen zur Habitatverbesserung

Handlungsstrategie:

- Vermeiden von Habitatverlusten und -verschlechterungen

- Artenschutzdienliche Bewirtschaftung von Grünlandstandorten und gezielte Maßnahmen zur Habitatverbesserung

- Aktives Einbringen von landwirtschaftlichen Interessen in Vorhaben zur Besucherlenkung

Maßnahmenoptionen:

Vermeidung von Störungen durch landwirtschaftliche Tätigkeiten auf Nahrungsflächen: Zur Anwesenheitszeit des Schwarzstorchs (März - August) bzw. zu Tageszeiten der Nahrungssuche während dieser Jahreszeit sollten Störungen durch betriebliche Tätigkeiten und landwirtschaftliche Bewirtschaftungsmaßnahmen auf Nahrungsflächen (feuch- 
te und extensiv genutzte Wiesen) des Schwarzstorchs minimiert bzw. weitest möglich vermieden werden.

Erhaltung von Nahrungsflächen: Feuchte bzw. extensiv genutzte Wiesen, die dem Schwarzstorch als Nahrungsfläche dienen, sind als Schlüsselhabitate unbedingt zu erhalten. Dies beinhaltet insbesondere die Vermeidung von Flächennutzungsänderungen (Ackerung, Bebauung), Drainagierungen oder Intensivierungen der Grünlandbewirtschaftung. Die Erhaltung von Nahrungshabitaten kann im Rahmen geeigneter ÖPULMaßnahmen, anderer Vertragsnaturschutzmodelle oder durch anderweitige freiwillige Rücksichtnahme seitens der Bewirtschafter erfolgen.

Abstimmung der Bewirtschaftung von Nahrungsflächen auf Schutzziele: Die Bewirtschaftung von Grünlandstandorten, die vom Schwarzstorch als Nahrungsflächen genutzt werden, sollte auf Erfordernisse des Artenschutzes abgestimmt werden. In Abstimmung mit dem Naturschutz kann dies z. B. in der zeitlichen Rücksichtnahme bei Bewirtschaftungsmaßnahmen bestehen, wie der Abstimmung von Mahdterminen und Schnitthäufigkeit auf die Anwesenheitszeit und die tageszeitliche Nahrungssucherhythmik des Schwarzstorchs.

Naturschutzfachlich abgestimmte Maßnahmen zur Habitatverbesserung und zur Förderung des Lebensraumpotenzials von Nahrungsflächen: Aktive landwirtschaftliche Maßnahmen zur Lebensraumverbesserung für den Schwarzstorch sollten mit dem Naturschutz abgestimmt sein und können z. B. umfassen:

o Revitalisierung (Wiedervernässung) von drainagierten Feuchtwiesen

- Extensivierung von derzeit intensiv genutzten Grünlandflächen

o Belassen von gewässerbegleitenden Ufergehölzen

o Einrichtung von nicht bewirtschafteten Pufferstreifen in Ufernähe

\section{Jagdwirtschaft}

Ziele:

- Vermeiden von Störungen von Schwarzstorchhabitaten durch die Jagdausübung

- Erhaltung von Schwarzstorchhabitaten

- Unterstützung von Managementmaßnahmen zur Habitatverbesserung

Handlungsstrategie:

- Selbstbeschränkung jagdlicher Aktivitäten in Habitaten des Schwarzstorchs

- Verzicht auf Errichtung von Reviereinrichtungen

- Aktives Einbringen von jagdlichen Interessen in Vorhaben zur Besucherlenkung

Maßnahmenoptionen:

Vermeiden von Störungen durch Selbsteinschränkung jagdlicher Tätigkeiten: Im Bereich von Horststandorten, in Brut- sowie in Nahrungshabitaten sollten jagdliche Aktivitäten, die zu einer Störung des natürlichen Verhaltens des Schwarzstorchs und zu einer Beeinträchtigung des Bruterfolgs führen können, zur Anwesenheitszeit des Scharzstorchs (März - August) weitest möglich vermieden bzw. minimiert werden. Dies kann beispielsweise durch entsprechende Vorgaben in Jagdpachtverträgen, durch die freiwillige Aus- 
weisung von Horstschutzzonen in Jagdkonzepten sowie durch die räumliche und zeitliche Berücksichtigung in Bejagungsstrategien erfolgen.

Rücksichtnahme bei der Errichtung jagdbetrieblicher Reviereinrichtungen: In aktuell vom Schwarzstorch besiedelten Kernhabitaten (Horststandorte, Brut- und Nahrungshabitate) sollte auf die Errichtung von Reviereinrichtungen, wie Hochsitzen, Schussflächen, Fütterungen, etc., verzichtet werden, da diese Einrichtungen oft mit habitatverändernden Eingriffen und mit Störungen im Zuge der Jagdausübung verbunden sind.

Unterstützung von Habitatverbesserungsmaßnahmen durch Ausweitung der jagdlichen Zurückhaltung auf potenzielle Lebensräume: Das Gebot der jagdlichen Selbstbeschränkung und des Verzichts auf die Anlage von Reviereinrichtungen sollte auf diejenigen Flächen ausgeweitet werden, auf denen Managementmaßnahmen zur Habitatverbesserung und zur Förderung des Lebensraumpotenzials für den Schwarzstorch von Seiten des Naturschutzes und der Grundeigentümer durchgeführt werden.

\section{Alle Nutzergruppen}

Betreffend die aktive Beteiligung aller Landnutzergruppen an partizipativen Prozessen zur Entwicklung von kleinräumigen Kompensations- bzw. Alternativangeboten für Freizeitnutzergruppen (vgl. Kap. 4.2.2, Empfehlung L3) siehe die Übersichtsmatrix in Tabelle 41 (Kap. 4.3.2.1.5). 


\subsection{Schwarzstorch: Übersicht über spezifische Maßnahmenoptionen für weitere Landnutzergruppen nach Konfliktpotenzialstufen}

In der nachstehenden Tabelle 42 werden die im vorangehenden Kap. 4.3.2.1.6 beschriebenen Maßnahmenoptionen für einzelne Landnutzergruppen sowie die in Kap. 4.2.3 beschriebenen Maßnahmen zum Monitoring von Wildtierpopulationen und -lebensräumen den Konfliktpotenzialstufen in Bezug auf den Schwarzstorch zugeordnet. Die Zordnung gibt Hinweise darauf, welche Maßnahmen grundsätzlich besonders geeignet erscheinen, um in unterschiedlichen Konfliktpotenzialsituationen zum Konfliktmanagement beizutragen. Die unterschiedlichen Konfliktpotenzialstufen entsprechen den farblich differenzierten Konfliktpotenzialräumen in Abbildung 116. Die Tabelle ist als Entscheidungshilfe für die Maßnahmenauswahl und -priorisierung zu verstehen und sollte im Sinne eines Maßnahmenbaukastens benutzt werden. 
Tabelle 42: Besonders geeignete sektorale Maßnahmenoptionen je Konfliktpotenzialstufe für den Schwarzstorch

\begin{tabular}{|c|c|c|c|c|c|c|c|c|c|}
\hline \multicolumn{10}{|c|}{$\begin{array}{l}\text { SCHWARZSTORCH } \\
\text { Zuordnung von spezifischen Maßnahmenoptionen für Landnutzergruppen zu den Konfliktpotenzialausprägungen }\end{array}$} \\
\hline Konfliktpotenzialstufe & $\overline{1.1}$ & 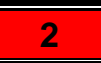 & 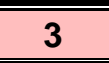 & 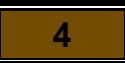 & 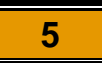 & 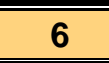 & $\overline{7}$ & 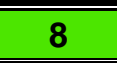 & 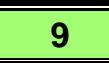 \\
\hline Vorkommenswahrscheinlichkeit (Sensibilität) & hoch & hoch & mittel & hoch & mittel & gering & mittel & gering & gering \\
\hline Nutzungswahrscheinlichkeit (Erholungsnachfrage) & hoch & mittel & hoch & gering & mittel & hoch & gering & mittel & gering \\
\hline \multicolumn{10}{|l|}{ Maßnahmenoptionen } \\
\hline \begin{tabular}{l|l|}
$\mathrm{Nr}^{*}$ & Bezeichnung \\
\end{tabular} & & & & & & & & & \\
\hline \multicolumn{10}{|l|}{ Weitere Landnutzergruppen } \\
\hline \multicolumn{10}{|l|}{ Naturschutz } \\
\hline $\begin{array}{l}\text { Schutzkonzept und Managementplan zur Sicherung und Erhaltung von Habitaten des } \\
\text { Schwarzstorchs (Horstschutzzonen, potenzielle Horststandorte, Bruthabitate, Nah- } \\
\text { rungshabitate) }\end{array}$ & $\mathrm{X} !$ & $\mathrm{X} !$ & $\mathrm{X} !$ & $\mathrm{X} !$ & $\mathrm{x}$ & & $\mathrm{x}$ & & \\
\hline $\begin{array}{l}\text { Habitatmanagementplan zur Verbesserung des Lebensraumpotenzials für den } \\
\text { Schwarzstorch (potenzielle Horststandorte, Brut- und Nahrungshabitate) }\end{array}$ & $(\mathrm{X})$ & $(\mathrm{X})$ & $\mathrm{X} !$ & $(\mathrm{X})$ & $\mathrm{X} ?$ & & $\mathrm{X} !$ & & \\
\hline $\begin{array}{l}\text { Umsetzung von Habitatschutz- und verbesserungsmaßnahmen im Rahmen von } \\
\text { Kernzonen-Managementplänen, Vertragsnaturschutzmodellen oder freiwilligen Ver- } \\
\text { einbarungen mit Grundeigentümern }\end{array}$ & $\mathrm{X} !$ & $\mathrm{X} !$ & $\mathrm{X} !$ & $\mathrm{X} !$ & $\mathrm{X} ?$ & & $\mathrm{X} !$ & & \\
\hline \multicolumn{10}{|l|}{ Forstwirtschaft } \\
\hline $\begin{array}{l}\text { Vermeidung von Störungen durch forstbetriebliche Tätigkeiten während der Anwe- } \\
\text { senheitszeit des Schwarzstorchs }\end{array}$ & $\mathrm{X} !$ & $\mathrm{X} !$ & $\mathrm{X} !$ & $\mathrm{X!}$ & $\mathrm{x}$ & & $\mathrm{x}$ & & \\
\hline $\begin{array}{l}\text { Erhaltung von Horstbäumen und Verzicht auf forstliche Eingriffe in deren unmittelba- } \\
\text { rem Umfeld }\end{array}$ & $\mathrm{X} !$ & $\mathrm{X} !$ & $\mathrm{X} !$ & $\mathrm{X} !$ & $\mathrm{x}$ & & $\mathrm{x}$ & & \\
\hline Schutzzieldienliche Waldbewirtschaftung in Bruthabitaten & $\mathrm{X} !$ & $\mathrm{X} !$ & $\mathbf{x}$ & $\mathrm{X} !$ & $\mathrm{X}$ & & $\mathbf{x}$ & & \\
\hline $\begin{array}{l}\text { Naturschutzfachlich abgestimmte Maßnahmen zur Habitatverbesserung und Förde- } \\
\text { rung des Lebensraumpotenzials (Verbesserung der Durchfliegbarkeit in Horstnähe, } \\
\text { Erhöhung Totholzanteil, Erhaltung von Altbaumbeständen, keine Bewirtschaftung } \\
\text { ufernaher Gehölzbestände, etc.) }\end{array}$ & (X) & (X) & $\mathrm{X} !$ & (X) & $\mathrm{X} ?$ & & $\mathrm{X} !$ & & \\
\hline \multicolumn{10}{|l|}{ Landwirtschaft } \\
\hline $\begin{array}{l}\text { Vermeiden von Störungen durch betriebliche Maßnahmen auf Nahrungsflächen zur } \\
\text { Anwesenheitszeit bzw. zu Tageszeiten der Nahrungssuche des Schwarzstorchs }\end{array}$ & $\mathrm{X} !$ & $\mathrm{X} !$ & $\mathrm{X!}$ & $\mathrm{X!}$ & $x$ & & $x$ & & \\
\hline $\begin{array}{l}\text { Erhaltung und Verbesserung von Nahrungsflächen des Schwarzstorchs im Rahmen } \\
\text { von ÖPUL-Maßnahmen, Vertragsnaturschutzmodellen oder freiwilliger Rücksicht- } \\
\text { nahme }\end{array}$ & $\mathrm{X} !$ & $\mathrm{X} !$ & $\mathrm{X} !$ & $\mathrm{X} !$ & $\mathrm{x}$ & & $\mathrm{X} !$ & & \\
\hline
\end{tabular}




\begin{tabular}{|c|c|c|c|c|c|c|c|c|c|c|}
\hline \multirow{2}{*}{\multicolumn{2}{|c|}{$\begin{array}{l}\text { SCHWARZSTORCH } \\
\text { Zuordnung von spezifischen Maßnahmenoptionen für Landnutzergruppe } \\
\text { Konfliktpotenzialstufe }\end{array}$}} & u & Ko। & & lat & & & & & \\
\hline & & $\overline{1}$ & 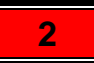 & 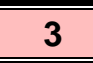 & 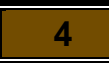 & 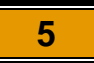 & 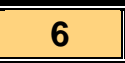 & $\overline{7}$ & 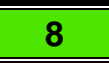 & $\overline{9}$ \\
\hline \multicolumn{2}{|r|}{\begin{tabular}{|ll} 
& Vorkommenswahrscheinlichkeit (Sensibilität) \\
\end{tabular}} & hoch & hoch & mittel & hoch & mittel & gering & mittel & gering & gering \\
\hline \multicolumn{2}{|r|}{$\begin{array}{ll}\text { Nutzungswahrscheinlichkeit (Erholungsnachfrage) } \\
\end{array}$} & hoch & mittel & hoch & gering & mittel & hoch & gering & mittel & gering \\
\hline \multicolumn{11}{|c|}{ Maßnahmenoptionen } \\
\hline $\mathrm{Nr}^{*}$ & Bezeichnung & & & & & & & & & \\
\hline \multicolumn{2}{|r|}{ Schutzzieldienliche Abstimmung der Bewirtschaftung von Nahrungsflächen } & $\mathrm{X!}$ & $\mathrm{X!}$ & $\mathrm{X!}$ & $\mathrm{X!}$ & $\mathbf{x}$ & & $\mathbf{x}$ & & \\
\hline \multicolumn{2}{|r|}{$\begin{array}{l}\text { Naturschutzfachlich abgestimmte Maßnahmen zur Habitatverbesserung und Förde- } \\
\text { rung des Lebensraumpotenzials außerhalb aktuell besiedelter Habitate (Revitalisie- } \\
\text { rung von Feuchtwiesen, Extensivierung intensiv genutzter Wiesenflächen, Pufferstrei- } \\
\text { fen in Gewässernähe, etc.) }\end{array}$} & $\mathrm{x}$ & $\mathrm{x}$ & $\mathrm{X} !$ & $\mathrm{x}$ & $\mathrm{X} ?$ & & $\mathrm{X} !$ & & \\
\hline \multicolumn{11}{|c|}{ Jagdwirtschaft } \\
\hline \multicolumn{2}{|r|}{$\begin{array}{l}\text { Selbsteinschränkung jagdliche Aktivitäten in Schwarzstorch-Habitaten } \\
\text { (Horststandorte, Brut- und Nahrungshabitate) zur Anwesenheitszeit des Schwarz- } \\
\text { storchs }\end{array}$} & $\mathrm{X} !$ & $\mathrm{X} !$ & $\mathrm{x}$ & $\mathrm{X} !$ & $\mathrm{x}$ & & $\mathrm{x}$ & & \\
\hline \multicolumn{2}{|r|}{$\begin{array}{l}\text { Verzicht auf Errichtung jagdbetrieblicher Einrichtungen bzw. zu deren Benützung } \\
\text { während der Anwesenheitszeit des Schwarzstorchs im Bereich von Schwarzstorch- } \\
\text { Habitaten }\end{array}$} & $\mathrm{X} !$ & $\mathrm{X} !$ & $\mathrm{x}$ & $\mathrm{X} !$ & $x$ & & $\mathrm{x}$ & & \\
\hline \multicolumn{2}{|r|}{$\begin{array}{l}\text { Ausgeweitete freiwillige Zurückhaltung bei der Jagdausübung und jagdbetrieblich be- } \\
\text { dingten Eingriffen in Bereichen, in denen Maßnahmen zur Verbesserung des Lebens- } \\
\text { raumpotenzials durchgeführt werden }\end{array}$} & (X) & (X) & $\mathrm{X} !$ & (X) & $\mathrm{x} ?$ & & $\mathrm{X} !$ & & \\
\hline \multicolumn{11}{|c|}{ Monitoring und adaptives Management } \\
\hline \multirow{2}{*}{\multicolumn{2}{|c|}{\begin{tabular}{l|l} 
Q & Erfolgskontrolle zu vereinbarten Nutzungsregeln \\
Q1 & Monitoring der Wirksamkeit von getroffenen Nutzungsvereinbarungen
\end{tabular}}} & & & & & & & & & \\
\hline & & $\mathbf{x}$ & $\mathbf{x}$ & $\mathbf{x}$ & $\mathbf{x}$ & $\mathrm{X} ?$ & $\mathbf{x}$ & & $\mathrm{X} ?$ & \\
\hline$R$ & Monitoring von Wildtierpopulationen und -lebensräumen & & & & & & & & & \\
\hline R1 & Einrichtung eines regelmäßigen Schwarzstorch-Monitorings & $\mathbf{x}$ & $\mathbf{X}$ & $\mathbf{x}$ & $\mathbf{X}$ & $\mathbf{x}$ & & $\mathbf{x}$ & & \\
\hline R2 & Fortführung des Ziesel-Monitorings & & & & & & & & & \\
\hline R3 & Monitoring von Schalenwildeinfluss auf die Waldvegetation & & & & & & & & & \\
\hline R4 & Monitoring von Wildtierbeständen, Wildabschüssen und Fallwild & & & & & & & & & \\
\hline R5 & Zusätzliches Monitoring spezifischer Wildtierindikatorarten & & & & & & & & & \\
\hline R6 & $\begin{array}{l}\text { Integration eines Wildtier-Monitorings in das allgemeine Biodiversitäts- } \\
\text { Monitoring }\end{array}$ & $\mathbf{x}$ & $\mathrm{x}$ & $\mathbf{x}$ & $\mathbf{x}$ & $\mathrm{x}$ & & $\mathbf{x}$ & & \\
\hline
\end{tabular}




\begin{tabular}{|c|c|c|c|c|c|c|c|c|c|c|}
\hline \multicolumn{11}{|c|}{$\begin{array}{l}\text { SCHWARZSTORCH } \\
\text { Zuordnung von spezif }\end{array}$} \\
\hline \multicolumn{2}{|c|}{ Konfliktpotenzialstufe } & 1 & $\overline{2}$ & $\overline{3}$ & $\overline{4}$ & $\overline{5}$ & $\overline{6}$ & $\overline{7}$ & $\overline{8}$ & 9 \\
\hline & Vorkommenswahrscheinlichkeit (Sensibilität) & hoch & hoch & $\overline{\text { mittel }}$ & hoch & $\overline{\text { mittel }}$ & gering & mittel & gering & gering \\
\hline & Nutzungswahrscheinlichkeit (Erholungsnachfrage) & hoch & mittel & hoch & gering & mittel & hoch & gering & mittel & gering \\
\hline \multicolumn{11}{|c|}{ Maßnahmenoptionen } \\
\hline $\mathrm{Nr}^{*}$ & Bezeichnung & & & & & & & & & \\
\hline R7 & Monitoringpflicht in Natura 2000-Gebieten & $\mathrm{X}$ & $\mathbf{x}$ & $\mathbf{x}$ & $\mathbf{x}$ & $\mathbf{x}$ & & $\mathbf{x}$ & & \\
\hline R8 & Monitoring der Wiesen & $\mathbf{x}$ & $\mathrm{x}$ & $\mathbf{x}$ & $\mathbf{x}$ & $\mathbf{x}$ & & $\mathbf{x}$ & & \\
\hline
\end{tabular}

Zeichenerklärung: $\quad \mathbf{X}$... prioritäre Managementoption; $\mathbf{X} !$... stark prioritäre Managementoption; $(\mathbf{X})$... mit Vorbehalt geeignete bzw. weniger prioritäre Managementoption; X? ... Eignung der Managementoption von spezifischer Prüfung des Maßnahmenbedarfs abhängig 


\subsubsection{Rothirsch}

Die Hinweise und Entscheidungshilfen dieses Kapitels 4.3.2.2 zu den Zielen und zur Maßnahmenauswahl für das Konfliktmanagement beziehen sich ausschließlich auf die Indikatorart Rothirsch. Es ist darauf hinzuweisen, dass jedes Konfliktmanagement in konkreten Teilräumen des Biosphärenparks stets auch Konfliktpotenziale und Managementbedarf in Bezug auf die anderen Indikatorarten berücksichtigen und auf deren Zusammenschau basieren sollte.

\subsection{Modellierung des Konfliktpotenzials im GIS}

Die nachfolgende Kartendarstellung (Abbildung 118) zeigt neun unterschiedliche Ausprägungsformen des Konfliktpotenzials zwischen der Indikatorart Rotwild und der Summe aller Indikator-Freizeitaktivitäten im Biosphärenpark Wienerwald. Das Konfliktpotenzial ergibt sich aus der Kombination der summierten Nutzungswahrscheinlichkeit aller Freizeitaktivitäten mit der Vorkommenswahrscheinlichkeit des Rotwilds.

Die Bezeichnung "-wahrscheinlichkeit“ im Zusammenhang mit Indikatorart und Indikatoraktivitäten wird verwendet, um auszudrücken, dass es sich um GIS-Modellierungen handelt, die neben empirischen Daten auch auf theoriegestützten Annahmen beruhen.

Für die Modellierung im GIS wurde die stufenlose Standardisierung der Konfliktpotenziale zwischen 0 und 1, wie sie in den Kartendarstellungen des Kapitels 3.4.3 vorgenommen wurde, auf eine diskrete Skala mit drei Werteklassen des Konfliktpotenzials umgerechnet. Hierfür wurde die Nutzungswahrscheinlichkeit der Indikatoraktivitäten linear in drei Werteabschnitte, d.h. in drei gleich große Skalenintervalle (hoch, mittel, gering), klassifiziert. Die Vorkommenswahrscheinlichkeit des Rotwilds beruht auf Angaben zur Verteilung und Lebensraumnutzung von Regionalexperten; die Klassifikation in drei Werteklassen (hoch, mittel, gering) folgt grundsätzlich der Abstufung, wie sie bei der Erstellung der Verbreitungskarte in Kap. 3.4.2.1(Abbildung 83) verwendet wurde.

Durch die Kombination von drei Klassen der Nutzungswahrscheinlichkeit mit drei Klassen der Vorkommenswahrscheinlichkeit des Rotwilds ergeben sich insgesamt neun Kombinationsmöglichkeiten, die unterschiedlichen Ausprägungsformen des Konfliktpotenzials entsprechen. Jeder der neun Konfliktpotenzialausprägungen wird ein eigener Farbwert zugewiesen. In denjenigen Bereichen des Biosphärenparks, wo das Rotwild aufgrund bestehender forstpolitischer Zielsetzungen nicht geduldet wird, besteht hiernach auch kein Konfliktpotenzial mit Freizeitaktivitäten; diese „rotwildfreien“ Bereiche sind in der Karte weiß dargestellt. Die Kombination erfolgt über die Verknüpfungsmatrix in Abbildung 119 mittels der darin festgelegten Verknüpfungsregeln.

Die Karte in der nachstehenden Abbildung 118 zeigt die Lage und räumliche Verteilung der neun Konfliktpotenzialstufen im Biosphärenpark. In den nachfolgenden Kapiteln werden den unter-schiedlichen Konfliktpotenzialausprägungen differenzierte Managementziele sowie besonders geeignete Maßnahmenoptionen für das Besuchermanagement und für weitere spezifische Landnutzergruppen zugeordnet. Über die Karte in Abbildung 118 ist näherungsweise die räumliche Zuordnung von vorrangigen Zielen und Maßnahmenoptionen des Konfliktmanagements zu Konflikträumen unterschiedlicher Konfliktpotenzialstufe möglich. 


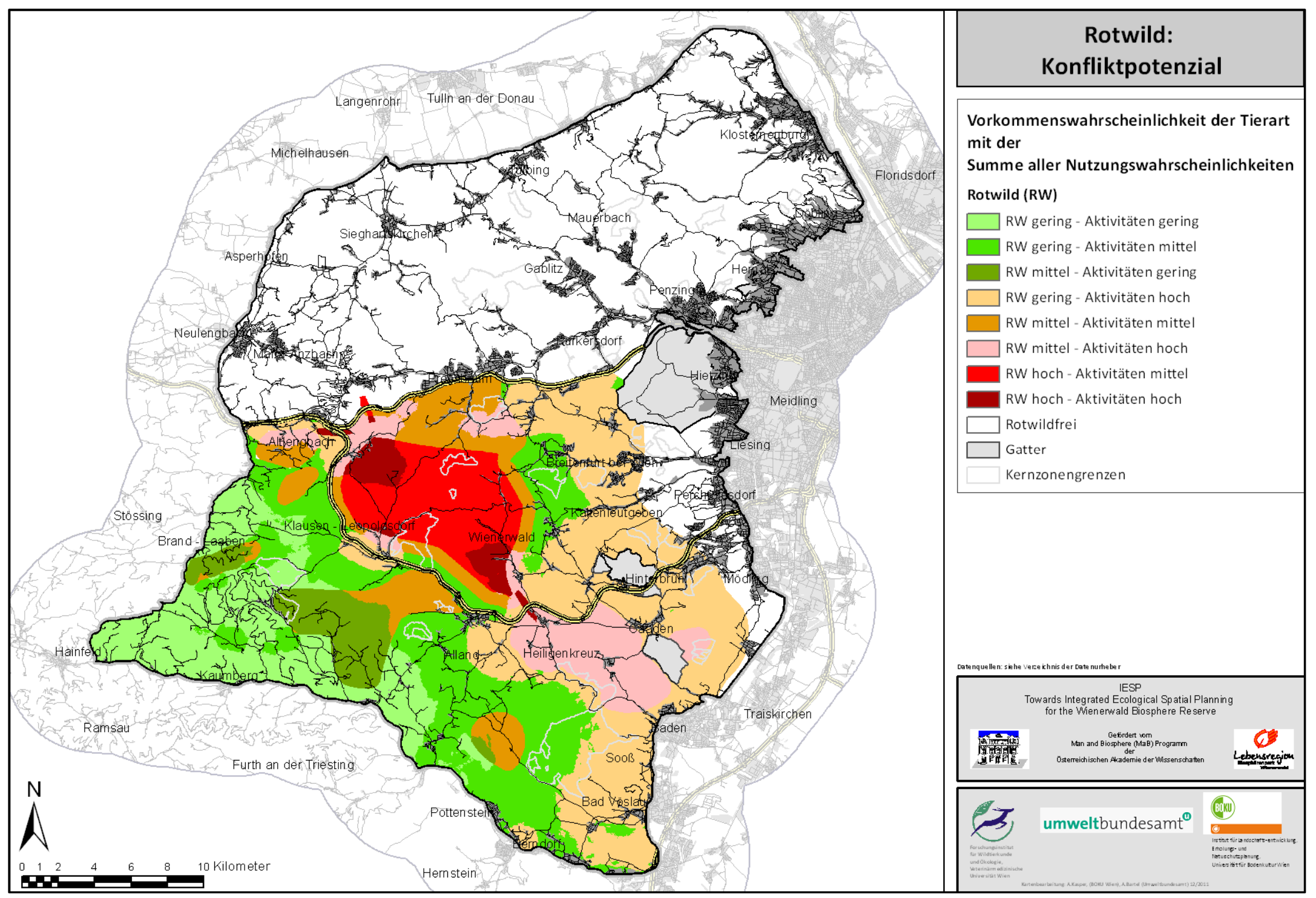

Abbildung 118: Räumliche Verteilung des Konfliktpotenzials zwischen dem Rothirsch und der Summe aller Freizeitaktivitäten in neun Werteklassen. 


\subsection{Verknüpfungsmatrix}

Mittels nachstehender Abbildung 119 wird die Vorkommenswahrscheinlichkeit des Rotwilds mit der summierten Nutzungswahrscheinlichkeit aller Indikator-Freizeitaktivitäten verknüpft. Über die aus der Abbildung ersichtlichen Vernüpfungsregeln ergeben sich neun Kombinationsmöglichkeiten, die unterschiedlichen Ausprägungsformen bzw. Stufen des Konfliktpotenzials entsprechen. Die Verknüpfungsmatrix erleichtert die Zuordnung von Zielen für das Konfliktmanagement (siehe Kap. 4.3.2.2.4) sowie von besonders geeigneten Maßnahmenoptionen (siehe Kap. 4.3.2.2.5 und Kap. 4.3.2.2.7) zu unterschiedlichen Konflikt(potenzial)situationen. Über die zugewiesenen Farbwerte wird weiters die näherungsweise räumliche Zuordnung von prioritären Managementoptionen in der Karte in Abbildung 118 (Kap. 4.3.2.2.1) möglich.

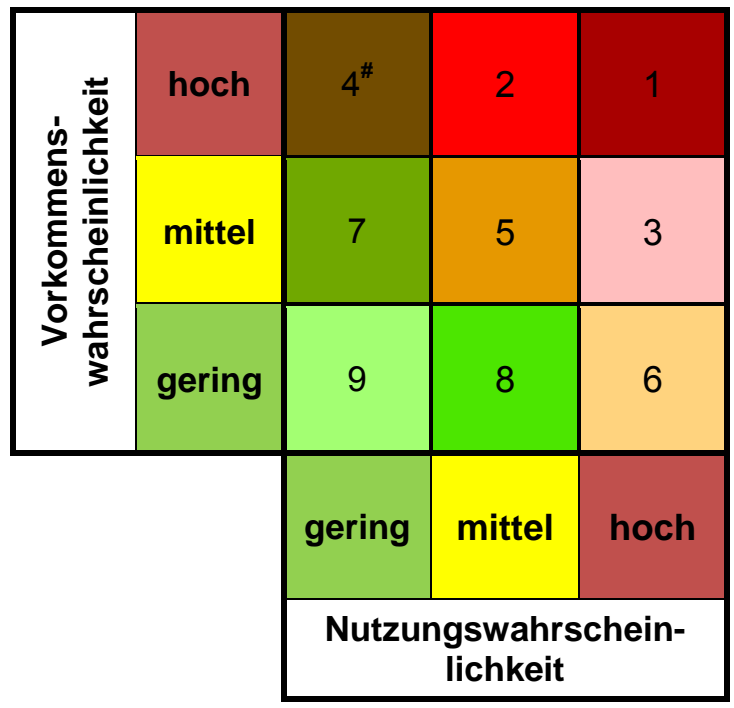

\section{Ausprägung Konfliktpotenzial}

\begin{tabular}{l|l|l|l|l|l|l|l|l|l|l|}
\hline gering & $0^{*}$ & 9 & 8 & 7 & 6 & 5 & $4^{\#}$ & 3 & 2 & 1 \\
\hline
\end{tabular}

Anmerkungen: * 0 ... rotwildfreies Gebiet, kein Konflikpotenzial; * Stufe 4 tritt in GIS-Modellierung nicht auf und fehlt daher in der Karte in Abbildung 118.

Abbildung 119: Verknüpfungsmatrix (oben) der Vorkommenswahrscheinlichkeit des Rotwilds mit der Summe der Nutzungswahrscheinlichkeiten aller Freizeitaktivitäten sowie Farbskala (unten) der Ausprägung des Konfliktpotenzials

\section{Erläuterungen:}

Vorkommenswahrscheinlichkeit: Bezeichnet die Wahrscheinlichkeit, dass auf einer Fläche Rotwild in höherer oder geringerer Bestandesdichte auftritt, d.h. die wahrscheinliche Vorkommensdichte des Rotwilds. Die räumliche Darstellung der Vorkommenswahrscheinlichkeit basiert auf dem lokalen Wissen von Regionalexperten zu Kerngebieten, randlichen Vorkommensgebieten, Wintereinständen und Wechseln des Rotwildes im Wienerwald; die betreffenden Angaben wurden durch den Vergleich mit langjährigen Abschusszahlen auf ihre Plausibilität überprüft. Die Vorkommenswahrscheinlichkeit wurde in drei Klassen (hoch, mittel, gering) klassifiziert und kann als Maß für die Sensibilität 
der betreffenden Fläche gegenüber Störungseinflüssen durch die IndikatorErholungsaktivitäten interpretiert werden.

Nutzungswahrscheinlichkeit: Fungiert als Maß für die modellierte Nutzungsintensität aller Indikator-Erholungsaktivitäten. Es werden drei linear skalierte Werteklassen (hoch, mittel, gering) differenziert.Die Nutzungswahrscheinlichkeit ist als potenzielle Erholungsnachfrage auf Basis theoretischer Annahmen und von GIS-basierten Modellierungen zu verstehen.

Konfliktpotenzial-Ausprägung bzw. Konfliktpotenzialstufe: Aus der Verknüpfung von drei Skalenstufen der Vorkommenswahrscheinlichkeit mit drei Skalenstufen der Nutzungswahrscheinlichkeit ergeben sich im Fall des Rotwilds neun unterschiedliche Konfliktpotenzialausprägungen. Jede Kombination von Vorkommens- und Nutzungswahrscheinlichkeit repräsentiert eine spezifische Konflikt(potenzial)situation, die wiederum jeweils spezifische Managementziele und spezifischen Managementbedarf erfordern kann. Unter "Managementbedarf“ ist zu verstehen, dass auf Flächen unterschiedlicher Ausprägung von Konfliktpotenzialen jeweils unterschiedliche Maßnahmenoptionen in unterschiedlicher Kombination als besonders geeignet erscheinen können, um die vorrangigen Managementziele zu erreichen. Die Verknüpfungsmatrix erleichtert somit einerseits die Zuordnung von prioritären Maßnahmenoptionen zu bestimmten Konfliktsituationen und andererseits über die Farbcodes der Karte in Abbildung 118 die näherungsweise räumliche Zuordnung zu entsprechenden Konflikträumen. 


\subsection{Generelle Ziele für das Konfliktmanagement in Bezug auf den Rothirsch}

Generelle Ziele in Bezug auf die Indikatorart Rotwild im Biosphärenpark Wienerwald lassen sich zum einen aus übergeordneten Nachhaltigkeitszielen zur Erhaltung und nachhaltigen Nutzung der biologischen Vielfalt und wildlebender Ressourcen ableiten, zum anderen aus rechtlichen Grundlagen, wie dem Jagdrecht, dem Naturschutzrecht und dem Biosphärenpark Wienerwald Gesetz der beiden Bundesländer Niederösterreich und Wien, dem Forstrecht, betrieblichen Zielen von Forst- und Jagdbetrieben bzw. Grundeigentümern sowie aus den Interessen von Jagdausübungsberechtigten.

Im Hinblick auf die Steuerung von Konfliktpotenzialen zwischen Freizeit- und Erholungsnutzungen einerseits und den Zielen und Interessen betreffend das Rotwild andererseits sind in der biosphärenparkweiten Betrachtung vor allem folgende übergeordnete Ziele besonders relevant:

- Erhaltung der Lebensräume und Populationen des Rotwilds in zumindest der derzeitigen Verbreitung

o Anmerkung: Der nördliche Teil des Biosphärenparks nördlich der Autobahntrasse der A1 ist gegenwärtig infolge forstwirtschaftlicher Zielsetzungen der Grundeigentümer als rotwildfrei zu betrachten, d.h. die Anwesenheit von Rotwild ist nicht grundsätzlich auszuschließen, gilt aber als unerwünscht.

- Vermeidung von Wildschäden durch Rotwild am Wald

- Erhaltung der nachhaltigen jagdlichen Nutzbarkeit der Rotwildbestände sowie des Jagdwerts der betreffenden Jagdreviere

- Erhaltung störungsarmer Kerngebiete, Einstandshabitate und Äsungsflächen des Rotwildes, insbesondere im Winter und im Frühjahr

- Vermeidung von Störungen, die zu Verschlechterungen für Lebensräume und Populationszustand sowie zur Beeinträchtigung der nachhaltigen Bejagbarkeit und Abschusserfüllung führen

- Gewährleistung der Lebensraumvernetzung und des Populationsaustauschs des Rotwilds innerhalb der Wienerwaldregion sowie auf überregionaler Ebene

o Anmerkung: Die Zielerreichung hängt von zwei wesentlichen Rahmenbedingungen ab. Dies ist zum einen die Errichtung von technischen Wildquerungshilfen für das Rotwild entlang der bestehenden Autobahntrassen A1 und A21. Zum anderen wäre ein Abrücken von der derzeitigen Grundeigentümerpolitik der Nicht-Duldung des Rotwildes im gesamten Bereich nördlich der A1 erforderlich. 


\subsection{Rothirsch: Zuordnung von Leitfunktionen und Managementzielen zu Kon- fliktpotenzialstufen}

In der nachstehenden Tabelle 43 werden prioritäre Leitfunktionen und Zielausrichtungen für das Konfliktmanagement in Bezug auf die Indikatorart Rothirsch nach Ausprägungsformen des Konfliktpotenzials differenziert. Die Priorisierungen und Zuweisungen von Leitfunktionen und spezifischen Managementzielen spiegeln den unterschiedlichen Managementbedarf je nach Konfliktpotenzialstufe wider und sind als Orientierungshilfe für die nachfolgende Auswahl besonders geeigneter Managementoptionen zu verstehen.

Tabelle 43: Zuordnung von Leitfunktionen und Managementzielen zu Konfliktpotenzialausprägungen für den Rothirsch

\begin{tabular}{|l||l||l|l|}
\hline \multicolumn{2}{|l|}{ ROTHIRSCH: } \\
Zuordnung von Leitfunktionen und Managementzielen zu Konfliktpotenzialausprägungen \\
\hline \hline
\end{tabular}




\begin{tabular}{|c|c|c|c|c|}
\hline 3 & mittel & hoch & $\begin{array}{l}\text { Kleinräumiges Kon- } \\
\text { fliktmanagement }\end{array}$ & $\begin{array}{l}\text { - Kleinräumige, flexible Maßnahmenabstimmung zwi- } \\
\text { schen Besuchermanagement und Bejagungsmanage- } \\
\text { ment auf Basis einer spezifischen Prüfung von Konflikt- } \\
\text { situation und Managementbedarf, um: } \\
\text { - störungsarme Kerngebiete, Einstände und Äsungsflä- } \\
\text { chen des Rotwilds durch Kanalisierung von Erho- } \\
\text { lungstätigkeiten zu erhalten } \\
\text { - Wildschäden am Wald zu vermeiden } \\
\text { - effiziente Bejagbarkeit, Abschusserfüllung und siche- } \\
\text { re Schussabgabe zu ermöglichen } \\
\text { - Wildtierpassagen und Korridore zu beruhigen }\end{array}$ \\
\hline $4^{*}$ & hoch & gering & $\begin{array}{l}\text { Ruhezone Rotwild - } \\
\text { Erhaltung geringer } \\
\text { Störungsintensität } \\
\text { (beruhigt halten) }\end{array}$ & $\begin{array}{l}\text { - Einrichtung von Ruhezonen für das Rotwild, auch au- } \\
\text { Berhalb von Kernzonen } \\
\text { - Geringhalten der Erholungsnachfrage durch Besucher- } \\
\text { lenkungsmaßnahmen, mit Präferenz für „sanfte“ Maß- } \\
\text { nahmen und Anreizen für Besucher in wenig sensiblen } \\
\text { Gebieten andernorts } \\
\end{array}$ \\
\hline 5 & mittel & mittel & $\begin{array}{l}\text { Kleinräumiges Kon- } \\
\text { fliktmanagement }\end{array}$ & $\begin{array}{l}\text { - Kleinräumige, flexible Maßnahmenabstimmung zwi- } \\
\text { schen Besuchermanagement und Bejagungsmanage- } \\
\text { ment auf Basis einer spezifischen Prüfung von Konflikt- } \\
\text { situation und Managementbedarf, um: } \\
\text { - störungsarme Kerngebiete, Einstände und Äsungsflä- } \\
\text { chen des Rotwilds durch Kanalisierung von Erho- } \\
\text { lungstätigkeiten zu erhalten } \\
\text { - Wildschäden amWald zu vermeiden } \\
\text { - effiziente Bejagbarkeit, Abschusserfüllung und siche- } \\
\text { re Schussabgabe zu ermöglichen } \\
\text { - Wildtierpassagen und Korridore zu beruhigen } \\
\end{array}$ \\
\hline 6 & gering & hoch & $\begin{array}{l}\text { Eignungsbereich für } \\
\text { kleinräumige Kom- } \\
\text { pensations- bzw. } \\
\text { Alternativangebote } \\
\text { zur Besucherlen- } \\
\text { kung } \\
\end{array}$ & $\begin{array}{l}\text { - Potenzieller Eignungsbereich für punktuelle } \\
\text { Atttraktivierungsangebote für Freizeitnutzer zur Besu- } \\
\text { cherlenkung nach vorheriger Prüfung } \\
\text { - Sicherung störungsarmer Rotwild-Kerngebiete in Berei- } \\
\text { chen mit hoher Vorkommensdichte andernorts }\end{array}$ \\
\hline 7 & mittel & gering & $\begin{array}{l}\text { Erhaltung geringer } \\
\text { Störungsintensität }\end{array}$ & $\begin{array}{l}\text { - Geringhalten der Erholungsnachfrage durch Besucher- } \\
\text { lenkungsmaßnahmen, mit Präferenz für „sanfte“ Maß- } \\
\text { nahmen und Anreizen für Besucher in wenig sensiblen } \\
\text { Gebieten } \\
\text { - Einrichtung von Ruhezonen für das Rotwild im Bereich } \\
\text { von Kernzonen }\end{array}$ \\
\hline 8 & gering & mittel & $\begin{array}{l}\text { „Business-as- } \\
\text { usual“-Bereich }\end{array}$ & $\begin{array}{l}\text { - Kein Rotwild-spezifischer Managementbedarf } \\
\text { - Vorbehaltsberich für relevante Ziele, die sich gegebe- } \\
\text { nenfalls aus Managementbedarf anderer Indikatorarten } \\
\text { ableiten lassen können } \\
\end{array}$ \\
\hline 9 & gering & gering & $\begin{array}{l}\text { Erhaltung Status } \\
\text { quo - kein Rotwild- } \\
\text { spezifischer Mana- } \\
\text { gementbedarf } \\
\end{array}$ & $\begin{array}{l}\text { - Erhaltung störungsarmen Naturraums im Sinne generel- } \\
\text { ler Nachhaltigkeitsziele des Biosphärenparks }\end{array}$ \\
\hline 0 & rotw & dfrei & $\begin{array}{l}\text { Vorrangbereich für } \\
\text { kleinräumige Kom- } \\
\text { pensations- bzw. } \\
\text { Alternativangebote } \\
\text { zur Besucherlen- } \\
\text { kung }\end{array}$ & $\begin{array}{l}\text { - Schwerpunktbereich für punktuelle } \\
\text { Atttraktivierungsangebote für Freizeitnutzer zur Besu- } \\
\text { cherlenkung } \\
\text { - Sicherung störungsarmer Rotwild-Kerngebiete in Berei- } \\
\text { chen mit hoher Vorkommensdichte andernorts }\end{array}$ \\
\hline
\end{tabular}

* Anmerkung: Ausprägungstyp der Konfliktpotenzialstufe 4 tritt in GIS-Modellierung nicht auf. 


\subsection{Rothirsch: Übersicht über Handlungsoptionen des Besuchermanagements und querschnittsorientierte Maßnahmenoptionen nach Konfliktpotenzialstu- fen}

In der nachstehenden Tabelle 44 werden die in Kap. 4.2 beschriebenen Maßnahmenoptionen aus den Bereichen Besuchermanagement und querschnittsorientierte Maßnahmen den Konfliktpotenzialstufen in Bezug auf das Rotwild zugeordnet. Die Nummerierung der Maßnahmen entspricht der Gliederung in Kap. 4.2. Die Zordnung gibt Hinweise darauf, welche Maßnahmen grundsätzlich besonders geeignet erscheinen, um in unterschiedlichen Konfliktpotenzialsituationen zum Konfliktmanagement beizutragen. Die unterschiedlichen Konfliktpotenzialstufen entsprechen den farblich differenzierten Konfliktpotenzialräumen in Abbildung 118. Die Tabelle ist als Entscheidungshilfe für die Maßnahmenauswahl und -priorisierung zu verstehen und sollte im Sinne eines Maßnahmenbaukastens benutzt werden. 
Tabelle 44: Besonders geeignete Maßnahmenoptionen (Besuchermanagement, Querschnittsmaßnahmen) je Konfliktpotenzialstufe für den Rothirsch.

\begin{tabular}{|c|c|c|c|c|c|c|c|c|c|c|c|}
\hline \multicolumn{12}{|c|}{$\begin{array}{l}\text { ROTHIRSCH } \\
\text { Zuordnung von Maßnahmenpräferenzen (Besuchermanagement, Querschnittsmaßnahmen) zu den Konfliktpotenzialausprägungen }\end{array}$} \\
\hline \multicolumn{2}{|c|}{ Konfliktpotenzialstufe } & 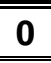 & 1 & 2 & 3 & $4^{*}$ & 5 & 6 & 7 & 8 & 9 \\
\hline \multicolumn{2}{|r|}{ Vorkommenswahrscheinlichkeit (Sensibilität) } & & hoch & hoch & mittel & hoch & mittel & gering & mittel & gering & gering \\
\hline \multicolumn{2}{|r|}{ Nutzungswahrscheinlichkeit (Erholungsnachfrage) } & & hoch & mittel & hoch & gering & mittel & hoch & gering & mittel & gering \\
\hline \multicolumn{12}{|c|}{ Maßnahmenoptionen } \\
\hline \multicolumn{12}{|c|}{\begin{tabular}{l|l} 
Nr. $^{*}$ & Bezeichnung \\
\end{tabular}} \\
\hline \multicolumn{12}{|c|}{ Besuchermanagement - Freizeit- und Erholungsaktivitäten } \\
\hline \multirow{2}{*}{\multicolumn{12}{|c|}{ Kleinräumige Maßnahmenoptionen }} \\
\hline $\boldsymbol{A}$ & Räumlich-zeitliche Nutzungsrestriktionen & & & & & & & & & & \\
\hline A1 & $\begin{array}{l}\text { Vereinheitlichung rechtlich verankerter Nutzungsverbote und -gebote (Kern- } \\
\text { zonen) }\end{array}$ & $\mathrm{x}$ & $\mathrm{x}$ & $\mathrm{x}$ & $\mathrm{x}$ & $\mathrm{x}$ & $\mathrm{x}$ & $\mathbf{x}$ & $\mathbf{x}$ & $\mathrm{x}$ & $\mathrm{x}$ \\
\hline A2 & Verzicht auf Ausbau der Wegeinfrastruktur & & $\mathrm{x}$ & $\mathbf{x}$ & $\mathbf{x}$ & $\mathbf{x}$ & (X) & & $\mathbf{x}$ & & $\mathbf{x}$ \\
\hline A3 & Verlegung bzw. Auflassung von Wegen und kernzonengerechtes Wegenetz & & $\mathbf{x}$ & $\mathbf{X}$ & $(\mathrm{X})$ & $\mathbf{X}$ & (X) & & & & \\
\hline A4 & $\begin{array}{l}\text { Freiwillige Vereinbarungen zu örtlich begrenzten, räumlichen und/oder zeitli- } \\
\text { chen Nutzungseinschränkungen }\end{array}$ & & $\mathrm{x}$ & $\mathrm{x}$ & $(\mathbf{X})$ & $\mathrm{x}$ & $(\mathrm{X})$ & & & & \\
\hline A5 & $\begin{array}{l}\text { Jagdrechtlich begründete Nutzungsrestriktionen (Wildschutzgebiete, befriste- } \\
\text { te jagdliche Sperrgebiete) }\end{array}$ & & $\mathrm{x}$ & $\mathrm{x}$ & $(\mathrm{X})$ & $\mathrm{x}$ & $(\mathrm{X})$ & & & & \\
\hline$B$ & Besucherlenkung durch De-Attraktivierung & & & & & & & & & & \\
\hline B1 & Landschaftsgestalterische Maßnahmen zur Verringerung der Zugänglichkeit & & $\mathbf{x}$ & $\mathbf{x}$ & $\mathbf{x}$ & $\mathbf{x}$ & $\mathbf{x}$ & & $\mathbf{x}$ & & \\
\hline B2 & Verzicht auf Schneeräumung & & $\mathbf{x}$ & $\mathbf{x}$ & $\mathbf{x}$ & $\mathbf{x}$ & $\mathbf{X}$ & & $\mathbf{x}$ & & \\
\hline B3 & Management von Infrastruktur mit Schleusenfunktion & & $\mathbf{x}$ & $\mathbf{x}$ & (X) & $\mathbf{x}$ & $(\mathrm{X})$ & & & & \\
\hline c & $\begin{array}{l}\text { Kleinräumige Kompensations- bzw. Alternativangebote zur Besucher- } \\
\text { lenkung }\end{array}$ & & & & & & & & & & \\
\hline $\mathrm{C} 1$ & Hundeauslaufbereiche & $\mathrm{x}$ & & & & & & $\mathbf{x}$ & & & \\
\hline $\mathrm{C} 2$ & Night Trails für Jogger & $\mathbf{x}$ & & & & & & $\mathbf{x}$ & & & \\
\hline $\mathrm{C} 3$ & Night Trails und Winter Trails für Mountainbiker & $\mathrm{X}$ & & & & & & $\mathrm{x}$ & & & \\
\hline $\mathrm{C} 4$ & Mountainbike Technik Trails & $\mathbf{x}$ & & & & & & $\mathbf{x}$ & & & \\
\hline $\mathrm{C5}$ & Mountainbike Downhill-Strecken & $\mathrm{X}$ & & & & & & $\mathrm{X}$ & & & \\
\hline C6 & Betreute Picknickplätze & $\mathbf{x}$ & & & & & & $\mathbf{x}$ & & & \\
\hline $\mathrm{C} 7$ & Wildlife Viewing-Einrichtungen (Schwarzstorch-Beobachungswarten) & & & & & & & & & & \\
\hline
\end{tabular}




\begin{tabular}{|c|c|c|c|c|c|c|c|c|c|c|c|}
\hline \multicolumn{12}{|c|}{$\begin{array}{l}\text { ROTHIRSCH } \\
\text { Zuordnung von Maßnahmenpräferenzen (Besuchermanagement, Querschnittsmaßnahmen) zu den Konfliktpotenzialausprägungen }\end{array}$} \\
\hline \multicolumn{2}{|c|}{ Konfliktpotenzialstufe } & 0 & 1 & 2 & 3 & $4 *$ & 5 & 6 & 7 & 8 & 9 \\
\hline & Vorkommenswahrscheinlichkeit (Sensibilität) & & hoch & hoch & mittel & hoch & mittel & gering & mittel & gering & gering \\
\hline & Nutzungswahrscheinlichkeit (Erholungsnachfrage) & & hoch & mittel & hoch & gering & mittel & hoch & gering & mittel & gering \\
\hline \multicolumn{12}{|c|}{ Maßnahmenoptionen } \\
\hline Nr.* ${ }^{*}$ & Bezeichnung & & & & & & & & & & \\
\hline$\overline{\mathrm{C} 8}$ & Verstärkte Instandhaltung und Pflege der bestehenden Wegeinfrastruktur & $\bar{x}$ & & & & & & $\bar{x}$ & & & \\
\hline$D$ & Verstärkte Information, Kontrolle und Vollzug in Schwerpunktbereichen & & & & & & & & & & \\
\hline D1 & Konsequente Durchsetzung des Wegegebotes in Kernzonen & & $\mathbf{x}$ & $\mathrm{x}$ & $\mathbf{x}$ & $\mathbf{x}$ & $\mathbf{x}$ & & $\mathbf{x}$ & & \\
\hline D2 & Verstärkte Information, Kontrolle und Sanktionierung in sensiblen Gebieten & & $\mathbf{x}$ & $\mathbf{x}$ & (X) & $\mathbf{x}$ & (X) & & $\mathbf{x}$ & & \\
\hline D3 & $\begin{array}{l}\text { Verstärkte Information, Kontrolle und Sanktionierung im Bereich von Kom- } \\
\text { pensations- und Alternativangeboten (siehe C) }\end{array}$ & & & & & & & $\mathrm{x}$ & & & \\
\hline$E$ & Teilraumbezogene Beurteilung von Zielpriorität und Managementbedarf & & & & $\mathbf{x}$ & & $\mathbf{x}$ & & & & \\
\hline E1 & $\begin{array}{l}\text { Prüfung des spezifischen Managementbedarfs und situationsabhängige } \\
\text { Priorisierung von Handlungsstrategien und Maßnahmenoptionen (A, B, D) in } \\
\text { Bezug auf das Rotwild oder andere Indikatorarten }\end{array}$ & & & & $\mathrm{x}$ & & $x$ & & & $(\mathrm{X})$ & \\
\hline \multicolumn{12}{|c|}{ Querschnittsorientierte Handlungsoptionen und Begleitmaßnahmen } \\
\hline $\boldsymbol{F}$ & Information, Kommunikation, Öffentlichkeitsarbeit & & & & & & & & & & \\
\hline F1 & Zielgruppenspezifische Kommunikation und Öffentlichkeitsarbeit & \multirow{5}{*}{\multicolumn{10}{|c|}{$\begin{array}{c}\text { Geeignete und kohärente Integration von relevanten Inhalten, um die Erreichung der jeweiligen Ma- } \\
\text { nagementziele je nach spezifischer Konfliktpotenzialausprägung und kleinräumiger Maßnahmen- } \\
\text { auswahl zu unterstützen }\end{array}$}} \\
\hline F1.1 & Interne Kommunikation & & & & & & & & & & \\
\hline F1.2 & Aus- und Weiterbildung & & & & & & & & & & \\
\hline F1.3 & Kommerzielle Angebote & & & & & & & & & & \\
\hline F1.4 & Kommunikationsschleusen & & & & & & & & & & \\
\hline & $\begin{array}{l}\text { - Nutzung von kleinräumigen Attraktivierungsangeboten }(\mathrm{C} 1-\mathrm{C} 6) \text { als Kom- } \\
\text { munikationsschleusen }\end{array}$ & & & & & & & $\mathrm{x}$ & & & \\
\hline F2 & $\begin{array}{l}\text { Biosphärenparkweite und zielgruppenspezifische Konzepte für Kommunikati- } \\
\text { on und Öffentlichkeitsarbeit }\end{array}$ & \multicolumn{10}{|c|}{$\begin{array}{c}\text { Geeignete und kohärente Integration von relevanten Inhalten, um die Erreichung der jeweiligen Ma- } \\
\text { nagementziele je nach spezifischer Konfliktpotenzialausprägung und kleinräumiger Maßnahmen- } \\
\text { auswahl zu unterstützen }\end{array}$} \\
\hline F3 & Schaffung von Kommunikationsstrukturen mit institutionalisierten Vertretern & \multicolumn{10}{|c|}{ Biosphärenparkweite Anwendung wird in jedem Fall empfohlen } \\
\hline
\end{tabular}




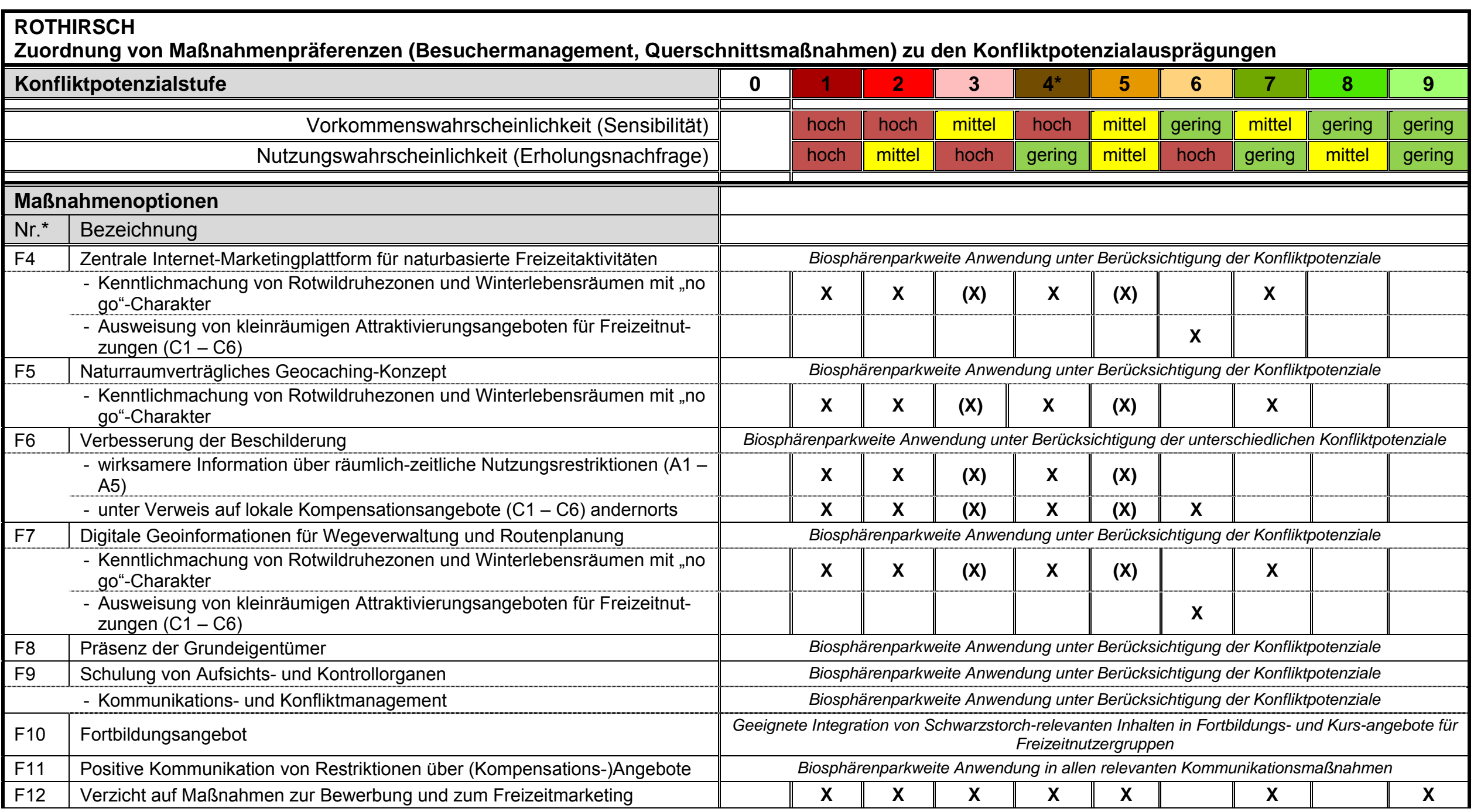




\begin{tabular}{|c|c|c|c|c|c|c|c|c|c|c|c|}
\hline \multicolumn{12}{|c|}{$\begin{array}{l}\text { ROTHIRSCH } \\
\text { Zuordnung von Maßnahmenpräferenzen (Besuchermanagement, Querschnittsmaßnahmen) zu den Konfliktpotenzialausprägungen }\end{array}$} \\
\hline \multicolumn{2}{|c|}{ Konfliktpotenzialstufe } & 0 & 1 & 2 & 3 & $44^{*}$ & 5 & 6 & 7 & 8 & 9 \\
\hline & Vorkommenswahrscheinlichkeit (Sensibilität) & & hoch & hoch & mittel & hoch & mittel & gering & mittel & gering & gering \\
\hline & Nutzungswahrscheinlichkeit (Erholungsnachfrage) & & hoch & mittel & hoch & gering & mittel & hoch & gering & mittel & gering \\
\hline \multicolumn{12}{|c|}{ Maßnahmenoptionen } \\
\hline $\mathrm{Nr}^{*}$ & Bezeichnung & & & & & & & & & & \\
\hline $\bar{G}$ & Kooperative Konfliktlösungsstrategien & & & & & & & & & & \\
\hline \multicolumn{2}{|c|}{ G1 - G4 (Details siehe Kap. 4.1.3.2.2) } & & $\mathbf{x}$ & $\mathbf{x}$ & $\mathbf{x}$ & $\mathbf{x}$ & $\mathbf{x}$ & $\mathbf{x}$ & $(\mathrm{X})$ & & \\
\hline \multicolumn{2}{|r|}{$\begin{array}{l}\text { - zur Aushandlung freiwilliger Vereinbarungen zu örtlich begrenzten, räumli- } \\
\text { chen und/oder zeitlichen Nutzungsrestriktionen für den Freizeitbetrieb (A1 } \\
\text { - A5) }\end{array}$} & & $x$ & $x$ & $(\mathrm{X})$ & $x$ & $(\mathrm{X})$ & & $(\mathrm{X})$ & & \\
\hline \multirow{2}{*}{\multicolumn{2}{|c|}{$\begin{array}{l}\text { - zur Standortfindung und Entwicklung kleinräumiger Kompensations- bzw. } \\
\text { Alternativangebote für Freizeitaktivitäten }(\mathrm{C} 1-\mathrm{C}) \\
\text { - zur Vereinbarung von Maßnahmen, die verstärkte sektorübergreifende Ko- } \\
\text { ordination zwischen Landnutzergruppen erfordern }\end{array}$}} & & & & & & & $x$ & & & \\
\hline & & & $\mathrm{x}$ & $\mathrm{x}$ & $\mathrm{x}$ & $\mathrm{x}$ & $\mathrm{x}$ & $x$ & $(\mathrm{X})$ & & \\
\hline$H$ & Kontrolle und Vollzug von Verhaltensregeln & \multirow{2}{*}{\multicolumn{10}{|c|}{$\begin{array}{l}\text { Biosphärenparkweite Anwendung wird empfohlen (zu Schwerpunktbereichen für Kontrolle und Voll- } \\
\text { zug siehe D) }\end{array}$}} \\
\hline \multicolumn{2}{|c|}{$\mathrm{H} 1$ - H2 (Details siehe Kap. 4.1.3.2.2) } & & & & & & & & & & \\
\hline$I$ & Biosphärenparkweite Verhaltensregeln & \multirow{2}{*}{\multicolumn{10}{|c|}{$\begin{array}{c}\begin{array}{c}\text { Biosphärenparkweite Anwendung wird unabhängig von der jeweiligen Konfliktpotenzialausprägung } \\
\text { empfohlen }\end{array} \\
\end{array}$}} \\
\hline 11 & Einheitliche Rahmen-Nutzungsregeln im Biosphärenpark & & & & & & & & & & \\
\hline J & $\begin{array}{l}\text { Maßnahmenauswahl in Abhängigkeit vom Ergebnis der Prüfung des } \\
\text { spezifischen Managementbedarfs (vgl. E1) }\end{array}$ & & & & $\mathrm{x}$ & & $x$ & & & $(X)$ & \\
\hline J1 & $\begin{array}{l}\text { Flexible und geeignete Ausgestaltung von querschnittsorientierten Maßnah- } \\
\text { men in Abhängigkeit vom Prüfergebnis zum Managementbedarf und der hie- } \\
\text { raus abgeleiteten kleinräumigen Maßnahmenauswahl (vgl. E1) }\end{array}$ & & & & $\mathrm{x}$ & & $\mathrm{x}$ & & & $(\mathrm{X})$ & \\
\hline \multicolumn{12}{|c|}{ Generelle Maßnahmen für weitere Landnutzergruppen } \\
\hline \multirow{2}{*}{$\begin{array}{ll}K \\
\mathrm{~K} 1\end{array}$} & Raumplanung, Infrastrukturplanung & & & & & & & & & & \\
\hline & Forcierte Umsetzung von Zielen einer nachhaltigen Siedlungsentwicklung & \multicolumn{10}{|c|}{ Biosphärenparkweite Anwendung unter Berücksichtigung der unterschiedlichen Konfliktpotenziale } \\
\hline K2 & $\begin{array}{l}\text { Verstärkte Sicherung von störungsarmen, ökologisch bedeutsamen Frei- und } \\
\text { Grünräumen sowie von ökologischen Korridoren und Migrationsachsen }\end{array}$ & $(\mathrm{X})$ & $x$ & $x$ & $x$ & $x$ & $x$ & & $x$ & & \\
\hline K3 & $\begin{array}{l}\text { Errichtung und raumplanerische Absicherung von ökologischen } \\
\text { Querungshilfen für Wildtiere }\end{array}$ & $(\mathrm{X})$ & $x$ & $\mathrm{x}$ & $x$ & $x$ & $x$ & & $x$ & & \\
\hline K4 & $\begin{array}{l}\text { Verstärkte Prüfung von Entwicklungsvorhaben im Rahmen der Anwendung } \\
\text { von Prüfinstrumenten (UVP, SUP, NVP) }\end{array}$ & & $\mathrm{x}$ & $\mathrm{x}$ & $x$ & $\mathrm{x}$ & $x$ & & $x$ & & $(\mathrm{X})$ \\
\hline
\end{tabular}




\begin{tabular}{|c|c|c|c|c|c|c|c|c|c|c|c|}
\hline \multirow{2}{*}{\multicolumn{2}{|c|}{$\begin{array}{l}\text { ROTHIRSCH } \\
\text { Zuordnung von Maßnahı } \\
\text { Konfliktpotenzialstufe } \\
\end{array}$}} & & na & ) & K & $\operatorname{tp}$ & ia & rä & & & \\
\hline & & 0 & 1 & 2 & 3 & $4^{*}$ & 5 & 6 & 7 & 8 & 9 \\
\hline \multirow{2}{*}{\multicolumn{2}{|c|}{$\begin{array}{l}\text { Vorkommenswahrscheinlichkeit (Sensibilität) } \\
\text { ungswahrscheinlichkeit (Erholungsnachfrage) }\end{array}$}} & & \multirow{2}{*}{$\frac{\text { hoch }}{\text { hoch }}$} & \multirow{2}{*}{$\begin{array}{l}\text { hoch } \\
\text { mittel }\end{array}$} & \multirow{2}{*}{$\begin{array}{c}\text { mittel } \\
\text { hoch } \\
\end{array}$} & \multirow{2}{*}{$\begin{array}{l}\text { hoch } \\
\text { gering }\end{array}$} & \multirow{2}{*}{$\begin{array}{l}\text { mittel } \\
\text { mittel }\end{array}$} & \multirow{2}{*}{$\frac{\text { gering }}{\text { hoch }}$} & \multirow{2}{*}{$\begin{array}{ll}\text { mittel } \\
\text { gering } \\
\end{array}$} & \multirow{2}{*}{$\begin{array}{c}\text { gering } \\
\text { mittel }\end{array}$} & \multirow{2}{*}{$\begin{array}{l}\text { gering } \\
\text { gering }\end{array}$} \\
\hline & & & & & & & & & & & \\
\hline \multicolumn{12}{|c|}{ Maßnahmenoptionen } \\
\hline $\mathrm{Nr}^{*}$ & \multicolumn{11}{|l|}{ Bezeichnung } \\
\hline K5 & $\begin{array}{l}\text { Schaffung eines Flächenpools für Ausgleichsmaßnahmen und Aktivierung } \\
\text { geeigneter Flächen zur Kompensation von Eingriffen in den Naturraum }\end{array}$ & $\mathbf{x}$ & & & & & & $\mathrm{x}$ & & & \\
\hline K6 & $\begin{array}{l}\text { Aktive und koordinierende Rolle der Raumordnung bei der Standortplanung } \\
\text { und Raumverträglichkeitsprüfung von neuen bzw. alternativen Angeboten für } \\
\text { Freizeitaktivitäten (C) }\end{array}$ & & & & & & & $\mathbf{x}$ & & & \\
\hline $\mathbf{L}$ & \multicolumn{11}{|l|}{ Sektorübergreifende Kooperation aller Landutzungsbereiche } \\
\hline L1 & $\begin{array}{l}\text { Verbesserte und abgestimmte Öffentlichkeitsarbeit, Bewusstseinsbildung, } \\
\text { Kommunikation und Information }\end{array}$ & \multicolumn{10}{|c|}{ Biosphärenparkweite Anwendung und Integration von Rotwild-relevanten Inhalten } \\
\hline L2 & $\begin{array}{l}\text { Verstärkte Mitarbeit aller Landnutzergruppen bei der Ausarbeitung des Ma- } \\
\text { nagementplans für den Biosphärenpark Wienerwald }\end{array}$ & \multicolumn{10}{|c|}{ Biosphärenparkweite Anwendung unter Berücksichtigung der unterschiedlichen Konfliktpotenziale } \\
\hline L3 & $\begin{array}{l}\text { Aktive Beteiligung und Einbeziehung von Landnutzerinteressen in } \\
\text { partizipative Prozesse zur Entwicklung von kleinräumigen } \\
\text { Attraktivierungsangeboten zur Besucherlenkung } \\
\end{array}$ & & & & & & & $\mathbf{x}$ & & & \\
\hline \multicolumn{12}{|c|}{ Monitoring und adaptives Management } \\
\hline$M$ & Besuchermonitoring & & & & & & & & & & \\
\hline M1 & $\begin{array}{l}\text { Besuchermonitoring mit Hilfe anonymisierter Bewegungsdaten von Handy- } \\
\text { nutzerlnnen }\end{array}$ & & $\mathrm{x}$ & $\mathbf{x}$ & $\mathrm{x}$ & $\mathrm{x}$ & & $(\mathrm{X})$ & & & \\
\hline M2 & Beobachtungen und Zählungen von Besucherströmen & & $\mathbf{X}$ & $\mathbf{X}$ & $\mathbf{x}$ & $\mathbf{X}$ & & $\mathbf{X}$ & & & \\
\hline M3 & Befragungen & & \multicolumn{9}{|c|}{$\begin{array}{c}\text { Durchführung wird biosphärenparkweit empfohlen; Befragungsdesign und Auswertung soll- } \\
\text { ten unterschiedliche Konfliktpotenziale und entsprechende Managementoptionen berück- } \\
\text { sichtigen }\end{array}$} \\
\hline$N$ & Erfolgskontrolle zu vereinbarten Nutzungsregeln & & & & & & & & & & \\
\hline N1 & Monitoring der Wirksamkeit von getroffenen Nutzungsvereinbarungen & & $\mathbf{X}$ & $\mathbf{X}$ & $\mathbf{X}$ & $\mathbf{X}$ & $\mathbf{X}$ & $\mathbf{X}$ & & & \\
\hline
\end{tabular}

Zeichenerklärung: $\quad X$... prioritäre Managementoption; $(X)$... mit Vorbehalt geeignete bzw. weniger prioritäre Managementoption 


\subsection{Spezifische Maßnahmenoptionen für weitere Landnutzergruppen in Bezug auf den Rothirsch}

\section{Raumplanung, Infrastrukturplanung}

Errichtung und raumplanerische Absicherung von technischen Wildquerungshilfen für das Rotwild an A1 und A21: Gegenwärtig fungieren die beiden Autobahntrassen der A1 und A21 als Barriere für wandernde Wildtiere bzw. ist im Fall der A21 die wildtierökologische Durchlässigkeit eines bestehenden Talübergangs nicht genau bekannt. Das Rotwild ist als hochmobile Tierart mit großräumigem Raumnutzungsverhalten von der Barrierewirkung dieser beiden hochrangigen Verkehrsträger besonders betroffen. Darüber hinaus wird der gesamte Bereich des Wienerwalds nördlich der A1 derzeit aufgrund forstwirtschaftlicher Zielsetzungen der Grundeigentümer faktisch rotwildfrei gehalten. In Summe ergibt sich aus diesen Rahmenbedingungen, dass die Funktionsfähigkeit des Wienerwalds als großräumiger Trittstein- bzw. Korridorlebensraum zwischen Alpen und Karpaten derzeit nicht oder nur sehr eingeschränkt gegeben ist. Um die - auch überregionale - Lebensraumvernetzung und den Populationsaustausch für das Rotwild wiederherzustellen und zu verbessern, wäre die Errichtung von für das Rotwild geeigneten technischen Wildquerungshilfen (Wildtierpassagen, Grünbrücken, Wildbrücken bzW. -durchlässe) an der A1 und A21 erforderlich. Soweit es die A1 betrifft, wäre für die großräumige Populationsvernetzung zudem ein Abrücken von der derzeitigen Grundeigentümerpolitik der Nicht-Duldung des Rotwildes im gesamten Bereich nördlich der A1 erforderlich. Die Standortwahl, Dimensionierung und baulich-vegetationstechnische Gestaltung von rotwildtauglichen Wildtierpassagen sollte sich an den Norm- bzw. Orientierungswerten für solche Bauwerke gemäß RVS 04.03.12 (BMVIT \& ÖFSV, 2007) bzw. an Völk et al. (2001) orientieren, um die Annahme durch das Rotwild zu gewährleisten. Es ist davon auszugehen, dass für die Indikatorwildart Rotwild geeignete Querungseinrichtungen gleichzeitig für die meisten anderen heimischen Säugetierarten geeignet sind. Eine wesentliche raumplanerische Begleitmaßnahme liegt in der Sicherung der dauerhaften Funktionsfähigkeit solcher Querungshilfen für Wildtiere durch entsprechende überörtliche Ausweisung geeigneter Schutzzonen sowie in der konsequenten Durchsetzung von Flächenwidmungs- und Nutzungsverboten im Umfeld der betreffenden Passagen und der durch diese verbundenen Wildtierkorridore. Zur Verbesserung der Annahmewahrscheinlichkeit sind weiters Maßnahmen zur Landschaftsgestaltung und zur Besucherlenkung sowie ein Bejagungsverzicht im Bereich der betreffenden Engstellen erforderlich.

\section{Jagdwirtschaft}

Effiziente und störungsarme Bejagung: In Gebieten mit vergleichsweise dichtem Rotwildvorkommen und guter Lebensraumeignung für das Rotwild sollte auch die Jagdausübung durch eine entsprechende Ausrichtung der Bejagungsstrategie dazu beeitragen, nicht notwendige Störungen des Lebensryhthmus und des Raumnutzungsverhaltens des Rotwildes zu verringern bzw. gering zu halten. Da sich gerade in Bezug auf das erhöht störungssensible Rotwild Störungen durch Jagddruck und durch Freizeitaktivitäten im Wildlebensraum wechselseitig verstärken können, sollte insbesondere in Gebieten mit hoher Rotwilddichte und gleichzeitig intensiver Freizeitnutzung der Jagddruck ganzjährig weitest möglich minimiert werden. Um eine effiziente und störungsarme Bejagung des Rotwilds mit geringstmöglichem Jagddruck zu ermöglichen, ist die verstärkte Anwendung von Bejagungsstrategien wie der Intervalljagd mit ausgeprägten Jagdruhezeiten zu empfehlen. Beitragen können hierzu weiters eine möglichst kurze Bejagungszeit, die in Ext- 
remfällen auch auf nur wenige Jagdtage pro Jahr reduziert werden kann, sowie die zeitliche Limitierung bzw. der völlige Verzicht auf die Nachtjagd auf Schwarzwild in Rotwildkerngebieten. In siedlungsfernen Rotwildkerngebieten kann die Schwarzwildregulation anstelle der Bejagung in der Nacht durch Bewegungsjagden auch tagsüber kompensiert werden. Ruhegebiete, d. h. störungsberuhigte Einstände und Rückzugsräume für das Rotwild, sollten auch von jagdlicher Seite nicht beunruhigt werden.

Einrichtung von Ruhegebieten für das Rotwild: Um einerseits die Besiedlungsattraktivität von bestehenden Rotwildkerngebieten sowie von Winterlebensräumen zu erhalten und andererseits eine effiziente Bejagbarkeit des Rotwilds bei geringem Jagddruck zu gewährleisten, ist die Schaffung bzw. Erhaltung von ungestörten Ruhe- und Rückzugsräumen für das Rotwild notwendig. Von Seiten des Freizeit- und Erholungsmanagements kann dies durch Kanalisierung von Freizeitaktivitäten, durch alle Maßnahmen, die zu einer Extensivierung und De-Attraktivierung für Erholungstätigkeiten beitragen, sowie durch freiwillige Nutzungsvereinbarungen mit Jagdwirtschaft und Grundeigentümern erfolgen (vgl. Kap. 4.2.1.1, Maßnahmengruppen A und B). Gleichzeitig sollte in Ruhezonen auch keinerlei Bejagung durchgeführt werden. Kernzonen des Biosphärenparks sind bei entsprechender Habitateignung zur Einrichtung von Ruhezonen besonders geeignet. In jedem Fall sind Rotwildruhezonen in Jagdkonzepte und revierübergreifende Bejagungsrichtlinien zu integrieren. Die Einrichtung von Ruhezonen sollte in allen Rotwildkerngebieten mit hoher Vorkommenswahrscheinlichkeit erfolgen. In Gebieten mit gleichzeitig intensiver Freizeitnutzung wird es hierzu besonderer, mit der Jagdwirtschaft abgestimmter Maßnahmen zur Besucherlenkung bedürfen, wohingegen in Gebieten mit geringer Freizeitnutzung die Umsetzung leichter durchführbar erscheint. Hauptwechsel des Rotwilds und insbesondere das Umfeld von technischen Wildquerungseinrichtungen über Infrastrukturbarrieren sind in jedem Fall jagdfrei zu halten.

Vereinbarung definierter Jagdtage mit anderen Landnutzergruppen: Vor allem in Rotwildkerngebieten mit hoher Freizeitnutzungsintensität sowie in Siedlungsnahlage kann es vorteilhaft sein, die Festlegung definierter Jagdtage bzw. Jagdzeiten mit Vertretern von Freizeit- und Erholungsnutzergruppen, Gemeinden und Grundeigentümern anzustreben. Derartige Vereinbarungen müssen im Rahmen von kooperativen Konfliktlösungsmodellen (vgl. Kap. 4.2.1.2, Maßnahmengruppe G) ausgehandelt werden und wären als freiwilliges „gentleman's agreement“ zu betrachten. Während solcher definierten Jagdtage sollten in Freizeitaktivitäten in den betreffenden Bereichen weitest möglich unterbleiben. Diese Maßnahme würde eine effiziente jagdliche Abschusserfüllung bei geringem Jagddruck während des restlichen Jahres ermöglichen und die Sicherheit der Schussabgabe unterstützen.

Ausrichtung der Bejagungsstrategie an der Wildschadenanfälligkeit von Waldbeständen: Aktuell sind im Biosphärenpark keine gravierenden Schälschadenprobleme durch Rotwild festzustellen. Grundsätzlich ist der Bedarf nach Berücksichtigung des Wildschadenrisikos bei der Bejagung beim Rotwild daher gegenwärtig deutlich geringer als beim Schwarzwild. Da sich aber sowohl Rotwildpopulationen als auch forstliche Habitate im Lauf der Zeit verändern, sind im selben Maße zukünftig auch Veränderungen des Wildschadenrisikos und der Schälschadensituation nicht auszuschließen. In Gebieten mit erhöhter Wildschadenanfälligkeit ist die Bejagungsstrategie gezielt auf die forstliche Schadenvermeidung auszurichten. Schwerpunktbejagungen und deren regulierende sowie vergrämende Wirkung auf das Rotwild sollten jedoch auf die unmittelbaren Wildschadenrisikogebiete konzentriert bleiben, $d$. h. alle anderen Teilflächen des Rotwildkerngebietes sollen ihre Habitatattraktivität behalten. 
Revierübergreifende Abstimmung der Bejagung: Revierübergreifende Bejagungsrichtlinien sind gerade für das Rotwild als großräumig agierende Wildart besonders erforderlich. Dieser Bedarf wird weiters durch die im österreichweiten Vergleich geringen durchschnittlichen Reviergrößen in weiten Teilen des Wienerwalds verstärkt. Die Bejagung muss sich an der Lebensraumnutzung von Wildarten und nicht an den Reviergrenzen orientieren. Eine revierübergreifende Abstimmung ist für die meisten der oben angeführten jagdlichen Maßnahmenoptionen vorteilhaft; insbesondere für die Einrichtung von Ruhezonen für das Rotwild und die Minimierung des Jagddrucks in größeren Rotwildkerngebieten erscheint sie unabdingbar.

\section{Forstwirtschaft}

Berücksichtigung der Wildschadenanfälligkeit (Schälschadenrisiko) bei der Waldbewirtschaftung: Die forstliche Bewirtschaftungsweise (Betriebsform, Verjüngungsverfahren, Baumartenwahl, Pflegeeingriffe, Forstwegebau, etc.) hat direkten und indirekten Einfluss auf die Wildschadenanfälligkeit des Waldes. Hinsichtlich des direkten Einflusses ist zu beachten, dass manche Baumartenmischungen sowie bestimmte Sukzessionsstadien schälschadenanfälliger sind als andere. Indirekt steuert die Waldbewirtschaftung in quantitativer und qualitativer Hinsicht maßgeblich das für das Wild verfügbare Nahrungsangebot sowie die nahrungsunabhängigen Habitatfaktoren Wohnraum und Deckung (Schutz gegen Klimaeinflüsse und Störeinflüsse) im Waldlebensraum. Die Wildschadenanfälligkeit des Waldes ist in der Regel umso höher, je geringer das Nahrungsangebot im Verhältnis zum nahrungsunabhängigen Besiedlungsanreiz (Wohnraum, Deckung) für die Tiere ist. Insbesondere durch unterschiedliche forstliche Maßnahmen, die zur Äsungsverbesserung für das Rotwild beitragen, kann die Forstwirtschaft daher zur Verringerung der Wildschadenanfälligkeit in Rotwildkerngebieten und Winterlebensräumen beitragen. Durch rechtzeitige auflichtungsfördernde Pflegeeingriffe, wie Jungwuchs- und Dickungspflege, kann die Äsungssituation verbessert und gleichzeitig Klimaschutz und Feindschutz reduziert werden, was zu einem günstigeren Verhältnis von Besiedlungsanreiz zu Nahrungsangebot führt und die Wildschadenanfälligkeit des Waldes reduziert. Vor allem in den Wintereinständen des Rotwildes und im Bereich allfälliger Fütterungsstandorte sollte im Rahmen eines angepassten Waldbaus auf schälschadenresistente Waldbestände geachtet werden. Forstliche Maßnahmen zur Verringerung des Schälschadenrisikos sollten eng mit der jagdlichen Maßnahmenplanung abgestimmt werden.

Erhaltung rotwildgeeigneter Rückzugshabitate und rechtzeitige Entwicklung von Ausweichhabitaten: Vergleichsweise hohes Rotwildvorkommen bei gleichzeitig hoher Freizeitnutzungsintensität weist darauf hin, dass funktionierende Rückzugshabitate vorhanden sind. Solche Rückzugsgebiete sollten, soweit es forstwirtschaftlich gerechtfertigt ist, durch eine entsprechende Waldbewirtschaftung erhalten werden. $\mathrm{Da}$ die Habitateignung sich im Zuge der Waldentwicklung und forstlichen Bewirtschaftung im Zeitverlauf verändert, müssen jedoch auch rechtzeitig geeignete und schadenstolerante Ausweichhabitate etabliert werden.

Überprüfung forstwirtschaftlicher Zielsetzungen in Bezug auf derzeit rotwildfreie Gebiete bzw. Teilräume mit geringer Vorkommensdichte: Für den Fall, dass das Rotwild in denjenigen Gebieten südlich der Autobahntrasse der A1, in denen diese Wildart derzeit nur in geringer Dichte auftritt, stärker als jagdlich nutzbare Art etabliert werden soll, wäre vielfach eine gezielte waldbauliche Habitatgestaltung im Sinne der Lebensraumansprüche des Rotwilds sowie unter Berücksichtigung der nötigen Schadenstoleranz erforderlich. Bei gleichzeitigem Vorliegen intensiver Freizeitaktivitäten würde zudem eine star- 
ke Extensivierung bzw. Kanalisierung des Freizeitbetriebs nötig. Für den gesamten, gegenwärtig aufgrund forstwirtschaftlicher Zielsetzungen weitgehend „rotwildfrei“ gehaltenen Teil des Biosphärenparks nördlich der A1 wäre zu überprüfen, ob diese Politik der Grundeigentümer beibehalten oder modifiziert werden soll. Die Nicht-Duldung des Rotwilds in diesem nördlichen Teil des Wienerwalds fungiert derzeit ebenso wie die Trasse der A1 faktisch als Barriere für die populationsgenetische Vernetzung mit dem gesamten Bereich nördlich der Donau (als Teil des Alpen-Karpaten-Korridors). Falls die Ansiedlung des Rotwilds im derzeit faktisch rotwildfreien Bereich zukünftig erwünscht sein sollte, wäre hier ebenfalls auf eine rotwildgeeignete und schadenstolerante forstliche Habitatgestaltung zu achten.

\section{Landwirtschaft}

Erhaltung von Äsungsflächen für das Rotwild: Insbesondere in Teilräumen mit hoher Vorkommensdichte des Rotwilds, d.h. in Kerngebieten und in der Umgebung von Ruhezonen, kann die Landwirtschaft durch den Erhalt von Grünlandflächen und den Verzicht auf Landnutzungsänderungen zur Bereitstellung von Äsungsangebot, und damit zur Reduktion der Wildschadenanfälligkeit von Waldkulturen, beitragen.

\section{Alle Nutzergruppen}

Betreffend die aktive Beteiligung aller Landnutzergruppen an partizipativen Prozessen zur Entwicklung von kleinräumigen Kompensations- bzw. Alternativangeboten für Freizeitnutzergruppen (vgl. Kap. 4.2.2, Empfehlung L3) siehe die Übersichtsmatrix in Tabelle 44 (Kap. 4.3.2.2.5). 


\subsection{Rothirsch: Übersicht über spezifische Maßnahmenoptionen für weitere Landnutzergruppen nach Konfliktpotenzialstufen}

In der nachstehenden Tabelle 45 werden die im vorangehenden Kap. 4.3.2.2.6 beschriebenen Maßnahmenoptionen für einzelne Landnutzergruppen sowie die in Kap. 4.2.3 beschriebenen Maßnahmen zum Monitoring von Wildtierpopulationen und -lebensräumen den Konfliktpotenzialstufen in Bezug auf den Rothirsch zugeordnet. Die Zordnung gibt Hinweise darauf, welche Maßnahmen grundsätzlich besonders geeignet erscheinen, um in unterschiedlichen Konfliktpotenzialsituationen zum Konfliktmanagement beizutragen. Die unterschiedlichen Konfliktpotenzialstufen entsprechen den farblich differenzierten Konfliktpotenzialräumen in Abbildung 118. Die Tabelle ist als Entscheidungshilfe für die Maßnahmenauswahl und -priorisierung zu verstehen und sollte im Sinne eines Maßnahmenbaukastens benutzt werden. 
Tabelle 45: Besonders geeignete sektorale Maßnahmenoptionen je Konfliktpotenzialstufe für das Rotwild.

\section{ROTHIRSCH}

Zuordnung von spezifischen Maßnahmenoptionen für Landnutzergruppen zu den Konfliktpotenzialausprägungen

\begin{tabular}{|c|c|c|c|c|c|c|c|c|c|c|}
\hline Konfliktpotenzialstufe & $\mathbf{0}$ & 1 & 2 & 3 & $4^{*}$ & 5 & 6 & 7 & 8 & 9 \\
\hline Vorkommenswahrscheinlichkeit (Sensibilität) & & hoch & hoch & mittel & hoch & mittel & gering & mittel & gering & gering \\
\hline Nutzungswahrscheinlichkeit (Erholungsnachfrage) & & hoch & mittel & hoch & gering & mittel & hoch & gering & mittel & gering \\
\hline \begin{tabular}{l|l} 
Nr. $^{*}$ & Bezeichnung \\
\end{tabular} & & & & & & & & & & \\
\hline Weitere Landnutzergruppen & & & & & & & & & & \\
\hline Raumplanung, Infrastrukturplanung & & & & & & & & & & \\
\hline Jagdwirtschaft & & & & & & & & & & \\
\hline Effiziente und störungsarme Bejagung & & $\mathbf{x}$ & $\mathbf{x}$ & $\mathrm{x}$ & $\mathrm{x}$ & $\mathbf{x}$ & & $(\mathrm{X})$ & & \\
\hline Einrichtung von Ruhegebieten für das Rotwild & & $\mathbf{x}$ & $\mathbf{x}$ & $\mathbf{x}$ & $\mathrm{x}$ & $\mathbf{x}$ & & & & \\
\hline Vereinbarung definierter Jagdtage bzw. -tageszeiten mit anderen Landnutzergruppen & & $\mathbf{x}$ & $\mathbf{x}$ & $\mathbf{x}$ & & $(\mathrm{X})$ & & & & \\
\hline $\begin{array}{l}\text { Ausrichtung der Bejagungsstrategie an der Wildschadenanfälligkeit von Waldbestän- } \\
\text { den }\end{array}$ & & $(\mathrm{X})$ & $(\mathrm{X})$ & $(\mathrm{X})$ & (X) & $(\mathrm{X})$ & & (X) & & \\
\hline Revierübergreifende Abstimmung der Bejagung & & $\bar{x}$ & $\bar{x}$ & $\bar{x}$ & $\bar{x}$ & $\mathrm{X}$ & & $\mathbf{X}$ & & \\
\hline $\begin{array}{l}\text { Überprüfung forstwirtschaftlicher Zielsetzungen in Bezug auf derzeit rotwildfreie Ge- } \\
\text { biete bzw. Teilräume mit geringer Vorkommensdichte }\end{array}$ & $\mathrm{x}$ & & & & & & & & & \\
\hline Landwirtschaft & & & & & & & & & & \\
\hline Erhaltung von Äsungsflächen für das Rotwild & & $\mathbf{x}$ & $\mathbf{x}$ & $\mathbf{x}$ & $\mathbf{x}$ & $\mathbf{x}$ & & $\mathbf{x}$ & & \\
\hline Monitoring und adaptives Management & & & & & & & & & & \\
\hline Erfolgskontrolle zu vereinbarten Nutzungsregeln & & & & & & & & & & \\
\hline Monitoring der Wirksamkeit von getroffenen Nutzungsvereinbarungen & & $\mathbf{x}$ & $\mathbf{X}$ & $\mathbf{X}$ & $\mathbf{X}$ & $\mathbf{X}$ & $\mathbf{X}$ & & & \\
\hline
\end{tabular}




\begin{tabular}{|c|c|c|c|c|c|c|c|c|c|c|c|}
\hline \multirow{2}{*}{\multicolumn{2}{|c|}{$\begin{array}{l}\text { ROTHIRSCH } \\
\text { Zuordnung von spezifischen Maßnahmenoptionen für Landnutzergruppe } \\
\text { Konfliktpotenzialstufe }\end{array}$}} & & & & & & & & & & \\
\hline & & \multirow[t]{3}{*}{0} & 1 & 2 & 3 & $4^{*}$ & 5 & 6 & 7 & 8 & 9 \\
\hline & Vorkommenswahrscheinlichkeit (Sensibilität) & & hoch & hoch & mittel & hoch & mittel & gering & mittel & gering & gering \\
\hline & Nutzungswahrscheinlichkeit (Erholungsnachfrage) & & hoch & mittel & hoch & gering & mittel & hoch & gering & mittel & gering \\
\hline \multicolumn{12}{|c|}{ Maßnahmenoptionen } \\
\hline \multicolumn{12}{|c|}{\begin{tabular}{l|l} 
Nr.*$^{*}$ & Bezeichnung \\
\end{tabular}} \\
\hline 0 & Monitoring von Wildtierpopulationen und -lebensräumen & & & & & & & & & & \\
\hline O1 & Einrichtung eines regelmäßigen Schwarzstorch-Monitorings & & & & & & & & & & \\
\hline $\mathrm{O} 2$ & Fortführung des Ziesel-Monitorings & & & & & & & & & & \\
\hline $\mathrm{O} 3$ & Monitoring von Schalenwildeinfluss auf die Waldvegetation & & $\mathbf{x}$ & $\mathrm{x}$ & $\mathbf{x}$ & $\mathbf{x}$ & $\mathbf{x}$ & & $\mathbf{x}$ & & \\
\hline $\mathrm{O} 4$ & Monitoring von Wildtierbeständen, Wildabschüssen und Fallwild & & $\mathbf{X}$ & $\mathrm{X}$ & $\mathbf{x}$ & $\mathbf{x}$ & $\mathbf{X}$ & $\mathrm{X}$ & $\mathbf{X}$ & $\mathbf{x}$ & $\mathbf{x}$ \\
\hline O5 & Zusätzliches Monitoring spezifischer Wildtierindikatorarten & & & & & & & & & & \\
\hline $\mathrm{O} 6$ & $\begin{array}{l}\text { Integration eines Wildtier-Monitorings in das allgemeine Biodiversitäts- } \\
\text { Monitoring }\end{array}$ & & $\mathbf{X}$ & $\mathbf{x}$ & $\mathbf{X}$ & $\mathbf{X}$ & $\mathbf{X}$ & $\mathbf{X}$ & $\mathbf{X}$ & $\mathbf{X}$ & $\mathbf{X}$ \\
\hline $\mathrm{O} 7$ & Monitoringpflicht in Natura 2000-Gebieten & & $x$ & $x$ & $\mathbf{x}$ & $x$ & $\mathbf{x}$ & $x$ & $x$ & $x$ & $x$ \\
\hline $\mathrm{O} 8$ & Monitoring der Wiesen & & $\mathbf{x}$ & $\mathbf{x}$ & $\mathbf{x}$ & $\mathbf{x}$ & $\mathbf{x}$ & $\mathbf{x}$ & $\mathbf{x}$ & $\mathrm{X}$ & $\mathbf{x}$ \\
\hline
\end{tabular}

Zeichenerklärung: $\quad$ X ... prioritäre Managementoption; $(\mathbf{X})$... mit Vorbehalt geeignete bzw. weniger prioritäre Managementoption 


\subsubsection{Wildschwein}

Die Hinweise und Entscheidungshilfen dieses Kapitels 4.3.2.3 zu den Zielen und zur Maßnahmenauswahl für das Konfliktmanagement beziehen sich ausschließlich auf die Indikatorart Wildschwein. Es ist darauf hinzuweisen, dass jedes Konfliktmanagement in konkreten Teilräumen des Biosphärenparks stets auch Konfliktpotenziale und Managementbedarf in Bezug auf die anderen Indikatorarten berücksichtigen und auf deren Zusammenschau basieren sollte.

\subsection{Modellierung des Konfliktpotenzials im GIS}

Die nachfolgende Kartendarstellung (Abbildung 120) zeigt neun unterschiedliche Ausprägungsformen des Konfliktpotenzials zwischen der Indikatorart Wildschwein und der Summe aller Indikator-Freizeitaktivitäten im Biosphärenpark Wienerwald. Das Konfliktpotenzial ergibt sich aus der Kombination der summierten Nutzungswahrscheinlichkeit aller Freizeitaktivitäten mit der Vorkommenswahrscheinlichkeit des Schwarzwilds.

Die Bezeichnung „-wahrscheinlichkeit“ im Zusammenhang mit Indikatorart und Indikatoraktivitäten wird verwendet, um auszudrücken, dass es sich um GIS-Modellierungen handelt, die neben empirischen Daten auch auf theoriegestützten Annahmen beruhen.

Für die Modellierung im GIS wurde die stufenlose Standardisierung der Konfliktpotenziale zwischen 0 und 1, wie sie in den Kartendarstellungen des Kapitels 3.4.3 vorgenommen wurde, auf eine diskrete Skala mit drei Werteklassen des Konfliktpotenzials umgerechnet. Hierfür wurde die Nutzungswahrscheinlichkeit der Indikatoraktivitäten linear in drei Werteabschnitte, d.h. in drei gleich große Skalenintervalle (hoch, mittel, gering), klassifiziert. Die Vorkommenswahrscheinlichkeit des Wildschweins beruht auf Angaben zu Lebensraumnutzung und Wildverteilung von Regionalexperten und wurde mit Hilfe revierbezogener Abschussdaten validiert; die Klassifikation in zwei Werteklassen (hoch, mittel) folgt grundsätzlich der Abstufung, wie sie bei der Erstellung der Verbreitungskarte in Kap. 3.4.2.2 (Abbildung 84) verwendet wurde. „Hohe“ Vorkommenswahrscheinlichkeit bezeichnet die Hauptlebensräume des Schwarzwilds; da diese Wildart im Wienerwald flächendeckend in vergleichsweise hoher Dichte auftritt, wurden alle anderen Flächen mit „mittlerer“ Vorkommensdichte eingestuft.

Durch die Kombination von drei Klassen der Nutzungswahrscheinlichkeit mit zwei Klassen der Vorkommenswahrscheinlichkeit des Wildschweins ergeben sich insgesamt sechs Kombinationsmöglichkeiten, die unterschiedlichen Ausprägungsformen des Konfliktpotenzials entsprechen. Jeder der sechs Konfliktpotenzialausprägungen wird ein eigener Farbwert zugewiesen. Die Kombination erfolgt über die Verknüpfungsmatrix in Abbildung 121 mittels der darin festgelegten Verknüpfungsregeln.

Die Karte in der nachstehenden Abbildung 120 zeigt die Lage und räumliche Verteilung der sechs Konfliktpotenzialstufen im Biosphärenpark. In den nachfolgenden Kapiteln werden den unter-schiedlichen Konfliktpotenzialausprägungen differenzierte Managementziele sowie besonders geeignete Maßnahmenoptionen für das Besuchermanagement und für weitere spezifische Landnutzergruppen zugeordnet. Über die Karte in Abbildung 120 ist näherungsweise die räumliche Zuordnung von vorrangigen Zielen und Maßnahmenoptionen des Konfliktmanagements zu Konflikträumen unterschiedlicher Konfliktpotenzialstufe möglich. 


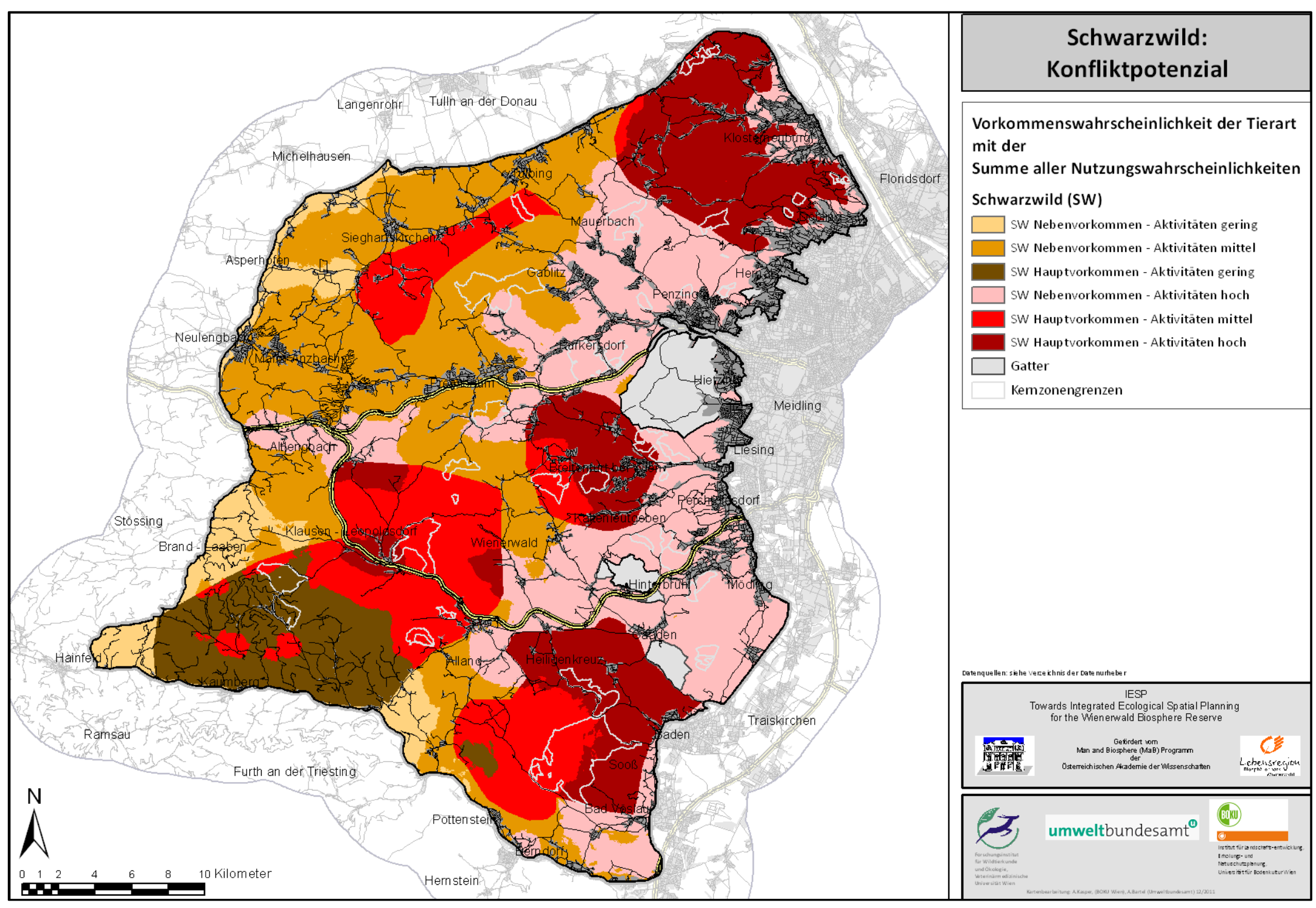

Abbildung 120: Räumliche Verteilung des Konfliktpotenzials zwischen dem Schwarzwild und der Summe aller Freizeitaktivitäten in sechs Werteklassen. 


\subsection{Verknüpfungsmatrix}

Mittels nachstehender Abbildung 121 wird die Vorkommenswahrscheinlichkeit des Wildschweins mit der summierten Nutzungswahrscheinlichkeit aller Indikator-Freizeitaktivitäten verknüpft. Über die aus der Abbildung ersichtlichen Vernüpfungsregeln ergeben sich sechs Kombinationsmöglichkeiten, die unterschiedlichen Ausprägungsformen bzw. Stufen des Konfliktpotenzials entsprechen. Die Verknüpfungsmatrix erleichtert die Zuordnung von Zielen für das Konfliktmanagement (siehe Kap. 4.3.2.3.4) sowie von besonders geeigneten Maßnahmenoptionen (siehe Kap. 4.3.2.3.5 und Kap. 4.3.2.3.7) zu unterschiedlichen Konflikt(potenzial)situationen. Über die zugewiesenen Farbwerte wird weiters die näherungsweise räumliche Zuordnung von prioritären Managementoptionen in der Karte in Abbildung 120 (Kap. 4.3.2.3.1) möglich.

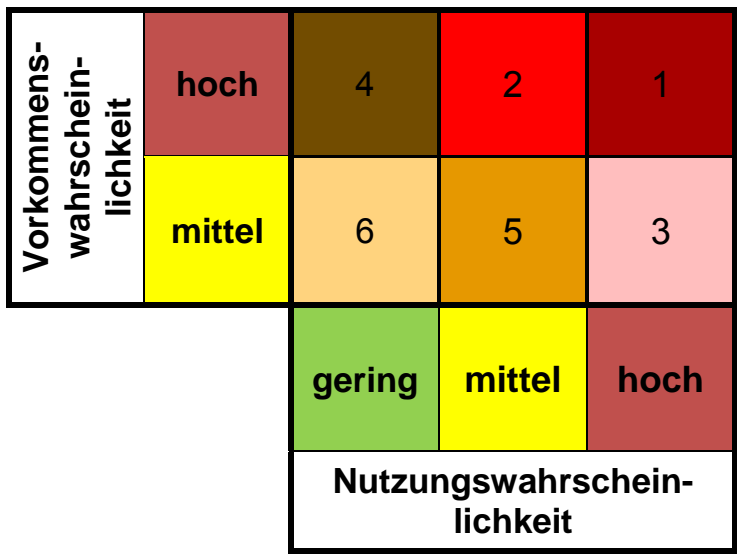

Ausprägung Konfliktpotenzial

\begin{tabular}{l|l|l|l|l|l|l|}
\hline gering & 6 & 5 & 4 & 3 & 2 & 1 \\
\hline
\end{tabular}

Abbildung 121: Verknüpfungsmatrix (oben) der Vorkommenswahrscheinlichkeit des Schwarzwilds mit der Summe der Nutzungswahrscheinlichkeiten aller Freizeitaktivitäten sowie Farbskala (unten) der Ausprägung des Konfliktpotenzials.

\section{Erläuterungen:}

Vorkommenswahrscheinlichkeit: Bezeichnet die Vorkommensdichte des Schwarzwilds im Biosphärenpark Wienerwald, d.h. die Wahrscheinlichkeit, dass auf einer Fläche Schwarzwild in hoher oder mittlerer Dichte auftritt. „Hohe“ Vorkommenswahrscheinlichkeit bezeichnet die Hauptlebensräume des Schwarzwilds; da diese Wildart im Wienerwald flächendeckend auftritt, wurden alle anderen Flächen mit „mittlerer" Vorkommensdichte eingestuft. Die räumliche Darstellung der Vorkommensdichte basiert auf lokalem Wissen von Regionalexperten. Die Vorkommenswahrscheinlichkeit wurde in zwei Klassen (hoch, mittel) klassifiziert und kann als Maß für die Sensibilität der betreffenden Fläche gegenüber Störungseinflüssen durch die Indikator-Erholungsaktivitäten interpretiert werden.

Nutzungswahrscheinlichkeit: Fungiert als Maß für die modellierte Nutzungsintensität aller Indikator-Erholungsaktivitäten. Es werden drei linear skalierte Werteklassen (hoch, mittel, gering) differenziert.Die Nutzungswahrscheinlichkeit ist als potenzielle Erholungsnachfrage auf Basis theoretischer Annahmen und von GIS-basierten Modellierungen zu verstehen. 
Konfliktpotenzial-Ausprägung bzw. Konfliktpotenzialstufe: Aus der Verknüpfung von zwei Skalenstufen der Vorkommenswahrscheinlichkeit mit drei Skalenstufen der Nutzungswahrscheinlichkeit ergeben sich im Fall des Wildschweins sechs unterschiedliche Konfliktpotenzialausprägungen. Jede Kombination von Vorkommens- und Nutzungswahrscheinlichkeit repräsentiert eine spezifische Konflikt(potenzial)situation, die wiederum jeweils spezifische Managementziele und spezifischen Managementbedarf erfordern kann. Unter "Managementbedarf" ist zu verstehen, dass auf Flächen unterschiedlicher Ausprägung von Konfliktpotenzialen jeweils unterschiedliche Maßnahmenoptionen in unterschiedlicher Kombination als besonders geeignet erscheinen können, um die vorrangigen Managementziele zu erreichen. Die Verknüpfungsmatrix erleichtert somit einerseits die Zuordnung von prioritären Maßnahmenoptionen zu bestimmten Konfliktsituationen und andererseits über die Farbcodes der Karte in Abbildung 120 die näherungsweise räumliche Zuordnung zu entsprechenden Konflikträumen. 


\subsection{Generelle Ziele für das Konfliktmanagement in Bezug auf das Wildschwein}

Das seit Jahrzehnten anhaltende Populationswachstum des Scharzwilds - mit tendenziell steigenden Wachstumsraten - hat auch in der Wienerwaldregion zu vermehrten Problemen geführt, die sich während der letzten Jahre zunehmend verschärft haben. Diese primär durch das Populationswachstum bedingte Dynamisierung der Problemlagen umfasst zunehmende Wildschäden durch das Schwarzwild an Agrar- und Weinkulturen, landwirtschaftlichem Grünland und naturschutzfachlich wertvollen Grünlandbiotopen. Zudem ist das Vordringen von Wildschweinen in das Siedlungs- und Stadtgebiet mit zunehmenden Wildschäden an Privatgärten und öffentlichen Grünanlagen verbunden und stellt ein Gefährdungspotential gegenüber Haustieren und Menschen dar.

Vor diesem Hintergrund lassen sich generelle Ziele in Bezug auf die Indikatorart Wildschwein (Schwarzwild) im Biosphärenpark Wienerwald insbesondere aus dem im Jagdrecht verankerten Auftrag zur Wildbewirtschaftung unter Rücksichtnahme auf die Interessen der Landund Forstwirtschaft ableiten. Übergeordnete Ziele für das Schwarzwild müssen sich im Wienerwald daher vor allem auf die Bestandesreduktion und die Wildschadenvermeidung im landeskulturellen sowie wirtschaftlichen Interesse beziehen. Konfliktpotenziale zwischen dem Schwarzwild und Freizeitaktivitäten liegen daher insbesondere dann vor, wenn die Erfüllung der beiden Hauptziele Bestandesregulation und Wildschadenvermeidung gefährdet wird. Entscheidende, durch Freizeitbetrieb im Wildlebensraum ausgelöste Wirkungsmechanismen stellen dabei die Störung des Raum-Zeit-Verhaltens des Schwarzwilds, die Störung des Jagdbetriebs, d. h. der jagdlichen Abschusserfüllung, sowie die Verdrängung von Schwarzwild in wildschadenanfällige Agrarbereiche und in jagdlich schwer behandelbare Siedlungsgebiete dar. Als kritische Einflussgrößen im Gefüge der Wechselwirkungen zwischen Schwarzwildpopulationen und Freizeitaktivitäten lassen sich daher die Bejagbarkeit des Schwarzwildes, die Auslösung oder Verschärfung von Wildschäden und Verdrängungseffekte in problematische Ausweichhabitate identifizieren.

Im Hinblick auf die Steuerung von Konfliktpotenzialen zwischen Freizeit- und Erholungsnutzungen einerseits und dem Schwarzwild andererseits sind in der biosphärenparkweiten Betrachtung vor allem folgende übergeordnete Ziele besonders maßgeblich:

- Wirksame Kontrolle und Regulation der Schwarzwildbestände auf ein langfristig landeskulturell und ökonomisch tragfähiges Niveau

- Reduktion und Vermeidung von Wildschäden durch Schwarzwild an landwirtschaftlichen Kulturen sowie naturschutzfachlich wertvollen Biotopen

- Vermeidung einer verstärkten Nutzung von Siedlungsgebieten durch das Schwarzwild

- Vermeidung von Störungen, die die Bejagbarkeit und Abschusserfüllung verschlechtern und die Entstehung von Wildschäden begünstigen 


\subsection{Wildschwein: Zuordnung von Leitfunktionen und Managementzielen zu Kon- fliktpotenzialstufen}

In der nachstehenden Tabelle 46 werden prioritäre Leitfunktionen und Zielausrichtungen für das Konfliktmanagement in Bezug auf die Indikatorart Wildschwein nach Ausprägungsformen des Konfliktpotenzials differenziert. Die Priorisierungen und Zuweisungen von Leitfunktionen und spezifischen Managementzielen spiegeln den unterschiedlichen Managementbedarf je nach Konfliktpotenzialstufe wider und sind als Orientierungshilfe für die nachfolgende Auswahl besonders geeigneter Managementoptionen zu verstehen.

Tabelle 46: Zuordnung von Leitfunktionen und Managementzielen zu Konfliktpotenzialausprägungen für das Wildschwein.

\begin{tabular}{|l||l||l|l|}
\hline \multicolumn{2}{|l|}{ WILDSCHWEIN: } \\
Zuordnung von Leitfunktionen und Managementzielen zu Konfliktpotenzialausprägungen \\
\hline \hline
\end{tabular}




\begin{tabular}{|c|c|c|c|c|}
\hline 3 & mittel & hoch & $\begin{array}{l}\text { Eignungsbereich für } \\
\text { kleinräumige Kom- } \\
\text { pensations- bzw. } \\
\text { Alternativangebote } \\
\text { zur Besucherlen- } \\
\text { kung }\end{array}$ & $\begin{array}{l}\text { - Potenzieller Eignungsbereich für punktuelle } \\
\text { Atttraktivierungsangebote für Freizeitnutzer, um } \\
\text { Schwarzwild-Hauptlebensräume andernorts zu beruhi- } \\
\text { gen bzw. ruhig zu halten } \\
\text { - Prüfung räumlich-zeitlich begrenzter Nutzungsrestriktio- } \\
\text { nen für Freizeitbetrieb in Wildschadensgebieten, um in- } \\
\text { tensivierte Bejagung zu ermöglichen und } \\
\text { Bestandeszunahmen zu vermeiden } \\
\text { - Enge Maßnahmenabstimmung zwischen Besucherma- } \\
\text { nagement und Jagd, um landwirtschaftliche Wildschä- } \\
\text { den, Verdrängung von Schwarzwild ins Siedlungsgebiet } \\
\text { sowie Bestandeszunahme zu vermeiden } \\
\end{array}$ \\
\hline 4 & hoch & gering & $\begin{array}{l}\text { Regulation } \\
\text { Schwarzwild - } \\
\text { Erhaltung geringer } \\
\text { Störungsintensität } \\
\text { (beruhigt halten) }\end{array}$ & $\begin{array}{l}\text { - Geringhalten der Erholungsnachfrage durch Besucher- } \\
\text { lenkungsmaßnahmen, mit Präferenz für „,sanfte“ Maß- } \\
\text { nahmen und Anreizen für Besucher in weniger sensib- } \\
\text { len Gebieten andernorts } \\
\text { - Wirksame jagdliche Bestandesregulation } \\
\text { - Intensivierte Bejagung in Wildschadensgebieten }\end{array}$ \\
\hline 5 & mittel & mittel & $\begin{array}{l}\text { Vorsichtsbereich - } \\
\text { Vermeidung von } \\
\text { Verschlechterungen }\end{array}$ & $\begin{array}{l}\text { - Zunahme der Erholungsnachfrage vermeiden } \\
\text { - Bestandeszunahme des Schwarzwildes verhindern }\end{array}$ \\
\hline 6 & mittel & gering & $\begin{array}{l}\text { Erhaltung Status } \\
\text { quo }\end{array}$ & $\begin{array}{l}\text { - Effektive Umsetzung der jagdlichen Bestandesreduktion } \\
\text { des Schwarzwilds } \\
\text { - Keine Einschränkungen für Freizeitbetrieb notwendig } \\
\text { - Erhaltung störungsarmen Naturraums im Sinne generel- } \\
\text { ler Nachhaltigkeitsziele des Biosphärenparks }\end{array}$ \\
\hline
\end{tabular}




\subsection{Wildschwein: Übersicht über Handlungsoptionen des Besuchermanage- ments und querschnittsorientierte Maßnahmenoptionen nach Konfliktpoten- zialstufen}

In der nachstehenden Tabelle 47 werden die in Kap. 4.2 beschriebenen Maßnahmenoptionen aus den Bereichen Besuchermanagement und querschnittsorientierte Maßnahmen den Konfliktpotenzialstufen in Bezug auf das Wildschwein zugeordnet. Die Nummerierung der Maßnahmen entspricht der Gliederung in Kap. 4.2. Die Zordnung gibt Hinweise darauf, welche Maßnahmen grundsätzlich besonders geeignet erscheinen, um in unterschiedlichen Konfliktpotenzialsituationen zum Konfliktmanagement beizutragen. Die unterschiedlichen Konfliktpotenzialstufen entsprechen den farblich differenzierten Konfliktpotenzialräumen in Abbildung 120. Die Tabelle ist als Entscheidungshilfe für die Maßnahmenauswahl und priorisierung zu verstehen und sollte im Sinne eines Maßnahmenbaukastens benutzt werden. 
Tabelle 47: Besonders geeignete Maßnahmenoptionen (Besuchermanagement, Querschnittsmaßnahmen) je Konfliktpotenzialstufe für das Wildschwein

\begin{tabular}{|c|c|c|c|c|c|c|c|}
\hline \multicolumn{8}{|c|}{$\begin{array}{l}\text { WILDSCHWEIN } \\
\text { Zuordnung von Maßnahmenpräferenzen (Besuchermanagement, Querschnittsmaßnahmen) zu den Konfliktpotenzialausprägungen }\end{array}$} \\
\hline \multicolumn{2}{|c|}{ Konfliktpotenzialstufe } & $\overline{1}$ & $\overline{2}$ & 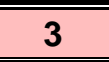 & 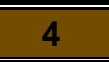 & $\overline{\overline{5}}$ & (ب6 \\
\hline & Vorkommenswahrscheinlichkeit (Sensibilität) & hoch & hoch & mittel & hoch & mittel & mittel \\
\hline & Nutzungswahrscheinlichkeit (Erholungsnachfrage) & hoch & mittel & hoch & gering & mittel & gering \\
\hline \multicolumn{8}{|c|}{ Maßnahmenoptionen } \\
\hline Nr. ${ }^{*}$ & Bezeichnung & & & & & & \\
\hline \multicolumn{8}{|c|}{ Besuchermanagement - Freizeit- und Erholungsaktivitäten } \\
\hline \multicolumn{8}{|c|}{ Kleinräumige Maßnahmenoptionen } \\
\hline $\boldsymbol{A}$ & Räumlich-zeitliche Nutzungsrestriktionen & & & & & & \\
\hline A1 & Vereinheitlichung rechtlich verankerter Nutzungsverbote und -gebote (Kernzonen) & $\mathbf{X}$ & $\mathbf{x}$ & $\mathbf{x}$ & $\mathbf{x}$ & $\mathbf{x}$ & $\mathbf{x}$ \\
\hline A2 & Verzicht auf Ausbau der Wegeinfrastruktur & $\mathbf{x}$ & $\mathrm{x}$ & & $\mathbf{x}$ & $\mathbf{x}$ & $\mathbf{x}$ \\
\hline A3 & Verlegung bzw. Auflassung von Wegen und kernzonengerechtes Wegenetz & $(\mathrm{X})$ & & & & & \\
\hline A4 & $\begin{array}{l}\text { Freiwillige Vereinbarungen zu örtlich begrenzten, räumlichen und/oder zeitlichen Nut- } \\
\text { zungseinschränkungen }\end{array}$ & $\mathrm{x}$ & $(\mathrm{X})$ & $(\mathrm{X})$ & & & \\
\hline A5 & $\begin{array}{l}\text { Jagdrechtlich begründete Nutzungsrestriktionen (Wildschutzgebiete, befristete jagdli- } \\
\text { che Sperrgebiete) }\end{array}$ & $\mathbf{x}$ & $\mathrm{x}$ & $(\mathrm{X})$ & & & \\
\hline B & Besucherlenkung durch De-Attraktivierung & & & & & & \\
\hline B1 & Landschaftsgestalterische Maßnahmen zur Verringerung der Zugänglichkeit & $\mathbf{x}$ & $\mathbf{x}$ & & $\mathbf{x}$ & $(\mathrm{X})$ & \\
\hline B2 & Verzicht auf Schneeräumung & $\mathbf{x}$ & $\mathbf{x}$ & & $\mathbf{x}$ & $(\mathrm{X})$ & \\
\hline B3 & Management von Infrastruktur mit Schleusenfunktion & (X) & & & & & \\
\hline C & Kleinräumige Kompensations- bzw. Alternativangebote zur Besucherlenkung & & & & & & \\
\hline $\mathrm{C} 1$ & Hundeauslaufbereiche & & & $\mathbf{x}$ & & & \\
\hline $\mathrm{C} 2$ & Night Trails für Jogger & & & $\mathbf{x}$ & & & \\
\hline $\mathrm{C} 3$ & Night Trails und Winter Trails für Mountainbiker & & & $\mathrm{x}$ & & & \\
\hline C4 & Mountainbike Technik Trails & & & $\mathbf{x}$ & & & \\
\hline C5 & Mountainbike Downhill-Strecken & & & $\mathrm{x}$ & & & \\
\hline C6 & Betreute Picknickplätze & & & $\mathrm{X}$ & & & \\
\hline C7 & Wildlife Viewing-Einrichtungen (Schwarzstorch-Beobachungswarten) & & & & & & \\
\hline $\mathrm{C} 8$ & Verstärkte Instandhaltung und Pflege der bestehenden Wegeinfrastruktur & & & $\mathbf{x}$ & & & \\
\hline D & Verstärkte Information, Kontrolle und Vollzug in Schwerpunktbereichen & & & & & & \\
\hline
\end{tabular}




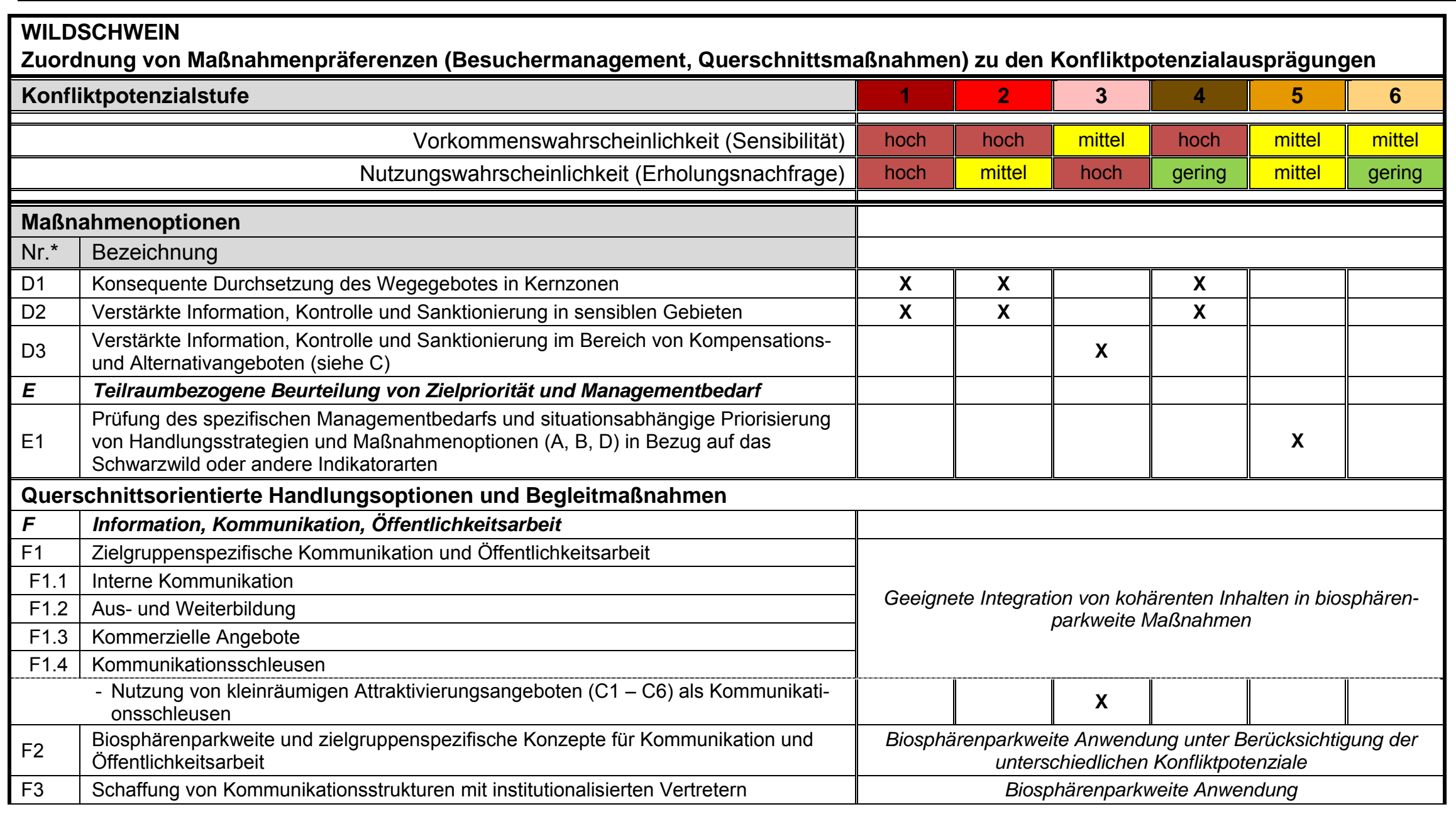




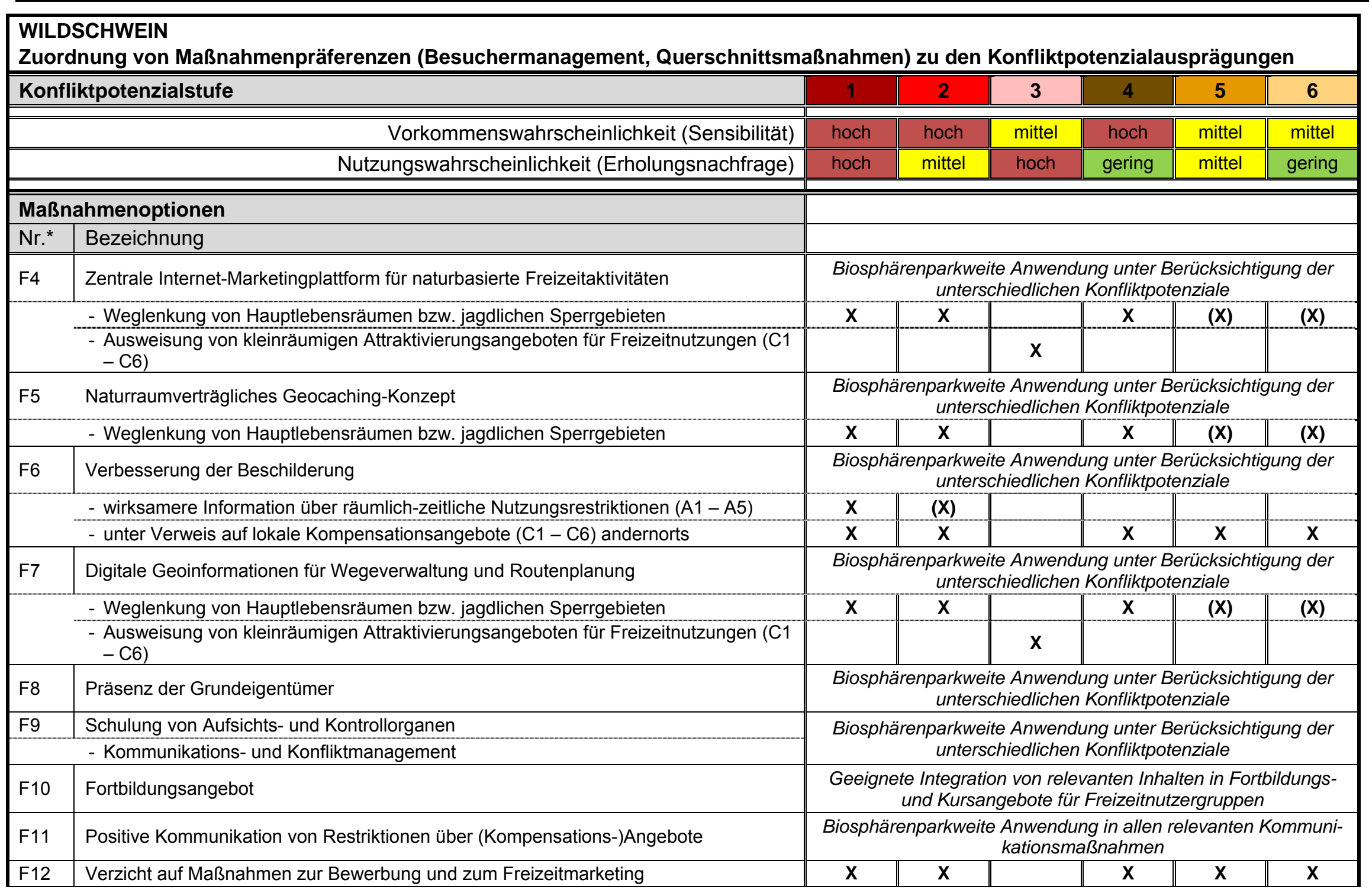




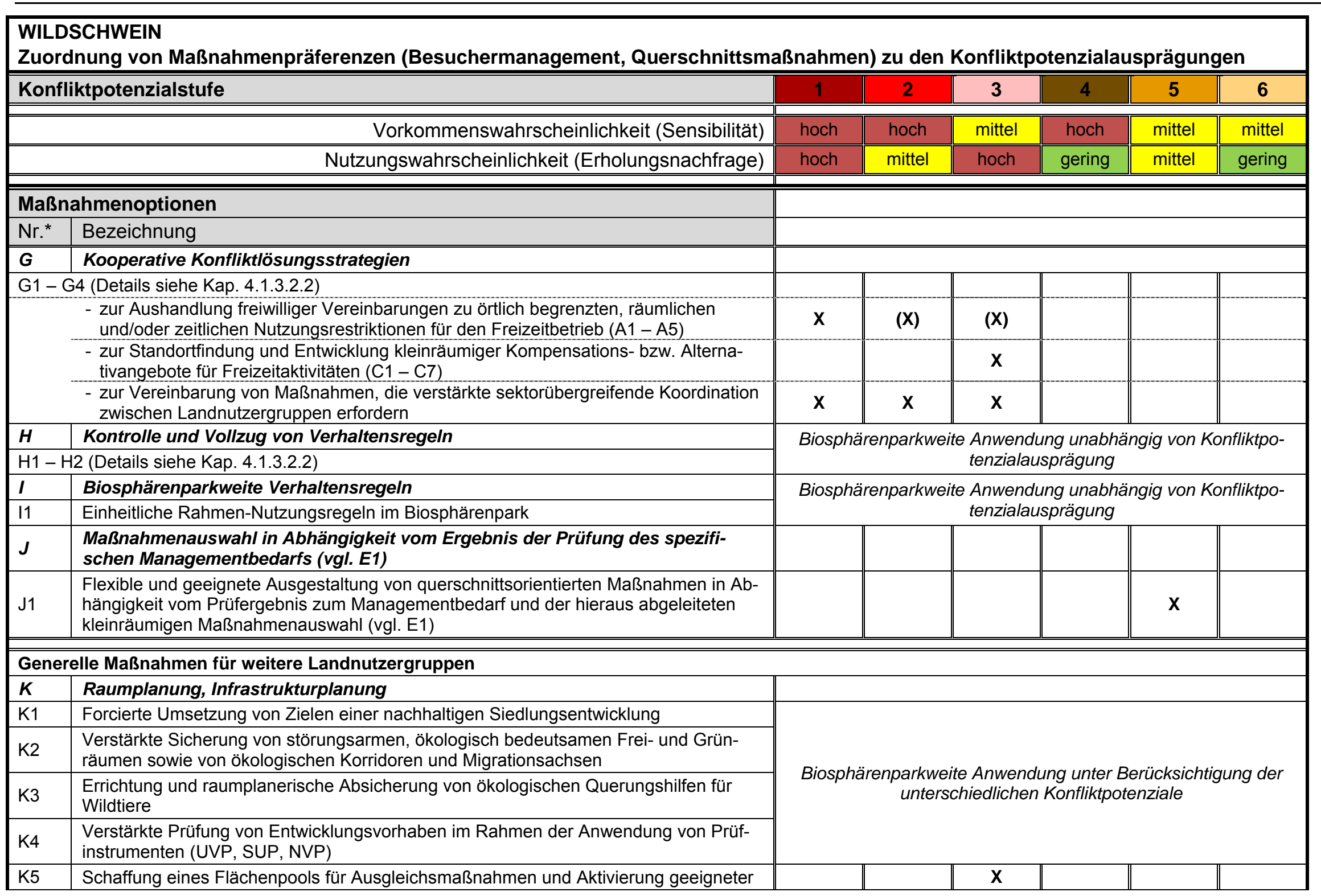

IESP - Towards Integrated Ecological Spatial Planning for the Wienerwald Biosphere Reserve 


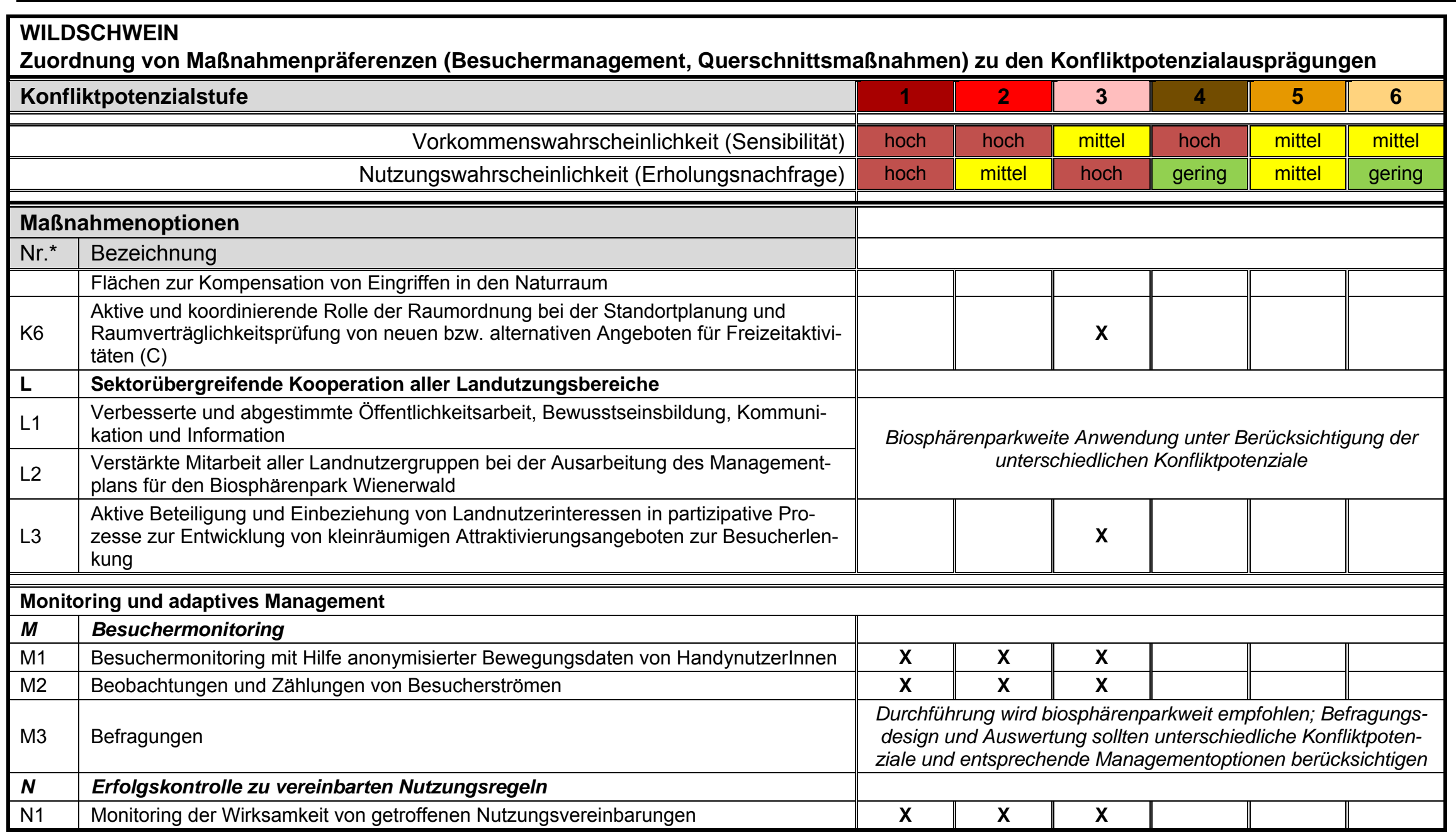

Zeichenerklärung: $\quad$ X ... prioritäre Managementoption; $(\mathbf{X})$... mit Vorbehalt geeignete bzw. weniger prioritäre Managementoption 


\subsection{Spezifische Maßnahmenoptionen für weitere Landnutzergruppen in Bezug auf das Wildschwein}

\section{$\underline{\text { Jagdwirtschaft }}$}

Erhöhung der Schwarzwildabschüsse und der Bejagungseffizienz: Grundsätzlich handelt es sich beim Schwarzwild im Wienerwald, wie auch in den meisten anderen Regionen Mitteleuropas, um eine Wildart mit landeskulturellem Reduktionsbedarf. Das Ziel der effektiven Bestandesreduktion bzw. -regulation gilt daher in allen Teilgebieten des Biosphärenparks gleichermaßen, d. h. unabhängig von der jeweiligen Ausprägung des Konfliktpotenzials mit Freizeitaktivitäten. Die konsequente Ausrichtung der Bejagungsstrategie und des Bejagungsmanagements auf die Abschusserhöhung sowie die Erhöhung der Bejagungseffizienz auf regionaler (und überregionaler) Ebene ist auch deswegen notwendig, um den „Export“ von Populationsüberschüssen in angrenzende Regionen sowie räumliche Ausweich- und Verdrängungseffekte zu verhindern. Je nach vorherrschender Intensität von Beunruhigungen durch Freizeitnutzungen sowie nach der Wildschadensanfälligkeit von landwirtschaftlichen Kulturen bzw. der Lage von aktuellen Wildschadensschwerpunkten können die Herausforderungen an das Bejagungsmanagement jedoch stark unterschiedlich sein, d. h. unterschiedliche Bejagungsstrategien und Jagdmethoden erfordern. So sind beispielsweise revierübergreifende Bewegungsjagden in Gebieten mit hoher Freizeitnutzungsintensität (ohne befristete Nutzungsrestriktionen für Freizeitnutzer) nur schwer durchführbar. Das Ziel der Erhöhung der Bejagungseffizienz bedeutet im Wesentlichen, mehr Abschüsse mit weniger Jagdtagen zu erzielen. Dies ist insbesondere auch deshalb notwendig, um zusätzliche jagdliche Beunruhigungen des Schwarzwildes durch zu hohen Jagddruck zu minimieren.

Im Regelfall bietet die Intervalljagd mit ausreichenden Jagdruhephasen - auch während der Zeit des Regulationsabschusses im Winter - eine gut geeignete jagdtechnische Option, weil hierdurch unnötiger Jagddruck vermieden und die effiziente Bejagbarkeit des Schwarzwildes erhalten werden kann. Bei einer umsichtigen Planung sind so nach einer längeren jagdlichen Ruhephase deutlich höhere Abschüse zu erwarten, als es bei über das Jahr konstantem Jagddruck möglich wäre.

Für die erforderliche Regulierung des Schwarzwildes kann in manchen Gebieten auf die Jagd in der Nacht (z. B. an Kirrstellen) nicht verzichtet werden; dies gilt vor allem für Wildschadensgebiete mit Schwerpunktbejagung. Andererseits ist zu berücksichtigen, dass die Wildbejagung in der Nacht zur zusätzlichen Beunruhigung des Wildes auch während der Nachtstunden führt, wodurch Raumnutzung und Ernährungsryhthmus des Wildes (auch anderer als der bejagten Wildarten) gestört und auch Wildschäden an der Vegetation sowie in Siedlungsgebieten ausgelöst werden können. Bei häufiger Nachtjagd wird auch die Bejagbarkeit des Schwarzwildes erschwert, bedingt durch die erhöhte Scheuheut des Wildes. Daher sollte die Nachtjagd auf Schwarzwild außerhalb von Schwerpunktbejagungsgebieten grundsätzlich zurückhaltend angewandt und die Zahl der Nachtjagdtage limitiert werden. In Gebieten mit Vorkommen besonders störungssensibler Wildarten, wie dem Rotwild, sowie in siedlungsfernen Jagdgebieten mit geringer Freizeitnutzungsintensität, wo Bewegungsjagden untertags leichter durchzuführen sind, ist hingegen ein völliger Verzicht auf die Bejagung in der Nacht oder eine starke Limitierung zu empfehlen (vgl. Reimoser et al., 2008).

Intensivierte Schwerpunktbejagung in Wildschadensgebieten: In Wildschadensgebieten sowie in Gebieten mit erhöhter Wildschadenanfälligkeit, d.h. mit hohem Feldanteil und hohem Anteil schadensanfälliger Kulturen, ist die Intensivierung der Bejagung zur Bestandeskontrolle sowie zur Vergrämung von Wildschweinen von den schadensanfälli- 
gen Flächen erforderlich. Insbesondere in Hauptlebensräumen des Schwarzwildes mit erhöhter Vorkommensdichte ist darauf zu achten, dass Schwerpunktbejagungen auf die unmittelbaren Schadensgebiete konzentriert bleiben, um das Schwarzwild nicht in noch verbliebenen störungsarmen Einständen zu beunruhigen, die Bejagbarkeit auf der Restfläche zu erhalten und Verdrängungseffekte in angrenzende schadensanfällige oder jagdlich schwer behandelbare Gebiete zu vermeiden. Schwerpunktbejagungen in Schadens(risiko)gebieten mit gleichzeitig hoher Freizeitnutzungsintensität stellen die Jagd vor besonders große Herausforderungen. In solchen Teilräumen kann es spezifischer Besucherlenkungsmaßnahmen, z. B. im Rahmen von freiwilligen Vereinbarungen zu (befristeten) räumlich-zeitlichen Nutzungsrestriktionen oder im Rahmen von befristeten jagdlichen Sperrgebieten, bedürfen, um den Bejagungserfolg und die Sicherheit der Schussabgabe zu gewährleisten. In diesen Fällen ist die Maßnahmenabstimmung mit den betreffenden Freizeitnutzergruppen besonders wichtig.

Ausrichtung der Bejagungsstrategie an der Wildschadenanfälligkeit von landwirtschaftlichen Kulturen: Grundsätzlich sollte das Schwarzwild im Sommer überwiegend im landwirtschaftlichen Offenland bejagt werden, um die Wildschweine durch den Jagddruck im Waldlebensraum nicht aus den dortigen Einständen in die Deckung von Feldkulturen zu treiben, wo wiederum unvermeidlich zusätzliche Schäden entstehen würden. Im Winter bzw. nach dem Abernten schadensgefährdeter Feldfrüchte kann der Schwerpunkt der jagdlichen Bestandeskontrolle dann verstärkt in den Wald verlagert werden. Auch während dieser Zeit sollten ausreichende Jagdruhephasen eingehalten werden.

Erhaltung störungsberuhigter Einstandsräume des Schwarzwilds: In Hauptlebensräumen des Schwarzwilds, wo trotz hoher Erholungsnachfrage hohe Vorkommensdichten bestehen, kann davon ausgegangen werden, dass noch funktionierende, störungsarme Einstands- und Rückzugsräume vorhanden sind. Sofern diese nicht schadensanfällig sind, sollten sie grundsätzlich erhalten bleiben, d.h. auch von jagdlicher Seite von intensiven Bejagungen ausgespart bleiben. Jede Schwarzwildbejagung in solchen störungsberuhigten Habitaten sollte jedenfalls das Risiko berücksichtigen, dass die Tiere durch den Jagddruck in andere, unter Umständen schadensanfällige Gebiete verdrängt werden.

Revierübergreifende Bejagungstrategie: Gerade bei einer großräumig agierenden und nahezu flächendeckend in hoher Dichte vorkommenden Wildart wie dem Schwarzwild ist eine revierübergreifende Abstimmung der Bejagung besonders erforderlich. Dieser Bedarf wird durch die im österreichweiten Vergleich geringen durchschnittlichen Reviergrößen in weiten Teilen des Wienerwalds verstärkt. Insbesondere Bewegungsjagden erfordern eine revierübergreifende Vorgangsweise. Darüber hinaus wäre eine biosphärenparkweit abgestimmtes Schwarzwild-Bejagungskonzept anzustreben. Ohne großräumige Abstimmung der Schwarzwildbejagung besteht stets ein erhebliches Risiko, dass die Tiere infolge örtlichen Jagddrucks ihren Aufenthaltsschwerpunkt verlagern, und dies womöglich in schadensanfällige oder jagdlich schwer behandelbare Räume.

Restriktive Handhabung der Kirrung: Kirrungen sind zur Effizienzsteigerung der Abschusserfüllung bei der Schwarzwild-Bejagung vielfach unverzichtbar. Um zu vermeiden, dass die Kirrung einen zusätzlichen Beitrag zur Ernährung des Wildes darstellt und somit das Populationswachstum der Schwarzwildbestände weiter verstärkt, ist es wesentlich, dass die Futtervorlage nach Menge, Ort und Zeitraum limitiert erfolgt und ausschließlich dem Zweck der Abschusserleichterung dient (vgl. Reimoser et al., 2008).

Vereinbarung definierter Jagdtage bzw. -zeiten mit anderen Landnutzergruppen: Vor allem in Gebieten mit hoher Vorkommensdichte und gleichzeitig hoher Freizeitnutzungsintensität, in Siedlungsnahlage (Stadtrandgebiet) sowie in ansonsten schwer zu be- 
jagenden Wildschadenschwerpunktgebieten kann es vorteilhaft sein, die Festlegung definierter Jagdtage bzw. Jagdzeiten mit Vertretern von Freizeit- und Erholungsnutzergruppen, Gemeinden und Grundeigentümern anzustreben. Derartige Vereinbarungen müssen im Rahmen von kooperativen Konfliktlösungsmodellen (vgl. Kap. 4.2.1.2, Maßnahmengruppe G) ausgehandelt werden und wären als freiwilliges „gentleman's agreement" zu betrachten. Während solcher definierten Jagdtage bzw. -tageszeiten sollten Freizeitaktivitäten in den betreffenden Bereichen weitest möglich unterbleiben. Diese Maßnahme würde eine effiziente jagdliche Abschusserfüllung bei geringem Jagddruck während des restlichen Jahres ermöglichen und die Sicherheit der Schussabgabe unterstützen.

Zeitlich befristete Jagdsperrgebiete: Das Niederösterreichische Jagdgesetz sieht die Möglichkeit der Einrichtung von jagdlichen Sperrgebieten vor. Dieses jagdrechtliche Instrument ist insbesondere zu Durchführung von Bewegungsjagden vorgesehen. Vor allem in Gebieten mit hoher Freizeitnutzungsintensität sowie in Siedlungsrandlagen, wo die Tendenz zur Abwanderung des Schwarzwilds in das Siedlungsgebiet besteht, ermöglichen es Jagdsperrgebiete, den Regulationsabschuss effektiv durchzuführen bzw. Wildschadensgebiete schwerpunktmäßig im öffentlichen Interesse zu bejagen, ohne die Sicherheit von jagdfremden Personen zu gefährden (vgl. auch Empfehlung A5 in Kap. 4.2.1.1). Gerade im Stadtrandgebiet von Wien, wo zuletzt teils massive Wildschäden an Agrar- und Weinkulturen sowie vermehrtes Eindringen von Wildschweinen in Privatgärten und in bebautes Gebiet zu beobachten waren, kann diese Option verstärkt in Betracht gezogen werden. Wesentlich dabei erscheint in jedem Fall eine enge und klare zeitliche Befristung der Maßnahme sowie eine konsensorientierte Vorgangsweise und offene Kommunikation gegenüber anderen Nutzergruppen im Wildlebensraum.

Abschussvorgaben in Jagdpachtverträgen: Bislang sind für das Schwarzwild keine Abschusspläne im niederösterreichischen und Wiener Jagdgesetz vorgesehen. Um dem Reduktionsbedarf dieser Wildart Rechnung zu tragen, sollten jagdberechtigte Grundeigentümer in ihrer Rolle als Jagdverpächter vermehrt von ihrer Möglichkeit Gebrauch machen, abseits von behördlichen Abschussvorgaben Mindestabschüsse im Rahmen von (schriftlichen oder mündlichen) vertraglichen Vereinbarungen mit Jagdpächtern oder längerfristigen Jagdkunden festzulegen. Eine Zielvorgabe könnte z. B. auch sein, über die Zielwerte (Mindestabschüsse) hinaus alle abschussmöglichen Wildschweine zu erlegen (vgl. Reimoser et al., 2008).

\begin{abstract}
Aktive Beteiligung an partizipativen Prozesse zur Entwicklung von kleinräumigen Angebotsschaffungen zur Besucherlenkung: Durch aktive Beteiligung von Jagdvertretern sollten legitime Interessen von Jagdberechtigten und Jagdausübungsberechtigten in Prozesse zur Entwicklung von kleinräumigen Kompensations- bzw. Alternativangeboten für Freizeitnutzergruppen eingebracht werden. Mögliche negative Auswirkungen solcher Vorhaben auf Jagdwirtschaft und Wildökologie können so bestmöglich vermieden bzw. ausgeglichen werden. Zudem muss das Bejagungsmanagement im Umfeld betreffender neuer Freizeitstandorte entsprechend angepasst werden. Gerade im Hinblick auf das Schwarzwild ist in den für kleinräumige Attraktivierungsangebote geeigneten Teilräumen die Berücksichtigung der Wildschadenanfälligkeit des Gebiets erforderlich.
\end{abstract}

\title{
Forstwirtschaft
}

Schaffung effizienter Bejagungsmöglichkeiten im Wald: Der forstlichen Berücksichtigung der Wildbejagungsmöglichkeit kommt wesentliche Bedeutung bei der Regulation der Schwarzwildbestände - und damit in weiterer Folge auch bei der Vermeidung von Wildschäden an landwirtschaftlichen Kulturen - zu. Die Waldstruktur bestimmt die Sichtbarkeit 
des Wildes und damit die Abschussmöglichkeiten und die Bejagungseffizienz. So ist beispielsweie in dichten Beständen mit Pflegerückständen die Bejagbarkeit des Schwarzwildes stark erschwert, was eine höherere Bejagungsfrequenz mit größerem Jagddruck erforderlich macht, um zum selben Abschusserfolg zu gelangen. Die Anlage von Schussschneisen und Schussflächen durch Jagdausübungsberechtigte erfordert in der Regel die Zustimmung des Grundeigentümers. Da mit Anlage und Erhaltung dieser Flächen zumeist Eingriffe in die Waldvegetation erforderlich sind (Fällen von Bäumen, regelmäßiges Freischneiden zum Offenhalten), ist eine Abstimmung mit der forstlichen Maßnahmenplanung zielführend. Ausreichende Bejagungsmöglichkeiten tragen zur Wildbestandsregulierung bei und vermögen gleichzeitig den Jagddruck zu vermindern; beides wirkt sich positiv auf die Verminderung von Wildschaden aus. Wesentlich ist aber gerade in einem Biosphärenpark, dass Bejagungsflächen nur in dem Ausmaß eigens angelegt werden, als sie für eine effiziente Bejagung im Sinne der Wildstandsregulation und Abschussplanerfüllung sowie zur Erhaltung des Jagdwertes eines Reviers notwendig sind. Wo immer möglich, sollten anstelle eigens angelegter Schussschneisen durch forstliche Nutzungen entstehende Hiebsflächen und Räumungsflächen als Bejagungsflächen genutzt werden. Diese im „Kielwasser der forstlichen Nutzung“ erhöhte Bejagungseffizienz kann durch Abstimmung zwischen Waldeigentümern /-bewirtschaftern und Jagdausübenden optimiert werden. Zum Teil kann ein geringeres Ausmaß an vorhandenen Jagdflächen auch durch angepasste Bejagungsstrategien und Jagdtechniken kompensiert werden.

Grundsätzlich können Waldeigentümer bzw. -bewirtschafter zur Bestandesregulation des Schwarzwildes beitragen, indem ausreichend Bejagungsflächen (eigens angelegte Schussschneisen und -flächen, im Zuge der forstlichen Nutzung entstehende Flächen) ermöglicht werden. In Bezug auf die Kernzonen des Biosphärenparks sollten Eingriffe in die Waldstruktur, die der Jagdausübung dienen, allerdings möglichst auf Bereiche außerhalb der Kernzonengrenzen verlagert werden. Für die Pflege bestehender Schussschneisen bzw. die Schussfeldpflege (z. B. Entfernen einzelner überhängender Äste) innerhalb von Kernzonen wurden eigene Richtlinien erarbeitet, die auf die ökologischen Prozessschutzziele in Kernzonen besondere Rücksicht nehmen (Oitzinger et al, 2007; Reimoser et al., 2008). Die Neuanlage von Schussschneisen in Kernzonen sollte nur in begründeten Ausnahmefällen möglich sein und in den Kernzonen-Managementplänen verankert werden.

Forstliche Beiträge zur Vermeidung von Wildschäden an landwirtschaftlichen Kulturen: Durch planvolle und auf die Wildschadenanfälligkeit nahe liegender landwirtschaftlicher Flächen abgestimmte Anlage bzw. Erhaltung von Äsungsflächen für das Schwarzwild innerhalb von Waldlebensräumen, wie Waldwiesen und Wildäcker, kann das Schwarzwild gezielt von schadensanfälligen Ackerkulturen abgelenkt und im Wald gehalten werden. Wesentlich hierbei ist der gezielte Einsatz von Äsungsflächen zur Wildlenkung und Wildschadenvermeidung, ohne hierdurch das insgesamt für das Schwarzwild verfügbare Nahrungsangebot gravierend zu erhöhen. Um die gewünschte Lenkungswirkung erzielen zu können, erscheint eine enge forstliche, jagdliche und landwirtschaftliche Maßnahmenabstimmung unabdingbar. Auch kann die Forstwirtschaft darauf achten, keine Mastbäume unmittelbar an der Grenzlinie zwischen Waldrand und größerflächigen Ackerschlägen aufkommen zu lassen bzw. sollte die Begründung neuer Eichen- und Buchenbeständen unmittelbar angrenzend an große Ackerflächen vermieden werden.

\section{Landwirtschaft}

Landwirtschaftliche Maßnahmen zur Verringerung der Wildschadenanfälligkeit von Agrarkulturen: Eine landwirtschaftliche Einflussmöglichkeit auf die Wildschadenanfällig- 
keit besteht in der Wahl der Anbaukultur. Manche Feldfrüchte sind für das Schwarzwild deutlich attraktiver und daher schadensanfälliger als andere. So erhöht ein vermehrter Anbau von Mais deutlich das Wildschadenrisiko. Auf Grünland kann die Ausbringung von organischer Düngung (Bemistung) eine Lockwirkung auf Schwarzwild ausüben und das Umbrechen von Wiesen durch Wildschweine auslösen. Eine maßgebliche Handlungsmöglichkeit der Landwirtschaft zur Absenkung des Wildschadensrisikos besteht darin, möglichst wenig Besiedlungsanreize für das Schwarzwild zu schaffen. In diesem Zusammenhang sollte auf das Ackern bis zum Waldrand bzw. den Anbau von für das Schwarzwild attraktiven Feldfrüchten in Waldrandnähe verzichtet werden. Auch sollte darauf geachtet werden, dass in der Nähe von Eichen- und Buchenwaldbeständen kein zusätzlicher Besiedlungsanreiz auf angrenzendem Ackerland geschaffen wird, weil dies vor allem in Nicht-Mastjahren zu verstärkten Wildschäden im Feld führen kann.

Verbesserung der Bejagbarkeit von landwirtschaftlichen Offenlandflächen: Um die Bejagbarkeit von landwirtschaftlichen Kulturflächen zu verbessern, sollte der Anbau von Ackerkulturen bis unmittelbar an den Waldrand vermieden und stattdessen Schussschneisen freigehalten werden. Insbesondere auf groß parzellierten, gleichförmigen Ackerschlägen sollten mehrfach ausreichend breite Schussschneisen oder Schussflächen innerhalb der Kulturen angelegt werden, um das Ausweichen der Tiere vor den Bejagungsversuchen innerhalb des Deckung bietenden Aufwuchses zu verhindern.

\section{Alle Nutzergruppen}

Betreffend die aktive Beteiligung aller Landnutzergruppen an partizipativen Prozessen zur Entwicklung von kleinräumigen Kompensations- bzw. Alternativangeboten für Freizeitnutzergruppen (vgl. Kap. 4.2.2, Empfehlung L3) siehe die Übersichtsmatrix in Tabelle 47 (Kap. 4.3.2.3.5). 


\subsection{Wildschwein: Übersicht über spezifische Maßnahmenoptionen für weitere Landnutzergruppen nach Konfliktpotenzialstufen}

In der nachstehenden Tabelle 48 werden die im vorangehenden Kap. 4.3.2.3.6 beschriebenen Maßnahmenoptionen für einzelne Landnutzergruppen sowie die in Kap. 4.2.3 beschriebenen Maßnahmen zum Monitoring von Wildtierpopulationen und -lebensräumen den Konfliktpotenzialstufen in Bezug auf das Wildschwein zugeordnet. Die Zordnung gibt Hinweise darauf, welche Maßnahmen grundsätzlich besonders geeignet erscheinen, um in unterschiedlichen Konfliktpotenzialsituationen zum Konfliktmanagement beizutragen. Die unterschiedlichen Konfliktpotenzialstufen entsprechen den farblich differenzierten Konfliktpotenzialräumen in Abbildung 120. Die Tabelle ist als Entscheidungshilfe für die Maßnahmenauswahl und -priorisierung zu verstehen und sollte im Sinne eines Maßnahmenbaukastens benutzt werden. 
Tabelle 48: Besonders geeignete sektorale Maßnahmenoptionen je Konfliktpotenzialstufe für das Wildschwein.

\begin{tabular}{|c|c|c|c|c|c|c|c|}
\hline \multicolumn{8}{|c|}{$\begin{array}{l}\text { WILDSCHWEIN } \\
\text { Zuordnung von spezifischen Maßnahmenoptionen für Landnutzergruppen zu den Konfliktpotenzialausprägungen }\end{array}$} \\
\hline \multicolumn{2}{|c|}{ Konfliktpotenzialstufe } & 1 & 2 & 3 & $4^{*}$ & 5 & 6 \\
\hline & Nutzungswahrscheinlichkeit (Erholungsnachfrage) & hoch & mittel & hoch & gering & mittel & gering \\
\hline \multicolumn{8}{|c|}{ Maßnahmenoptionen } \\
\hline \multicolumn{8}{|c|}{ Weitere Landnutzergruppen } \\
\hline \multicolumn{8}{|c|}{ Jagdwirtschaft } \\
\hline \multicolumn{2}{|r|}{ Erhöhung der Schwarzwildabschüsse und der Bejagungseffizienz } & $\mathbf{X}$ & $\mathbf{X}$ & $(\mathrm{X})$ & $\mathbf{X}$ & $\mathbf{X}$ & $\mathbf{X}$ \\
\hline \multicolumn{2}{|r|}{ Intensivierte Schwerpunktbejagung in Wildschadensgebieten } & $\mathbf{X}$ & $\mathbf{X}$ & $\mathbf{X}$ & $\mathbf{X}$ & $\mathbf{X}$ & $\mathbf{X}$ \\
\hline \multicolumn{2}{|r|}{$\begin{array}{l}\text { Ausrichtung der Bejagungsstrategie an der Wildschadenanfälligkeit von landwirtschaftlichen } \\
\text { Kulturen }\end{array}$} & $\mathbf{x}$ & $\mathbf{x}$ & $\mathbf{x}$ & $\mathbf{x}$ & $\mathbf{x}$ & $\mathbf{x}$ \\
\hline \multicolumn{2}{|r|}{ Erhaltung störungsberuhigter Einstandsräume des Schwarzwilds } & $\mathbf{x}$ & $\mathbf{x}$ & $(\mathrm{X})$ & $(\mathrm{X})$ & $(\mathrm{X})$ & \\
\hline \multicolumn{2}{|c|}{ Revierübergreifende Bejagungsstrategie } & $\mathbf{x}$ & $\mathbf{X}$ & $\mathbf{x}$ & $\mathbf{X}$ & $\mathbf{X}$ & $\mathbf{X}$ \\
\hline \multicolumn{2}{|r|}{ Vereinbarung definierter Jagdtage bzw. -zeiten mit anderen Landnutzergruppen } & $\mathbf{X}$ & $\mathbf{X}$ & $\mathbf{X}$ & & & \\
\hline \multicolumn{2}{|r|}{ Zeitlich befristete Jagdsperrgebiete } & $\mathbf{X}$ & $\mathbf{X}$ & $\mathbf{X}$ & & & \\
\hline \multicolumn{2}{|c|}{ Abschussvorgaben in Jagdpachtverträgen } & $\mathbf{X}$ & $\mathbf{X}$ & $\mathbf{X}$ & $\mathbf{X}$ & $\mathbf{X}$ & $\mathbf{X}$ \\
\hline \multicolumn{8}{|c|}{ Forstwirtschaft } \\
\hline \multicolumn{2}{|c|}{ Schaffung effizienter Bejagungsmöglichkeiten im Wald } & $\mathbf{X}$ & $\mathbf{X}$ & $\mathbf{x}$ & $\mathbf{x}$ & $\mathbf{X}$ & $\mathbf{x}$ \\
\hline \multicolumn{2}{|r|}{ Forstliche Beiträge zur Vermeidung von Wildschäden an landwirtschaftlichen Kulturen } & $\mathbf{X}$ & $\mathbf{X}$ & $\mathbf{x}$ & $\mathbf{X}$ & $\mathbf{X}$ & $\mathbf{X}$ \\
\hline \multicolumn{8}{|c|}{ Landwirtschaft } \\
\hline \multicolumn{2}{|r|}{$\begin{array}{l}\text { Landwirtschaftliche Maßnahmen zur Verringerung der Wildschadenanfälligkeit von Agrar- } \\
\text { kulturen }\end{array}$} & $\mathbf{X}$ & $\mathbf{x}$ & $\mathbf{x}$ & $x$ & $\mathbf{x}$ & $x$ \\
\hline \multicolumn{2}{|r|}{ Verbesserung der Bejagbarkeit von landwirtschaftlichen Offenlandflächen } & $\mathbf{X}$ & $\mathrm{X}$ & $\mathbf{X}$ & $\mathbf{X}$ & $\mathrm{X}$ & $\mathrm{X}$ \\
\hline \multicolumn{8}{|c|}{ Monitoring und adaptives Management } \\
\hline \multicolumn{8}{|c|}{\begin{tabular}{|l|l|}
$N$ & Erfolgskontrolle zu vereinbarten Nutzungsregeln \\
\end{tabular}} \\
\hline N1 & Monitoring der Wirksamkeit von getroffenen Nutzungsvereinbarungen & $\mathbf{x}$ & $\mathbf{x}$ & $\mathbf{x}$ & & & \\
\hline 0 & Monitoring von Wildtierpopulationen und -lebensräumen & & & & & & \\
\hline
\end{tabular}




\begin{tabular}{|c|c|c|c|c|c|c|c|}
\hline \multicolumn{8}{|c|}{$\begin{array}{l}\text { WILDSCHWEIN } \\
\text { Zuordnung von spezifischen Maßnahmenoptionen für Landnutzergruppen zu den Konfliktpotenzialausprägungen }\end{array}$} \\
\hline \multicolumn{2}{|c|}{ Konfliktpotenzialstufe } & 1 & 2 & 3 & $4^{*}$ & 5 & 6 \\
\hline & Nutzungswahrscheinlichkeit (Erholungsnachfrage) & hoch & mittel & hoch & gering & mittel & gering \\
\hline \multicolumn{8}{|c|}{ Maßnahmenoptionen } \\
\hline 01 & Einrichtung eines regelmäßigen Schwarzstorch-Monitorings & & & & & & \\
\hline $\mathrm{O} 2$ & Fortführung des Ziesel-Monitorings & & & & & & \\
\hline $\mathrm{O} 3$ & Monitoring von Schalenwildeinfluss auf die Waldvegetation & & & & & & \\
\hline $\mathrm{O} 4$ & Monitoring von Wildtierbeständen, Wildabschüssen und Fallwild & $\mathbf{x}$ & $\mathbf{X}$ & $\mathbf{X}$ & $\mathbf{x}$ & $\mathbf{x}$ & $\mathbf{X}$ \\
\hline O5 & $\begin{array}{l}\text { Zusätzliches Monitoring spezifischer Wildtierindikatorarten (Bodenbrüter sowie Ein- } \\
\text { fluss des Schwarzwilds auf deren Bestände) }\end{array}$ & $\mathrm{x}$ & $\mathbf{x}$ & $\mathbf{x}$ & $\mathbf{x}$ & $\mathbf{X}$ & $\mathbf{x}$ \\
\hline O6 & Integration eines Wildtier-Monitorings in das allgemeine Biodiversitäts-Monitoring & & & & & & \\
\hline $\mathrm{O} 7$ & Monitoringpflicht in Natura 2000-Gebieten & & & & & & \\
\hline
\end{tabular}

Zeichenerklärung: $\quad$ X ... prioritäre Managementoption; $(\mathbf{X})$... mit Vorbehalt geeignete bzw. weniger prioritäre Managementoption 


\subsubsection{Auerhuhn}

Die Hinweise und Entscheidungshilfen dieses Kapitels 4.3.2.4 zu den Zielen und zur Maßnahmenauswahl für das Konfliktmanagement beziehen sich ausschließlich auf die Indikatorart Auerhuhn. Es ist darauf hinzuweisen, dass jedes Konfliktmanagement in konkreten Teilräumen des Biosphärenparks stets auch Konfliktpotenziale und Managementbedarf in Bezug auf die anderen Indikatorarten berücksichtigen und auf deren Zusammenschau basieren sollte.

\subsection{Modellierung des Konfliktpotenzials im GIS}

Das Auerhuhn unterscheidet sich grundsätzlich von den übrigen Indikatorarten, weil es aktuell im Biosphärenpark nur vereinzelt vorkommt und keine eigenständige Population bildet. Über die Ausbildung von Metapopulationsstrukturen und ein bestehendes angrenzendes Auerwildvorkommen, das als Quellpopulation fungieren könnte, wäre jedoch theoretisch eine Wiederansiedlung des Auerhuhns im Wienerwald denkbar. Die lebensraumbezogenen Angaben in Bezug auf das Auerhuhn im Biosphärenpark beziehen sich daher nicht auf die aktuelle Verteilung und Lebensraumnutzung, sondern auf das lebensraumbezogene Wiederansiedlungspotenzial. Die in der GIS-Modellierung ausgewiesenen Konfliktpotenziale würden daher erst dann Aussagekraft und Relevanz erlangen, wenn die Re-Etablierung des Auerhuhns als explizites Ziel im Biosphärenpark angestrebt würde. Dies würde ein konkretes Bekenntnis insbesondere auf Seiten der betroffenen Grundeigentümer zu einem auf die Habitatansprüche des Auerhuhns ausgerichteten Waldmanagement erfordern.

Die nachfolgende Kartendarstellung (Abbildung 122) zeigt drei unterschiedliche Ausprägungsformen des Konfliktpotenzials zwischen der Indikatorart Auerhuhn und der Summe aller Indikator-Freizeitaktivitäten im Biosphärenpark Wienerwald. Das Konfliktpotenzial ergibt sich aus der Kombination der summierten Nutzungswahrscheinlichkeit aller Freizeitaktivitäten mit dem Lebensraum- bzw. Wiederansiedlungspotenzial für das Auerhuhn.

Für die Modellierung im GIS wurde die stufenlose Standardisierung der Konfliktpotenziale zwischen 0 und 1, wie sie in den Kartendarstellungen des Kapitels 3.4.3 vorgenommen wurde, auf eine diskrete Skala mit drei Werteklassen des Konfliktpotenzials umgerechnet. Hierfür wurde die Nutzungswahrscheinlichkeit der Indikatoraktivitäten linear in drei Werteabschnitte, d.h. in drei gleich große Skalenintervalle (hoch, mittel, gering), klassifiziert. Betreffend das lebensraumbezogene Wiederansiedlungspotenzial für das Auerhuhn wurde nur eine einzige Klasse verwendet, wobei die Kategorien historische Vorkommen, rezente Sichtungen und potenzielle Lebensraumeignung gemäß der Vorkommenskarte in Kap. 3.4.2.3 (Abbildung 85) als „Wiederansiedlungspotenzial vorhanden“ gewertet wurden. In allen anderen Teilen des Biosphärenparks wurde nach der im Projekt angewendeten Methodik kein relevantes Wiederansiedlungspotenzial ermittelt.

Durch die Kombination von drei Klassen der Nutzungswahrscheinlichkeit mit einer Klasse des Wiederansiedlungspotenzials für das Auerhuhn ergeben sich insgesamt drei Kombinationsmöglichkeiten, die unterschiedlichen Ausprägungsformen des Konfliktpotenzials entsprechen. Jeder der drei Konfliktpotenzialausprägungen wird ein eigener Farbwert zugewiesen. In allen anderen Teilen des Biosphärenparks, wo keine Hinweise auf vorhandenes Lebensraumpotenzial für das Auerhuhn erhoben wurden, besteht auch kein Konfliktpotenzial mit Freizeitaktivitäten; diese konfliktpotenzialfreien Bereiche sind in der Karte weiß dargestellt. Die Kombination erfolgt über die Verknüpfungsmatrix in Abbildung 123 mittels der darin festgelegten Verknüpfungsregeln.

Die Karte in der nachstehenden Abbildung 122 zeigt die Lage und räumliche Verteilung der drei Konfliktpotenzialstufen im Biosphärenpark. In den nachfolgenden Kapiteln werden den unterschiedlichen Konfliktpotenzialausprägungen differenzierte Managementziele sowie prio- 
ritäre Maßnahmenoptionen für das Besuchermanagement und für weitere spezifische Landnutzergruppen zugeordnet. Über die Karte in Abbildung 122 ist näherungsweise die räumliche Zuordnung von vorrangigen Zielen und Maßnahmenoptionen des Konfliktmanagements zu Konflikträumen mit unterschiedlicher Konfliktpotenzialausprägung möglich. 


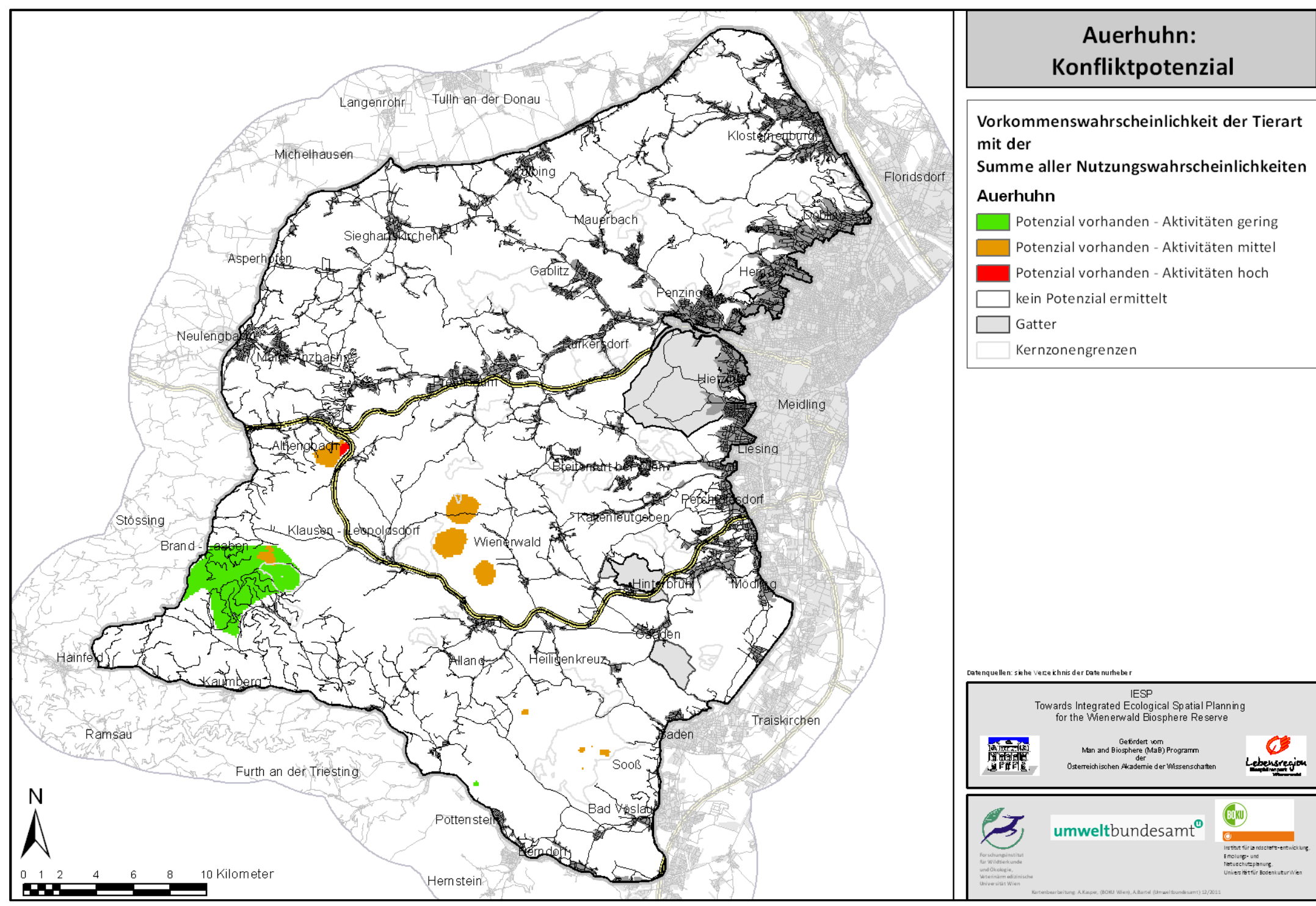

Abbildung 122: Räumliche Verteilung des Konfliktpotenzials zwischen dem Auerhuhn und der Summe aller Freizeitaktivitäten in drei Werteklassen. 


\subsection{Verknüpfungsmatrix}

Mittels nachstehender Abbildung 123 wird das Lebensraumpotenzial für das Auerhuhn mit der summierten Nutzungswahrscheinlichkeit aller Indikator-Freizeitaktivitäten verknüpft. Über die aus der Abbildung ersichtlichen Vernüpfungsregeln ergeben sich drei Kombinationsmöglichkeiten, die unterschiedlichen Ausprägungsformen bzw. Stufen des Konfliktpotenzials entsprechen. Die Verknüpfungsmatrix erleichtert die Zuordnung von Zielen für das Konfliktmanagement (siehe 4.3.2.4.4) sowie von besonders geeigneten Maßnahmenoptionen (siehe Kap. 4.3.2.4.5 und Kap. 4.3.2.4.7) zu unterschiedlichen Konflikt(potenzial)situationen. Über die zugewiesenen Farbwerte wird weiters die näherungsweise räumliche Zuordnung von prioritären Managementoptionen in der Karte in Abbildung 122 (Kap. 4.3.2.4.1) möglich.
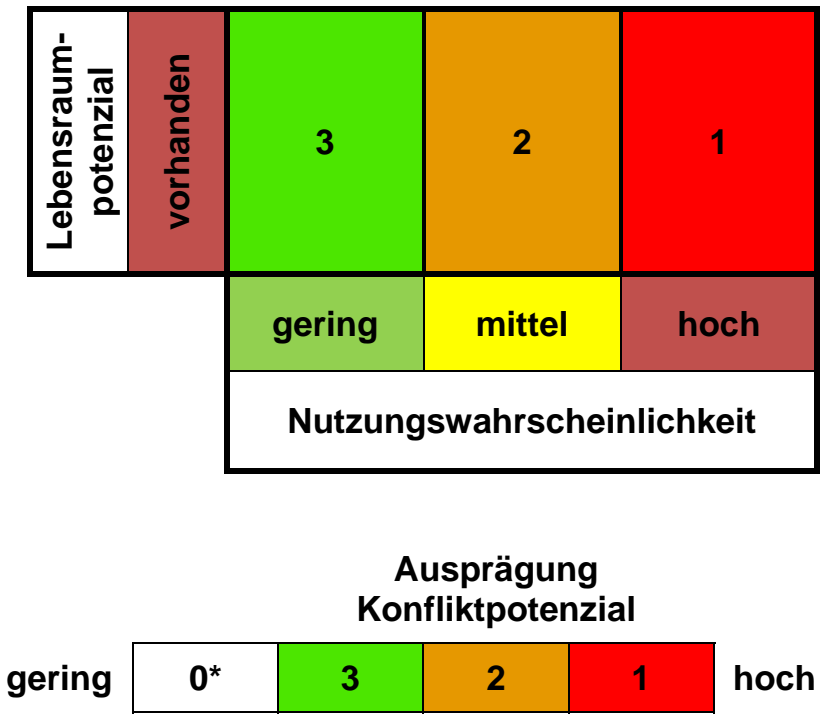

Anmerkung: * 0 ... kein Lebensraumpotenzial ermittelt, daher kein Konfliktpotenzial mit Freizeitaktivitäten.

Abbildung 123: Verknüpfungsmatrix (oben) des Lebensraumpotenzials des Auerhuhns mit der Summe der Nutzungswahrscheinlichkeiten aller Freizeitaktivitäten sowie Farbskala der Ausprägung des Konfliktpotenzials (unten)

\section{Erläuterungen:}

Lebensraumpotenzial: Bezeichnet das Vorhandensein von lebensraumbezogenem Wiederansiedlungspotenzial für das Auerhuhn. Historische Vorkommen, rezente Sichtungen und aktuell geeignete Habitatbedingungen wurden als Indizien für bestehendes Wiederansiedlungspotenzial gewertet. Es werden nur die beiden Fälle „Lebensraumpotenzial vorhanden“ und „kein Lebensraumpotenzial ermittelt“ unterschieden. Unter der Voraussetzung, dass die Wiederansiedlung des Auerhuhns zukünftig gewünscht sein sollte, indiziert vorhandenes Lebensraumpotenzial, dass die betreffende Fläche gegenüber Störungseinflüssen durch die Indikator-Erholungsaktivitäten grundsätzlich hoch sensibel ist.

Nutzungswahrscheinlichkeit: Fungiert als Maß für die modellierten Nutzungsintensitäten aller Indikator-Erholungsaktivitäten. Es werden drei linear skalierte Werteklassen (hoch, mittel, gering) differenzert. Die Nutzungswahrscheinlichkeit ist als potenzielle Erholungsnachfrage auf Basis theoretischer Annahmen und von GIS-basierten Modellierungen zu verstehen. 
Konfliktpotenzialausprägung bzw. Konfliktpotenzialstufe: Aus der Verknüpfung des Lebensraumpotenzials mit der Nutzungswahrscheinlichkeit ergeben sich im Fall des Auerhuhns drei unterschiedliche Konfliktpotenzialausprägungen. Jede Kombination von Vorkommens- und Nutzungswahrscheinlichkeit repräsentiert eine spezifische Konflikt(potenzial)situation, die wiederum jeweils spezifische Managementziele und spezifischen Managementbedarf erfordern kann. Unter "Managementbedarf“ ist zu verstehen, dass auf Flächen unterschiedlicher Ausprägung von Konfliktpotenzialen jeweils unterschiedliche Maßnahmenoptionen in unterschiedlicher Kombination als besonders geeignet erscheinen können, um die vorrangigen Managementziele zu erreichen. Die Verknüpfungsmatrix erleichtert somit einerseits die Zuordnung von prioritären Maßnahmenoptionen zu bestimmten Konfliktsituationen und andererseits über die Farbcodes der Karte in Abbildung 122 die näherungsweise räumliche Zuordnung zu entsprechenden Konflikträumen. 


\subsection{Generelle Ziele für das Konfliktmanagement in Bezug auf das Auerhuhn}

Das Auerhuhn ist eine bodenbrütende Waldvogelart, die den Bestimmungen der Vogelschutzrichtlinie der EU unterliegt. Diese sieht einerseits die Verpflichtung zur Aufrechterhaltung eines positiven Erhaltungszustands der Populationen des Auerhuhns vor, andererseits ist die Bejagung des Auerwilds, als Art des Anhangs II/2 der Richtlinie, in Österreich unter bestimmten Voraussetzungen und Bedingungen nicht ausgeschlossen (Richtlinie 79/409/EWG; Wildauer et al. 2008). Das Auerhuhn unterliegt daher in Österreich als jagdbare Federwildart auch den Bestimmungen des Jagdrechts der Bundesländer. Generelle Ziele in Bezug auf die Indikatorart Auerhuhn ergeben sich somit einerseits aus dem EUNaturschutzrecht und dessen Umsetzung in den österreichischen Landesnaturschutzgesetzen, andererseits auch aus den Landesjagdgesetzen und den zugehörigen Verordnungen. Hinzu kommen potenzielle betriebliche Interessen und Zielsetzungen der Grundeigentümer, für deren Jagdgebiete sich durch eine Wiederansiedlung des Auerhuhns im Biosphärenpark die Möglichkeit einer signifikanten jagdlichen Attraktivitätsteigerung, und damit einer Steigerung des Jagdwertes und des Erlöses aus der jagdlichen Bewirtschaftung, ergeben können.

In jedem Fall setzt eine mögliche Wiederansiedlung des Auerhuhns im Wienerwald ein Bekenntnis der betroffenen Grundeigentümer zum aktiven Habitatmanagement und zum Habitatschutz voraus, was eine Unterordnung forstwirtschaftlicher Zielsetzungen unter das Ziel des Auerwildschutzes erfordern würde. Leitprinzipien im Fall einer gewünschten WiederEtablierung des Auerhuhns sind daher der Schutz und das gezielte Management von Auerwildhabitaten mit vorhandenem Lebensraumpotenzial. Dies schließt konsequente Besucherlenkungsmaßnahmen zur ganzjährigen Störungsberuhigung, die Vermeidung betrieblicher oder anderweitiger landnutzungsbedingter Störungen sowie jagdliche Begleitmaßnahmen zur Regulation von Fressfeinden mit ein.

Unter der Voraussetzung, dass die Wiederansiedlung des Auerhuhns auf Flächen mit vorhandenem Lebensraumpotenzial im Biosphärenpark als Ziel angestrebt werden sollte, sind im Hinblick auf die Steuerung von Konfliktpotenzialen mit Freizeit- und Erholungsnutzungen in der biosphärenparkweiten Betrachtung somit vor allem folgende übergeordnete Ziele maßgeblich:

- Entwicklung, Verbesserung und Schutz geeigneter Auerhuhnhabitate durch gezieltes forstliches bzw. waldbauliches Habitatmanagement und Einrichtung von Habitatschutzgebieten

o In Kernzonenbereichen: Prüfung der Vereinbarkeit von auerwildfreundlichem Waldmanagement mit Zielen des Prozessschutzes (ungestörte Waldentwicklung)

- Ganzjährige Vermeidung und Minimierung von Störungen (Balzzeit, Jungenaufzucht, winterlicher Nahrungs- und Energieengpass) durch Freizeitaktivitäten und andere Beunruhigungsfaktoren

- Jagdliche Bestandeskontrolle der Schwarzwildpopulation und lokale Regulation anderweitiger Fressfeinde zur Sicherung der Auerwildpopulation und des Bruterfolges 


\subsection{Auerhuhn: Zuordnung von Leitfunktionen und Managementzielen zu Kon- fliktpotenzialstufen}

In der nachstehenden Tabelle 49 werden prioritäre Leitfunktionen und Zielausrichtungen für das Konfliktmanagement in Bezug auf die Indikatorart Auerhuhn nach Ausprägungsformen des Konfliktpotenzials differenziert. Die Priorisierungen und Zuweisungen von Leitfunktionen und Managementzielen spiegeln den unterschiedlichen Managementbedarf je nach Konfliktpotenzialstufe wider und sind als Orientierungshilfe für die nachfolgende Auswahl besonders geeigneter Managementoptionen zu verstehen. Zu beachten ist, dass Konfliktpotenziale zwischen den Lebensraumansprüchen des Auerhuhns und Freizeitaktivitäten - und damit ein Bedarf nach Konfliktmanagement - erst unter der Voraussetzung enstehen, dass die Wiederansiedlung des Auerhuhns zu einem Ziel im Biosphärenpark wird.

Tabelle 49: Zuordnung von Leitfunktionen und Managementzielen zu Konfliktpotenzialausprägungen für das Auerhuhn.

\begin{tabular}{|c|c|c|c|c|}
\hline \multicolumn{5}{|c|}{$\begin{array}{l}\text { AUERHUHN: } \\
\text { Zuordnung von Leitfunktionen und Managementzielen zu Konfliktpotenzialausprägungen }\end{array}$} \\
\hline 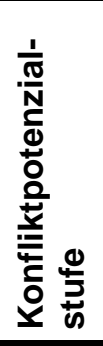 & 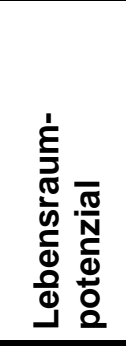 & 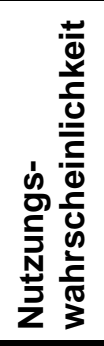 & Leitfunktion & Ziele \\
\hline 1 & $\begin{array}{l}\text { vor- } \\
\text { handen }\end{array}$ & hoch & \multirow{2}{*}{$\begin{array}{l}\text { Habitatschutz } \\
\text { Auerhuhn - } \\
\text { Nutzungs- } \\
\text { beruhigung, } \\
\text { Störungsreduktion }\end{array}$} & \multirow{2}{*}{$\begin{array}{l}\text { - Entwicklung und Verbesserung geeigneter } \\
\text { Auerhuhnhabitate (Balzplätze, Brut- und } \\
\text { Aufzuchtreviere, Sommer- und Wintereinstände, } \\
\text { Nahrungsangebot) durch gezieltes forstliches } \\
\text { Habitatmanagement } \\
\text { - Ganzjährige Reduktion von Störungen infolge von } \\
\text { Freizeitbetrieb und Bewirtschaftungsmaßnahmen; } \\
\text { gezielte Beruhigung von Auerhuhnhabitaten durch } \\
\text { aktive und restriktive Besucherlenkungsmaßnahmen } \\
\text { - Jagdliche Begleitmaßnahmen zur Populationssiche- } \\
\text { rung (Bestandeskontrolle Schwarzwild; lokale Regu- } \\
\text { lation Fressfeinde) } \\
\end{array}$} \\
\hline 2 & $\begin{array}{l}\text { vor- } \\
\text { handen }\end{array}$ & mittel & & \\
\hline 3 & $\begin{array}{l}\text { vor- } \\
\text { handen }\end{array}$ & gering & $\begin{array}{l}\text { Habitatschutz } \\
\text { Auerhuhn - } \\
\text { Erhaltung geringer } \\
\text { Störungsintensität } \\
\text { (Ruhig halten) }\end{array}$ & $\begin{array}{l}\text { - Erhaltung störungsarmer Auerhuhnhabitate durch } \\
\text { ganzjähriges Geringhalten der Erholungsnachfrage; } \\
\text { Vermeiden von Störungen, die zu Verschlechterun- } \\
\text { gen für Habitate und Populationszustand des Auer- } \\
\text { huhns führen können } \\
\text { - Entwicklung und Verbesserung geeigneter } \\
\text { Auerhuhnhabitate (Balzplätze, Brut- und } \\
\text { Aufzuchtreviere, Sommer- und Wintereinstände, } \\
\text { Nahrungsangebot) durch gezieltes forstliches } \\
\text { Habitatmanagement } \\
\text { - Jagdliche Begleitmaßnahmen zur Populationssiche- } \\
\text { rung (Bestandeskontrolle Schwarzwild; lokale Regu- } \\
\text { lation Fressfeinde) }\end{array}$ \\
\hline 0 & \multicolumn{2}{|c|}{$\begin{array}{l}\text { Kein Lebens- } \\
\text { raumpotenzial } \\
\text { ermittelt, daher } \\
\text { kein Konfliktpo- }\end{array}$} & $\begin{array}{l}\text { Kein Auerhuhn- } \\
\text { spezifischer } \\
\text { Managementbedarf }\end{array}$ & $\begin{array}{l}\text { - Derzeit kein besonderer Managementbedarf aus der } \\
\text { Indikatorart Auerhuhn ableitbar } \\
\text { - Vorbehaltsbereich für Ziele und Maßnahmen, die } \\
\text { sich in Bezug auf andere Indikatorarten ergeben }\end{array}$ \\
\hline
\end{tabular}

IESP - Towards Integrated Ecological Spatial Planning for the Wienerwald Biosphere Reserve 


\section{\begin{tabular}{|l||c||c|c|}
\hline & tenzial & & können \\
\hline
\end{tabular} \\ 4.3.2.4.5 Auerhuhn: Übersicht über Handlungsoptionen des Besuchermanagements und querschnittsorientierte Maßnahmenoptionen nach Konfliktpotenzialstu- fen}

In der nachstehenden Tabelle 50 werden die in Kap. 4.2 beschriebenen Maßnahmenoptionen aus den Bereichen Besuchermanagement und querschnittsorientierte Maßnahmen den Konfliktpotenzialstufen in Bezug auf das Auerhuhn zugeordnet. Die Nummerierung der Maßnahmen entspricht der Gliederung in Kap. 4.2. Die Zordnung gibt Hinweise darauf, welche Maßnahmen grundsätzlich besonders geeignet erscheinen, um in unterschiedlichen Konfliktpotenzialsituationen zum Konfliktmanagement beizutragen. Die unterschiedlichen Konfliktpotenzialstufen entsprechen den farblich differenzierten Konfliktpotenzialräumen in Abbildung 122. Die Tabelle ist als Entscheidungshilfe für die Maßnahmenauswahl und -priorisierung zu verstehen und sollte im Sinne eines Maßnahmenbaukastens benutzt werden. 
Tabelle 50: Besonders geeignete Maßnahmenoptionen (Besuchermanagement, Querschnittsmaßnahmen) je Konfliktpotenzialstufe für das Auerhuhn.

\begin{tabular}{|c|c|c|c|c|c|}
\hline \multicolumn{6}{|c|}{$\begin{array}{l}\text { AUERHUHN } \\
\text { Zuordnung von Maßnahmenoptionen (Besuchermanagement, Querschnittsmaßnahmen) zu den Konfliktpotenzialausprägungen }\end{array}$} \\
\hline \multicolumn{2}{|c|}{ Konfliktpotenzialstufe } & 0 & 1 & $\overline{2}$ & 4 \\
\hline & "Nutzungswahrscheinlichkeit (Erholungsnachfrage) & & hoch & mittel & gering \\
\hline \multicolumn{6}{|c|}{ Maßnahmenoptionen } \\
\hline $\mathrm{Nr}^{*}$ & Bezeichnung & & & & \\
\hline \multicolumn{6}{|c|}{ Besuchermanagement - Freizeit- und Erholungsaktivitäten } \\
\hline \multicolumn{6}{|c|}{ Kleinräumige Maßnahmenoptionen } \\
\hline $\boldsymbol{A}$ & Räumlich-zeitliche Nutzungsrestriktionen & & & & \\
\hline A1 & Vereinheitlichung rechtlich verankerter Nutzungsverbote und -gebote (Kernzonen) & $\mathbf{x}$ & $\mathbf{x}$ & $\mathbf{x}$ & $\mathbf{x}$ \\
\hline A2 & Verzicht auf Ausbau der Wegeinfrastruktur & & $\mathbf{X}$ & $\mathbf{X}$ & $\mathbf{X}$ \\
\hline A3 & Verlegung bzw. Auflassung von Wegen und kernzonengerechtes Wegenetz & & $\mathbf{X}$ & $\mathbf{X}$ & $\mathbf{X}$ \\
\hline A4 & $\begin{array}{l}\text { Freiwillige Vereinbarungen zu örtlich begrenzten, räumlichen und/oder zeitlichen Nutzungseinschrän- } \\
\text { kungen }\end{array}$ & & $\mathbf{x}$ & $x$ & $(\mathrm{X})$ \\
\hline$B$ & Besucherlenkung durch De-Attraktivierung & & & & \\
\hline B1 & Landschaftsgestalterische Maßnahmen zur Verringerung der Zugänglichkeit & & $\mathbf{x}$ & $\mathbf{x}$ & $\mathbf{X !}$ \\
\hline B2 & Verzicht auf Schneeräumung & & $\mathbf{X}$ & $\mathbf{X}$ & $\mathrm{X} !$ \\
\hline B3 & Management von Infrastruktur mit Schleusenfunktion & & $\mathbf{X}$ & $\mathbf{X}$ & $(\mathrm{X})$ \\
\hline C & Kleinräumige Kompensations- bzw. Alternativangebote zur Besucherlenkung & & & & \\
\hline $\mathrm{C} 1$ & Hundeauslaufbereiche & $(\mathrm{X})$ & & & \\
\hline $\mathrm{C} 2$ & Night Trails für Jogger & $(\mathrm{X})$ & & & \\
\hline C3 & Night Trails und Winter Trails für Mountainbiker & $(X)$ & & & \\
\hline C4 & Mountainbike Technik Trails & $(X)$ & & & \\
\hline C5 & Mountainbike Downhill-Strecken & $(X)$ & & & \\
\hline C6 & Betreute Picknickplätze & $(\mathrm{X})$ & & & \\
\hline $\mathrm{C} 7$ & Wildlife Viewing-Einrichtungen (Schwarzstorch-Beobachungswarten) & & & & \\
\hline $\mathrm{C} 8$ & Verstärkte Instandhaltung und Pflege der bestehenden Wegeinfrastruktur & $(X)$ & & & \\
\hline
\end{tabular}




\begin{tabular}{|c|c|c|c|c|c|}
\hline \multirow{2}{*}{\multicolumn{2}{|c|}{$\begin{array}{l}\text { AUERHUHN } \\
\text { Zuordnung von Maßnahmenoptionen (Besuchermanagement, Querschnittsmaßnahmen) zu de } \\
\text { Konfliktpotenzialstufe }\end{array}$}} & Kol & enz & $\lg$ & \\
\hline & & 0 & 1 & 2 & 4 \\
\hline Nutzungswahrscheinlichkeit (Erholungsnachfrage) & \begin{tabular}{r|r} 
Lebensraumpotenzial (Sensibilität) \\
Nutzoit (Frholungnachfrage)
\end{tabular} & & hoch & mittel & gering \\
\hline \multicolumn{6}{|c|}{ Maßnahmenoptionen } \\
\hline$D$ & \multicolumn{5}{|l|}{ Verstärkte Information, Kontrolle und Vollzug in Schwerpunktbereichen } \\
\hline D1 & Konsequente Durchsetzung des Wegegebotes in Kernzonen & & $\mathrm{X}$ & $\mathrm{X}$ & $\mathbf{x}$ \\
\hline $\mathrm{D} 2$ & Verstärkte Information, Kontrolle und Sanktionierung in sensiblen Gebieten & & $\mathrm{X}$ & $\mathrm{X}$ & $(\mathrm{X})$ \\
\hline D3 & $\begin{array}{l}\text { Verstärkte Information, Kontrolle und Sanktionierung im Bereich von Kompensations- und Alternativ- } \\
\text { angeboten (siehe C) }\end{array}$ & $(\mathrm{X})$ & & & \\
\hline$E$ & Teilraumbezogene Beurteilung von Zielpriorität und Managementbedarf & \multirow{2}{*}{\multicolumn{4}{|c|}{ nicht relevant }} \\
\hline E1 & $\begin{array}{l}\text { Prüfung des spezifischen Managementbedarfs und situationsabhängige Priorisierung von Handlungs- } \\
\text { strategien und Maßnahmenoptionen (A, B, D) in Bezug auf das Auerhuhn oder andere Indikatorarten }\end{array}$ & & & & \\
\hline $\boldsymbol{F}$ & \multicolumn{5}{|l|}{ Information, Kommunikation, Öffentlichkeitsarbeit } \\
\hline F1 & Zielgruppenspezifische Kommunikation und Öffentlichkeitsarbeit & \multirow{5}{*}{\multicolumn{4}{|c|}{$\begin{array}{l}\text { Geeignete Integration von kohärenten Inhalten in bio- } \\
\text { sphärenparkweite Maßnahmen }\end{array}$}} \\
\hline $\mathrm{F} 1.1$ & Interne Kommunikation & & & & \\
\hline $\mathrm{F} 1.2$ & Aus- und Weiterbildung & & & & \\
\hline $\mathrm{F} 1.3$ & Kommerzielle Angebote & & & & \\
\hline $\mathrm{F} 1.4$ & Kommunikationsschleusen & & & & \\
\hline \multicolumn{2}{|r|}{ - Nutzung von kleinräumigen Attraktivierungsangeboten $(\mathrm{C} 1-\mathrm{C} 6)$ als Kommunikationsschleusen } & $(\mathrm{X})$ & & & \\
\hline F2 & $\begin{array}{l}\text { Biosphärenparkweite und zielgruppenspezifische Konzepte für Kommunikation und Öffentlichkeitsar- } \\
\text { beit }\end{array}$ & \multicolumn{4}{|c|}{$\begin{array}{l}\text { Biosphärenparkweite Anwendung unter Berücksichti- } \\
\text { gung der unterschiedlichen Konfliktpotenziale }\end{array}$} \\
\hline F3 & Schaffung von Kommunikationsstrukturen mit institutionalisierten Vertretern & \multicolumn{4}{|c|}{ Biosphärenparkweite Anwendung } \\
\hline \multirow[t]{3}{*}{ F4 } & Zentrale Internet-Marketingplattform für naturbasierte Freizeitaktivitäten & \multicolumn{4}{|c|}{$\begin{array}{l}\text { Biosphärenparkweite Anwendung unter Berücksichti- } \\
\text { gung der unterschiedlichen Konfliktpotenziale }\end{array}$} \\
\hline & - Weglenkung von Hauptlebensräumen bzw. Wildschutzgebieten & & $\mathrm{x}$ & $\mathrm{X}$ & $\mathrm{X}$ \\
\hline & - Ausweisung von kleinräumigen Attraktivierungsangeboten für Freizeitnutzungen $(\mathrm{C} 1$ - C6) & $(\mathrm{X})$ & & & \\
\hline F5 & Naturraumverträgliches Geocaching-Konzept & \multicolumn{4}{|c|}{$\begin{array}{l}\text { Biosphärenparkweite Anwendung unter Berücksichti- } \\
\text { gung der unterschiedlichen Konfliktpotenziale }\end{array}$} \\
\hline & - Weglenkung von Hauptlebensräumen bzw. Wildschutzgebieten & & $\mathbf{x}$ & $X$ & $x$ \\
\hline
\end{tabular}

IESP - Towards Integrated Ecological Spatial Planning for the Wienerwald Biosphere Reserve 


\begin{tabular}{|c|c|c|c|c|c|}
\hline \multicolumn{6}{|c|}{$\begin{array}{l}\text { AUERHUHN } \\
\text { Zuordnung von Maßnahmenoptionen (Besuchermanagement, Querschnittsmaßnahmen) zu den Konfliktpotenzialausprägungen }\end{array}$} \\
\hline \multicolumn{2}{|c|}{ Konfliktpotenzialstufe } & 0 & $\overline{1}$ & $\overline{2}$ & $\overline{4}$ \\
\hline \multicolumn{2}{|r|}{$\begin{array}{ll} & \text { Lebensraumpotenzial (Sensibilität) } \\
\end{array}$} & & hoch & hoch & hoch \\
\hline \multicolumn{2}{|r|}{ Nutzungswahrscheinlichkeit (Erholungsnachfrage) } & & hoch & mittel & gering \\
\hline \multicolumn{6}{|c|}{ Maßnahmenoptionen } \\
\hline \multirow[t]{3}{*}{ F6 } & Verbesserung der Beschilderung & \multicolumn{4}{|c|}{$\begin{array}{l}\text { Biosphärenparkweite Anwendung unter Berücksichti- } \\
\text { gung der unterschiedlichen Konfliktpotenziale }\end{array}$} \\
\hline & \multirow{2}{*}{$\begin{array}{l}\text { - wirksamere Information über räumlich-zeitliche Nutzungsrestriktionen }(\mathrm{A} 1-\mathrm{A} 5) \\
\text { - unter Verweis auf lokale Kompensationsangebote }(\mathrm{C} 1-\mathrm{C} 6) \text { andernorts }\end{array}$} & & $\mathrm{x}$ & $\mathrm{X}$ & $(\mathrm{X})$ \\
\hline & & & $\mathbf{x}$ & $\mathbf{X}$ & $(\mathrm{X})$ \\
\hline \multirow[t]{3}{*}{$\mathrm{F} 7$} & Digitale Geoinformationen für Wegeverwaltung und Routenplanung & \multicolumn{4}{|c|}{$\begin{array}{l}\text { Biosphärenparkweite Anwendung unter Berücksichti- } \\
\quad \text { gung der unterschiedlichen Konfliktpotenziale }\end{array}$} \\
\hline & - Weglenkung von Hauptlebensräumen bzw. Wildschutzgebieten & & $\mathrm{x}$ & $\mathrm{X}$ & $\mathrm{x}$ \\
\hline & - Ausweisung von kleinräumigen Attraktivierungsangeboten für Freizeitnutzungen (C1 - C6) & $(\mathrm{X})$ & & & \\
\hline F9 & Schulung von Aufsichts- und Kontrollorganen & \multirow{2}{*}{\multicolumn{4}{|c|}{$\begin{array}{l}\text { Biosphärenparkweite Anwendung unter Berücksichti- } \\
\text { gung der unterschiedlichen Konfliktpotenziale }\end{array}$}} \\
\hline \multicolumn{2}{|r|}{ - Kommunikations- und Konfliktmanagement } & & & & \\
\hline F10 & Fortbildungsangebot & \multicolumn{4}{|c|}{$\begin{array}{l}\text { Geeignete Integration von relevanten Inhalten in Fort- } \\
\text { bildungs- und Kursangebote für Freizeitnutzergruppen }\end{array}$} \\
\hline F11 & Positive Kommunikation von Restriktionen über (Kompensations-)Angebote & \multicolumn{4}{|c|}{$\begin{array}{l}\text { Biosphärenparkweite Anwendung in allen relevanten } \\
\text { Kommunikationsmaßnahmen }\end{array}$} \\
\hline F12 & Verzicht auf Maßnahmen zur Bewerbung und zum Freizeitmarketing & & $\mathbf{x}$ & $\mathbf{X}$ & $\mathbf{x}$ \\
\hline G & Kooperative Konfliktlösungsstrategien & & & & \\
\hline \multicolumn{6}{|c|}{ G1 - G4 (Details siehe Kap. 4.1.3.2.2) } \\
\hline \multicolumn{2}{|r|}{$\begin{array}{l}\text { - zur Aushandlung freiwilliger Vereinbarungen zu örtlich begrenzten, räumlichen und/oder zeitlichen } \\
\text { Nutzungsrestriktionen für den Freizeitbetrieb }(A 1-A 5)\end{array}$} & & $\mathbf{X}$ & $\mathbf{x}$ & $(\mathrm{X})$ \\
\hline \multicolumn{2}{|r|}{$\begin{array}{l}\text { - zur Standortfindung und Entwicklung kleinräumiger Kompensations- bzw. Alternativangebote für } \\
\text { Freizeitaktivitäten }(C 1-C 7)\end{array}$} & $(\mathrm{X})$ & & & \\
\hline \multicolumn{2}{|r|}{$\begin{array}{l}\text { - zur Vereinbarung von Maßnahmen, die verstärkte sektorübergreifende Koordination zwischen } \\
\text { Landnutzergruppen erfordern }\end{array}$} & & $\mathbf{X}$ & $\mathbf{X}$ & $\mathbf{X}$ \\
\hline$H$ & Kontrolle und Vollzug von Verhaltensregeln & \multirow{2}{*}{\multicolumn{4}{|c|}{$\begin{array}{l}\text { Biosphärenparkweite Anwendung unabhängig von } \\
\text { Konfliktpotenzialausprägung }\end{array}$}} \\
\hline \multicolumn{2}{|c|}{ H1 - H2 (Details siehe Kap. 4.1.3.2.2) } & & & & \\
\hline
\end{tabular}




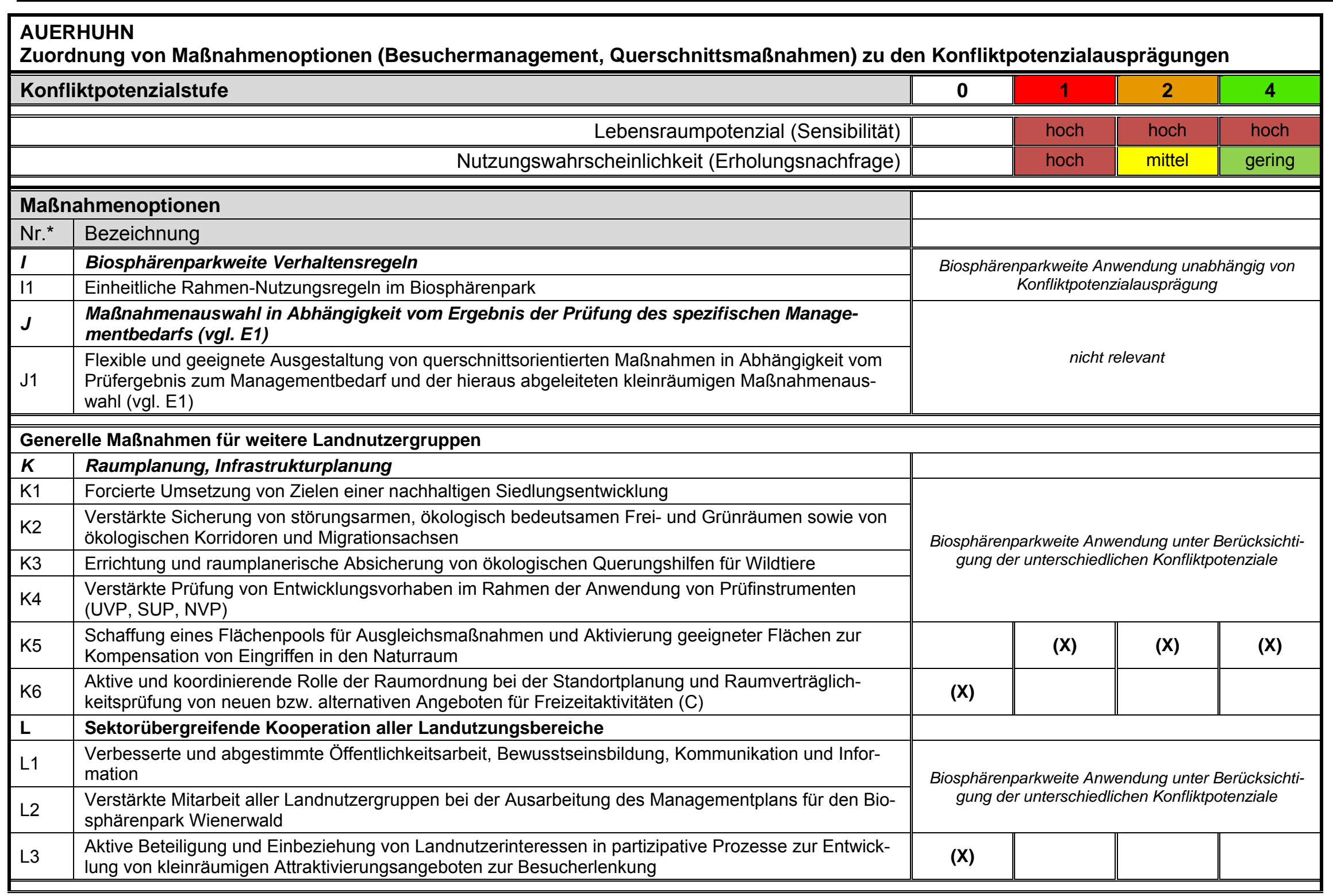




\begin{tabular}{|c|c|c|c|c|c|}
\hline \multicolumn{6}{|c|}{$\begin{array}{l}\text { AUERHUHN } \\
\text { Zuordnung von Maßnahmenoptionen (Besuchermanagement, Querschnittsmaßnahmen) zu den Konfliktpotenzialausprägungen }\end{array}$} \\
\hline \multicolumn{2}{|c|}{ Konfliktpotenzialstufe } & 0 & 1 & $\overline{2}$ & $\overline{4}$ \\
\hline & Lebensraumpotenzial (Sensibilität) & & hoch & hoch & hoch \\
\hline & Nutzungswahrscheinlichkeit (Erholungsnachfrage) & & hoch & mittel & gering \\
\hline \multicolumn{6}{|c|}{ Maßnahmenoptionen } \\
\hline $\mathrm{Nr}^{*}$ & Bezeichnung & & & & \\
\hline \multicolumn{6}{|c|}{ Monitoring und adaptives Management } \\
\hline$M$ & Besuchermonitoring & & & & \\
\hline M1 & Besuchermonitoring mit Hilfe anonymisierter Bewegungsdaten von HandynutzerInnen & (X) & $\mathbf{x}$ & $\mathbf{X}$ & $(\mathrm{X})$ \\
\hline M2 & Beobachtungen und Zählungen von Besucherströmen & (X) & $\mathbf{x}$ & $\mathbf{x}$ & $\mathbf{x}$ \\
\hline M3 & Befragungen & \multicolumn{4}{|c|}{$\begin{array}{l}\text { Durchführung wird biosphärenparkweit empfohlen; } \\
\text { Befragungsdesign und Auswertung sollten unter- } \\
\text { schiedliche Konfliktpotenziale und entsprechende Ma- } \\
\text { nagementoptionen berücksichtigen }\end{array}$} \\
\hline$N$ & Erfolgskontrolle zu vereinbarten Nutzungsregeln & & & & \\
\hline N1 & Monitoring der Wirksamkeit von getroffenen Nutzungsvereinbarungen & (X) & $\mathbf{X}$ & $\mathbf{x}$ & $\mathbf{X}$ \\
\hline
\end{tabular}

Zeichenerklärung: $\quad X$... prioritäre Managementoption; $(X) \ldots$ mit Vorbehalt geeignete bzW. weniger prioritäre Managementoption; $X !$... stark prioritäre Managementoption 


\subsection{Spezifische Maßnahmenoptionen für weitere Landnutzergruppen in Bezug auf das Auerhuhn}

Die nachfolgend beschriebenen Maßnahmenoptionen haben zur Voraussetzung, dass die Etablierung einer überlebensfähigen Auerhuhnpopulation im Biosphärenpark als Ziel angestrebt wird.

\section{Naturschutz}

Erstellung eines naturschutzfachlichen und wildökologischen Konzepts zur Etablierung und Erhaltung einer überlebensfähigen Auerhuhnpopulation auf regionaler Ebene: Die Flächen mit vorhandenem Lebensraumpotenzial für das Auerhuhn im Wienerwald stellen kleinflächige und mosaikartig zersplitterte Habitatinseln dar. Die Etablierung einer überlebensfähigen Auerhuhnpopulation im Wienerwald ist nur im Rahmen von Metapopulationsstrukturen und durch die populationsökologische Konnektivität mit bestehenden Quellpopulationen außerhalb des Biosphärenparks, d.h. durch Einwanderung, möglich. Die Voraussetzung dafür, dass natürliche Zuwanderung stattfinden kann, ist die Schaffung geeigneter Lebensraumbedingungen in Potenzialgebieten innerhalb des Biosphärenparks. Die Entwicklung von Auerhuhnhabitaten erfordert einerseits örtliche Managementpläne, die von den betroffenen Forstbetrieben gemeinsam mit Artenschutzexperten bzw. Wildökologien erstellt werden sollten. Andererseits scheint aufgrund der beschriebenen Abhängigkeit des Auerhuhns von Metapopulationsstrukturen ein regionsweites Konzept zur Etablierung und Erhaltung überlebensfähiger Populationen des Auerhuhns empfehlenswert, das Quellpopulationen und Auerhuhnkerngebiete außerhalb des Biosphärenparks sowie Anforderungen an die ökologische Konnektivität berücksichtigt. Dem Naturschutz käme bei der Erarbeitung eines derartigen Konzeptes unter anderem eine wichtige koordinierende Rolle zu.

Abstimmung von Anforderungen des Auerwildschutzes mit den Managementzielen in relevanten Kernzonen: Einige der in diesem Projekt ermittelten Gebiete mit Lebensraumpotenzial für das Auerhuhn liegen innerhalb von Kernzonen des Biosphärenparks bzw. haben Anteil an solchen. In den Kernzonen des Biosphärenparks gilt das Ziel des Prozessschutzes, d.h. der ungestörten Entwicklung von Waldökosystemen; forstliche Nutzungseingriffe sind in diesem Sinne in Kernzonen untersagt. Die Zielsetzung des Prozessschutzes kann zu den Lebensraumansprüchen des Auerhuhns im Widerspruch stehen. Die Schaffung und dauerhafte Erhaltung von Habitatbedingungen, wie sie das Auerhuhn benötigt, erfordert im Wienerwald oftmals gezieltes waldbauliches Habitatmanagement, einschließlich gestaltender Eingriffe in Baumartenmischungen und Bestandesstrukturen. Sollte die Wiederansiedlung des Auerhuhns im Wienerwald zukünftig tatsächlich ein Ziel des Biosphärenparks werden, dann kann es notwendig werden, für einzelne betroffene Kernzonen nach einem Ausgleich für den Zielkonflikt zwischen aktivem Habitatmanagement und dynamischem Prozess-Naturschutz zu suchen. Zumindest kleinflächig sollte in diesem Zusammenhang eine flexiblere Handhabung von KernzonenManagementzielen erwogen werden. In jedem Fall wären Aspekte des Auerwildschutzes in die betreffenden Kernzonen-Managementpläne zu integrieren.

\section{Forstwirtschaft}

Forstliches und waldbauliches Habitatmanagement zur Entwicklung geeigneter Auerhuhnhabitate: Als bodenbrütende Waldvogelart hat das Auerhuhn sehr charakteristische Lebensraumansprüche. Bevorzugte Lebensräume sind zusammenhängende, lichte 
Mischwälder mit geringem Kronenschlussgrad, überwiegendem Nadelholzanteil (Fichte, Lärche), offener bzw. mosaikartiger Waldstruktur und einem hohen Anteil an beerentragenden Sträuchern im Unterwuchs bei gleichzeitig fehlender Vergrasung. Häufig repräsentieren lichte Altholzbestände aus Kahlschlagwirtschaft geeignete Habitate für das Auerwild. Der Lebensraum sollte vielfältig strukturiert sein, um den spezifischen Ansprüchen des Auerwilds an geeignete Balzplätze, Brut- und Aufzuchtreviere, Sommer- und Wintereinstände zu entsprechen. Ein hoher Anteil von Grenzlinien und das gemeinsame Vorhandensein von Deckung und Äsung sind günstig. Vorratsreiche, dicht geschlossene Bestände ohne entsprechende Bodenvegetation bieten dem Auerhuhn keine geeigneten Lebensbedingungen

Angesichts der vorherrschenden Waldstruktur im Wienerwald sind gezielte forstliche Eingriffe und waldbauliche Habitatgestaltungsmaßnahmen zur Entwicklung geeigneter Lebensraumbedingungen für das Auerhuhn unvermeidlich. Eine zentrale Voraussetzung wäre daher das konkrete Bekenntnis der Forstbetriebe bzw. Waldeigentümer zu einem auf die Bedürfnisse des Auerhuhns ausgerichteten Waldmanagement. Dies erfordert in den betreffenden, potenziell habitatgeeigneten Waldbeständen die Unterordnung sonstiger forstbetrieblicher Zielsetzungen unter den Auerwildschutz.

Zur Entwicklung und Erhaltung geeigneter Auerhuhnhabitate sollte ein forstlicher bzw. waldbaulicher Habitatmanagementplan auf forstbetrieblicher Ebene erstellt werden, der in enger Zusammenarbeit mit Artenschutzexperten bzw. Wildökologen erarbeitet werden sollte. Da es sich bei den Flächen mit vorhandenem Lebensraumpotenzial im Wienerwald um Habitatinseln handelt, und die Etablierung einer überlebensfähigen Auerhuhnpopulation nur im Rahmen von Metapopulationsstrukturen in Verbindung mit bestehenden Quellpopulationen außerhalb des Biosphärenparks möglich ist, sollten lokale Managementpläne idealerweise in ein regionsweites Auerhuhn-Erhaltungskonzept eingebettet sein.

Vermeidung von Störungen durch forstbetriebliche Tätigkeiten: Das Auerhuhn ist ein störungssensible und europaweit gefährdete Art mit sehr spezifischen Habitatansprüchen in unterschiedlichen Phasen des Lebens- und Reproduktionszyklus. Erhöhte Störungssensibilität besteht grundsätzlich in allen Jahreszeiten, insbesondere zur Zeit der Vorbalz und des Balzgeschehens im Frühjahr, zur eigentlichen Brutzeit und zur Zeit der Jungenaufzucht im (Früh)Sommer sowie zur Zeit des winterlichen Nahrungs- und Energieengpasses. Störungen brütender Hennen, die diese zum Verlassen des Geleges zwingen, können zum Ausfall des Bruterfolges führen. Störungen im Winter können aufgrund empfindlicher Konditionseinbussen die Mortalitätsrate in populationsgefährdendem Ausmaß erhöhen, insbesondere im Zusammenspiel mit widrigen Witterungsbedingungen und Prädatoren. Störungen während der sensiblen Reproduktionsphasen, die den Erhaltungszustand von Populationen beeinträchtigen können, sind auch aufgrund von Vorgaben der Vogelschutzrichtlinie der EU zu vermeiden; die gesamte Brutperiode des Auerhuhns im Sinne der Richtlinie dauert von Anfang April bis Mitte Juli.

Hieraus ergibt sich, dass Störungen durch forstbetriebliche Tätigkeiten, wie Holzernte oder Pflegeeingriffe, möglichst ganzjährig minimiert bzw. kritische Zeiten erhöhter Störungssensibilität (wie Brutperiode und Winter) bei der Arbeitsplanung unbedingt berücksichtigt werden sollten. Die zeitliche und räumliche Planung von Waldarbeiten und eingriffen in diesem Sinne sollte ein Bestandteil des forstlichen Managementplans sein.

\section{Jagdwirtschaft}


Wirksame Bestandeskontrolle des Schwarzwildes und lokale Regulation weiterer Fressfeinde des Auerhuhns: Die flächig hohen und zunehmenden Schwarzwildpopulationen sind derzeit ein limitierender Faktor für eine Wiederansiedlung des Auerhuhns, weil Wildschweine als Prädatoren von Gelegen und Küken des Auerhuhns empfindliche Populationsverluste verursachen können. Eine flächige jagdliche Absenkung des Wildschweinbestandes, die aus landeskulturellen Gründen ohnehin geboten erscheint, wäre demnach eine notwendige Rahmenbedingung für eine erfolgreiche Wiederansiedlung des Auerhuhns. In zukünftigen Auerhuhnhabitaten kann es darüber hinaus erforderlich werden, Schwerpunktbejagungen von Schwarzwild zu intensivieren, wobei jedoch auf die erhöhte Störungssensibilität während der Brutperiode und der Jungenaufzuchtzeit Rücksicht zu nehmen ist.

Zudem kann zur Sicherung von Auerhuhnbeständen die lokale jagdliche Regulation von weiteren Raubfeinden des Auerwilds, insbesondere von kleineren Raubsäugern, als Begleitmaßnahme angezeigt sein. Ein lokales Kurzhalten von Prädatoren darf jedoch keinesfalls mit einem großflächigen „Totalabschuss“ von Raubwild verwechselt werden.

Einrichtung von Wildschutzgebieten gemäß Jagdrecht zum Auerhuhnschutz: Das Niederösterreichische Jagdgesetz (NÖ Jagdgesetz 1974 idgF.) sieht die Möglichkeit der befristeten oder unbefristeten Einrichtung von Wildschutzgebieten vor (§ 94a). Als Wildschutzgebiete dürfen Flächen bewilligt werden, die als besonders bevorzugte Einstandsgebiete Ruhezonen für das Wild sind oder die zum Brüten und Setzen bevorzugt angenommen werden. Die Erklärung zum Wildschutzgebiet bewirkt, dass jagdfremde Personen das betreffende Gebiet abseits von öffenlichen Wegen und Straßen nicht betreten dürfen. Dieses jagdrechtliche Instrument kann somit grundsätzlich in begründeten Fällen zur Verhinderung von Störungen und Beunruhigungen, insbesondere durch Freizeitaktivitäten, eingesetzt werden. Im Hinblick auf potenzielle zukünftige Auerhuhn-Kernhabitate im Biosphärenpark sollte geprüft werden, ob die Einrichtung von Wildschutzgebieten ein zulässiges und geeignetes Instrument zur Erhaltung störungsberuhigter Auerhuhn-Habitate sein kann. Ein vorangehender kooperativer Dialog mit betroffenen Naturraumnutzern sowie begleitende Informations- und Öffentlichkeitsarbeitsmaßnahmen sind in jedem Fall zu empfehlen.

Strenge Reglementierung einer jagdlichen Nutzung des Auerwilds im Einklang mit naturschutzrechtlichen Vorgaben: Das Auerwild ist gemäß der Vogelschutzrichtlinie der EU (Richtlinie 79/409/EWG) eine Vogelart, deren Bejagung in Österreich grundsätzlich und unter bestimmten Voraussetzungen (vgl. Wildauer et al., 2008) zulässig ist. Gemäß dem Konzept der nachhaltigen Nutzung der Biodiversität (UNEP CBD, 1992; UNEP CBD/COP/7/12 2004) kann unter definierten Voraussetzungen die Nutzung einer wildlebenden Ressource durchaus zu ihrer Erhaltung beitragen. Es wird ausdrücklich darauf hingewiesen, dass eine solche Option im Biosphärenpark Wienerwald - wenn überhaupt - noch in ferner Zukunft liegt. In jedem Fall wären im Hinblick auf eine zukünftige Bejagung des Auerwilds die Vorgaben und Einschränkungen der Vogelschutzrichtlinie und des Niederösterreichischen Naturschutzrechts zu beachten. Dies betrifft insbesondere das Verbot der Bejagung während der Brutperiode sowie das Verbot einer Verschlechterung des Erhaltungszustandes von Populationen. Weitere Entscheidungshilfen und Kriterien sind z. B. dem „Leitfaden zu den Jagdbestimmungen der Richtlinie 79/409/EWG des Rates über die Erhaltung der wild lebenden Vogelarten“" (Europäische Kommission 2008) sowie Wildauer et al. (2008) zu entnehmen.

Priorität für Bestandesbegründung durch natürliche Wiedereinwanderung: Das gezielte Aussetzen von Individuen zur Bestandesbegründung ist grundsätzlich aus wildökologischer und jagdpolitischer Sicht als hoch problematisch zu werten. Aus derzeitiger 
Sicht wäre das Einbringen von Auerhühnern auch wirkungslos, weil die Habitatbedingungen gegenwärtig nicht ausreichend sind. Die Entwicklung geeigneter Lebensräume und die Bestandesbegründung durch natürliche Zuwanderung aus umliegenden Quellpopulationen sollte daher in jedem Fall Priorität vor künstlichen Bestandesbegründungen haben. Erst wenn diese Strategie Erfolge zeigt, können Aussetzungen zur Bestandesstützung allenfalls in Erwägung in gezogen werden.

\section{Raumplanung, Infrastrukturplanung}

Sicherung von bestehenden Lebensräumen potenzieller AuerhuhnQuellpopulationen außerhalb des Biosphärenparks: Der Erfolg einer Wiederansiedlung des Auerhuhns im Wienerwald hängt neben der Entwicklung geeigneter Habitate innerhalb des Biosphärenparks in gleichem Maße vom Vorhandensein bestehender Auerwild-Kerngebiete außerhalb des Biosphärenparks ab, die als Quellpopulation für die natürliche Wiedereinwanderung und für die Etablierung funktionierender Metapopulationsstrukturen fungieren können. Ein derartiges Kerngebiet, das als Ausgangspunkt für eine natürliche Wiederbesiedlungs dienen könnte, liegt im Süden des Biosphärenparks. Die im Projekt ermittelten Bereiche mit Lebensraumpotenzial im Biosphärenpark (historische Vorkommen, rezente Einzelnachweise, potenzielle Habitate) liegen grundsätzlich innerhalb von Distanzen zueinander bzw. vom bestehenden Kerngebiet, die durch das Auerhuhn bewältigbar sind.

Um die Option einer Wiederbesiedlung des Biosphärenparks zu bewahren, gilt es daher vorrangig, den bestehenden Auerhuhn-Lebensraum außerhalb der Grenzen des Biosphärenparks zu sichern und die ökologische Konnektivität zu potenziellen Habitatinseln innerhalb des Biosphärenparks zu erhalten. Hierbei ist die Raumordnung gefordert, Baulandwidmungen und Siedlungstätigkeiten, die zu Habitatverlusten bzw. zunehmenden Störungen im Bereich von bestehenden Kernhabitaten führen könnten, konsequent zu vermeiden.

\section{Alle Nutzergruppen}

Betreffend die aktive Beteiligung aller Landnutzergruppen an partizipativen Prozessen zur Entwicklung von kleinräumigen Kompensations- bzw. Alternativangeboten für Freizeitnutzergruppen (vgl. Kap. 4.2.2, Empfehlung L3) siehe die Übersichtsmatrix in Tabelle 50 (Kap. 4.3.2.4.5). 


\subsection{Auerhuhn: Übersicht über spezifische Maßnahmenoptionen für weitere Landnutzergruppen nach Konfliktpotenzialstufen}

In der nachstehenden Tabelle 51 werden die im vorangehenden Kap. 4.3.2.4.6 beschriebenen Maßnahmenoptionen für einzelne Landnutzergruppen sowie die in Kap. 4.2.3 beschriebenen Maßnahmen zum Monitoring von Wildtierpopulationen und -lebensräumen den Konfliktpotenzialstufen in Bezug auf das Auerhuhn zugeordnet. Die Zordnung gibt Hinweise darauf, welche Maßnahmen grundsätzlich besonders geeignet erscheinen, um in unterschiedlichen Konfliktpotenzialsituationen zum Konfliktmanagement beizutragen. Die unterschiedlichen Konfliktpotenzialstufen entsprechen den farblich differenzierten Konfliktpotenzialräumen in Abbildung 122. Die Tabelle ist als Entscheidungshilfe für die Maßnahmenauswahl und -priorisierung zu verstehen und sollte im Sinne eines Maßnahmenbaukastens benutzt werden. 
Tabelle 51: Besonders geeignete sektorale Maßnahmenoptionen je Konfliktpotenzialstufe für das Auerhuhn.

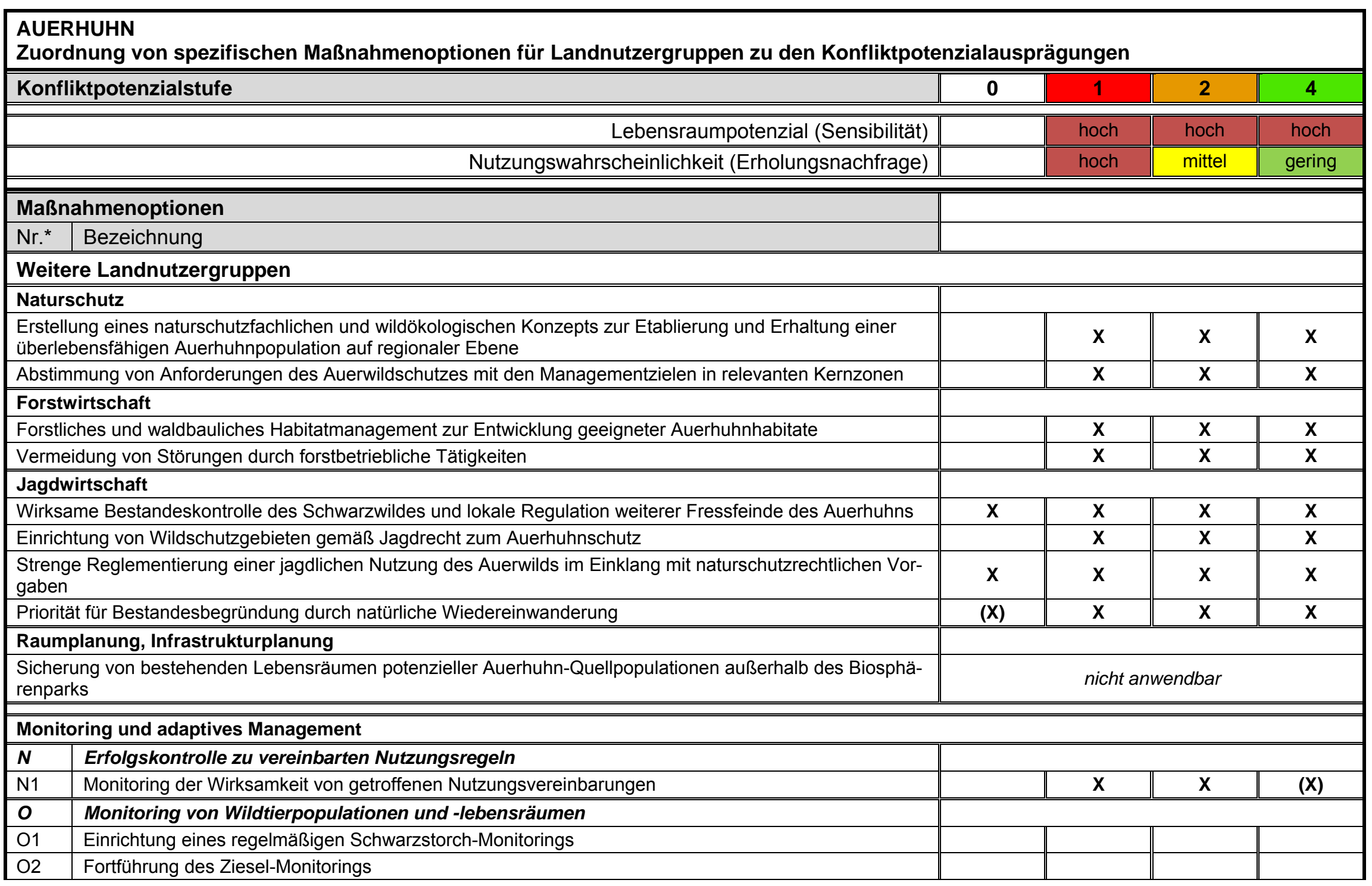




\begin{tabular}{|c|c|c|c|c|c|}
\hline \multicolumn{6}{|c|}{$\begin{array}{l}\text { AUERHUHN } \\
\text { Zuordnung von spezifischen Maßnahmenoptionen für Landnutzergruppen zu den Konfliktpotenzialausprägungen }\end{array}$} \\
\hline \multicolumn{2}{|c|}{ Konfliktpotenzialstufe } & 0 & 1 & $\overline{2}$ & 4 \\
\hline & Nutzungswahrscheinlichkeit (Erholungsnachfrage) & & hoch & mittel & gering \\
\hline \multicolumn{6}{|c|}{ Maßnahmenoptionen } \\
\hline ב3 & Monitoring von Schalenwildeinfluss auf die Waldvegetation & & & & \\
\hline $\mathrm{O} 4$ & Monitoring von Wildtierbeständen, Wildabschüssen und Fallwild & & $\mathbf{X}$ & $\mathbf{X}$ & $\mathbf{X}$ \\
\hline O5 & $\begin{array}{l}\text { Zusätzliches Monitoring spezifischer Wildtierindikatorarten (Bodenbrüter sowie Einfluss des Schwarz- } \\
\text { wilds auf deren Bestände) }\end{array}$ & & $\mathrm{x}$ & $\mathrm{x}$ & $\mathrm{x}$ \\
\hline $\mathrm{O} 6$ & Integration eines Wildtier-Monitorings in das allgemeine Biodiversitäts-Monitoring & & $\mathbf{X}$ & $\mathbf{X}$ & $\mathbf{X}$ \\
\hline O7 & Monitoringpflicht in Natura 2000-Gebieten & & $\mathbf{x}$ & $\mathbf{X}$ & $\mathbf{x}$ \\
\hline O8 & Monitoring der Wiesen, inkl. Wildschadensituation & & & & \\
\hline
\end{tabular}

Zeichenerklärung: $\quad$ X ... prioritäre Managementoption; $(\mathbf{X})$... mit Vorbehalt geeignete bzw. weniger prioritäre Managementoption 


\subsection{Konfliktaustragung und -behandlung auf der Perchtoldsdorfer Heide - ein Good Practice-Beispiel für den Wienerwald}

Bei den Konfliktrecherchen in den Intensivuntersuchungsgebieten ist deutlich geworden, dass viele Konflikte im Bereich Wildtiermanagement - Freizeitnutzung bislang entweder als Konfliktpotenzial oder als latenter Konflikt bestehen. Eine unmittelbare Konfliktaustragung und -bewältigung erfolgt gegenwärtig nicht. Die Ursachen hierfür können unterschiedlich sein. Sie reichen von einem zu geringen Konfliktpotenzial, welches noch nicht zu einer Konfliktaustragung geführt hat, bis zu resignativen Komponenten, wenn eine Konfliktaustragung derzeit nicht als erfolgversprechend angesehen wird. Auf der Perchtoldsdorfer Heide (siehe auch Kap. 3.3.3) sind die entstehenden Konflikte nicht nur aktiv ausgetragen, sondern auch durch eine Kombination von verschiedenen Maßnahmen eine Konfliktbewältigung in Angriff genommen worden. Die sich seit den 1990er Jahren verstärkende Konfliktsituation konnte damit deutlich entschärft und die Konfliktpotenziale konnten reduziert werden.

Das Beispiel Perchtoldsdorfer Heide zeigt zudem erneut, wie eng die beiden Konflikttypen (vgl. Kapitel 2.6.5) verknüpft sind und wie wichtig es ist, dass die Konfliktmanagementmaßnahmen ineinander greifen. Das räumliche Konfliktpotenzial besteht darin, dass eine intensive Freizeitnutzung mit Picknicken, Mountainbiking, Joggen, Aktivitäten mit Hund usw. in einem sehr sensiblen Lebensraum stattfindet. Gefährdet erscheinen dadurch zunächst die Ziesel, die hier eines der wenigen Vorkommen im Osten Österreichs haben, aber natürlich auch eine Vielzahl anderer Tierarten. Die Nutzungsansprüche und das raum-zeitliche Verhalten bei den verschiedenen Freizeitaktivitäten unterscheiden sich deutlich. So erfolgt etwa das Picknicken auf der Fläche und vor allem im Sommer, während Wandern, Joggen und Mountainbiking weggebundene Aktivitäten darstellen, die zu allen Jahreszeiten ausgeübt werden, wenngleich mit unterschiedlicher Intensität. Konfliktverschärfend kommt hinzu, dass die eigentlich linienhafte Freizeitaktivität Mountainbiking durch Fahrten quer über die Heide flächenhaft wirkt und auch unangeleinte Hunde auf der Heide unterwegs sind. Dabei handelt es sich überwiegend um Verstöße gegen vorhandene Regelungen und Verbote.

Die entstandene Konfliktsituation - nicht nur in Bezug auf die Wildtiere im Lebensraum Perchtoldsdorfer Heide, sondern auch zwischen den Freizeitnutzergruppen - hatte sich bis 2003/04 derartig verschärft. dass eine Lösung unmittelbar notwendig wurde. Durch die räumliche Konzentration verschiedener Freizeitaktivitäten und unterschiedlicher Konflikte in einem relativ kleinen Gebiet kann die Heide als eine komplexe Konfliktsituation beschrieben werden. Mehrere Konflikte stehen in einem engen Zusammenhang und sind durch ein Bündel von Maßnahmen zu bearbeiten. Eine Differenzierung der einzelnen Konfliktpotenziale und Konflikte, wie sie in der Diskussion mit den Experten vor Ort vorgenommen wurde, hat eher eine konfliktanalytische Funktion. In der folgenden Darstellung der Konfliktmanagementstrategien wird daher vor allem auf Zusammenhänge und Wirkungsbeziehungen eingegangen.

\section{Konfliktpotenziale und Konfliktgegenstand}

Die zentralen Konflikte auf der Perchtoldsdorfer Heide betreffen die Auseinandersetzung mit Personen, die ihren Hund bzw. ihre Hunde frei laufen lassen sowie mit Mountainbikern, die quer über die Heide fahren. Hinzu kam ein wachsendes Müllproblem nach Silvesterfeiern. Das Anfüttern von Tieren und das Sammeln von Kräutern werden dagegen bislang als Einzelprobleme angesehen.

Die Konfliktträchtigkeit freilaufender Hunde besteht nicht nur darin, dass sie eine erhebliche Störwirkung verursachen oder sogar Wildtiere jagen, sie stellen auch für andere Freizeitnutzer wie Familien mit Kindern eine potenzielle Gefahr oder zumindest Verunsicherung dar. Zudem trägt der Hundekot zur Veränderung der Heidevegetation durch Überdüngung bei, und er kann die Gesundheit der Besucher, z.B. spielender Kinder, gefährden oder zu einer 
Erkrankung der Schafe führen. Die Argumentation der Hundebesitzer ist dann häufig, der Hund brauche Freilauf. Die Reaktion bei einer persönlichen Ansprache ist sehr unterschiedlich und reicht von "gar nicht anhören“ und „nicht mit sich reden lassen“ bis zu Verständnis.

Das Mountainbiking ist auf der Heidestraße in der Sommersaison von Mai bis Oktober tagsüber erlaubt. Es gibt allerdings keine ausgewiesene Mountainbike-Route entlang der Heidestraße mehr, da die Gemeinde sich am Verein nicht mehr beteiligt. Das Problem liegt einerseits darin, dass Mountainbiker auf der Heidestraße zu schnell unterwegs sind und durch ihre wenig rücksichtsvolle Fahrweise insbesondere die zu Fuß gehenden Freizeitnutzer gefährden, und andererseits in Fahrten quer über die Heide. Die Begründungen reichen hierbei von „ich fahre, wo ich will“, „auf der Forststraße ist es fad“ bis zu „am Weg sind zu viele Fußgänger". Der Konflikt beinhaltet somit sowohl Verstöße gegen Regelungen und Nutzungsrestriktionen durch einzelne Mountainbiker als auch die Art und Weise, wie diese Freizeitaktivität ausgeübt wird (Stichwort „Fair Play“-Regeln).

Das Müllproblem zu Silvester hat sich entwickelt, seit zur Jahrtausendwende Plätze für Silvesterfeiern beworben wurden. Den Höhepunkt bildete im Jahr 2007 die Entsorgung von 50 Müllsäcken (vgl. Homepage Perchtoldsdorfer Heide).

Für die Ziesel selbst stellt die Freizeitnutzung an sich ein geringeres Problem dar, mit Ausnahme der freilaufenden Hunde. Sie profitieren sogar davon, dass durch die intensive Freizeitnutzung tagsüber Katzen und auch Greifvögel ferngehalten werden. Durch die Einzäunung eines Zieselschutzgebietes (2004) im Norden der Großen Heide ist der Kernbereich der Zieselkolonie noch besser geschützt. Als problematisch erwies sich aber eine Zeit lang das Anfüttern von Krähen oder Füchsen durch Einzelpersonen, weil diese dann Ziesel und andere Kleintiere gejagt haben.

\section{Konfliktparteien}

Mit der Gründung des Vereins Freunde der Perchtoldsdorfer Heide im Jahr 2000 ist zugleich ein neuer Akteur in die Konfliktaustragung auf der Heide eingetreten, der eine entscheidende Rolle bei der Konfliktbewältigung spielt. Seine Aufgabe sieht der Verein in der Erhaltung und dem Schutz der Perchtoldsdorfer Heide. Das bezieht sich zunächst auf wissenschaftlich fundierte Pflegemaßnahmen, die eine Verbuschung der Trockenrasenareale verhindern sollen. Im Rahmen der jährlich stattfindenden Heidepflegewoche erhält der Verein dabei eine umfangreiche Unterstützung von Vereinen und durch die Bevölkerung. Bei der Heidepflegewoche 2011 haben insgesamt 484 Personen teiilgenommen und 1650 Arbeitsstunden geleistet (Homepage der Perchtoldsdorfer Heide). Darüber hinaus leistet der Verein eine breite Informations- und Aufklärungsarbeit, beispielsweise durch regelmäßige Heideführungen und spezielle Kinderführungen, Informationsbroschüren und Folder sowie die Homepage www.perchtoldsdorfer-heide.at. Für seine zahlreichen Aktionen und Projekte in der Heidepflege und Öffentlichkeitsarbeit (z. B. Jahr der Heide 2004, Heidepflegewoche, Smaragdeidechsenschutzprojekt, GEO-Tag der Artenvielfalt 2007, Erneuerung des Lehrpfads) hat der Verein Freunde der Perchtoldsdorfer Heide bereits einige Auszeichnungen erhalten. Durch die Naturschutzarbeit auf der Heide ist der Verein auch in die Konflikte mit der Freizeitnutzung involviert. Dabei vertritt er aber durchaus auch die Interessen der Erholungsuchunden, wenn es etwa darum geht, die Regeln für Hunde und Mountainbiker um- und durchzusetzen.

Die wichtigsten Regeln für den Erhalt der Heide sind auf der Homepage des Vereins zur Perchtoldsdorfer Heide zusammengefasst:

"- Hunde an die Leine

- Hundekot Ihres Hundes aufsammeln

- Mountainbiken nur auf der dafür freigegebenen Straße 
- Ziesel und andere Tiere bitte nicht füttern

- Keine Kräuter sammeln, Blumen pflücken oder Tiere fangen

- Kein offenes Feuer

- Müll bitte in den Mistkübeln entsorgen“

(Homepage Perchtoldsdorfer Heide: Heide: Erhaltung).

Die Marktgemeinde Perchtoldsdorf ist der Grundeigentümer der Perchtoldsdorfer Heide. In die Belange der Heide sind verschiedene Akteure eingebunden, insbesondere der Bürgermeister, die Gemeindeverwaltung, der Gemeinderat für Land- und Forstwirtschaft und die Polizei. So ist die Leinenpflicht für Hunde auf der gesamten Perchtoldsdorfer Heide (Große und Kleine Heide) durch einen Beschluss des Gemeinderates und eine Ortspolizeiliche Verordnung festgelegt worden (vgl. Leinenzwang auf der Perchtoldsdorfer Heide, Homepage der Gemeinde Perchtoldsdorf). Das Gebiet ist auf einer Karte genau abgegrenzt. Zwischen dem Verein Freunde der Perchtoldsdorfer Heide und der Gemeinde gibt es eine Zusammenarbeit, beispielsweise wird die Arbeit des Vereins mit Sachkosten unterstützt und bei Anfragen zur Durchführung von Veranstaltungen auf der Heide wird der Verein befragt.

Die Kontrolle der Regeln und Nutzungsrestriktionen auf der Perchtoldsdorfer Heide erfolgt durch die Berg- und Naturwacht Mödling, die Flurschutzorgane der Marktgemeinde Perchtoldsdorf und die Polizei. Wenngleich diese vor allem aufklärend wirken, wird bei wiederholter Missachtung auch Anzeige erstattet.

Auf Seiten der Freizeitnutzer bestehen kaum organisatorischen Strukturen, die in Konflikten als Verhandlungspartner agieren könnten. Das trifft auf die Mountainbiker ebenso wie auf die Hundebesitzer zu. In Konflikten aufgrund von Verstößen gegen Verbote und Regelungen werden die Auseinandersetzungen daher in der Regel mit Einzelpersonen oder kleinen Gruppen geführt. Bei den freilaufenden Hunden fällt jedoch eine Kerngruppe von täglichen Besuchern mit Hund auf. Um Auseinandersetzungen mit Einzelpersonen handelt es sich auch beim Füttern von Krähen oder Füchsen.

\section{Konfliktmanagementstrategien}

Als zentraler Akteur bei der Bewältigung der komplexen Konfliktsituation auf der Heide ist der Vereine Freunde der Perchtoldsdorfer Heide aktiv. Seine vielfältigen Aktivitäten haben die Perchtoldsdorfer Heide und ihren Schutz stärker ins Bewusstsein der Bevölkerung und der Besucher gerückt und durch die Einbindung zahlreicher Unterstützer wichtige Multiplikationseffekte erreicht. Dies bildet die Grundlage für konkrete Maßnahmen zur Konfliktbewältigung. Die Hauptstrategie ist auch hierbei die Information.

Aufgrund der zunehmenden Probleme mit freilaufenden Hunden wurde in einer Art Doppelstrategie einerseits auf der gesamten Heide eine Leinenpflicht (2005) eingeführt und andererseits eine große Hundeauslaufzone (2006) zwischen Kröpfgraben (verlängerte Walzengasse) und Kriegsherrgasse eingerichtet. Damit wurden die Restriktionen für Aktivitäten mit Hund im Interesse des Naturschutzes und der anderen Besucher deutlich erhöht, gleichzeitig aber ein attraktives Angebot für das Freilaufenlassen von Hundes geschaffen, das als Treffpunkt für Hundebesitzer zugleich eine soziale Funktion hat. Begleitet wurde beides durch eine umfangreiche Informations- und Aufklärungsarbeit, beispielsweise durch eine entsprechende Beschilderung, einen Folder „Heide-Leitfaden für Frauerl und Herrl“, persönliche Gespräche auf der Heide sowie Informationen auf der Homepage Perchtoldsdorfer Heide. Dabei wird insbesondere erklärt, worin die Gefährdung durch freilaufende Hunde und Hundekot für die Heidevegetation und die Tiere - Wildtiere wie auch weidende Schafe - besteht. Für den Hundekot stehen an den Zugängen und auf der Heide Mistkübelbehälter mit Sackerlspendern zur Verfügung. 
Der Leinenzwang auf der Heide hatte eine gewisse Abwanderung von Personen, die ihren Hund ausführen, zur Folge. Die Hundeauslaufzone wird aber sehr gut angenommen und vor allem in den Morgen- und Abendstunden intensiv genutzt. Es gibt viele positive Rückmeldungen. Die Situation bezüglich Hundekot konnte deutlich verbessert werden. Unterstützend wirkte hier auch die Hundekot-Kampagne der Stadt Wien. Die Situation bei der Leinenpflicht ist noch nicht befriedigend. Dieser Konflikt besteht nach wie vor. Es fehlt in der Gemeinde unter anderem an Kapazitäten, die Informationsarbeit und die Kontrollen auf der Heide zu verstärken. Hier würde sich der Verein eine weitere Unterstützung, etwa von der Gemeinde oder den Weinbauern wünschen, um die Kräfte zu bündeln.

Das Mountainbiking ist nur auf der Heidestraße erlaubt. Um die Fahrten quer über die Heide zu reduzieren, werden wiederum einerseits regelmäßig Kontrollen durchgeführt und andererseits eine umfassende Aufklärung betrieben, insbesondere betreffend Schäden am Habitat durch das off-road fahren. Gleichzeitig versucht der Heideverein auf das rücksichtslose Verhalten eines Teils der Mountainbiker, auch im Interesse der anderen Freizeitnutzer, einzuwirken. Das betrifft vor allem das zu schnelle Fahren auf der Heidestraße. Die Problematik dieses Konflikts besteht wie in anderen Gebieten vor allem darin, dass insbesondere abwärts fahrende Mountainbiker kaum zu stoppen und damit auch nur schwer zu erfassen sind. Gegenüber einzelnen „Wiederholungstätern“ reichen die Kontrollen und Sanktionen sicher noch nicht aus. Der Vorteil auf der Perchtoldsdorfer Heide liegt dennoch darin, dass es mit dem Heideverein einen Akteur gibt, der gemeinsam mit der Gemeinde als Grundeigentümer eine kontinuierliche Information und Aufklärung betreibt. Dies zahlt sich mittelfristig aus. So wird die Situation des Mountainbikings als bereits viel besser eingeschätzt.

Das Müllproblem nach den Silvesterfeiern war Neujahr 2007 so stark eskaliert, dass es dringender Maßnahmen bedurfte. Kernstück war neben der Beseitigung des Mülls durch freiwillige Helfer eine umfassende Aufklärungskampagne, die seither jedes Jahr wiederholt wird. Diese schließt neben der Müllproblematik auch die Störung der Wildtiere durch Raketen und Knaller ein. Aus heutiger Sicht kann eine positive Bilanz aus der intensiven Öffentlichkeitsarbeit der Gemeinde, des Heidevereins und des Liesinger Bezirksvorsteher gezogen werden, denn Neujahr 2011 wurde nur noch ein Sechstel der Müllmenge von 2007 auf der Heide eingesammelt (Homepage Perchtoldsdorfer Heide: Bericht 2011).

Durch die Präsenz des Vereins Freunde der Perchtoldsdorfer Heide und seine Funktion als Ansprechpartner für den Schutz der Heide lassen sich auch eine Reihe weiterer Probleme und Konflikte leichter lösen, wie etwa das Füttern der Ziesel und das Anfüttern von potenziellen Jägern wie Krähen oder Füchsen. Darüber hinaus zeigen sich Multiplikatoreffekte, wenn Spaziergänger andere Freizeitnutzer bei Verstößen auf die Regeln hinweisen oder den Verein informieren, Hundebesitzer andere Hundebesitzer aufklären oder in Mountainbikeforen auf Verbote hingewiesen wird. Wenngleich ein Teil der Konflikte auf der Perchtoldsdorfer Heide noch nicht gelöst ist, sind die positiven Wirkungen des Konfliktmanagements deutlich erkennbar - für die Heideflächen, die Erholungsuchenden, die Mehrheit der Hundebesitzer und die Bevölkerung. 